\title{
Applications of Terrestrial LiDAR for Reducing Uncertainty in Forest Carbon Mapping:
}

\author{
A Scale-Driven Approach
}

Atticus Eythan LeMay Stovall

Floyd, Virginia

B.A. in Environmental Sciences, University of Virginia, 2012

A Dissertation presented to the Graduate Faculty

of the University of Virginia in Candidacy for the Degree of:

Doctor of Philosophy

Department of Environmental Sciences

University of Virginia

November $29^{\text {th }}, 2017$ 


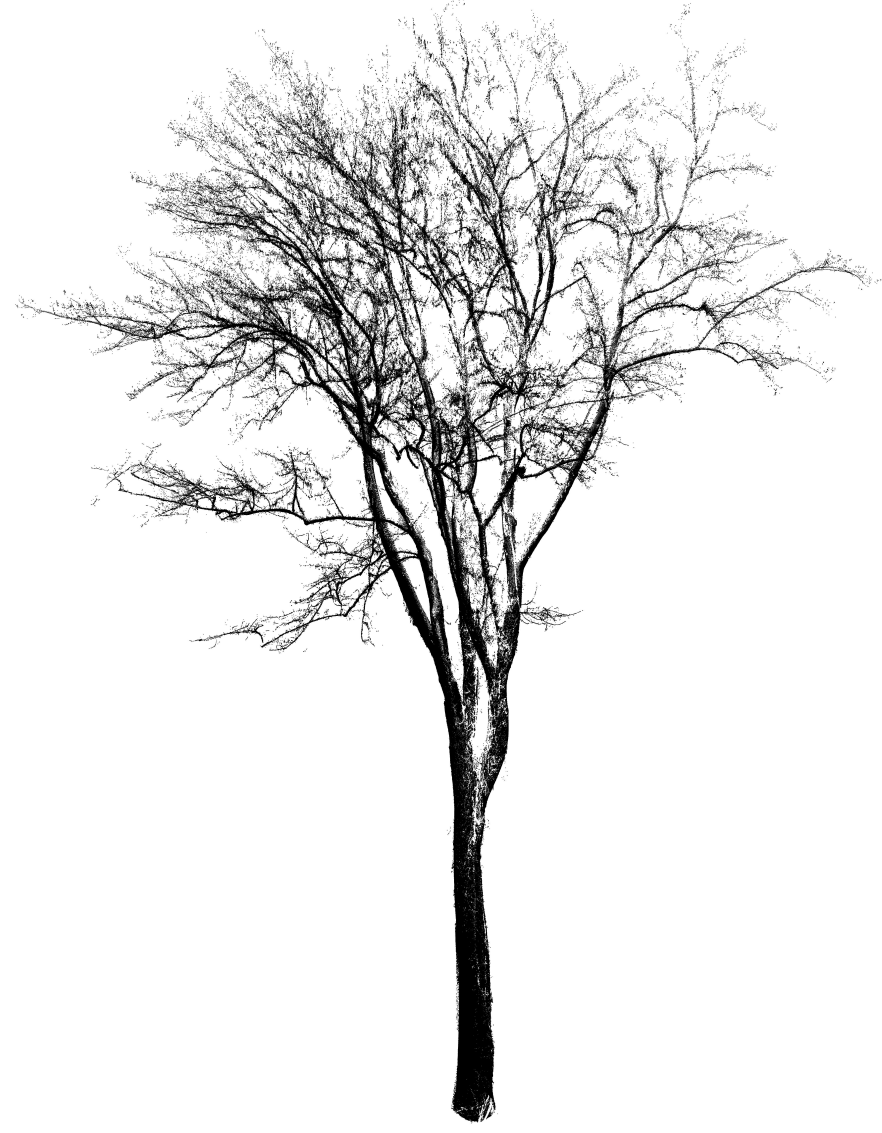

(c) Copyright by Atticus E. L. Stovall 2017

All Rights Reserved 


\begin{abstract}
Forest biomass accounts for the vast majority of aboveground terrestrial carbon storage, but biomass magnitude and spatial distribution is highly uncertain. Global biomass estimates are inextricably linked to tree-level estimates of biomass by way of allometry - an indirect relationship relating tree stem diameter and/or height to destructively harvested dry weight. The difficulty and cost of creating allometric equations has led to spatially biased, low-certainty relationships that limit confidence in global carbon estimates. Efficient, non-destructive biomass estimation with terrestrial laser scanning (TLS) or terrestrial LiDAR can potentially improve single-tree, plot-level, and global biomass estimates through threedimensional modeling, but little is known of how this approach impacts uncertainty at these spatial scales. With a scale-driven analysis, this work explores the potential for TLS to reduce uncertainty in biomass estimates from tree to landscape. At the single-tree scale, a novel algorithm - the Outer Hull Model (OHM) was developed and validated with 21 destructively harvested Pinus contorta trees in the Colorado State Forest. The OHM accurately estimated component (e.g. trunk, branch, foliage) and whole-tree biomass, outperforming other approaches. In a broadleaf deciduous Virginia forest, TLS was used to model over 300 trees for developing species-specific allometry and quantifying errors in commonly used national allometry. TLS-derived non-destructive allometry had lower uncertainty than the national equations. A allometric sample-size-based sensitivity analysis was conducted with and without trees above $50 \mathrm{~cm}$ diameter, revealing a strong dependency on large trees for accurate biomass prediction with allometry. The dependency of airborne and spaceborne LiDAR biomass estimates on plot-level calibration provided an avenue for landscape-scale improvements in uncertainty with TLS. TLS-based LiDAR calibration was compared to traditional methods with three different models based on mean canopy height and return intensity. TLS reduced uncertainty primarily by accurate direct estimates of standing biomass, improvements in allometric uncertainty, and reduced RMSE LiDAR calibration. The scale-driven approach of this work emphasizes the need for improved allometry in forest ecosystems, especially when high quality calibration and validation data is needed - a goal that can be realized with the strategic deployment of TLS in high uncertainty environments.
\end{abstract}




\section{Acknowledgements}

A dissertation cannot be completed without the support and advise of others. This work is no exception and would not be in it's current form without extensive contributions from several individuals.

The destructive harvesting data collected for Chapter 2 was a massive undertaking, and the crew responsible provided an invaluable resource for TLS validation. My collaboration with Tony Vorster, Ryan Anderson and Paul Evangelista has given context and applicability to the non-destructive biomass estimation algorithm. Many thanks to Ryan for collecting and completing initial post-processing on the TLS data and to Tony for processing of the destructive samples and superb editing skills. Paul, for facilitating this collaboration and sponsoring a stellar visit to CO.

The stem map data used in Chapters 3 and 4 was an immense effort from several dedicated students and scientists at SCBI. I am grateful for the hours contributed to these data. Thanks to Kristina Texiera for her graciously providing access to the SIGEO forest plot. Thank to the SIGEO PIs, Norman Bourg and William McShea, for managing and creating the SCBI stem map. Several students and colleagues helped me collect TLS data. In particular, Bella Reyes contributed many hours and always did so with an upbeat attitude. Adrianna Foster was beyond helpful with this field work, taking time away from her dissertation and enriching my work with her time and personal support.

Several colleagues reviewed and edited manuscripts, improving them greatly. Thanks to Jeff Atkins, Tony Vorster, Xi Yang, Lola Fatoyinbo, and Laura Duncanson for contributing along these lines. 
This dissertation work was generously funded by the University of Virginia, NASA, the Virginia Space Grant Consortium, SilvaCarbon, the National Fish and Wildlife Foundation, and Jay Zieman.

Thanks to my committee and advisers for guiding me and helping me understand the context of my work with respect to the broader scientific community. As instructors, Todd and Howie provided much of the foundational knowledge for my dissertation work throughout undergraduate and graduate school. Robert Washington-Allen was an essential resource early on and helped acquaint me with TLS technology.

Hank - I am forever grateful to you for believing in me as a scientist and supporting me though the years. Without your support many of the opportunities I have experienced would not have been possible. You have kindly given me a more global perspective on forest ecosystems that will impact me for many years to come.

To my family and close friends, thank you for your unwavering encouragement.

Adrienne - you have been there for me in life and in the field, giving more than I could have asked. Your loving support made this possible. To my boys, Moses and Asa, I look forward to many more years of walking in the woods with you and all of the new things we will learn together. 


\section{Contents}

Contents vii

List of Figures $\quad$ xiii

List of Tables $\quad$ Xv

1 Forest Structure and Understanding Global Carbon 1

1.1 The Role of Uncertainty in Understanding Forest Structure at Multiple Spatial Scales . . . . . . . . . . . . . . . 2

1.2 Forests in the Global Context . . . . . . . . . . . . . . . 3

1.3 Traditional Methods of Biomass Estimation and Limitations . . . . 6

1.4 Remote Sensing for Biomass Estimation . . . . . . . . . . . . 8

1.4.1 Passive Optical Remote Sensing . . . . . . . . . . . . . 8

1.4.2 Radar Remote Sensing . . . . . . . . . . . . . . . . . 9

1.4.3 LiDAR Remote Sensing: Technological Overview and Biomass Mapping . . . . . . . . . . . . . . . . . . 10

1.5 Terrestrial LiDAR in Forestry . . . . . . . . . . . . . . . 12

1.5.1 Traditional Forestry Measurements with TLS . . . . . . . . 13

1.5.2 Vegetation Structure: Gap Fraction and Voxel Approaches . 15

1.5.3 Biomass and Volume Retrieval: Voxel Approaches . . . . . . 17

1.5.4 Biomass and Volume Retrieval: Primitive-fitting Approaches 18

1.6 General Knowledge Gap and Main Questions . . . . . . . . . . . . . 20

1.7 Dissertation Structure and Study Motivation . . . . . . . . . . . 21

2 Non-Destructive Biomass Estimation from Terrestrial LiDAR 38

2.1 Introduction . . . . . . . . . . . . . . . . . . . . 40

2.2 Methods . . . . . . . . . . . . . . . . . . . 42

2.2.1 Study Area . . . . . . . . . . . . . . . . . . . 42

2.2.2 Destructive Sampling . . . . . . . . . . . . . . . . . . . . . . . . . . . . . . . 46

2.2 .3 Terrestrial LiDAR . . . . . . . . . . . . . . . 46

2.3 Results . . . . . . . . . . . . . . . . . . . . 58

2.3.1 Diameter and Height . . . . . . . . . . . . . . . . . 58

2.3.2 Parameter Optimization . . . . . . . . . . . . 58 
2.3.3 Validation of Non-destructive Biomass Estimates . . . . . . 59

2.4 Discussion . . . . . . . . . . . . . . . . . . . 60

2.4.1 Basic Forestry Measurements . . . . . . . . . . . . . . 62

2.4.2 Non-destructive Biomass Estimation . . . . . . . . . . . 64

2.5 Conclusion . . . . . . . . . . . . . . . . . . . . 68

2.6 Appendix . . . . . . . . . . . . . . . . . . . . . . . . 69

3 Implications of Applying Non-Destructive Local Allometry $\quad 88$

3.1 Introduction . . . . . . . . . . . . . . . . 90

3.2 Methods . . . . . . . . . . . . . . . . . . . 92

3.2.1 Site Location and Dominant Species Selection . . . . . . . . 92

3.2.2 Terrestrial LiDAR Aquisition and Processing . . . . . . . . . . 94

3.2.3 Allometric Development and Analysis . . . . . . . . . . . . . 97

3.3 Results . . . . . . . . . . . . . . . . . . 100

3.3 .1 Allometry . . . . . . . . . . . . . . . . . . 100

3.3.2 Sample Size Analysis . . . . . . . . . . . . . . . . . . . . 103

3.3.3 Comparison to National Equations . . . . . . . . . . . . . 104

3.4 Discussion . . . . . . . . . . . . . . . . . . . . . . . . . . . 108

3.4 .1 Allometry . . . . . . . . . . . . . . . . . . . 109

3.4.2 Sample Size Analysis . . . . . . . . . . . . . . . . . . 111

3.4.3 National Equation Comparison and Evaluation . . . . . . . 112

3.5 Conclusion . . . . . . . . . . . . . . . . . . . . 114

3.6 Appendix . . . . . . . . . . . . . . . . . . 115

4 Improved Biomass Calibration with Terrestrial LiDAR 121

4.1 Introduction . . . . . . . . . . . . . . . . . . . . 123

4.2 Methods . . . . . . . . . . . . . . . . . 125

4.2.1 Study Area and Species Selection . . . . . . . . . . . . 125

4.2.2 TLS Allometry . . . . . . . . . . . . . . . . . . . . 127

4.2.3 LiDAR Biomass Calibration and Validation . . . . . . . . 131

4.3 Results . . . . . . . . . . . . . . . . . . . . . . 134

4.3 .1 TLS Allometry . . . . . . . . . . . . . . . . 134

4.3.2 Biomass Estimation and Uncertainty Analysis . . . . . . . . 135

4.4 Discussion . . . . . . . . . . . . . . . . . . . . . . . . . . . . 138

4.4.1 TLS for Local Non-destructive Allometry . . . . . . . . . . . 138

4.4.2 Reducing Uncertainty in LiDAR Models . . . . . . . . . . . 140

4.5 Conclusion . . . . . . . . . . . . . . . . . . . . . . . 142

5 Moving Forward with Terrestrial LiDAR 149

$\begin{array}{lr}\text { Supplementary Images } & 164\end{array}$ 


\section{List of Figures}

2.1 Flowchart detailing the method developed in this study to estimate tree biomass from terrestrial LiDAR scans. . . . . . . . . . . . . 47

2.2 Visualization of (a) all the tree point clouds used in this study and (b) an example of a point cloud that was (c) segmented into the branch and needle component and trunk component. (d) Volume of the branch and needle component was estimated using voxelization. (e) The Outer Hull Model estimated trunk volume. Each component is multiplied by density to obtain biomass estimates. (f) These components are summed to estimate whole-tree biomass. . . . . .

2.3 Flowchart detailing the method of crown branch and foliage biomass estimation. The $[\mathrm{A}]$ crown returns under a $[\mathrm{B}]$ full coverage scenario are expected to be proportional to total volume, but $[\mathrm{C}]$ occlusion reduced the point coverage in the inner crown. The $[\mathrm{D}]$ voxel density is not representative of branch volume in the presence of significant occlusion effects. [E] The observed mean horizontal voxel density distribution is corrected using the height-specific trunk volume. $[\mathrm{F}]$ Trunk radius is used with $[\mathrm{G}]$ WBE allometric scaling theory to estimate the approximate radius of an inner crown branch $\left(r_{\text {branch }}\right)$ and then innermost mean voxel density $\left(\beta_{1}\right)$. Voxel density represents the percent of filled space in a $2 \mathrm{~cm}$ cube and can be directly converted to an estimate of branch volume. The average innermost crown voxel density is used in a $[\mathrm{H}]$ logarithmic model spanning the horizontal distance from the bole to the crown edge. [I] The corrected distribution is modeled from the observed and modeled distributions, correcting crown voxel density for occlusion effects. 
2.4 Flowchart detailing the method of trunk biomass estimation. [A] The TLS data is separated to obtain the [B] trunk point cloud and the modeling process is initiated. The algorithm begins from the lowest point cloud height and, moving upward, finds the first $[\mathrm{C}]$ trunk subsection height $\left(z_{i}\right)$ with three or more points within a specified thickness. [C] A circle is fit to the subsection points and, if the error in fit exceeds a threshold, the convex hull is computed and points are removed that fall along this initial outer hull. [D] The point removal process continues iteratively $\left(p_{1}, p_{2} \ldots p_{n}\right)$, "peeling" the outermost points of the set until the percent of returns removed reaches a threshold $\left(O H M_{p}\right)$ defined by the calibration process. Occluded portions of the trunk sections $\left(z_{i}\right)$ are partially filled using a $[\mathrm{E}]$ vertical buffer $\left(D_{b}\right)$ that includes trunk points from above $\left(z_{i+D_{b}}\right)$ and below $\left(z_{i-D_{b}}\right)$ for use in the convex hull peeling procedure. The algorithm proceeds until all trunk subsections are modeled $[\mathrm{F}] \ldots \ldots \ldots \ldots \ldots \ldots \ldots \ldots \ldots \ldots \ldots \ldots \ldots \ldots \ldots$

2.5 Comparison of field measured and TLS-based estimates of (a) diameter at breast height and (b) height for living (black) and dead (hollow circles) trees. . . . . . . . . . . . . . . . .

2.6 Parameter optimization process used in the convex hull peeling algorithm. Biomass error was compared to the primary input parameters for each volume estimation approach. Red lines approximate the effect of varying input parameters on biomass error for the single tree used for calibration. The final column (right) shows the final optimized model based on the training subsample used to automate parameter selection. . . . . . . . . . . . . . .

2.7 Non-destructive (a) whole-tree and (b) woody aboveground biomass estimates with terrestrial LiDAR compared to destructively sampled reference biomass for living (black) and dead (open-circles) trees. . .

2.8 Comparison of destructively-sampled and non-destructive TLS-based estimates of (a) trunk, (b) branches, (c) needles, and (d) branches with needles for living (black) and dead (open-circles) trees. . . . .

2.9 Comparison of destructively sampled and non-destructive TLS-based estimates of trunk section biomass $(\mathrm{n}=232)$. Black circles are live tree samples and open circle are dead tree samples. . . . . . . . .

2.10 Example of noise removal procedure. The raw point cloud (top) is denoised with stay point removal and statistical outlier removal. Noise points (middle, in red) are removed for a clean point cloud (bottom). . . . . . . . . . . . . . . . 
2.11 Comparison of [A] trunk point cloud slice and [B] trunk point cloud slice with vertical buffer. Prominent gaps in the lower right of the point cloud due to occlusion are filled using this method. . . . . . .

2.12 Comparison of $[\mathrm{A}]$ least squares circle fit to [B] convex hull peeling as implemented in the OHM algorithm. The reduction in RMSE $(\mathrm{m})$ is substantial on this low noise trunk slice. Points are slightly transparent in order to show locations of high return density (darker). 73

2.13 Comparison of $[\mathrm{A}]$ least squares circle fit to [B] convex hull peeling as implemented in the OHM algorithm. This example includes the voxelization procedure for reduced computation time. Note: RMSE $(\mathrm{m})$ is higher when the point cloud has been voxelized. Points are slightly transparent in order to show locations of high return density (darker). . . . . . . . . . . . . . . . . . . 74

2.14 Comparison of absolute difference in RMSE (\%) with an average density value (0.38) versus measured average individual tree density for live and dead (grey), live (white), and dead (black). Negative differences indicate improvement in RMSE. . . . . . . . . . . . . . . 76

2.15 Example of successful and unsuccessful cylinder fitting of two different tree point clouds. The successful scenario (left) was completed on a dead individual with low noise and branches are clearly accurately represented. The unsuccessful scenario (right) was completed on a living tree with dense foliage and high point cloud noise, resulting in unrealistic cylinder sizes withing the crown. . . . . . . .

2.16 Non-destructive estimates of biomass using a QSM approach on the (a) segmented trunk point cloud and (b) the entire unsegmented point cloud for living (black) and dead (open-circles) trees. . . . . .

3.1 Smithsonian Conservation Biology Institute SIGEO forest with stem map (green points) and terrestrial LiDAR plot locations (brown circles). TLS samples are color coded according to diameter and proportionally sized. Elevation is represented in the background (low: cool, high: warm). . . . . . . . . . . . . . . . 93

3.2 The top 10 dominant species contributing over $80 \%$ of biomass within the SIGEO forest as estimated using the Chojnacky et al. (2014) equations. [A] Absolute and [B] relative contributions to biomass. . . . . . . . . . . . . . . . . . 95

3.3 Models produced from the SimpleTree algorithm within Computree. Coregistered plot data [left] was used to produce plot-level models of tree structure [right]. 
3.4 Species specific allometry for $[\mathrm{A}]$ tree height predicted from diameter and biomass allometry derived from TLS models with [A] diameter, [B] untransformed diameter-height, and [C] log transformed diameter-height predictive models. Generalized allometry for each equation form is shown in dark grey. . . . . . . . . . . . . . . . . . 102

3.5 Height model validation with $[\mathrm{A}]$ general and $[\mathrm{B}]$ species specific equations. All trees used for validation were used to evaluate the generalized height allometry, while the species specific equation only compared species captured in the allometry. Species not represented in the general allometric model are black in color. Height estimates were made with a correction factor applied, as this equation had high sample size and high RMSE. . . . . . . . . . . . . . . . . . 104

3.6 Sensitivity of generalized height [top], diameter [middle], and diameterheight [bottom] models of biomass allometry. [left] $\beta_{0}$ and [middle] $\beta_{1}$ coefficients stabilize at approximately 180 samples for the diameter based model and 100 for the diameter and height model. The equation coefficients for the model excluding large trees (black) never reaches the benchmark coefficients (dashed line) of models including large trees. . . . . . . . . . . . . . . . . . . . . . 105

3.7 Comparison of percent error in biomass from the full allometric models using [A] height, [B] diameter, and height-diameter as predictors. Three scenarios were compared: 1) sample size-dependent error in the full model (blue), 2) sample size dependent error in the model excluding trees above $50 \mathrm{~cm}$ (red), and error of the model excluding trees above $50 \mathrm{~cm}$ from the full model (grey). The height equation excluding large trees deviated approximately 30\%, while diameter (10\%) and height-diameter (less than 1\%) were less affected by exclusion of large trees. The inclusion of large trees leads to less model error compared to increased sample size. . . . . . . . . 106

3.8 Comparison of [A] Chojnacky et al. 2014, [B] TLS height allometry [C] Jenkins et al. (2003), [D] TLS diameter allometry [E] corrected Jenkins et al. (2003), and [F] TLS diameter-height allometry biomass to TLS derived biomass for all tree models. . . . . . . . . . 107

3.9 Box-plot comparing range of observed RMSE (log units) for three TLS equation forms to Jenkins et al. 2003 RMSE. The Hard maple / oak / hickory / beech equation (blue) and Mixed Hardwood (red) equation RMSEs are indicated with the dotted horizontal lines. *Note: Chojnacky et al. 2014 equation RMSE was not reported so values could not be compared. . . . . . . . . . . . . . . 108 
4.1 Framework for reducing uncertainty with targeted TLS acquisition through modeling and improved allometry. At each step of biomass mapping TLS can potentially improve the current methodology. (A) At the stage of field sampling TLS is an efficient and relatively unbiased method of collecting standard forest structure information (e.g. DBH, height, basal area, vegetation area index). (B) Plot-level biomass estimation can be improved through direct stem modeling with TLS or non-destructive local allometry with TLS. (C) TLS can potentially improve sensor calibration and validation through full plot-level 3D reconstruction. Reduced uncertainty biomass allometry can be applied regionally, further improving sensor calibration. (D) Low uncertainty sensor calibration will provide the greatest potential for reducing uncertainty in biomass mapping. . . . . . . . . 126

4.2 Overview map of SIGEO forest at the Smithsonian Conservation Biology Institute in Front Royal,VA ( ). 14 randomly distributed $1 / 10$ th ha circular plots were located on the $20 \mathrm{~m}$ grid intersections and scanned with TLS (blue). 11 additional plots were included for LiDAR calibration (brown). An elevation model derived from the 2011 LiDAR acquisition shows the topography across the site. Note: TLS plots are not always centered over grid intersections as the TLS point cloud was georeferenced with the airborne LiDAR.

4.3 Comparison of LiDAR biomass calibration models (top row) and propagated uncertainty of biomass estimates (bottom row). Mean canopy height was used as a comparable canopy height metric collected using spaceborne LiDAR (GEDI or ICE-Sat II) (A \& D). LiDAR intensity was used as a comparable metric to radar backscatter, as collected using spaceborne PolSAR (B \& E). MCH and intensity were combined in the final model to emulate PolInSAR or TomoSAR (BIOMASS or NISAR) and potential cross-sensor fusion (C \& F). The TLS models (black) provided the lowest uncertainty estimates, followed by TLS allometry (grey), and Jenkins et al. 2003 allometry (red). TLS reduced the total propagated uncertainty of LiDAR calibration by over $10 \%$ (over $30 \%$ relative), with improvements in both plot-level and LiDAR calibration RMSE. Percentages within stacked bars indicate the relative contribution of uncertainty from allometry (blue) versus the LiDAR-derived empirical model (grey) . . . . . . . . . . . . . . . . . 136

5.1 Area-wide LiDAR-derived biomass estimates, based on mean forest canopy height and TLS calibration. The processed area (red) is part of a larger acquisition in Virginia (blue). . . . . . . . . . . . . 153 
5.2 Potential TLS sensor fusion approach, allowing temporal and spatial monitoring of ecosystem status over large area acquisitions. . . . . . 158

A.1 TLS scan of a lodgepole pine tree from Colorado State Forest selected for destructive sampling in Chapter 2. . . . . . . . . . . 165

A.2 Leaf-off upward view of plot 0628 taken March $25^{\text {th }}, 2015$. . . . . . 166

A.3 Leaf-on upward view of full color TLS scan in plot 0628 corresponding to previous hemispherical photograph. . . . . . . . . . . 167

A.4 1 ha South-facing transect at SCBI Large Forest Dynamics Plot. Image colored by intensity. . . . . . . . . . . . . . . . . . . . 168

A.5 LiDAR acquisition overlapping SCBI and used in TLS calibration.

Colored by elevation. . . . . . . . . . . . . . . . . . . 169 


\section{List of Tables}

2.1 Summary statistics for 21 destructively-sampled lodgepole pine ( $P i$ nus contorta) trees. Trunk biomass was measured for 22 trees. . . . 44

2.2 Summary statistics for non-destructive terrestrial LiDAR-derived parameters from 21 lodgepole pine (Pinus contorta) trees. Trunk biomass was estimated for 22 trees. RMSE (\%) is equivalent to CV(RMSE). . . . . . . . . . . . . . . . . . . 61

2.3 Comparison of the RMSE (\%) of the Outer Hull Model algorithm to other algorithms validated with destructive samples. Only trunk biomass RMSE \% was compared to minimize bias from higher uncertainty foliage estimates. . . . . . . . . . . . . . 65

2.4 Regression parameter values (standard error) for estimating foliage (Equation 1; $\mathrm{n}=90$ ) and wood (Equation 2; $\mathrm{n}=89$ ) wet mass for branches. . . . . . . . . . . . . . . . . 69

2.5 Calibration parameter values (standard error) for the OHM algo$\operatorname{rithm}\left(O H M_{\mathrm{P}}\right) \ldots \ldots \ldots \ldots \ldots \ldots \ldots \ldots \ldots$

2.6 Comparison of absolute difference in RMSE (\%) with an average density value (0.38) versus measured average individual tree density. Negative differences indicate improvement in RMSE. . . . . . . . 75

3.1 Summary statistics for all TLS modeled trees and predicted biomass. 100

3.2 General biomass allometry for 271 modeled trees. . . . . . . . . . . 101

3.3 Species-Specific allometry for the 10 most dominant species at the study site using height and diameter as predictors. Blank spaces indicate the same coefficient (top row in each section) was used across all species. . . . . . . . . . . . . . . . . . . 103

3.4 Species-Specific height allometry for the 10 most dominant species at the study site using diameter as the predictor. Blank spaces indicate the same coefficient was used across all species. . . . . . . 115 
3.5 Species-Specific volume allometry for the 10 most dominant species at the study site using diameter and height as predictors. Blank spaces indicate the same coefficient was used across all species. Note: the $\beta_{1}$ coefficient is identical to the biomass allometry coefficient.

4.1 Summary statistics for all trees modeled for TLS allometry. $\rho$ is based on the average wood density and not independently derived. . 134

4.2 General allometry including the 10 species modeled with TLS. Equations were created for Liriodendron tulipifera (Lt), Quercus (Q), and Carya $(\mathbf{C})$. The mixed hardwood equation (MH) includes Fraxinus americana, Liriodendron tulipifera, and Nyssa sylvatica. The Hard maple/Oak/Hickory/Beech equation (MO) includes Quercus, Carya, and Fagus grandifolia. RMSE is in log units and based on 5 -fold cross validation.

4.3 Uncertainty of nine empirical biomass models based on calibration data from TLS models, TLS allometry, and Jenkins et al. 2003 equations. Three models are compared using mean canopy height $(\mathrm{MCH})$ and LiDAR intensity, as well as a multiple regression using both metrics as predictors. Uncertainty from model residuals $\left(\sigma_{\epsilon, \text { plot }}\right)$ and propagated uncertainty $\left(\sigma_{\epsilon, \text { pred }}\right)$ are reported in $\mathrm{Mg} \mathrm{ha}^{-1}$ and as a percentage of the mean (equivalent to relative RMSE). . . 137 
For Adrienne, Moses, and Asa 


\section{Chapter 1}

Forest Structure and Understanding Global Carbon

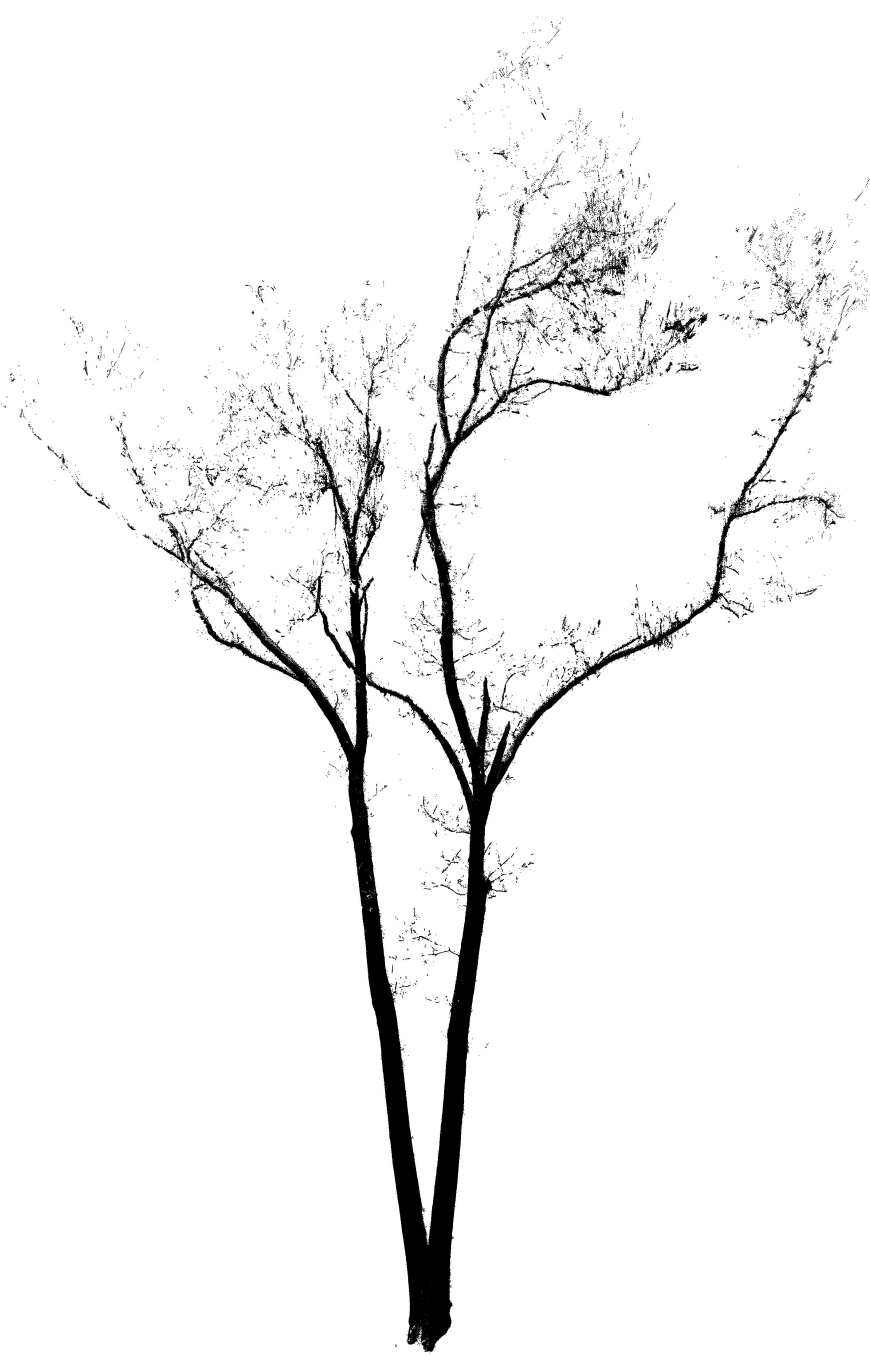




\subsection{The Role of Uncertainty in Understanding Forest Structure at Multiple Spatial Scales}

Uncertainty in forest biomass estimation is entirely dependent on plot-level estimates that rely on quality representative allometric equations. Developing local allometry is costly due to the laborious process of destructive sampling, increasing uncertainty when quality relationships are non-existent or more suited to broadscale applications for biomass mapping. Non-destructive estimates of biomass with terrestrial LiDAR can potentially improve plot-level estimates of forest biomass with the creation of local allometry, requiring a fraction of the time and cost of analogous traditional approaches. While several methods using terrestrial LiDAR have been proposed as suitable for biomass estimation, few use destructive samples for validation. Little is known of how these estimates will affect landscape level estimates of biomass using airborne LiDAR and other remote sensors. These considerations lead to a central question: Can terrestrial LiDAR reduce uncertainty in biomass mapping?

Trees are structurally complex organisms that provide a snapshot in time of the individuals life history. Long term changes in climate, disturbance events, and competitive effects are represented in the form of trees, but are difficult to quantify from a single observation. Structure is modulated according to deeper genetic effects that are the product of centuries of adaptation to a region. The limits on tree size are directly driven by all of these factors. In drylands, trees are shorter in stature (Nogueira et al., 2008), while moist climates are home to the tallest trees on Earth (Koch et al., 2004). Interactions between all of these factors are apparent when generalizing tree form and biomass with scaling relationships; what results is a high degree of uncertainty in tree structure that is exacerbated with large trees. A non-destructive method of measuring the structural complexity and biomass of trees is needed in order to improve existing allometry.

When trees are considered across the landscape as a forest those driving characteristics change. Important factors to the individual tree are not of great consequence to the entire forest. Competitive effects transition to gradients in moisture and light. Landscape-scale variations in structure are best investigated with re- 
mote sensing. Two- and three-dimensional measurements from aircraft and space provide the means to ask unprecedented questions about forest structure. Global distributions of biomass have been created, but uncertainty pervades and disagreement in maps is common in high biomass locations, due to local variations in tree structure. As these large-scale mapping efforts continue, uncertainty reduction must be focused on the application of locally derived allometry. A nondestructive method of biomass retrieval will provide the means to reduce uncertainty in biomass distributions across the landscape and the globe, providing a greater understanding of the large-scale factors that influence structure.

The scale at which we observe a forest clearly impacts the way it is understood. A single tree interacts with direct competitors, altering the structural allocation of carbon. Though competition impacts all trees, the forest stand is driven more greatly by environmental gradients. Using traditional methods, these variations have been documented, but, with the advent of terrestrial LiDAR as a means to non-destructively measure forest structure, these variations can be quantified with immense detail in three dimensions. This presents an opportunity to determine how plot-level applications of local allometry alter landscape level maps of forest structure. For global biomass uncertainty reduction this offers a framework for the creation of local allometric equations that will improve estimates in areas where values are commonly divergent.

\subsection{Forests in the Global Context}

Rising atmospheric $\mathrm{CO}_{2}$ levels due to anthropogenic fossil fuel combustion are expected to produce significant warming over the next century (IPCC, 2006). Concerns about the consequences of human interactions with the carbon cycle have uncovered the need to understand carbon storage and dynamics with greater detail (Schimel, 1995). Terrestrial carbon is primarily located belowground in organic soil and aboveground in the biomass of the worlds forests (Schlesinger \& Bernhardt, 2013b). Photosynthesis in forests, as well as other vegetated ecosystems, drives the interannual carbon dynamics and the removal of $\mathrm{CO}_{2}$ from the atmosphere - as made clear by the striking latitudinal differences in magnitude (Conway et al., 1988) produced by the $2 / 3$ rds of terrestrial vegetation in which growth 
is seasonal (Box, 1988). As carbon dioxide is removed from the atmosphere by physical or biological means, these sinks reduce the climate forcing caused by the radiatively-active gas, effectively cooling the atmosphere, on average (Schlesinger $\&$ Bernhardt, 2013a). It follows that in order to confidently predict the effects of rising $\mathrm{CO}_{2}$ concentrations on the planet, understanding the fluxes of the carbon cycle is imperative.

Several of the fluxes in the carbon cycle are known with a high level of certainty because of the physical nature of these processes. Driven by diffusion, oceans act as a sink for a portion of atmospheric $\mathrm{CO}_{2}$ as the gas is converted to carbonic acid and calcium carbonate (Schlesinger \& Bernhardt, 2013b). Of these components, only a small fraction are permanently buried and removed from the carbon cycle. Atmospheric concentration and dynamics can be measured reliably in the free-atmosphere, as shown in the well-known Mona Loa observations (Keeling CD et al., 1995), remaining relatively stable due to the long residence time of $\mathrm{CO}_{2}$, with variations primarily caused by glacial and interglacial periods from Milankovitch Cycles. However, since the Industrial Revolution, carbon dioxide concentrations have increased sharply (Barnola et al., 1995). The atmospheric trend from these observations has made clear the strong positive relationship between fossil fuel emissions and atmospheric $\mathrm{CO}_{2}$ concentration - as combustion has increased, so have carbon dioxide concentrations, and thus the climate forcing (Mann et al., 1998). As confirmation, the proportion of fossil fuel derived $\mathrm{CO}_{2}$ has been determined using isotopic analysis of atmospheric dilution from carbon depleted of C14 (Keeling, 1979). Though not a natural physical process, fossil fuel emissions are regularly estimated with reasonable certainty.

Unlike the physical processes in the carbon cycle, biophysical processes and anthropogenic influences drive the remaining fluxes of the carbon cycle: land use change and the terrestrial sink estimated to be $2.2 \pm 0.8$ and $-2.9 \pm 1.1 \mathrm{PgC} \mathrm{yr}^{-1}$ respectively (Houghton, 2003). Land use change depends on human interaction and disturbance processes, both of which are highly variable on an annual basis. The primary issue for quantifying land use change is monitoring, a problem that can likely be solved with remote sensing (Asner et al., 2014). The terrestrial sink has been estimated using a subtractive mass balance approach, but this method is not directly derived and results in extreme variability as it is driven by all other 
estimated processes (Canadell et al., 2007). Other independent estimates indicate the northern hemisphere is the primary location of the terrestrial carbon sink (Fan et al., 1998) and the productivity of forests in this part of the globe fuel the sink strength (Pan et al., 2011). Yet, uncertainty is still pervasive in these estimates differing methods of estimating the magnitude of the terrestrial flux may not even agree if the flux is positive or negative (Houghton, 2005).

While these trends in carbon dynamics are useful for determining the potential of forests to remove carbon from the atmosphere, they reveal little about the storage and distribution of carbon across the land surface. The United Nations Framework Convention on Climate Change (UNFCCC) has pushed to improve monitoring of forest cover for Reducing Emissions from Deforestation and Forest Degradation (REDD+). The three tier system implemented in these assessments of $\mathrm{C}$ storage are categorized specifically according to the level of uncertainty and level of detail within the estimates (IPCC, 2006). Many of the initial goals of REDD+ could be addressed and much of the uncertainty surrounding the terrestrial flux could be reduced with a quality baseline carbon map (Houghton et al., 2009). Global biomass carbon density distributions have since been created with ICESat GLAS LiDAR data (Saatchi et al., 2011; Baccini et al., 2012). However, these maps have poor agreement and uncertainty estimates are not as low as originally estimated - a finding thought to be attributed to variations in wood density and allometric relationships (Mitchard et al., 2014).

In order to reduce the uncertainty of global forest biomass it follows that a combination of improvements will be needed at every scale of measurement from the individual tree to the continental scale. Quality plot level measurements are essential for baseline biomass maps, as they represent the only true values that will validate and drive all other inferred values. Of these plot level measurements, improvements in allometric biomass estimation and wood density will provide the greatest boost in confidence at all scales (Mitchard et al., 2014). Plot level estimates can successfully be scaled to the landscape using LiDAR in combination with other environmental variables (Asner et al., 2012), but success depends on the incorporation of the variability of wood density and allometry. Global-scale estimates of biomass have the potential to be greatly improved with data fusion approaches (Mitchard et al., 2012), especially considering the most recently planned satellite 
launches that will use both radar (Le Toan et al., 2011) and LiDAR (GEDI and ICESat-2). However, as with detailed mapping across smaller areas, both types of satellite remote sensing platforms will require high confidence ground-truthing locations for calibration (Hall et al., 2011). It is clear that improvements in global biomass carbon estimation will only come from improvements in the methodology and technology used at each scale of measurement, so the current range of successful methods of biomass mapping must be evaluated.

\subsection{Traditional Methods of Biomass Estimation and Limitations}

Traditional techniques of determining the total quantity and distribution of forest biomass carbon rely on extensive networks of field-based measurements (e.g. the Forest Inventory and Analysis (FIA) plots that exist in the United States). At each plot, information on tree diameter at breast height (DBH) and height are measured. Species-specific or plant functional type-specific relationships between diameter, height, and biomass are determined with a small number of destructive samples and applied to the field measurements (TerMikaelian \& Korzukhin, 1997). Plot-level data can be aggregated to create maps of the spatial distribution of biomass over large areas (Brown et al., 1999; Jenkins et al., 2001).

Biomass mapping hinges greatly on a complex destructive sampling process that is fraught with difficulties - invariably leading to measurement errors and increased uncertainty. In the simplest form, destructive sampling requires the weight of the entire tree and a number of subsamples of the main components of the tree that can be dried (Brown, 1997). Tree components are dried in order to determine the dry biomass, reducing error due to variation in water content in the wood. The entire process can take a number of weeks or months to complete, depending on the wood characteristics. At each step there is a potential for measurement error, from the time the tree is felled to the drying of subsamples. Field equipment may be in poor condition or weighing may not be completed correctly. In some instances, tree components may not be measured at all. At the drying stage, residual water content from insufficient drying may bias measurements. 
Destructive sampling is made more difficult in areas of extreme topography and climate, where the work becomes dangerous, vulnerable to measurement error, or impossible to complete. In many countries, political or social factors play a major role in when and where harvesting can take place. For example: sampling in protected areas is often strictly prohibited due to cultural significance. All of these factors contribute to uncertainty from reduced sample sizes and bias from an uneven spatial distribution of destructive sampling locations.

Measurement error is not always a major component of the total uncertainty of allometric relationships, but spatial bias is. Often these relationships are created on the national-scale in order to allow for a single or limited number of equations that are applicable to the largest area possible (Chave et al., 2005). Tree growth form varies greatly due to the unique life history of each individual that has been exposed to a combination of environmental factors: climatic, topographic, intraand interspecific competition, and unexplainable variation. A trees capacity to change growth characteristics is essential for maximizing success under situations that are not optimal - the end result being a degree of individuality that makes generalization of allometry difficult. For this reason, the aforementioned allometric relationships are often not applicable or not advisable to be used at local scales (Jenkins et al., 2003) and, in most instances, using a locally- or regionally derived equivalent is preferable for reducing spatial bias (TerMikaelian \& Korzukhin, 1997).

Under ideal conditions of low measurement error and the availability of a locally derived allometric relationship, applications beyond the measured diameter or height range will have high uncertainty. Since the limitations of destructive sampling are so great, the largest trees in forests often go unmeasured, resulting in a limit to the applicability of these relationships (Brown, 2002). The vast majority of allometric equations for hardwood species have a maximum of around 70-90 cm (Chojnacky et al., 2014), but greater diameter trees are frequently observed in plot measurements and allometric equations are still applied. This poses a problem for plot-level estimates of biomass where large trees drive the differences in biomass. On the stand-scale, trees greater than $70 \mathrm{~cm}$ DBH account for between 30-40\% of total aboveground biomass (Brown, 1997). If the uncertainty of these large trees is not reduced, estimates of biomass across the landscape have the potential to be drastically over- or underestimated. 


\subsection{Remote Sensing for Biomass Estimation}

Remote sensing has become the most ideal method of extrapolating plot-level biomass estimates to the landscape. The very basis of remote sensing relies on the remote observation of the emission or reflectance of the area of interest. Sensors may be passive relying on the illumination of the surface by solar radiation and sensing this reflectance or they may be active illuminated by a source of energy located near the sensor. The reflectance, under both sensor types, depends on the spectral reflectance of the vegetation and the atmosphere, since the measurement includes both components. A two-dimensional grid of reflectance characteristics is produced, essentially equivalent to a digital photograph, with each pixel representing an intensity of reflectance over a known geolocated area. Active sensors can add an extra dimension of vertical structural measurement that helps improve biomass estimates (Shugart et al., 2010). Each platform type may be preferable under specific requirements and limitations surrounding biomass mapping, depending highly on the level of detail required, the standing biomass density, the climate of the study area, as well as a whole host of other considerations. Reflectance and measures of forest structure are used along with the plot-level field measurements to model their relationship for biomass estimation across the landscape.

\subsubsection{Passive Optical Remote Sensing}

The variation in horizontal forest structure across the landscape is clear from any view above the forest canopy. This structural heterogeneity is closely linked to the biomass of a forest stand and is the primary determining factor of estimating biomass using passive optical remote sensors. These sensors vary widely in resolution, from sub meter (Quickbird) to $30 \mathrm{~m}$ (Landsat) to $250 \mathrm{~m}$ (MODIS) to $1 \mathrm{~km}$ (AVHRR). The scale of study and phenomenon of interest will determine which resolution is ideal in any biogeographical study (Delcourt et al., 1982), but availability of specific bands and measurement frequency should also be considered. Global and long-term biomass dynamics have been estimated with the lower resolutions of AVHRR and MODIS, while the higher resolution options are used for detailed regional or local scale applications. 
Biomass has been estimated using empirical regression (Hall et al., 2006), nonlinear parametric analysis (Baccini et al., 2004), and crown allometry (Soenen et al., 2010) using models of simple reflectance or measurements of canopy attributes. Reflectance is frequently converted to Normalized Difference Vegetation Index (NDVI) and can be used to estimate biomass effectively, but will saturate at high values and under dense vegetation. LAI is strongly correlated to biomass and can be estimated with optical sensors (Myneni et al., 2002). By modeling the reflectance of a forest canopy in three dimensions, estimates of crown size and density can be made - both of which allow for biomass assessment (Li \& Strahler, 1985). Shadow fraction, representing a combination of shaded crown and ground, has also been found to be proportional to biomass (Greenberg et al., 2005). Incorporating environmental gradients such as elevation can further improve all of these methods. Crown allometry methods apply plot-scale measurements to remote sensing data by approximating canopy geometry - shadow fraction (Soenen et al., 2010), crown diameter (Palace et al., 2008), or foliage biomass (Zhang \& Kondragunta, 2006) - and linking this information to existing allometric relationships. Finally, species identification with hyperspectral sensors has the potential to improve existing optical biomass mapping methods.

\subsubsection{Radar Remote Sensing}

Synthetic Aperture Radar (SAR) is an active sensor type that allows for a more complex view of forest structure than passive optical sensors. Radar emits a pulse in the microwave spectrum that scatters off vegetation and returns as a characteristic representation of the area of interest. Radar measurements must be made off-nadir, so images will always contain some degree of shadowing due to the slanted view angle. Radar is of particular interest in tropical, or other areas with frequent cloud cover, because of the relative insensitivity to atmospheric moisture. While radar is not a new technology the applications in forestry are only beginning to be explored.

Biomass estimation using SAR has been approached by using information on the backscatter, coherence, and phase of the returning pulse (Koch, 2010). Methods using backscatter - essentially providing a two-dimensional map of the inten- 
sity of the backscatter - can effectively characterize areas of lower forest biomass, but quickly saturate in the presence of high biomass (Rauste, 2005). Coherence methods rely on SAR interferometry - multi temporal radar data at differing view angles - to detect temporal change and the height dimension (Wegmuller \& Werner, 1995; Bamler \& Hartl, 1998). Satellites like the scheduled BIOMASS mission (Le Toan et al., 2011) rely on the wavelength of the emission pulse and polarization of scattering in order to determine biomass (Le Toan et al., 1992). Longer wavelengths (L- and P-bands) are capable of capturing larger forest attributes such as trunks or large branches and have been found to be strongly linearly correlated to forest biomass (Saatchi et al., 2007), but saturate at high levels (greater than $200 \mathrm{Mg} /$ ha with P-band). Issues with saturation can be addressed with Polarimetric SAR Interferometry (Pol-InSAR) by estimating forest height in order to extend the usable biomass estimation range.

\subsubsection{LiDAR Remote Sensing: Technological Overview and Biomass Mapping}

Light Detection and Ranging (LiDAR) provides extremely high-resolution data that is useful for quantifying biomass and forest structure over large areas (Lefsky et al., 1999). LiDAR technology functions by measuring the distance between the sensor where a laser pulse is emitted and target of interest, by precisely measuring the time between the emission and detection of the reflected return pulse (M. A. Lefsky et al. 2002). Distance between the sensor and target is determined by:

$$
d=(t c) / 2
$$

Where, $\mathrm{t}$ is time elapsed and $\mathrm{c}$ is the speed of light $\left(299,792,458 \mathrm{~m} \mathrm{~s}^{-1}\right)$. This essentially uses the constant speed of light to determine a distance with nanosecond time differences. LiDAR instruments are often flown in aircraft in order to measure topographic features over a wide area. In forested environments the laser intercepts the canopy proportionally to the density of vegetative cover, resulting in a mixture of canopy and ground returns. The measured difference between these two classified returns is useful in determining average canopy height of a forest 
with a resolution equivalent to the spacing of returns in the data.

The two main categories of LiDAR sensors are discrete-return and waveformrecording. Discrete-return sensors may measure only one return or multiple returns by using waveform analysis to determine interception with a solid object. Information such as the leading edge of pulse peaks, peaks, and, often, the peak intensity is recorded a product of the reflectance of the object at the laser's wavelength, the portion of the beam intercepted, the incidence angle of the object, and the power of the emitted laser pulse. Waveform-recording sensors record the intensity of the waveform as it varies throughout the acquisition time over the measurement area. Both categories of sensors record essentially the same information, however, waveform-recording provides the raw uninterpolated data as the end product. When this data is combined with Global Positioning System (GPS) data that measures position in space and Inertial Navigation Systems (INS) that measure the attitude of the laser, the exact location of each return can be determined. Discrete-return LiDAR is ideal for detailed mapping of topographic features due to the high pulse rate and small diameter of the laser beam. This advantage over waveform sensors makes it ideal for imaging of complex forest canopies and delineation of individual tree crowns. Since waveform-recording systems display a continuous stream of data from a constant beam source and the beam diameter is generally much larger, they are ideal for measuring the structural characteristics of forest canopies over a large area.

LiDAR has become the standard for accurate large-scale mapping efforts in forestry (Lefsky et al., 2002). The most common approach is a two-stage procedure (Naesset, 2002) that relies on many field inventory plots that are located within the range of the LiDAR data. Structural measures such as basal area or biomass are summed over the plots and related to the structure of the LiDAR point cloud. LiDAR returns are statistically analyzed and decomposed into height percentiles, cover estimates, as well as canopy height models. In tropical systems, where detailed plot measurements are sparse, generalized allometric equations for biomass density have been related to a mean canopy height (Asner, 2009). Similar methods have been used in conjunction with spaceborne LiDAR to estimate global canopy height (Simard et al., 2011) and biomass (Saatchi et al., 2011). Management applications are also common ranging from fire fuel estimation (Andersen 
et al., 2005) to habitat characterization (Nelson et al., 2005). High density small footprint LiDAR can used measurements of crown diameter to allometrically estimate biomass of whole forest stands (Popescu et al., 2004). Alternative functional models of the canopy from high density LiDAR are useful for low RMSE biomass estimates (Zhao et al., 2009). Return intensity can be useful in differentiating between forest types (Garcia et al., 2010), but fusion of hyperspectral data to LiDAR allows for further improved biomass estimation by approximating species assemblages in broadleaf and coniferous forests (Popescu et al., 2004). Many of these methods of biomass mapping provide detailed structural information that will be used to verify and validate models of global forest dynamics (Shugart et al., 2015).

\subsection{Terrestrial LiDAR in Forestry}

Terrestrial LiDAR or Terrestrial Laser Scanners (TLS) function by the same basic concept surrounding airborne LiDAR or airborne laser scanning (ALS), relying on the minute differences in laser pulse return timing or shift in phase between a continuously emitted and received laser beam. However, instead of moving over the forest landscape, these scanners are stationary, requiring a different analysis approach. The range specifications for most TLS units is on the order of 100-500 $\mathrm{m}$ and, since the objects within the scan area are much closer to the beam source, density of the resulting point cloud is orders of magnitude higher than ALS over a smaller area of interest. For instance, a typical ALS point cloud may have an average horizontal point spacing of $1 \mathrm{~m}$, while the point spacing of a TLS point cloud may be close to $1 \mathrm{~cm}$. This significant increase in point cloud density results in the resolution of fine scale structural information, not only on the plot scale, but also on the individual tree scale. Similarly, accuracy of TLS is on the order of several millimeters, while ALS data ranges from a few centimeters in the vertical to a meter in the horizontal. A plethora of approaches have been used to interpret the immense quantity of data from this novel technology for forestry mensuration purposes. 


\subsubsection{Traditional Forestry Measurements with TLS}

Tree diameter at breast height $(\mathrm{DBH})$, height, and location are the basic fundamental measurements of a forest. Terrestrial LiDAR potentially presents the opportunity of accelerating the speed and quality of these measurements. Automatic diameter measurement, tree separation, and stem mapping are common applications of TLS (Tansey et al., 2009). Early studies using ground-based LiDAR focused primarily on extracting these measurements (Hopkinson et al., 2004). TLS captured over $95 \%$ of the stems that were manually measured, indicating this technology could be a suitable and less biased method of creating stem maps. Stem maps of over 200 trees were created with similar algorithms on a leaf-off plot (Brolly et al., 2009). They found a 2D multiple height circle-fitting algorithm outperformed the cylinder-fitting algorithm, having a lower RMSE, due to the independence of the circular shapes, however the cylinder method was able to capture non-vertical stems, which may be of use when modeling crown branch structure (See Section 1.4.3). Improvements can be made leading to automation by reducing the effects of topography or noise by creating terrain and canopy models. Similar to airborne LiDAR, TLS data can be normalized by topographic differences by creating a Digital Terrain Model (DTM) from the point cloud data (McDaniel et al., 2012) converting the z-coordinates to height above ground. This method has simplified the process of finding the appropriate level to measure DBH and has lead towards automation. The addition of a Canopy Height Model (CHM) is useful for reducing the presence of points not associated with solid objects (e.g. noise from low intensity returns) (Henning \& Radtke, 2006b). Moreover, a topographic correction is necessary when analyzing the vertical profile of vegetation or this distribution will not be representative of the true structure of the forest (Calders et al., 2014) (see Section 1.4.2).

Separation and detection of individual trees from surrounding objects can be accomplished with a Hough-transformation and stem diameter can be measured with either a circle- or ellipse-fitting algorithm (Aschoff \& Spiecker, 2004; Tansey et al., 2009; Chmielewski et al., 2010). Similar approaches reported RMSE on the order of $1.5 \mathrm{~cm}$ and $80 \mathrm{~cm}$ for DBH and height respectively (Bienert et al., 2006a). These algorithms were used to compare the advantages and disadvantages of using 
multiple scanning locations in order to reduce occlusion effects finding the major tradeoff being processing time or measurement accuracy (Bienert et al., 2006b). In a collaboration with the company TreeMetrics, these developments were compiled into a program, AutoStem ${ }^{\mathrm{TM}}$, which automatically computed inventory parameters within managed forests (Bienert et al., 2007). All of these algorithms were validated against field measurements in a pilot study that showed promise for the technology, but indicated the need for further developments before being operational (Maas et al., 2008). Others have attempted to develop the technology and apply it to mobile mapping with UAVs with relatively successful results under extremely controlled conditions, further reducing the effort required to acquire these forestry measurements (Jaakkola et al., 2010; Rutzinger et al., 2010). Recently, stem mapping with TLS was accomplished with greater than $80 \%$ detection rate on a plot of bamboo with a stem density of over 7,500 stems/ha (Xia et al., 2015). As algorithms have improved, automation has become the main focus, making terrestrial LiDAR a reliable alternative to traditional forestry methods (Liang \& Hyyppa, 2013; Yang et al., 2016). All of these studies are representative of many of the other approaches for measuring these particular tree-level attributes (Hosoi et al., 2005; Liang et al., 2008).

Outside of cylinder or circle-fitting, intensity of the return laser pulse can be used as a reliable variable to estimate DBH within the three-dimensional point cloud (Lovell et al., 2011). The intensity of the reflected laser pulse from TLS across a forest transect can fit a simple model based on the range and diameter of the tree trunk. The basic concept relies on the curvature of the tree trunk - the intensity of the returns vary proportionally to the trunk of the tree, decreasing as it curves less and less perpendicular to the laser source. This suggests that adding intensity as a validation metric in DBH measurement could significantly improve the usefulness of TLS data. A similar approach using range imagery analysis can be used to provide automatic TLS point cloud registration of multiple scans by using the approximated central trunk location along the $\mathrm{x}-\mathrm{y}$ axis for each tree of interest, rather than relying on artificial registration targets (Henning \& Radtke, 2006a). Once registered, diameter can be measured and compared to manual measurements of the tree trunk. Error increases with height above ground, due to the higher upper canopy density and occlusion effects. Registration of multiple 
scans allows for a more detailed examination of the structure of the forest in the three-dimensional data (Henning \& Radtke, 2006b).

\subsubsection{Vegetation Structure: Gap Fraction and Voxel Ap- proaches}

Vegetation structure measurements (e.g. foliage profiles) are essential for understanding many aspects of a forest, but traditional methods are extremely labor intensive, requiring elaborate field setups (MacArthur \& Horn, 1969). The technique essentially involves many strings extending vertically throughout the plot. If a leaf is intersected the height aboveground is noted and the process is repeated until the plot area is measured. The acquired measurements can be used to approximate the distribution of foliage throughout the plot. If one can imagine laser beams that measure distance replacing the elaborate number of strings, the transition to a more modern approach is clear. Terrestrial LiDAR units emit millions of these beams that each measure the distance of any object they intersect - vastly improving the number and quality of these measurements.

Estimation of vegetation profiles in the form of plant area index (PAI) or leaf area density (LAD) is commonly accomplished with the use of a gap probability and gap fraction. This method was originally used in airborne LiDAR studies with great success and then adapted to terrestrial LiDAR in order to account for the range of inclination angles present (Lovell et al. 2003). The basic premise draws from MacArthur and Horn in the types of measurements, but the conversion must be made between the measured ranges from the scanner to heights above ground. For terrestrial LiDAR, the gap probability can be defined by 1 minus the ratio of the number of laser interceptions at a particular height above ground to the total number of emitted pulses. The fraction at any particular point in the canopy is equal to the gap probability. It follows that, as height aboveground increases the overlying vegetation will decrease. Thus, a gap probability function is often highest at the top of the canopy (i.e no vegetation) and lowest at ground level. The gap probability at the ground level will be proportional to the total vegetation cover above that point, often expressed as leaf area index (LAI). The conversion between gap probability to LAD or PAI is made by approximating the first derivative of 
this curve (Jupp et al., 2008). The first derivative represents the shape of the vegetation profile because the rate of change in gap probability is proportional to the presence of vegetation in the path of the emitted laser pulse. This method has be used in many studies analyzing forest structure (Danson et al., 2007; Jupp et al., 2007), seasonal changes (Hosoi \& Omasa, 2009a; Calders et al., 2015a) and leaf bulk density (Pimont et al., 2015). In comparison with traditional hemispherical photography (Chen et al., 1991), the gap probability method in conjunction with TLS was found to be as effective at estimating LAI, while simultaneously providing three-dimensional structural information (Seidel et al., 2012).

Voxelization is useful for reducing the variable point density effects of TLS and has been applied in many investigations of forest structure. This process analyzes adjacent points in space, orders via a grid structure, and produces a set of voxels - evenly-spaced points in space. Voxels can be used to examine the frequency distribution with respect to height of TLS and ALS returns in order to infer LAD from the point cloud (Chasmer et al., 2004). Using a scanner with a narrow field of view placed in symmetrical locations around two different trees, Hosoi and Omasa (Hosoi \& Omasa, 2006) were able to accurately model LAD and LAI profiles using voxels. Leaf-on and leaf-off tree voxel models were created for both species and used as a means to separate photosynthetic tissue from non-photosynthetic tissue in the TLS point cloud. This work notes the importance of a priori knowledge of leaf inclination distribution of the trees being measured when determining LAI, however this measure is extremely time consuming to complete across the entirety of the tree. When the laser zenith angle, $\theta$, is $57.5^{\circ}$ the correction for leaf inclination is almost independent of leaf inclination: $\alpha(\theta) \approx 1.1$. Thus, all returns at this this so-called hinge angle can be used to determine cumulative LAI of the tree. The primary source of error from using the aforementioned methods is the presence of non-photosynthetic tissue in the final leaf-only point cloud. The error associated with TLS-derived LAD measurements was reported to range from $9.5 \%$ - 37\%, depending primarily on the measurement method and location (Hosoi \& Omasa, 2007). Omasa et al. (2006) integrated this type of processing into existing methods of ecological remote sensing to show a range of potential applications of the TLS data such as determining canopy height, canopy structure, and carbon stocks. They then combined the $3 \mathrm{D}$ data with high resolution imaging techniques 
to determine leaf-level information including chlorophyll florescence, photochemical reflectance index, and leaf temperature. This type of data fusion was also used to combine airborne and terrestrial LiDAR in order to model species of coniferous trees (Omasa et al., 2008) and broadleaved species (Hosoi et al., 2010). Alternatively, similar analysis steps prove to be equally effective in estimation of plant characteristics when applied to other plant types, such as grasses (Hosoi \& Omasa, 2009b). Determination of plant area index (PAI) was completed in a similar fashion to Chasmer et al. ( 2004) using a voxel-based ray tracing method that summarizes the total volume of aboveground material that occupies the point cloud relative to the total scan area. Leaf area index (LAI) was determined by subtracting the leaf-off voxels from the leaf-on voxels and summarizing the horizontal voxel density over the plot area.

\subsubsection{Biomass and Volume Retrieval: Voxel Approaches}

Voxelization is not only useful for vegetation profiling, but can also be used to estimate volume and biomass of the woody portion of the tree (Hosoi \& Omasa, 2006). By ordering the points in a 3D grid structure the produced set of voxels represent defined volumes of space within the point cloud (Meagher, 1980; Hinks et al., 2012). Each voxel cell may be given a quantifiable volume, depending on the presence or absence of points within the point cloud (Moskal et al., 2009). Voxelizing also retains point density information from the original point cloud and can be useful for distinguishing between different structures in the cloud. This very simple process allows for a relatively quick and highly detailed estimation of volume in forested areas.

Creating detailed solid volumes from voxel based analysis is inherently difficult due to the lack of infilling within the woody portion of the trees being measured. Thus, all woody volume estimates derived from the voxel method will be underestimated with any trees that have trunks larger than the specified voxel size. One method of infilling the point cloud and creating solid volumes is to connect the outer voxels by way of Moores neighbor tracing algorithm (Ghuneim, 2009). This algorithm functions by utilizing the Moore neighborhood, or the set of 8 pixels that share a corner or edge with that pixel. In a clockwise direction, the algorithm 
encircles a cluster of pixels or, in 3 dimensions, voxels connecting the outer points until the origin of the algorithm is reached. This creates a totally enclosed contour of the voxels being analyzed. Any empty voxels within this contour can be filled and added to the final volume determination. This process is repeated at even intervals moving from the bottom to the top of the tree and the entirety of the tree is filled with voxels representing wood volume. In order for accurate volumetric estimates to be made on the smallest branches voxel size must be related to the laser beam diameter and average smallest branch size (Hosoi et al., 2013b). By summing the volume at each vertical section the distribution of biomass can be observed and compared directly to calculated LAD (Hosoi et al., 2013a).

A bounding box method has been shown to provide higher accuracy estimates of wood volume because there is little overestimation since the enclosed area is defined by the original TLS returns (Bienert et al., 2014). Infilling methods often rely on the interpolated exterior of the tree stem to act as an indicator for internal space, external space, and noisy points or branches that do not require infilling (Bienert et al., 2014; Hosoi et al., 2013a). The algorithms search each column of voxels along the $y$-direction for a filled voxel. Once found, the entire column is checked from Ymin $<\mathrm{Y}<\mathrm{Ymax}$ and once a filled voxel is encountered a counter is increased by 1 for that column. The process is repeated along the $\mathrm{x}$-direction, the result of which are labeled potential voxel infilling candidates for all voxels that have been marked twice or have a count of 2 . A second verification of these voxels is made by searching the voxels nearest neighborhood with a radius no larger than half of the largest stem diameter. Filled voxels or voxels with a count of 2 within the immediate neighborhood are considered as the interior.

\subsubsection{Biomass and Volume Retrieval: Primitive-fitting Ap- proaches}

Volume and biomass are two of the most complex measurements derived from noisy TLS point cloud data. Voxelization methods can lead to a host of problems if applied incorrectly and in order to perform as expected infilling must be a key component of any algorithm. Fitting of cylinders or other three-dimensional shapes (i.e. primitives) to the point cloud will circumvent many of the issues 
surrounding voxelization methods. Most primitive-fitting methods iteratively fit a shape to the points in the point cloud - in a least-squares sense - building from the bottom to the top of the tree. Small height intervals are often used to suppress unexpected fitting and overestimated cylinder size (Pfeifer et al., 2004). If the central trunk scan is of high quality and the trunk structure is not cylindrical, other non-cylindrical fitting methods may be used that are more representative (Pfeifer \& Winterhalder, 2004; Hildebrandt \& Iost, 2012; Feliciano et al., 2014; Olagoke et al., 2016). A hybrid cylinder-voxel method has also been used on a variety of complex tree structures when branches are too difficult to model directly (Lefsky \& McHale, 2008). Multi-scan plot design is common in modeling for biomass or stand volume, but single-scan methods can provide acceptable results if a correction factor is considered for occluded trees (Ducey \& Astrup, 2013; Astrup et al., 2014)

Cylinder fitting methods outweigh other methods greatly in their application prevalence due to the availability of cylinder fitting algorithms that exists in several software packages (Dassot et al., 2012), but semi-automated (Schnabel et al., 2007) and fully-automated algorithms (Raumonen et al., 2013) are becoming more common. Validation of cylinder fitting available in software packages with destructive samples suggests that these methods provide an acceptable non-destructive estimate of biomass, as long as scan resolution can accurately represent branches (Dassot et al., 2012). Automated methods like Quantitative Surface Models (QSMs) (Raumonen et al., 2013) have been applied to accurately estimate individual tree biomass (Calders et al., 2015b), but similar validation studies are not completed routinely. Validation using parameters that are more easily measurable in the field (e.g. stem diameter) are more often used due to reduced cost and labor expenditure (Hackenberg et al., 2014; Liang et al., 2014). Open source versions of automated QSMs are being made available and are in development currently (Hackenberg et al., 2015). These algorithms are being used to investigate large forest areas with a radiative transfer model by modeling every tree in both leaf-on and leaf-off conditions (Calders et al., 2016). This approach is even applicable for volume quantification of root structures (Smith et al., 2014).

The most visually complex versions of cylinder fitting approximate every structure of the modeled tree from trunk to foliage using computer vision techniques (Côté et al., 2011). Though this approach similarly relies on cylinder fitting meth- 
ods the primary function uses the point cloud as a guide for estimating and recreating tree structure based on generalized tree form, rather than recreating an entirely accurate model of the tree of interest (Côté et al., 2012). Several field measurements are required in order to parameterize the model, but this method reduces errors due to occlusion of trunk shapes and models foliage in great detail, which is otherwise not currently possible. This method has been proposed as ideal for complex investigations of three-dimensional radiative transfer of individual trees and forests (Côté et al., 2009).

\subsection{General Knowledge Gap and Main Questions}

Terrestrial LiDAR represents the smallest-scale and most detailed forestry measurement tool, but the potential for these measurements to be applied across the landscape is great. Although there has been an explosion of work aimed at addressing the potential of TLS for forestry measurements, the range of applications are still limited and many questions exist. Very few studies have validated TLS biomass estimates against destructively harvested data - an essential component of modeling studies that increases confidence. Exploration of the implications of applying alternative TLS allometry to plot or stand level forest biomass estimates are non-existent and essential information that will allow the technology to become operational. Moreover, the relationship between remote sensing estimates of biomass from radar or LiDAR and those estimates using terrestrial LiDAR are unknown. These several knowledge gaps form a scale-driven set of questions that will be addressed and investigated:

[1] Can terrestrial LiDAR be used to estimate biomass at the individual tree scale?

[2] How does locally derived terrestrial LiDAR allometry influence estimates of biomass compared to the more commonly used allometric relationships?

[3] Can terrestrial LiDAR directly reduce uncertainty in sensor calibration, validation, and biomass mapping through plot-level $3 D$ modeling and non-destructive allometric equations? 


\subsection{Dissertation Structure and Study Motivation}

The four stated questions will be addressed throughout this dissertation in the following chapters:

Chapter 1: Forest Structure and Understanding Global Carbon

Chapter 2: Non-Destructive Biomass Estimation from Terrestrial LiDAR

Chapter 3: Implications of Applying Non-Destructive Local Allometry

Chapter 4: Improved Biomass Calibration with Terrestrial LiDAR

Chapter 5: Moving Forward with Terrestrial LiDAR

The introductory chapter provides the foundational knowledge for the subsequent investigations. Chapters 2-4 provide a scale-driven analysis - providing insight into the potential of targeted application of terrestrial LiDAR to improve landscapescale carbon estimates. The final chapter is a reflection on the findings of this dissertation and the next steps for terrestrial laser scanning in the context of forestry.

\section{Chapter 2: Non-Destructive Biomass Estimation from Ter- restrial LiDAR}

The first study of this project will be focused primarily on the development of an algorithm that will allow for biomass estimates of individual trees from terrestrial LiDAR data. Discrepancies in global estimates of forest biomass originate from spatial variations in allometry and wood density. Since wood density is not possible to measure with terrestrial LiDAR, improvements to allometry will be the most promising avenue of development.

Several investigators have approached biomass estimation with terrestrial LiDAR using voxelization, pixel counting, or cylinder fitting. Voxelization approaches generally overestimate tree biomass because the external voxels extend past the boundary of the outside of the tree (Bienert et al., 2014). Pixel counting methods are essentially a simplified form of voxelization, but do not include a 
stem-modeling step. This approach works well for small individuals (Seidel et al., 2011), but applications to larger trees would likely be unsuccessful, as the interior space, representing the bulk biomass of the tree, is unaccounted for. Cylinder fitting approaches are extremely promising (Calders et al., 2015b) and represent the best current options for TLS stem modeling, but generalization using cylinders is not always the most accurate representation of tree form errors often occur with non-cylindrical tree shapes like buttress roots or multiple trunks. Finally, of the existing algorithms created to estimate tree volume or biomass from terrestrial LiDAR data, very few validate their methods with destructive samples.

In Chapter 2, the primary question is: Can terrestrial LiDAR be used to estimate biomass at the individual tree scale? This question will be addressed with an alternative approach to stem modeling that accounts for external tree form and accurately estimates biomass. The algorithm will be validated with destructive measurements by the component method trunk, branches, and foliage will be measured individually in order to assess the contribution of each component to the total error of the estimate.

\section{Chapter 3: Implications of Applying Non-Destructive Local Allometry}

The primary focus of this chapter will be the creation of several local non-destructive species-specific allometric equations for the dominant canopy species in the forest. The largest of these trees can contain nearly half of the total forest biomass, yet few relationships include information of these individuals, suggesting the need for targeted improvement of their allometry. Moreover, of those studies that have used terrestrial LiDAR to estimate biomass none have evaluated the implications of application of these new allometric relationships. By applying alternative allometric equations we can evaluate changes in biomass magnitude and uncertainty.

In Chapter 3, the primary question is: How does locally derived terrestrial LiDAR allometry influence estimates of biomass compared to the more commonly used allometric relationships? This question will be addressed by three-dimensionally modeling hundreds of trees, creating allometric equations, and comparing biomass estimates and uncertainty directly to national scale equations. 


\section{Chapter 4: Improved Biomass Calibration with Terrestrial LiDAR}

While the previous chapter provides a detailed local application of terrestrial LiDAR, Chapter 4 will concentrate on the landscape applications of this technology. Terrestrial LiDAR is unique in that it provides a comparable type of data to airborne LiDAR (e.g. a point cloud format), but contains far more detailed information about the structural attributes of the forest. However, many of the methods of estimating biomass from terrestrial LiDAR are in the development stages and no current studies have evaluated how plot level estimates of biomass can be extrapolated to the landscape with other remote sensing platforms. Since plot level measurements are used to train the algorithms that ultimately estimate biomass on the landscape, changes in these values may result in changes in landscape-scale models of biomass. In the case of airborne LiDAR data - relying on the structure of the point cloud or canopy height to estimate biomass - the significant predictor variable may change. Plot level changes from TLS estimates will also likely change larger scale estimates, potentially shifting the distribution across the landscape.

In Chapter 4, the primary question is: Can terrestrial LiDAR directly reduce uncertainty in sensor calibration, validation, and biomass mapping through plotlevel 3D modeling and non-destructive allometric equations? This question will be addressed by calibrating LiDAR biomass estimates with terrestrial LiDAR-derived biomass estimates and national-scale allometry. Changes in biomass magnitude and uncertainty will be quantified in order assess TLS as a means for reducing uncertainty in biomass mapping in the sensor calibration stage. 


\section{References}

Andersen, H. E., McGaughey, R. J., \& Reutebuch, S. E. 2005. Estimating forest canopy fuel parameters using LIDAR data. Remote Sensing of Environment, 94(4), 441-449.

Aschoff, T., \& Spiecker, H. 2004. Algorithms for the automatic detection of trees in laser scanner data. International Archives of Photogrammetry, Remote Sensing and Spatial Information Sciences, 36(Part 8), W2.

Asner, Gregory P. 2009. Tropical forest carbon assessment: integrating satellite and airborne mapping approaches. Environmental Research Letters, 4(3), 034009 .

Asner, Gregory P., Mascaro, Joseph, Muller-Landau, Helene C., Vieilledent, Ghislain, Vaudry, Romuald, Rasamoelina, Maminiaina, Hall, Jefferson S., \& van Breugel, Michiel. 2012. A universal airborne LiDAR approach for tropical forest carbon mapping. Oecologia, 168(4), 1147-1160.

Asner, Gregory P., Knapp, David E., Martin, Roberta E., Tupayachi, Raul, Anderson, Christopher B., Mascaro, Joseph, Sinca, Felipe, Chadwick, K. Dana, Higgins, Mark, Farfan, William, Llactayo, William, \& Silman, Miles R. 2014. Targeted carbon conservation at national scales with high-resolution monitoring. Proceedings of the National Academy of Sciences, 111(47), E5016-E5022.

Astrup, Rasmus, Ducey, Mark J., Granhus, Aksel, Ritter, Tim, \& von Lupke, Nikolas. 2014. Approaches for estimating stand-level volume using terrestrial laser scanning in a single-scan mode. Canadian Journal of Forest ResearchRevue Canadienne De Recherche Forestiere, 44(6), 666-676.

Baccini, A., Friedl, M., Woodcock, C., \& Warbinghton, R. 2004. Forest biomass estimation over regional scales using multisource data. Geophysical Research Letters, 31.

Baccini, A., Goetz, SJ, Walker, WS, Laporte, NT, Sun, M, Sulla-Menashe, D, Hackler, J, Beck, PSA, Dubayah, R, Friedl, MA, \& others. 2012. Estimated 
carbon dioxide emissions from tropical deforestation improved by carbon-density maps. Nature Climate Change, 2(3), 182-185.

Bamler, Richard, \& Hartl, Philipp. 1998. Synthetic aperture radar interferometry. Inverse Problems, 14(4), R1.

Barnola, J. M., Anklin, M., Porcheron, J., Raynaud, D., Schwander, J., \& Stauffer, B. 1995. $\mathrm{CO}_{2}$ evolution during the last millennium as recorded by Antarctic and Greenland ice. Tellus Series B Chemical and Physical Meteorology B, 47(Feb.), 264.

Bienert, A, Maas, H.-G., \& Scheller, S. 2006a (Feb.). Analysis of the information content of terrestrial laserscanner point clouds for the automatic determination of forest inventory parameters. In: ISPRS WG VIII/11 \& EARSeL joint Conference.

Bienert, A., Scheller, S., Keane, E., Mullooly, G., \& Mohan, F. 2006b. Application of terrestrial laser scanners for the determination of forest inventory parameters. International Archives of Photogrammetry, Remote Sensing and Spatial Information Sciences, 36(Part 5).

Bienert, A., Scheller, S., Keane, E., Mohan, F., \& Nugent, C. 2007. Tree detection and diameter estimations by analysis of forest terrestrial laserscanner point clouds. Pages 50-55 of: ISPRS workshop on laser scanning, vol. 2007. Citeseer.

Bienert, A., Hess, C., Maas, H.-G., \& von Oheimb, G. 2014. A voxel-based technique to estimate the volume of trees from terrestrial laser scanner data. ISPRS - International Archives of the Photogrammetry, Remote Sensing and Spatial Information Sciences, XL-5(June), 101-106.

Box, Elgene O. 1988. Estimating the Seasonal Carbon Source-Sink Geography of a Natural, Steady-State Terrestrial Biosphere. Journal of Applied Meteorology, 27(10), 1109-1124.

Brolly, Gabor, Kiraly, Geza, \& others. 2009. Algorithms for stem mapping by means of terrestrial laser scanning. Acta Silvatica et Lignaria Hungarica, 5, 119-130.

Brown, S. 1997. Estimating biomass and biomass change of tropical forests. A primer.

Brown, S. 2002. Measuring carbon in forests: current status and future challenges. Environmental Pollution, 116(3), 363 - 372. 
Brown, S., Schroeder, P., \& Kern, J. S. 1999. Spatial distribution of biomass in forests of the eastern USA. Forest Ecology and Management, 123(1), 81-90.

Calders, Kim, Armston, John, Newnham, Glenn, Herold, Martin, \& Goodwin, Nicholas. 2014. Implications of sensor configuration and topography on vertical plant profiles derived from terrestrial LiDAR. Agricultural and Forest Meteorology, 194(Aug.), 104-117.

Calders, Kim, Schenkels, Tom, Bartholomeus, Harm, Armston, John, Verbesselt, Jan, \& Herold, Martin. 2015a. Monitoring spring phenology with high temporal resolution terrestrial LiDAR measurements. Agricultural and Forest Meteorology, 203(Apr.), 158-168.

Calders, Kim, Newnham, Glenn, Burt, Andrew, Murphy, Simon, Raumonen, Pasi, Herold, Martin, Culvenor, Darius, Avitabile, Valerio, Disney, Mathias, Armston, John, \& Kaasalainen, Mikko. 2015b. Nondestructive estimates of above-ground biomass using terrestrial laser scanning. Methods in Ecology and Evolution, 6(2), 198-208.

Calders, Kim, Burt, Andrew, Origo, Niall, \& Lewis, Philip. 2016 (July). Large-area virtual forests from terrestrial laser scanning data.

Canadell, Josep G., Le Qur, Corinne, Raupach, Michael R., Field, Christopher B., Buitenhuis, Erik T., Ciais, Philippe, Conway, Thomas J., Gillett, Nathan P., Houghton, R. A., \& Marland, Gregg. 2007. Contributions to accelerating atmospheric CO2 growth from economic activity, carbon intensity, and efficiency of natural sinks. Proceedings of the national academy of sciences, 104(47), 1886618870.

Chasmer, L., Hopkinson, C., \& Treitz, P. 2004. Assessing the three-dimensional frequency distribution of airborne and ground-based lidar data for red pine and mixed deciduous forest plots. Int Arch Photogramm Remote Sens Spat Inf Sci, 36, $8 \mathrm{~W}$.

Chave, J., Andalo, C., Brown, S., Cairns, M. A., Chambers, J. Q., Eamus, D., Flster, H., Fromard, F., Higuchi, N., Kira, T., Lescure, J.-P., Nelson, B. W., Ogawa, H., Puig, H., Rira, B., \& Yamakura, T. 2005. Tree allometry and improved estimation of carbon stocks and balance in tropical forests. Oecologia, 145(1), 87-99.

Chen, J.M., Black, T.A., \& Adams, R.S. 1991. Evaluation of Hemispherical Photography for Determining Plant-Area Index and Geometry of a Forest Stand. Agricultural and Forest Meteorology, 56(1-2), 129-143. 
Chmielewski, Leszek J., Bator, Marcin, Zasada, Michal, Sterenczak, Krzysztof, \& Strzelinski, Pawel. 2010. Fuzzy Hough Transform-Based Methods for Extraction and Measurements of Single Trees in Large-Volume 3D Terrestrial LIDAR Data. Pages 265-274 of: Bolc, L., Tadeusiewicz, R., Chmielewski, L. J., \& Wojciechowski, K. (eds), Computer Vision and Graphics, Pt I, vol. 6374.

Chojnacky, D. C., Heath, L. S., \& Jenkins, Jennifer C. 2014. Updated generalized biomass equations for North American tree species. Forestry, 87(1), 129-151.

Conway, Thomas J., Tans, P., Waterman, L. S., Thoning, K. W., Masarie, K. A., \& Gammon, R. H. 1988. Atmospheric carbon dioxide measurements in the remote global troposphere, 19811984. Tellus B, 40B(2), 81-115.

Côté, Jean-Franois, Widlowski, Jean-Luc, Fournier, Richard A., \& Verstraete, Michel M. 2009. The structural and radiative consistency of three-dimensional tree reconstructions from terrestrial lidar. Remote Sensing of Environment, 113(5), 1067-1081.

Côté, Jean-Franois, Fournier, Richard A., \& Egli, Richard. 2011. An architectural model of trees to estimate forest structural attributes using terrestrial LiDAR. Environmental Modelling \& Software, 26(6), 761-777.

Côté, Jean-Franois, Fournier, Richard A., Frazer, Gordon W., \& Olaf Niemann, K. 2012. A fine-scale architectural model of trees to enhance LiDAR-derived measurements of forest canopy structure. Agricultural and Forest Meteorology, 166-167(Dec.), 72-85.

Danson, F. Mark, Hetherington, David, Morsdorf, Felix, Koetz, Benjamin, \& Allgower, Britta. 2007. Forest Canopy Gap Fraction From Terrestrial Laser Scanning. IEEE Geoscience and Remote Sensing Letters, 4(1), 157-160.

Dassot, Mathieu, Colin, Aurlie, Santenoise, Philippe, Fournier, Meriem, \& Constant, Thiry. 2012. Terrestrial laser scanning for measuring the solid wood volume, including branches, of adult standing trees in the forest environment. Computers and Electronics in Agriculture, 89(Nov.), 86-93.

Delcourt, Hazel R, Delcourt, Paul A, \& Webb, Thompson. 1982. Dynamic plant ecology: the spectrum of vegetational change in space and time. Quaternary Science Reviews, 1(3), 153-175.

Ducey, Mark J., \& Astrup, Rasmus. 2013. Adjusting for nondetection in forest inventories derived from terrestrial laser scanning. Canadian Journal of Remote Sensing, 39(5), 410-425. 
Fan, S., Gloor, M., Mahlman, J., Pacala, S., Sarmiento, J., Takahashi, T., \& Tans, P. 1998. A Large Terrestrial Carbon Sink in North America Implied by Atmospheric and Oceanic Carbon Dioxide Data and Models. Science, 282(5388), 442-446.

Feliciano, Emanuelle A., Wdowinski, Shimon, \& Potts, Matthew D. 2014. Assessing Mangrove Above-Ground Biomass and Structure using Terrestrial Laser Scanning: A Case Study in the Everglades National Park. Wetlands, 34(5), 955-968.

Garcia, Mariano, Riano, David, Chuvieco, Emilio, \& Danson, F. Mark. 2010. Estimating biomass carbon stocks for a Mediterranean forest in central Spain using LiDAR height and intensity data. Remote Sensing of Environment, 114(4), 816-830.

Ghuneim, A. G. 2009. Contour Tracing.

Greenberg, Jonathan Asher, Dobrowski, Solomon Z., \& Ustin, Susan L. 2005. Shadow allometry: Estimating tree structural parameters using hyperspatial image analysis. Remote Sensing of Environment, 97(1), 15 - 25.

Hackenberg, Jan, Morhart, Christopher, Sheppard, Jonathan, Spiecker, Heinrich, \& Disney, Mathias. 2014. Highly Accurate Tree Models Derived from Terrestrial Laser Scan Data: A Method Description. Forests, 5(5), 1069-1105.

Hackenberg, Jan, Spiecker, Heinrich, Calders, Kim, Disney, Mathias, \& Raumonen, Pasi. 2015. SimpleTree-An Efficient Open Source Tool to Build Tree Models from TLS Clouds. Forests, 6(11), 4245-4294.

Hall, Forrest G., Bergen, Kathleen, Blair, James B., Dubayah, Ralph, Houghton, Richard, Hurtt, George, Kellndorfer, Josef, Lefsky, Michael, Ranson, Jon, Saatchi, Sasan, Shugart, H. H., \& Wickland, Diane. 2011. Characterizing 3D vegetation structure from space: Mission requirements. Remote Sensing of Environment, 115(11), 2753 - 2775 .

Hall, R. J., Skakun, R. S., Arsenault, E. J., \& Case, B. S. 2006. Modeling forest stand structure attributes using Landsat ETM+ data: Application to mapping of aboveground biomass and stand volume. Forest Ecology and Management, 225(1-3), 378-390.

Henning, Jason G., \& Radtke, Philip J. 2006a. Detailed stem measurements of standing trees from ground-based scanning lidar. Forest Science, 52(1), 67-80. 
Henning, Jason G., \& Radtke, Philip J. 2006b. Ground-based laser imaging for assessing three-dimensional forest canopy structure. Photogrammetric Engineering E Remote Sensing, 72(12), 1349-1358.

Hildebrandt, Rdiger, \& Iost, Andr. 2012. From points to numbers: a databasedriven approach to convert terrestrial LiDAR point clouds to tree volumes. European Journal of Forest Research, 131(6), 1857-1867.

Hinks, Tommy, Carr, Hamish, Truong-Hong, Linh, \& Laefer, Debra F. 2012. Point Cloud Data Conversion into Solid Models via Point-Based Voxelization. Journal of Surveying Engineering, 139(2), 72-83.

Hopkinson, Chris, Chasmer, Laura, Young-Pow, Colin, \& Treitz, Paul. 2004. Assessing forest metrics with a ground-based scanning lidar. Canadian Journal of Forest Research, 34(3), 573-583.

Hosoi, Fumiki, \& Omasa, Kenji. 2006. Voxel-Based 3-D Modeling of Individual Trees for Estimating Leaf Area Density Using High-Resolution Portable Scanning Lidar. IEEE Transactions on Geoscience and Remote Sensing, 44(12), $3610-3618$.

Hosoi, Fumiki, \& Omasa, Kenji. 2007. Factors contributing to accuracy in the estimation of the woody canopy leaf area density profile using 3D portable lidar imaging. Journal of Experimental Botany, 58(12), 3463-3473.

Hosoi, Fumiki, \& Omasa, Kenji. 2009a. Detecting seasonal change of broadleaved woody canopy leaf area density profile using 3D portable LIDAR imaging. FUNCTIONAL PLANT BIOLOGY, 36(10-11), 998-1005.

Hosoi, Fumiki, \& Omasa, Kenji. 2009b. Estimating vertical plant area density profile and growth parameters of a wheat canopy at different growth stages using three-dimensional portable lidar imaging. ISPRS Journal of Photogrammetry and Remote Sensing, 64(2), 151-158.

Hosoi, Fumiki, Yoshimi, K., Shimizu, Y., \& Omasa, K. 2005. 3-D measurement of trees using a portable scanning Lidar. PHYTON-ANNALES REI BOTANICAE, 45(4, SI), 497-500.

Hosoi, Fumiki, Nakai, Yohei, \& Omasa, Kenji. 2010. Estimation and Error Analysis of Woody Canopy Leaf Area Density Profiles Using 3-D Airborne and GroundBased Scanning Lidar Remote-Sensing Techniques. IEEE Transactions on Geoscience and Remote Sensing, 48(5), 2215-2223. 
Hosoi, Fumiki, Nakai, Yohei, \& Omasa, Kenji. 2013a. 3-D voxel-based solid modeling of a broad-leaved tree for accurate volume estimation using portable scanning lidar. ISPRS Journal of Photogrammetry and Remote Sensing, 82(Aug.), 41-48.

Hosoi, Fumiki, Nakai, Yohei, \& Omasa, K. 2013b. Voxel tree modeling for estimating leaf area density and woody material volume using 3-D LIDAR data. ISPRS Annals of Photogrammetry, Remote Sensing and Spatial Information Sciences, II-5/W2(Oct.), 115-120.

Houghton, R. A. 2003. Revised estimates of the annual net flux of carbon to the atmosphere from changes in land use and land management 18502000. Tellus $B, 55(2), 378-390$.

Houghton, R. A. 2005. Aboveground forest biomass and the global carbon balance. Global Change Biology, 11.

Houghton, R. A., Hall, Forrest, \& Goetz, Scott J. 2009. Importance of biomass in the global carbon cycle. Journal of Geophysical Research-Biogeosciences, 114(Sept.), G00E03.

IPCC. 2006. 2006 IPCC Guidelines for National Greenhouse Gas Inventories.

Jaakkola, Anttoni, Hyypp, Juha, Kukko, Antero, Yu, Xiaowei, Kaartinen, Harri, Lehtomki, Matti, \& Lin, Yi. 2010. A low-cost multi-sensoral mobile mapping system and its feasibility for tree measurements. ISPRS Journal of Photogrammetry and Remote Sensing, 65(6), 514-522.

Jenkins, J. C., Chojnacky, David C., Heath, Linda S., \& Birdsey, Richard A. 2003. National-scale biomass estimators for United States tree species. Forest Science, 49(1), 12-35.

Jenkins, Jennifer C., Birdsey, Richard A., \& Pan, Yude. 2001. Biomass and Npp Estimation for the Mid-Atlantic Region (usa) Using Plot-Level Forest Inventory Data. Ecological Applications, 11(4), 1174-1193.

Jupp, David L. B., Lovell, Jennifer L, Marine, CSIRO, \& Research, Atmospheric. 2007. Airborne and ground-based LIDAR systems for forest measurement background and principles. Canberra, A.C.T. : CSIRO Marine and Atmospheric Research.

Jupp, David L. B., Culvenor, D.S., Lovell, J.L., Newnham, G.J., Strahler, A.H., \& Woodcock, C.E. 2008. Estimating forest LAI profiles and structural parameters using a ground-based laser called Echidna. Tree Physiology, 29(2), 171-181. 
Keeling, Charles D. 1979. The Suess effect: 13Carbon-14Carbon interrelations. Environment International, 2(4), 229 - 300.

Keeling CD, Whorf TP, Wahlen M, \& Vanderplicht J. 1995. Interannual Extremes in the Rate of Rise of Atmospheric Carbon-Dioxide Since 1980. Nature, 375(6533), 666-670.

Koch, Barbara. 2010. Status and future of laser scanning, synthetic aperture radar and hyperspectral remote sensing data for forest biomass assessment. $\{$ ISPRS Journal of Photogrammetry and Remote Sensing, 65(6), 581 - 590.

Koch, G. W., Sillett, S. C., Jennings, G. M., \& Davis, S. D. 2004. The limits to tree height. Nature, 428(6985), 851-854.

Le Toan, T., Beaudoin, A., Riom, J., \& Guyon, D. 1992. Relating forest biomass to SAR data. IEEE Transactions on Geoscience and Remote Sensing, 30(2), 403-411.

Le Toan, T., Quegan, S., Davidson, M. W. J., Balzter, H., Paillou, P., Papathanassiou, K., Plummer, S., Rocca, F., Saatchi, S., Shugart, H., \& Ulander, L. 2011. The BIOMASS mission: Mapping global forest biomass to better understand the terrestrial carbon cycle. Remote Sensing of Environment, 115(11), 2850-2860.

Lefsky, Michael, \& McHale, Melissa. 2008. Volume estimates of trees with complex architecture from terrestrial laser scanning. Journal of Applied Remote Sensing, 2, 023521 .

Lefsky, Michael A., Cohen, W. B., Acker, S. A., Parker, G. G., Spies, T. A., \& Harding, D. 1999. Lidar remote sensing of the canopy structure and biophysical properties of Douglas-fir western hemlock forests. Remote Sensing of Environment, 70(3), 339-361.

Lefsky, Michael A., Cohen, Warren B., Parker, Geoffrey G., \& Harding, David J. 2002. Lidar Remote Sensing for Ecosystem Studies. BioScience, 52(1), 19.

Li, X., \& Strahler, A. H. 1985. Geometric-Optical Modeling of a Conifer Forest Canopy. IEEE Transactions on Geoscience and Remote Sensing, GE-23(5), $705-721$.

Liang, X., Litkey, P., Hyyppa, J., Kukko, A., Kaartinen, H., \& Holopainen, M. 2008. Plot-level trunk detection and reconstruction using one-scan-mode terrestrial laser scanning data. Pages 136-140 of: 2008 INTERNATIONAL WORKSHOP ON EARTH OBSERVATION AND REMOTE SENSING APPLICATIONS. 345 E 47TH ST, NEW YORK, NY 10017 USA: IEEE. 
Liang, Xinlian, \& Hyyppa, Juha. 2013. Automatic Stem Mapping by Merging Several Terrestrial Laser Scans at the Feature and Decision Levels. Sensors, 13(2), 1614-1634.

Liang, Xinlian, Kankare, Ville, Yu, Xiaowei, Hyyppa, Juha, \& Holopainen, Markus. 2014. Automated Stem Curve Measurement Using Terrestrial Laser Scanning. Ieee Transactions on Geoscience and Remote Sensing, 52(3), 17391748 .

Lovell, J. L., Jupp, D. L. B., Newnham, G. J., \& Culvenor, D. S. 2011. Measuring tree stem diameters using intensity profiles from ground-based scanning lidar from a fixed viewpoint. Isprs Journal of Photogrammetry and Remote Sensing, 66(1), 46-55.

Maas, H.G., Bienert, A., Scheller, S., \& Keane, E. 2008. Automatic forest inventory parameter determination from terrestrial laser scanner data. International Journal of Remote Sensing, 29(5), 1579-1593.

MacArthur, Robert H., \& Horn, Henry S. 1969. Foliage Profile by Vertical Measurements. Ecology, 50(5), 802.

Mann, M. E., Bradley, R. S., \& Hughes, M. K. 1998. Global-scale temperature patterns and climate forcing over the past six centuries. Nature, 392(6678), $779-787$.

McDaniel, Matthew W., Nishihata, Takayuki, Brooks, Christopher A., Salesses, Phil, \& Iagnemma, Karl. 2012. Terrain classification and identification of tree stems using ground-based LiDAR. JOURNAL OF FIELD ROBOTICS, 29(6), 891-910.

Meagher, D.J.R. 1980. Octree Encoding: a New Technique for the Representation, Manipulation and Display of Arbitrary 3-D Objects by Computer.

Mitchard, Edward T. A., Saatchi, S. S., White, L. J. T., Abernethy, K. A., Jeffery, K. J., Lewis, S. L., Collins, M., Lefsky, M. A., Leal, M. E., Woodhouse, I. H., \& Meir, P. 2012. Mapping tropical forest biomass with radar and spaceborne LiDAR in Lope National Park, Gabon: overcoming problems of high biomass and persistent cloud. Biogeosciences, 9(1), 179-191.

Mitchard, Edward T. A., Feldpausch, Ted R., Brienen, Roel J. W., LopezGonzalez, Gabriela, Monteagudo, Abel, Baker, Timothy R., Lewis, Simon L., Lloyd, Jon, Quesada, Carlos A., Gloor, Manuel, ter Steege, Hans, Meir, Patrick, Alvarez, Esteban, Araujo-Murakami, Alejandro, Aragao, Luiz E. O. C., Arroyo, Luzmila, Aymard, Gerardo, Banki, Olaf, Bonal, Damien, Brown, Sandra, 
Brown, Foster I., Ceron, Carlos E., Chama Moscoso, Victor, Chave, Jerome, Comiskey, James A., Cornejo, Fernando, Corrales Medina, Massiel, Da Costa, Lola, Costa, Flavia R. C., Di Fiore, Anthony, Domingues, Tomas F., Erwin, Terry L., Frederickson, Todd, Higuchi, Niro, Honorio Coronado, Euridice N., Killeen, Tim J., Laurance, William F., Levis, Carolina, Magnusson, William E., Marimon, Beatriz S., Marimon Junior, Ben Hur, Mendoza Polo, Irina, Mishra, Piyush, Nascimento, Marcelo T., Neill, David, Nunez Vargas, Mario P., Palacios, Walter A., Parada, Alexander, Pardo Molina, Guido, Pena-Claros, Marielos, Pitman, Nigel, Peres, Carlos A., Poorter, Lourens, Prieto, Adriana, RamirezAngulo, Hirma, Restrepo Correa, Zorayda, Roopsind, Anand, Roucoux, Katherine H., Rudas, Agustin, Salomao, Rafael P., Schietti, Juliana, Silveira, Marcos, de Souza, Priscila F., Steininger, Marc K., Stropp, Juliana, Terborgh, John, Thomas, Raquel, Toledo, Marisol, Torres-Lezama, Armando, van Andel, Tinde R., van der Heijden, Geertje M. F., Vieira, Ima C. G., Vieira, Simone, Vilanova-Torre, Emilio, Vos, Vincent A., Wang, Ophelia, Zartman, Charles E., Malhi, Yadvinder, \& Phillips, Oliver L. 2014. Markedly divergent estimates of Amazon forest carbon density from ground plots and satellites. Global Ecology and Biogeography, 23(8), 935-946.

Moskal, L. Monika, Erdody, Todd, Kato, Akira, Richardson, Jeffery, Zheng, Guang, \& Briggs, David. 2009. LiDAR applications in precision forestry. In: Proceedings of the 2009 Silvilaser Conference.

Myneni, R. B., Hoffman, S., Knyazikhin, Y., Privette, J. L., Glassy, J., Tian, Y., Wang, Y., Song, X., Zhang, Y., Smith, G. R., Lotsch, A., Friedl, M., Morisette, J. T., Votava, P., Nemani, R. R., \& Running, S. W. 2002. Global products of vegetation leaf area and fraction absorbed $\{$ PAR $\}$ from year one of $\{$ MODIS data. Remote Sensing of Environment, 83(12), 214 - 231.

Naesset, E. 2002. Predicting forest stand characteristics with airborne scanning laser using a practical two-stage procedure and field data. Remote Sensing of Environment, 80(1), 88-99.

Nelson, R., Keller, C., \& Ratnaswamy, M. 2005. Locating and estimating the extent of Delmarva fox squirrel habitat using an airborne LiDAR profiler. Remote Sensing of Environment, 96(3-4), 292-301.

Nogueira, Euler Melo, Nelson, Bruce Walker, Fearnside, Philip Martin, Frana, Mabiane Batista, \& Oliveira, tila Cristina Alves de. 2008. Tree height in Brazil's arc of deforestation: Shorter trees in south and southwest Amazonia imply lower biomass. Forest Ecology and Management, 255(7), 2963 - 2972. 
Olagoke, Adewole, Proisy, Christophe, Fret, Jean-Baptiste, Blanchard, Elodie, Fromard, Franois, Mehlig, Ulf, de Menezes, Moirah Machado, dos Santos, Valdenira Ferreira, \& Berger, Uta. 2016. Extended biomass allometric equations for large mangrove trees from terrestrial LiDAR data. Trees, 30(3), 935-947.

Omasa, Kenji, Hosoi, Fumiki, \& Konishi, A. 2006. 3D lidar imaging for detecting and understanding plant responses and canopy structure. Journal of Experimental Botany, 58(4), 881-898.

Omasa, Kenji, Hosoi, Fumiki, Uenishi, T. M., Shimizu, Y., \& Akiyama, Y. 2008. Three-Dimensional Modeling of an Urban Park and Trees by Combined Airborne and Portable On-Ground Scanning LIDAR Remote Sensing. Environmental Modeling \& Assessment, 13(4), 473-481.

Palace, Michael, Keller, Michael, Asner, Gregory P., Hagen, Stephen, \& Braswell, Bobby. 2008. Amazon Forest Structure from IKONOS Satellite Data and the Automated Characterization of Forest Canopy Properties. Biotropica, 40(2), $141-150$.

Pan, Yude, Birdsey, Richard A., Fang, Jingyun, Houghton, Richard, Kauppi, Pekka E., Kurz, Werner A., Phillips, Oliver L., Shvidenko, Anatoly, Lewis, Simon L., Canadell, Josep G., Ciais, Philippe, Jackson, Robert B., Pacala, Stephen W., McGuire, A. David, Piao, Shilong, Rautiainen, Aapo, Sitch, Stephen, \& Hayes, Daniel. 2011. A Large and Persistent Carbon Sink in the World's Forests. Science, 333(6045), 988-993.

Pfeifer, Norbert, \& Winterhalder, Daniel. 2004. Modelling of tree cross sections from terrestrial laser scanning data with free-form curves. Int. Arch. Photogramm. Remote Sens. Spat. Inf. Sci, 36(8/W2), 76-81.

Pfeifer, Norbert, Gorte, Ben, \& Winterhalder, Daniel. 2004. Automatic reconstruction of single trees from terrestrial laser scanner data. Pages 114-119 of: Proceedings of 20th ISPRS Congress.

Pimont, Francois, Dupuy, Jean-Luc, Rigolot, Eric, Prat, Vincent, \& Piboule, Alexandre. 2015. Estimating Leaf Bulk Density Distribution in a Tree Canopy Using Terrestrial LiDAR and a Straightforward Calibration Procedure. Remote Sensing, 7(6), 7995-8018.

Popescu, Sorin C., Wynne, Randolph H., \& Scrivani, John A. 2004. Fusion of small-footprint lidar and multispectral data to estimate plot-level volume and biomass in deciduous and pine forests in Virginia, USA. Forest Science, 50(4), $551-565$. 
Raumonen, Pasi, Kaasalainen, Mikko, kerblom, Markku, Kaasalainen, Sanna, Kaartinen, Harri, Vastaranta, Mikko, Holopainen, Markus, Disney, Mathias, \& Lewis, Philip. 2013. Fast Automatic Precision Tree Models from Terrestrial Laser Scanner Data. Remote Sensing, 5(2), 491-520.

Rauste, Yrjo. 2005. Multi-temporal \{JERS $\}$ SAR $\}$ data in boreal forest biomass mapping. Remote Sensing of Environment, 97(2), 263 - 275.

Rutzinger, M., Pratihast, A. K., Oude Elberink, S., \& Vosselman, George. 2010. Detection and modelling of 3D trees from mobile laser scanning data. Int. Arch. Photogramm. Remote Sens. Spat. Inf. Sci, 38, 520-525.

Saatchi, Sassan, Houghton, R. A., Alvala, R. C. Dos Santos, Soares, J. V., \& Yu, Y. 2007. Distribution of aboveground live biomass in the Amazon basin. Global Change Biology, 13(4), 816-837.

Saatchi, Sassan, Harris, Nancy L., Brown, Sandra, Lefsky, Michael, Mitchard, Edward T. A., Salas, William, Zutta, Brian R., Buermann, Wolfgang, Lewis, Simon L., Hagen, Stephen, Petrova, Silvia, White, Lee, Silman, Miles, \& Morel, Alexandra. 2011. Benchmark map of forest carbon stocks in tropical regions across three continents. Proceedings of the National Academy of Sciences of the United States of America, 108(24), 9899-9904.

Schimel, David S. 1995. Terrestrial ecosystems and the carbon cycle. Global Change Biology, 1(1), 77-91.

Schlesinger, William H., \& Bernhardt, Emily S. 2013a. Chapter 11 - The Global Carbon Cycle. Pages 419 - 444 of: Schlesinger, William H., \& Bernhardt, Emily S. (eds), Biogeochemistry (Third Edition), third edition edn. Boston: Academic Press.

Schlesinger, William H., \& Bernhardt, Emily S. 2013b. Chapter 3 - The Atmosphere. Pages 49 - 91 of: Schlesinger, William H., \& Bernhardt, Emily S. (eds), Biogeochemistry (Third Edition), third edition edn. Boston: Academic Press.

Schnabel, Ruwen, Wahl, Roland, \& Klein, Reinhard. 2007. Efficient RANSAC for Point-Cloud Shape Detection. Pages 214-226 of: Computer graphics forum, vol. 26. Wiley Online Library.

Seidel, Dominik, Beyer, Friderike, Hertel, Dietrich, Fleck, Stefan, \& Leuschner, Christoph. 2011. 3D-laser scanning: A non-destructive method for studying above- ground biomass and growth of juvenile trees. Agricultural and Forest Meteorology, 151(10), 1305 - 1311. 
Seidel, Dominik, Fleck, Stefan, \& Leuschner, Christoph. 2012. Analyzing forest canopies with ground-based laser scanning: A comparison with hemispherical photography. Agricultural and Forest Meteorology, 154-155(Mar.), 1-8.

Shugart, Herman H., Saatchi, Sassan, \& Hall, F. G. 2010. Importance of structure and its measurement in quantifying function of forest ecosystems. Journal of Geophysical Research, 115(Nov.).

Shugart, Herman H, Asner, Gregory P, Fischer, Rico, Huth, Andreas, Knapp, Nikolai, Le Toan, Thuy, \& Shuman, Jacquelyn K. 2015. Computer and remotesensing infrastructure to enhance large-scale testing of individual-based forest models. Frontiers in Ecology and the Environment, 13(9), 503-511.

Simard, Marc, Pinto, Naiara, Fisher, Joshua B., \& Baccini, Alessandro. 2011. Mapping forest canopy height globally with spaceborne lidar. Journal of Geophysical Research-Biogeosciences, 116(Nov.), G04021.

Smith, Aaron, Astrup, Rasmus, Raumonen, Pasi, Liski, Jari, Krooks, Anssi, Kaasalainen, Sanna, Akerblom, Markku, \& Kaasalainen, Mikko. 2014. Tree Root System Characterization and Volume Estimation by Terrestrial Laser Scanning and Quantitative Structure Modeling. Forests, 5(12), 3274-3294.

Soenen, Scott A., Peddle, Derek R., Hall, Ronald J., Coburn, Craig A., \& Hall, Forrest G. 2010. Estimating aboveground forest biomass from canopy reflectance model inversion in mountainous terrain. Remote Sensing of Environment, 114(7), $1325-1337$.

Tansey, K., Selmes, N., Anstee, A., Tate, N. J., \& Denniss, A. 2009. Estimating tree and stand variables in a Corsican Pine woodland from terrestrial laser scanner data. International Journal of Remote Sensing, 30(19), 5195-5209.

TerMikaelian, M. T., \& Korzukhin, M. D. 1997. Biomass equations for sixty-five North American tree species. Forest Ecology and Management, 97(1), 1-24.

Wegmuller, U., \& Werner, C. L. 1995. SAR interferometric signatures of forest. IEEE Transactions on Geoscience and Remote Sensing, 33(5), 1153-1161.

Xia, Shaobo, Wang, Cheng, Pan, Feifei, Xi, Xiaohuan, Zeng, Hongcheng, \& Liu, He. 2015. Detecting Stems in Dense and Homogeneous Forest Using Single-Scan TLS. Forests, 6(11), 3923-3945.

Yang, Bisheng, Dai, Wenxia, Dong, Zhen, \& Liu, Yang. 2016. Automatic Forest Mapping at Individual Tree Levels from Terrestrial Laser Scanning Point Clouds with a Hierarchical Minimum Cut Method. REMOTE SENSING, 8(5). 
Zhang, Xiaoyang, \& Kondragunta, Shobha. 2006. Estimating forest biomass in the USA using generalized allometric models and MODIS land products. Geophysical Research Letters, 33(9), n/a-n/a.

Zhao, Kaiguang, Popescu, Sorin, \& Nelson, Ross. 2009. Lidar remote sensing of forest biomass: A scale-invariant estimation approach using airborne lasers. Remote Sensing of Environment, 113(1), 182-196. 


\section{Chapter 2}

Non-Destructive Biomass Estimation from Terrestrial LiDAR

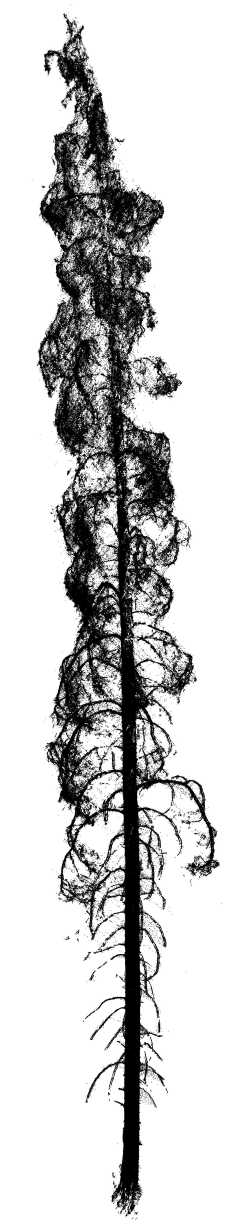




\section{Abstract}

Global estimates of forest aboveground biomass and carbon storage have major discrepancies linked to limitations in tree-level biomass estimates. Robust allometric equations can improve biomass estimates; however, destructive sampling to measure single-tree biomass is expensive, challenging, and prone to measurement error. We present a method to efficiently and non-destructively estimate single-tree biomass from terrestrial LiDAR scan data and test the approach on 21 destructively-sampled lodgepole pine (Pinus contorta) trees. The approach estimates branch and foliage volume using voxelization and estimates trunk volume using a method developed in this study called the Outer Hull Model (OHM). The OHM iteratively fits convex hulls, accurately handles noisy scan data, and fits the true shape of the trunk rather than forcing a cylindrical fit. Volume from the LiDAR scans is converted to biomass using density values from the literature and from field sampling to assess model sensitivity to density values. Whole-tree aboveground biomass estimates derived from the LiDAR scans were nearly unbiased and agreed strongly with destructive sampling data $\left(\mathrm{R}^{2}=0.98, \mathrm{RMSE}=20.4 \mathrm{~kg}\right)$. Estimation of the trunk component biomass $\left(\mathrm{R}^{2}=0.99, \mathrm{RMSE}=12.3 \mathrm{~kg}\right)$ was stronger than foliage and needle component estimates $\left(\mathrm{R}^{2}=0.54, \mathrm{RMSE}=21.4 \mathrm{~kg}\right)$. The approach presented in this study accurately and non-destructively estimated the aboveground biomass of needleleaf trees with minimal user input. The promising performance on coniferous trees advances efficient sampling of single-tree biomass. ${ }^{1}$

\footnotetext{
${ }^{1}$ This chapter is based on: Stovall, A.E.L., Vorster, A.G., Anderson, R.S., Evangelista, P.H., Shugart, H.H., 2017. Non-destructive aboveground biomass estimation of coniferous trees using terrestrial LiDAR. Remote Sensing of Environment 200(Oct.), 31-42. doi:10.1016/j.rse.2017.08.013
} 


\subsection{Introduction}

Forest biomass quantification is important for ecosystem service management and understanding terrestrial carbon dynamics (Houghton et al., 2009). However, global estimates of aboveground biomass and carbon storage have major discrepancies linked to limitations in single-tree biomass estimates (Saatchi et al., 2011; Baccini et al., 2012; Mitchard et al., 2014). Converting tree diameter and height to biomass using allometric equations is uncertain - relying on the ability of an individual equation to capture variations, not only by species and growth form, but also spatially, depending on climate, soil, nutrient availability, and intra/interspecific competition (Feldpausch et al., 2011). Uncertainty in biomass distribution would be substantially reduced by increasing the sample size for allometric equations to capture the variability in tree form across the landscape. However, collecting large sample sizes through cutting and weighing trees, or destructive sampling, is a time-consuming and expensive process that is subject to sampling bias and high uncertainty (Chave et al., 2004; Picard et al., 2012). The feasibility of destructive sampling is further limited by capital, labor, logistics and protected areas (Brown, 1997; Picard et al., 2012) and samples are often underrepresented in areas of complex topography, variable climatic conditions, cultural significance, or political restriction. These limitations are especially prevalent in mature forests, where large, difficult to sample trees drive high biomass density. Many of these challenges can be reduced or eliminated by estimating single-tree biomass non-destructively using terrestrial LiDAR (Light Detection and Ranging).

In recent years, forestry applications of terrestrial LiDAR, or terrestrial laser scanning (TLS), have been explored to automate the measurement of standard forestry inventory parameters, as well as non-destructively estimate tree-level biomass (Dassot et al., 2011; Liang et al., 2016). Terrestrial laser scanning has been used in forestry to automate tree diameter and height measurements (Hopkinson et al., 2004; Bienert et al., 2006; Henning \& Radtke, 2006; Maas et al., 2008; Lovell et al., 2011). More complex forest structure characteristics, such as vertically distributed leaf area index (LAI) and gap fraction, have been measured by applying techniques from airborne LiDAR (Lovell et al., 2003; Danson et al., 2007; Jupp et al., 2008; Yao et al., 2011; Zhao et al., 2011; Griebel et al., 2015). 
Volume is typically extracted from terrestrial LiDAR scan data using two main approaches: voxelization procedures, which convert structured point clouds to cubes (Lefsky \& McHale, 2008; Hosoi et al., 2013), and cylinder-fitting algorithms, which model the tree stem and branches with a best-fit cylindrical shape (Côté et al., 2009, 2011; Raumonen et al., 2013; Hackenberg et al., 2014; Calders et al., 2015). Volume derived from either of these methods is combined with estimated or sampled species-specific wood density to non-destructively estimate biomass. Current techniques to estimate biomass from terrestrial LiDAR data vary considerably in effectiveness, are not applicable across all tree growth forms, and few validate with destructive measurements. Performance of many methods relies on low-noise data (Hackenberg et al., 2015a), which presents a significant obstacle for automated volume extraction from noisier data collected with portable, lowercost, phase-based LiDAR scanners. Voxelization algorithms provide an excellent representation of branch complexity from the raw data, but tends to overestimate stem volume (Bienert et al., 2014). Overestimates occur because only a portion of the voxels associated with the exterior of the tree can be attributed to tree volume. Cylinder-fitting approaches are nearly fully automated and have been validated against a large number of destructive biomass measurements (Calders et al., 2015). This method can be very accurate for simple tree structures, but in dense or clumped tree canopies that have significant occlusion, this approach can result in unexpected and unrealistic volumes (Hackenberg et al., 2015b). Also, algorithms that rely on cylinders are invariably error-prone when applied to non-cylindrical tree structures (e.g. buttress roots, multiple trunks, etc.). No cylinder-fitting approaches that we are aware of include the foliage component when quantifying whole-tree biomass with validated destructive measurements.

Our objective was to develop and validate a method that efficiently estimates whole-tree and component biomass from terrestrial LiDAR data. Our approach estimates branch and foliage volume using voxelization and estimates trunk volume using a convex hull peeling method developed in this study that we term the Outer Hull Model (OHM). This method overcomes limitations present in the most common algorithms in the handling of noisy scan data, non-cylindrical shapes, and occlusion. We validated our algorithm using 14 live and 7 dead lodgepole pine (Pinus contorta) trees that were destructively sampled in the Colorado State 
Forest State Park, USA. We also use these samples to determine the error in biomass estimation attributed to per-tree density variability.

\subsection{Methods}

We destructively harvested 21 lodgepole pine trees to measure component biomass trunk, branches, and needles and estimated diameter at breast height (DBH), total height, and aboveground biomass of each component using terrestrial LiDAR. We evaluated the contribution of density-driven biomass uncertainty by applying density measured from each destructively-sampled tree to our non-destructive biomass estimation.

\subsubsection{Study Area}

This study was conducted at the Colorado State Forest State Park in northern Colorado's Medicine Bow Range, USA. The 28,732 ha forest is managed for multiple uses, including timber, recreation, grazing, water quality, education, and wildlife. Mountain pine beetles (Dendroctonus ponderosae) caused extensive lodgepole pine mortality within the park between 2005 and 2011 (USDA Forest Service, 2013) where $75 \%$ of the basal area was killed in the stand where this study was conducted. The stand is situated at 2,708 $\mathrm{m}$ above sea level on an average slope of $14 \%$ on a north-northeast aspect. Average annual precipitation and temperature in the area are $560 \mathrm{~mm}$ and $2^{\circ} \mathrm{C}$, respectively. The trees used in this study grew in an even-aged lodgepole pine stand, although some of the smaller-diameter trees we sampled were younger than the overstory pine. Stand density averaged 735 trees $\mathrm{ha}^{-1}(\min =238, \max =1366)$ and basal area averaged $26.9 \mathrm{~m}^{2} \mathrm{ha}^{-1}(\min =0.5$, $\max =50.0)$. An average of $14.7 \mathrm{~m}^{2} \mathrm{ha}^{-1}(\min =0.0, \max =43.4)$ was removed when the stand was last thinned (Vorster and Anderson, unpublished data).

\subsubsection{Destructive Sampling}

Twenty-one lodgepole pine trees were selected to represent the diameter range and typical growth form in the stand (Table 2.1). We selected both live trees $(n=14)$ 
and trees killed by mountain pine beetles approximately seven years before sampling $(n=7)$. Needles drop from trees several years after being killed by mountain pine beetles. This provided an opportunity to scan pine trees with LiDAR without occlusion by needles, similar to leaf-off scanning of deciduous trees (Dassot et al., 2011; Hosoi et al., 2013). One additional live tree was only sampled for trunk biomass $(n=22)$. We scanned and destructively sampled the trees between July and September, 2014.

\section{Field Sampling}

Each sample tree was felled on a tarpaulin to begin destructive sampling. We recorded total stem height, crown height (starting at first live branch) and height to the four-inch diameter of the main stem. The crown, the section between the first live branch and the four-inch diameter, was measured next. For each crown branch, we measured diameter and the distance from the tree base and for every third branch, we measured length. Six subsample branches from the crown were intensively sampled to determine wood and foliage mass and were then oven dried. All other crown branches were sorted as live or dead and weighed. We only had four subsample branches for one tree and five for another due to rain and a lack of crown branches below the four-inch diameter. The tree top, defined as the biomass above the four-inch diameter, was next weighed in three groups: the top main stem, live top branches, and dead top branches. Once the crown branches and tree top were stripped from the bole, we cut the bole of the tree from the base to the four-inch diameter into four-foot segments to be weighed. A disk was cut from the top of each four-foot segment to measure bole density and bark.

Several of the procedures varied for dead trees. Five branches from two dead trees, for a total of ten branches, were weighed and oven dried. The average moisture content from these ten branches was used to calculate the dry mass of dead branches on dead trees and in the crown and top of live trees. The first branch, rather than the first live branch, was considered the start of the crown for dead trees. The bark on the dead trees did not cleanly peel off the disks, so a roughly $10 \mathrm{~cm}$ portion of bark was shaved into a bag, but the rest of the bark was kept on the disks. 
Table 2.1: Summary statistics for 21 destructively-sampled lodgepole pine (Pinus contorta) trees. Trunk biomass was measured for 22 trees.

\begin{tabular}{lccccc}
\hline \hline Component & Status & Mean & St. Dev. & Min & Max \\
\hline DBH $(\mathrm{cm})$ & Both & 21.4 & 6.4 & 10.2 & 33.6 \\
& Live & 19.9 & 6.1 & 10.2 & 29.9 \\
& Dead & 24.5 & 5.4 & 16.5 & 33.6 \\
\hline Height $(\mathrm{m})$ & Both & 16.5 & 5.2 & 5.9 & 22.4 \\
& Live & 15.1 & 5.4 & 5.9 & 21.1 \\
& Dead & 19.4 & 2.8 & 14.4 & 22.4 \\
\hline Total Biomass $(\mathrm{kg})$ & Both & 178.5 & 123.9 & 13.9 & 458.0 \\
& Live & 152.3 & 113.4 & 13.9 & 354.8 \\
& Dead & 230.9 & 136.2 & 62.7 & 458.0 \\
\hline Woody Biomass $(\mathrm{kg})$ & Both & 169.6 & 122.8 & 10.8 & 458.0 \\
& Live & 139.0 & 107.7 & 10.8 & 332.9 \\
& Dead & 230.9 & 136.2 & 62.7 & 458.0 \\
\hline Trunk Biomass $(\mathrm{kg})$ & Both & 146.6 & 108.2 & 4.5 & 388.1 \\
& Live & 121.7 & 100.0 & 4.5 & 303.9 \\
& Dead & 200.1 & 112.9 & 56.3 & 388.1 \\
\hline Branch Biomass $(\mathrm{kg})$ & Both & 30.5 & 22.3 & 5.6 & 70.1 \\
& Live & 30.3 & 21.7 & 5.6 & 70.1 \\
& Dead & 30.8 & 25.3 & 6.4 & 69.9 \\
\hline Branch and Needle Biomass $(\mathrm{kg})$ & Live & 43.6 & 28.1 & 9.4 & 92.0 \\
\hline Needle Biomass $(\mathrm{kg})$ & Live & 13.3 & 6.8 & 3.1 & 27.1 \\
\hline Wood Density $\left(\mathrm{g} \mathrm{cm}{ }^{-3}\right)$ & Both & 0.39 & 0.03 & 0.33 & 0.45 \\
& Live & 0.39 & 0.03 & 0.33 & 0.45 \\
& Dead & 0.39 & 0.04 & 0.34 & 0.44 \\
\hline & & & & & \\
& & & & \\
& & & & & \\
& & &
\end{tabular}




\section{Component Calculations}

The whole-tree dry biomass and bole, branch wood, and foliage components were calculated for each tree. The disks, bark, subsampled branch wood, and subsampled foliage were dried at $105^{\circ} \mathrm{C}$ (de Miguel et al., 2014) until the sample mass changed by less than a gram over three days. The moisture content determined from oven drying was used to estimate dry biomass. We calculated the density of each tree as the average dry density of all bole disks from a tree.

To calculate trunk component biomass, the summed four-foot bole segments were separated into bole wood and bark wet mass. The average water proportions determined from the oven-dried disks and bark subsamples from each tree were then subtracted from the bole wood and bark wet mass, respectively. Bole wood and bark dry mass were summed to arrive at trunk dry mass. The branch wood and needle components were composed of biomass from the crown and the top of the tree. Crown branch length was only measured for every third branch, so we used linear regression to predict length of unmeasured branches from branch diameter $\left(\mathrm{n}=255, \mathrm{R}^{2}=0.54, \mathrm{RMSE}=0.4 \mathrm{~m}, 31 \%\right)$. We modeled wet mass of foliage and wood for each branch using linear and nonlinear models with branch diameter and length as predictor variables (Kershaw \& Maguire, 1995; Temesgen et al., 2011; Poudel et al., 2015). We selected linear regression because of its strong fit and the residuals met the assumptions of normality and homoscedasticity. Branch length was not a significant predictor variable for foliage mass so it was dropped from the foliage model. The following models were trained with 90 branches for foliage and 89 branches for wood mass:

$$
\begin{gathered}
\ln \left(F_{\mathrm{i}}\right)=\beta_{0}+\beta_{1} \ln \left(B D_{\mathrm{i}}\right)+\epsilon_{\mathrm{i}} \\
\ln \left(W_{\mathrm{i}}\right)=\beta_{0}+\beta_{1} \ln \left(B D_{\mathrm{i}}\right)+\beta_{2} \ln \left(B L_{\mathrm{i}}\right)+\epsilon_{\mathrm{i}}
\end{gathered}
$$

where $\mathrm{F}$ is foliage wet mass $(\mathrm{g}), \mathrm{W}$ is branch wood wet mass $(\mathrm{g}), \mathrm{BD}$ is branch diameter $(\mathrm{cm})$, and $\mathrm{BL}$ is branch length $(\mathrm{m})$ for the ith branch. The $\beta \mathrm{s}$ are the estimated regression parameters, $\epsilon_{\mathrm{i}}$ is the random error, and $\ln$ is the natural logarithm. Model fit was evaluated for foliage $\left(\mathrm{R}^{2}=0.69, \mathrm{RMSE}=0.2 \mathrm{~kg}, 55 \%\right)$ and branch wood (adj $\left.\mathrm{R}^{2}=0.90, \mathrm{RMSE}=0.2 \mathrm{~kg}, 37 \%\right)$ models. We did not apply 
a correction factor for the reported negative bias caused by back-transforming logarithmic regressions (Poudel et al., 2015). Equations 1 and 2 were applied to the branch diameters and lengths of all crown branches to estimate the wet foliage and branch wood biomass in the crown. The proportion of crown foliage to crown biomass and crown branch wood to crown biomass was then multiplied by the total crown biomass and the top branches biomass. This method was used to scale crown foliage and branch wood estimates to the field measurements of total crown biomass and to estimate foliage and wood in the tree top where we did not measure branch diameter. Moisture content from the subsample branches was used to calculate dry mass for foliage and branch wood. Dry dead branch weight was calculated using the water weight of dead branch samples. We calculated the top main stem dry mass using the moisture content of the disks. Finally, the branch wood component was calculated as the sum of crown branches (live and dead), top branches (live and dead), and the top main stem. Foliage was the sum of crown and top foliage. Bole, branch wood, and foliage components were summed to get the total bone dry aboveground biomass.

\subsubsection{Terrestrial LiDAR}

Scans were collected using a FARO Focus ${ }^{3 \mathrm{D}} 120$ terrestrial LiDAR scanning unit, which is a phase-based system that collects up to 976,000 points per second with a ranging error of approximately $2 \mathrm{~mm}$. Scans were collected with a resolution of $6 \mathrm{~mm}$ at $10 \mathrm{~m}$ distance. Three to four scans were collected per tree from multiple angles selected to limit occlusion. Scan distance from the target tree was approximately $10 \mathrm{~m}$, though this distance varied dependent on tree size and stand openings. Styrofoam spheres were placed near the target tree as reference objects for scan registration.

\section{Preprocessing}

The raw point cloud data were imported into Faro SCENE (2015) to complete preprocessing before volume extraction. Within SCENE, spherical registration points were used to register the multiple scan locations. Once the registration was completed with an acceptably low placement error, the trees of interest were 

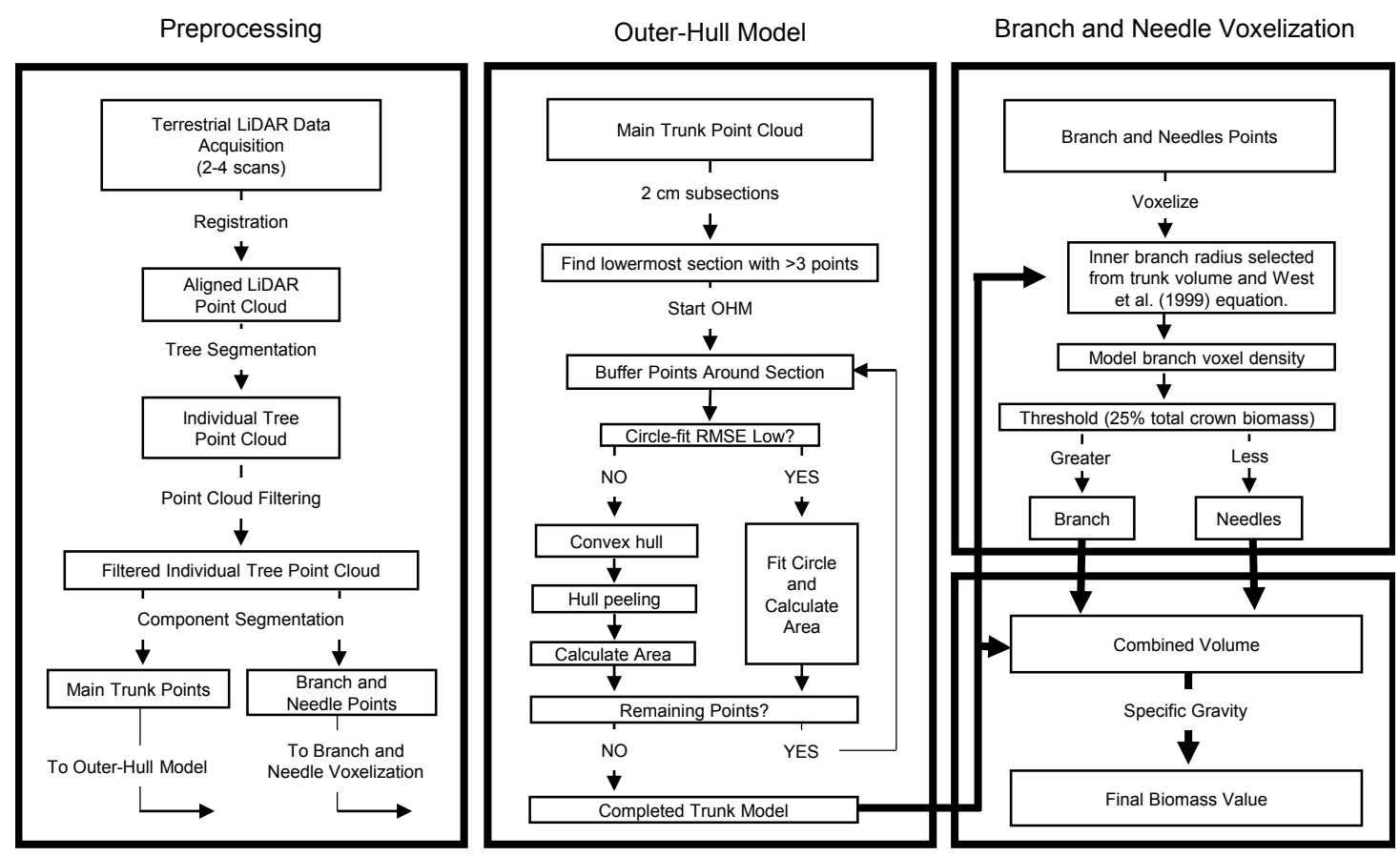

Figure 2.1: Flowchart detailing the method developed in this study to estimate tree biomass from terrestrial LiDAR scans.

segmented from the point cloud, removing the majority of noise and extraneous scan points (Figure 2.1). Stray points were filtered using two methods: [1] a custom stray point filter and [2] a statistical outlier removal filter.

Phase-based TLS units suffer from stray points in complex vegetation caused by ambiguous distance measurements in the return signal that make noiseless scan data difficult to achieve without filtering. Scans that contain fine branches or needles are particularly vulnerable to this type of noise. We filtered the tree scans using a custom stray point filter and statistical outlier removal (Appendix; Figure 2.10).

The stray point filter relies on three parameters: allocation threshold, distance threshold, and grid size (Newnham et al., 2012). The grid size defines the area to include in the filtering procedure. If a percentage of points, given by the allocation threshold fall outside the distance threshold, they are removed from the scan data. A statistical outlier removal filter was used that is based on an algorithm provided in the open source Point Cloud Library (Rusu \& Cousins, 2011). This algorithm 
functions by analyzing the neighborhood around an individual scan point for the mean distance to all surrounding points, comparing this distribution to a Gaussian distribution, with a mean and standard deviation, and removing all points whose mean distances deem them as outliers.

The use of a phase-based scanner resulted in significant noise near the central bole, so the point cloud was manually segmented the point cloud, rather than using automated methods, to differentiate between the trunk and branch points. The segment tool was used in CloudCompare to remove all branch points and expose the trunk. Often times, large portions of the central trunk were lost from the scan data due to occlusion effects. In situations such as this, the point cloud was roughly segmented leaving as many points attributed to the central bole as possible. In the case of trunk bifrication or complex branching structure on larger trees, the main large branches underwent the same segmentation process. Each tree took 10-20 minutes to preprocess. As this is a manual process, it is dependent on user skill, but the segmentation of branch from trunk points is an intuitive and relatively simple step. Finally, each component was exported in ASCII format in order to be used in the volumetric modeling process.

Tree height was estimated at this stage by subtracting the ground elevation from the 99.9th percentile of elevation in the point cloud. The use of a phasebased scanner resulted in significant noise near the central bole, so we manually segmented the point cloud, rather than using automated methods, to differentiate between the trunk and branch points (Figure 2.2). The segment tool was used in CloudCompare (2015) to remove all branch points and expose the trunk. Each tree took 10-20 minutes to preprocess. Finally, each component was exported in ASCII format in order to be used in the volumetric modeling process.

\section{Voxel-based Branch and Needle Volume Estimation}

Point-cloud voxelization is common in TLS volume quantification and is an effective means of simplifying the complex branching structure of tree point clouds into manageable volumetric units (Hosoi et al., 2013). This method directly converts points into volumetric units by dividing the point cloud into an evenly spaced grid of cubes, essentially shifting all points to the nearest cubic center. Within each 

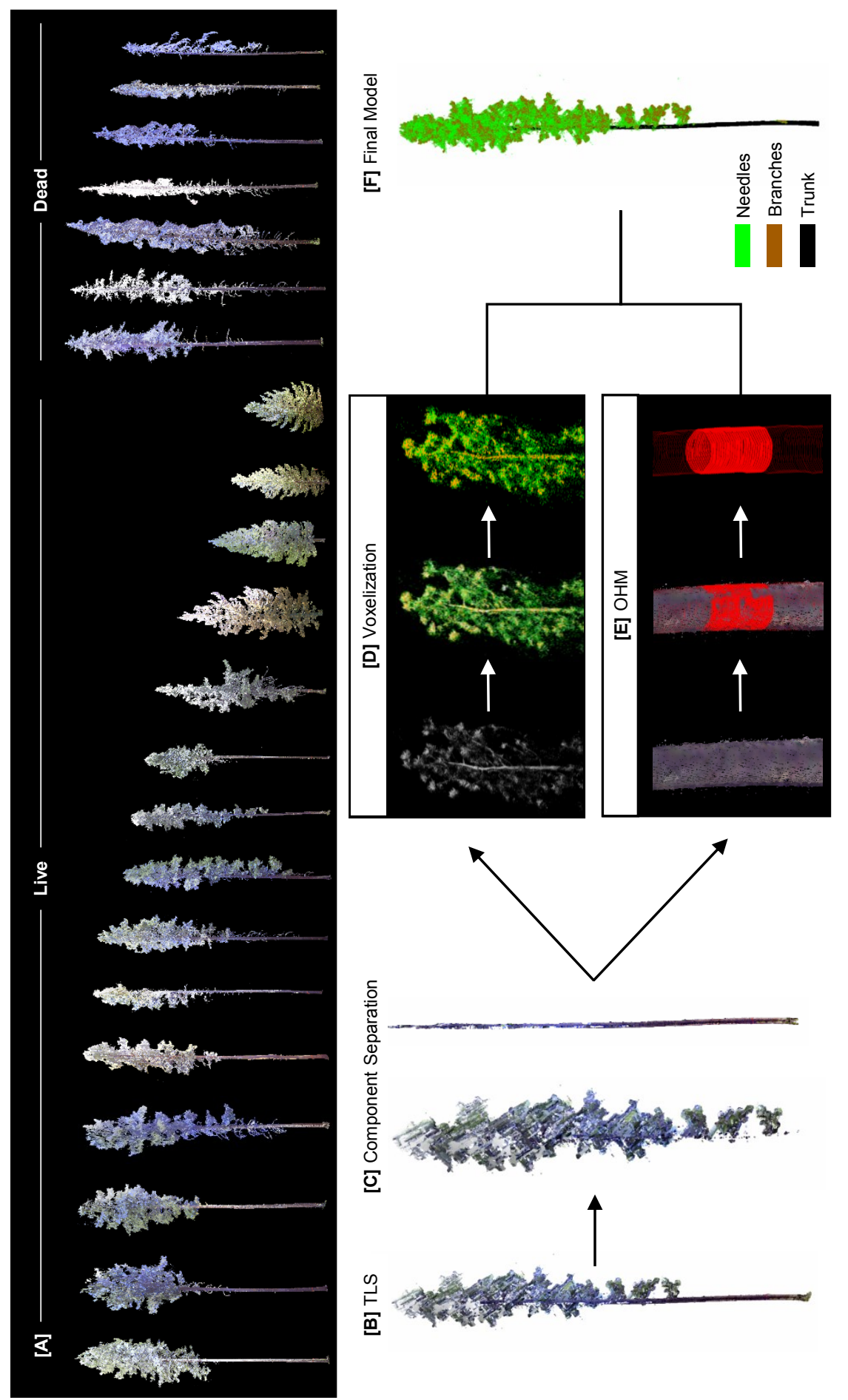

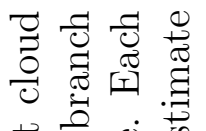

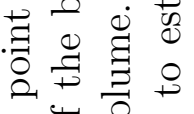
๙ 沾志声

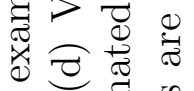

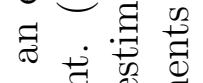
อิ चี द्वे छ्व

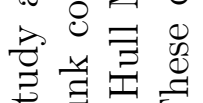
荡志式

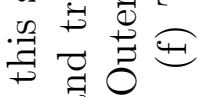
.

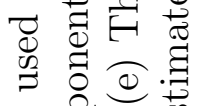
कै 宊 $\begin{array}{ccc}0 & 0 \\ 0 & 0 & 0 \\ 0 & 0 & 0 \\ 0 & 0 & 0 \\ 0 & 0 & 0 \\ 0 & 0 & 0\end{array}$ .․ㅠ
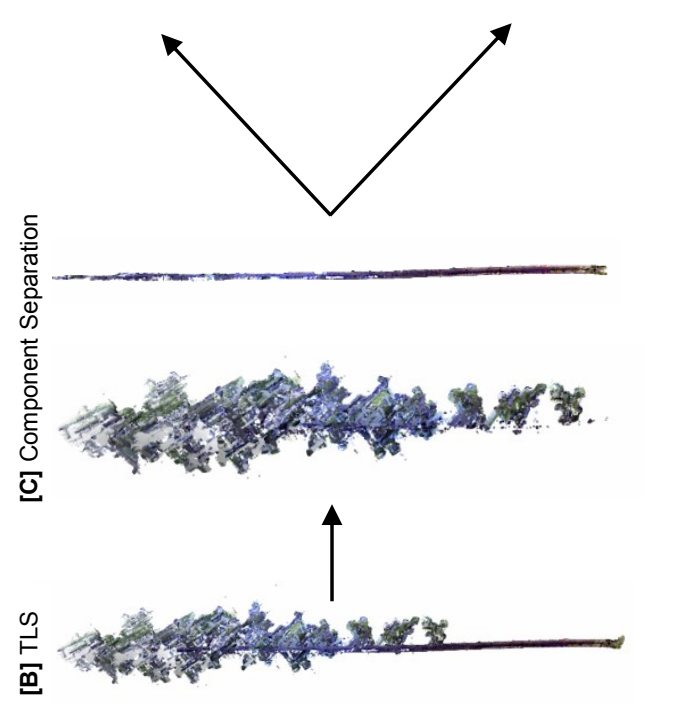
\&

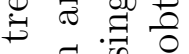
东矛

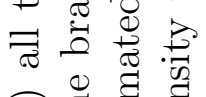

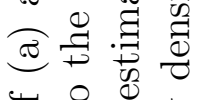

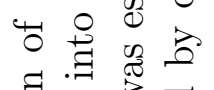

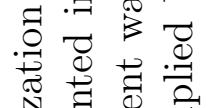
寻

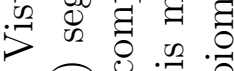

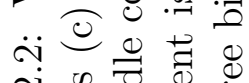

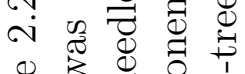

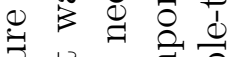
品 
voxel, we retained point density and used this information to differentiate components with high (e.g. large branches) and low point density (e.g. small branches and needles). We calculated the percent density rather than a point count within each voxel to allow direct comparison of point clouds with differing densities caused by varying scan distance and resolution. The percent density was calculated as the total number of returns within an individual voxel divided by the maximum possible number of returns within any voxel. We filtered the point cloud to create an even grid spacing of $5 \mathrm{~mm}$ and subsequently selected a $2 \mathrm{~cm}$ voxel size for all trees. Selecting this voxel size balanced the tradeoff between captured voxel density variation and spatial resolution. Fine voxel resolution enhances spatial detail, but reduces the range of voxel densities, while larger voxels have a coarse resolution that increases the range of densities found in a voxelized point cloud.This combination of filtering and voxel size allowed for a potential maximum of 64 returns per voxel, which captured fine variation in point density while retaining high enough spatial resolution to differentiate between large branches and small branches and needles. The voxels with the highest density were considered to be large branches and voxels below this threshold were treated as small branches or needles. The algorithm automatically selects a threshold for each tree such that approximately $25 \%$ of the total branch and needle volume was attributed to needles, based on the Jenkins et al. (2003) component ratio estimates.

The distribution of voxel density from crown center to edge will approximate the branch volume distribution if fully and evenly covered by TLS points since point coverage is a function of branch surface area (Figure 2.3). However, clumping of foliage and direct occlusion from branches significantly reduces return density in the inner crown. If left uncorrected, the exceedingly low percent density of the inner crown underestimates branch volume. Voxel percent density is corrected with a three step process: [1] use height specific trunk radius to estimate inner branch size using a relationship derived from West et al. (1999), [2] use inner branch size with a logarithmic curve to approximate the distribution of branch size and local variability along the horizontal length of the tree crown, and [3] correct the horizontal mean percent density distribution with the logarithmic model.

Inner crown occlusion is mitigated by estimating inner branch radius using West, Brown, Enquist (WBE) allometric scaling theory (West et al., 1999). West 


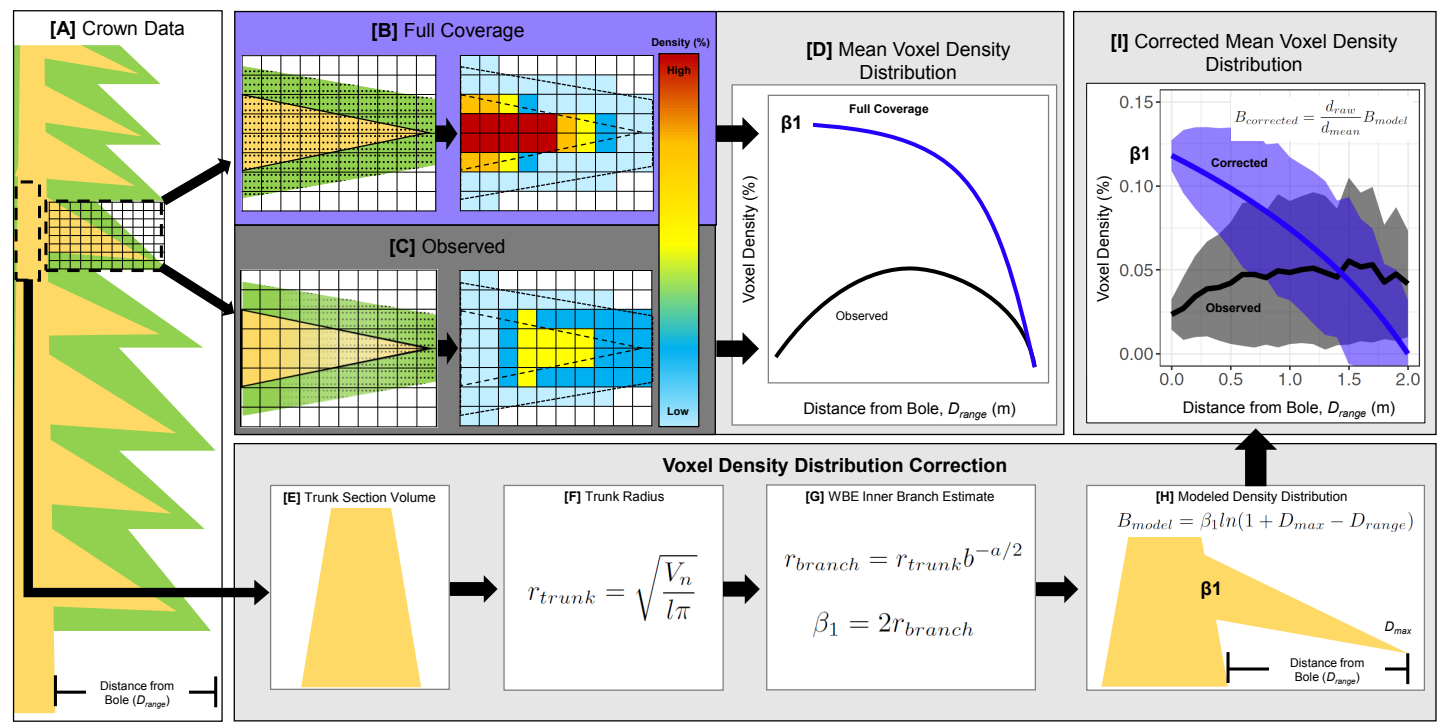

Figure 2.3: Flowchart detailing the method of crown branch and foliage biomass estimation. The $[\mathrm{A}]$ crown returns under a $[\mathrm{B}]$ full coverage scenario are expected to be proportional to total volume, but $[\mathrm{C}]$ occlusion reduced the point coverage in the inner crown. The $[\mathrm{D}]$ voxel density is not representative of branch volume in the presence of significant occlusion effects. [E] The observed mean horizontal voxel density distribution is corrected using the height-specific trunk volume. $[\mathrm{F}]$ Trunk radius is used with $[\mathrm{G}]$ WBE allometric scaling theory to estimate the approximate radius of an inner crown branch $\left(r_{\text {branch }}\right)$ and then innermost mean voxel density $\left(\beta_{1}\right)$. Voxel density represents the percent of filled space in a $2 \mathrm{~cm}$ cube and can be directly converted to an estimate of branch volume. The average innermost crown voxel density is used in a $[\mathrm{H}]$ logarithmic model spanning the horizontal distance from the bole to the crown edge. [I] The corrected distribution is modeled from the observed and modeled distributions, correcting crown voxel density for occlusion effects.

et al. (1999) theorized that the radius of a specific branching order can be approximated for a given trunk radius if the tree grows as a hierarchical structure. We used the radius of the trunk to estimate the largest or 2nd order branch size in each $1 \mathrm{~m}$ crown subsection with the equations from West et al. (1999). The estimate of the inner branch voxel density was made with:

$$
r_{\text {trunk }}=\sqrt{\frac{V_{n}}{l \pi}}
$$




$$
\begin{gathered}
r_{\text {branch }}=r_{\text {trunk }} b^{-a / 2} \\
\beta_{1}=2 r_{\text {branch }}
\end{gathered}
$$

Where $r_{\text {trunk }}$ is the mean trunk radius at each $1 \mathrm{~m}$ vertical subsection, $V_{n}$ is the trunk volume at each subsection, and $l$ is the length of the subsections. $r_{\text {branch }}$ is the mean inner branch radius at each $1 \mathrm{~m}$ vertical subsection, $b$ is the branching order of which size is estimated, and $a$ is a parameter relating daughter to parent branches ( $a=2$, is area preserving). For this study, we estimated the inner branch radius of 2 nd order branches that are area preserving $(a=2$ and $b=2$ in Equation 2.4). $\beta_{1}$ is a coefficient estimating the innermost mean voxel density as the branch diameter closest to the trunk.

The largest branch size $\left(\beta_{1}\right)$ and horizontal crown extent are used as parameters for the logarithmic model estimating the horizontal mean density distribution (Figure 2.3). The normalized density percent values are then multiplied by the logarithmic model for a corrected estimate of the mean density distribution with local variability in voxel density captured. We use $\beta_{1}$ to create a logarithmic correction model of the mean voxel density distribution in the form:

$$
B_{\text {model }}=\beta_{1} \ln \left(1+D_{\text {max }}-D_{\text {range }}\right)
$$

Where $\beta_{1}$ is the innermost mean voxel density from Equation 2.6, $D_{\max }$ is the maximum distance of crown branches from the central bole for crown subsections, and $D_{\text {range }}$ is the horizontal $10 \mathrm{~cm}$ intervals over which voxel density is calculated. $B_{\text {model }}$ replaces the mean percent density distribution and the residuals of normalized density percent guide the local variability in branch size.

We normalize the percent density by the mean percent density and subsequently correct the distribution with our modeled mean voxel density distribution with:

$$
B_{\text {corrected }}=\frac{d_{\text {raw }}}{d_{\text {mean }}} B_{\text {model }}
$$

Where, $d_{\text {raw }}$ is the voxel density as calculated on the filtered crown point cloud 
and $d_{\text {mean }}$ is the mean horizontal voxel density distribution as derived from each individual $1 \mathrm{~m}$ subsection of the crown. Voxels are finally converted to volume by multiplying corrected percent density by voxel volume. Our approach of informing the branch modeling with the output of the OHM algorithm provides realistic crown volume estimates based primarily on the trunk volume and theoretical tree scaling relationships.

\section{Outer Hull Model for Trunk Volume Estimation}

The Outer Hull Model (OHM) algorithm presented in this manuscript functions on very simple principles, relying primarily on an iterative convex hull procedure (Figure 2.2). Essentially, the model takes the following approach: [1] initialize by locating the lowermost set of three or more points within a specified thickness, [2] fit a circle if the quality of fit is below a user-specified root mean squared error (RMSE) threshold, [3] if the circle-fitting fails, apply a convex hull algorithm to the segmented points, [4] use convex hull peeling to remove noise points, and [5] calculate the area of the peeled convex hull and multiply by the slice thickness to retrieve volume (Figure 2.4). The following sections describe these individual steps.

The trunk points are sectioned along the z-axis in even intervals in order to facilitate the iterative modeling process. The result of this first step creates evenly spaced horizontal slices of TLS returns that can be modeled. The algorithm begins at the first newly created section containing 3 or more points, starting from the lowest section and increasing along the z-axis.

Each section of the trunk point cloud is evaluated to determine if the best fit is obtained by a circle or the convex hull. We determine this by using a least squares circle fit algorithm (Coope, 1993). Under circumstances of low noise and highly cylindrical tree shapes this form of modeling provides an excellent method of determining tree diameter and volume from TLS data (Tansey et al., 2009). The model calculates a convex hull rather than a circle if one of two conditions is met: (1) the circle fitting exceeds an RMSE threshold or (2) the circle has a larger diameter than field-measured DBH. These cases of the model using a convex hull rather than a circle address instances where trunks are not circular or when the 


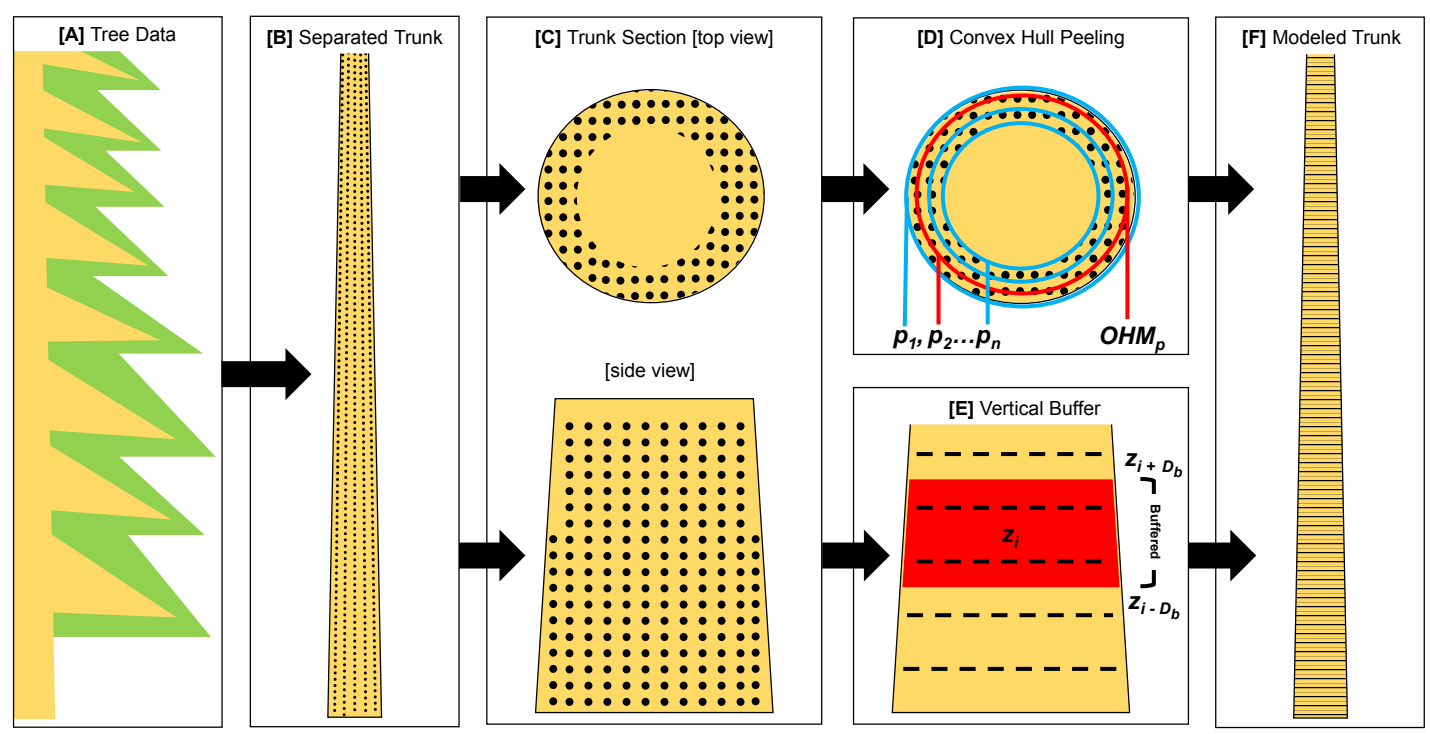

Figure 2.4: Flowchart detailing the method of trunk biomass estimation. [A] The TLS data is separated to obtain the [B] trunk point cloud and the modeling process is initiated. The algorithm begins from the lowest point cloud height and, moving upward, finds the first $[\mathrm{C}]$ trunk subsection height $\left(z_{i}\right)$ with three or more points within a specified thickness. [C] A circle is fit to the subsection points and, if the error in fit exceeds a threshold, the convex hull is computed and points are removed that fall along this initial outer hull. [D] The point removal process continues iteratively $\left(p_{1}, p_{2} \ldots p_{n}\right)$, "peeling" the outermost points of the set until the percent of returns removed reaches a threshold $\left(O H M_{p}\right)$ defined by the calibration process. Occluded portions of the trunk sections $\left(z_{i}\right)$ are partially filled using a [E] vertical buffer $\left(D_{b}\right)$ that includes trunk points from above $\left(z_{i+D_{b}}\right)$ and below $\left(z_{i-D_{b}}\right)$ for use in the convex hull peeling procedure. The algorithm proceeds until all trunk subsections are modeled $[\mathrm{F}]$.

fitted circle diameter is artificially large due to noise. The convex hull procedure is exclusively used below DBH height since most trees become less cylindrical, exhibiting some degree of flaring, as the stem transitions to the rooting structure.

We used a two-dimensional convex hull algorithm (Eddy, 1977) for each subset of points delineated during the slicing procedure. A convex hull is the outermost area that encloses a finite set of points that may exist in two-dimensional to ndimensional space. The polygon that is created from the algorithm defines the outermost points of the trunk slice of which area can easily be calculated. The convex-hull method contrasts with cylinder or frustum fitting algorithms in that 
the shape of the point set at each height interval defines the shape of the modeled surface, rather than relying on a best-fit primitive. This method is preferable to cylinder fitting when point coverage of the trunk is sufficient because irregular trunk shapes are quantified with greater certainty.

We improved the convex hull estimation method with two approaches: [1] slice buffering and [2] convex hull peeling. The variable distance buffer acts as a smoothing filter, encompassing all points within a set vertical distance around the trunk slice being modeled. The buffering approach reduces occlusion effects and model robustness by increasing the number of points being modeled. We used a single $20 \mathrm{~cm}$ buffering distance for every tree since this value filled most gaps from occlusion, but retained the unique trunk shape.

Terrestrial LiDAR datasets that have incomplete coverage of returns from the trunk section due to occlusion effects require an alternative method to help reduce gaps in data. Since this algorithm uses a convex hull method, the point set will always be enclosed, regardless of the shape of the sliced trunk in the point cloud. This function is of great benefit because area, and thus volume, can be computed at any trunk section, assuming it contains 3 or more points. However, in situations where point coverage is incomplete and large sections of the trunk are unmeasured there can be significant volume underestimation, because the polygon created from the convex hull process will simply connect the next closest and outermost point, which may not be representative of the reality of the trunk shape. A convex hull method of gap-filling has been used in a modeling algorithm applied to mangrove trees (Olagoke et al., 2016), however the significant gaps present from occluded trunk points in our data would have produced unacceptable volume errors.

In order to reduce this source of error we implement a buffering procedure at each successive slice that the convex hull was computed for. The buffer functions by including all points above and below the slice being modeled, increasing the likelihood of the outer trunk points being included in the final model. The distance of the buffer can be varied according to the coverage of LiDAR returns and level of occlusion present on the individual tree. Increasing the buffer produces a smoothing effect on the final model that is ideal in circumstances of occlusion, but may alter the accuracy of the representation of trunk shape. This buffering process has the added benefit of increasing the likelihood that the modeling procedure 
will complete at each section because sections with less than 3 points will likely increase in point density. The option for buffering at each successive trunk slice is essential for providing the most complete and representative volumetric model of the central bole of the tree.

We implement what is, to our knowledge, the first example of convex hull peeling in the context of volume quantification of trees using terrestrial LiDAR, in hopes of reducing the influence of noise in the data and subsequent bias. Convex hull peeling is used in computational geometry to give order to non-organized point sets by calculating the convex hull of the point set and then recalculating the convex hull without the points that fall on the first convex hull (Chazelle, 1985). With each iteration of the process, layers within the data are created, allowing for the underlying structure to be analyzed. For our purposes, this method is useful for simultaneous noise removal and volume quantification. The convex hull peeling process removes these outermost points attributed to noise and continues this process iteratively until the innermost points remain.

A threshold is set to determine the number of convex hull peeling iterations based on the desired percentage of points to remove from the original cloud. If this threshold is set to $0 \%$, the first and outermost convex hull is used - essentially turning off the peeling process. A peeling threshold of $100 \%$ will eliminate all points and no convex hull will be calculated. A conservatively low threshold of $5 \%$ is effective in low noise point clouds and will reduce spurious noise points, but, with noisier data, higher thresholds such as $45 \%$ are appropriate. If the threshold is set too high and too many peeling iterations occur there is the potential for the removal of a significant number of true trunk points, so a moderately low threshold is preferable to an extremely high threshold.

The calibration for the OHM algorithm required a single tree to determine the best balance between the peeling and buffering process. The convex hull peeling process removes spurious points and the buffering process increases the number of points. It follows that the two procedures will interact. Substantial occlusion within the trunk point cloud requires a larger buffering region, but also a greater number of peeling iterations in order to reduce the likelihood of volume overestimation. The calibration procedure used the known trunk biomass in order to find this balance under a range of occlusion scenarios. We modeled the interaction 
between buffering and convex hull peeling with a power function in the form:

$$
O H M_{P}=a D_{b}^{b}
$$

Where $O H M_{P}$ is the convex hull peeling threshold, $D_{b}$ is buffer distance, and $a$ and $b$ are the model coefficients. This approach requires the user to provide only the buffering distance needed to reduce occlusion effects.

The final steps of the modeling process are the same regardless of the procedure implemented on each individual vertical section. Once the area of the section has been determined by either convex hull or circle fitting, the height of the section is used to estimate volume. Each successive section is modeled until the volume of the entire trunk is determined. We use this same procedure to estimate diameter though the length of the trunk; DBH is estimated at $1.3 \mathrm{~m}$ from the lowest point in the LiDAR data. The area calculation of each section is converted into a filled point cloud representation of the modeled tree that can be visualized, allowing for comparison to the raw point cloud to identify errors that would produce significant deviations from actual biomass.

\section{Validation of Non-destructive Biomass Estimates}

We converted volume to biomass for all tree components using an average lodgepole pine specific gravity of 0.38 (Chojnacky et al., 2014). We evaluated error using this average specific gravity by comparing to biomass estimates generated from measured per-tree density values. Though density was calculated on the bole sections alone, average per-tree density values were applied across all components. Biomass estimates derived from the TLS data were compared to destructivelysampled biomass estimates to evaluate model performance. For consistency, we used a single set of input parameters for every tree modeled, rather than relying on parameter selection by the user. Bias, RMSE, and RMSE\% were reported using:

$$
\text { bias }=\frac{\sum_{i=1}^{n}\left(y_{i}-\widehat{y}_{i}\right)}{n}
$$




$$
\begin{gathered}
R M S E=\sqrt{\frac{\sum_{i=1}^{n}\left(y_{i}-\widehat{y}_{i}\right)^{2}}{n}} \\
R M S E \%=\frac{R M S E}{\widehat{y}_{i}}
\end{gathered}
$$

Bias gave an indication of the tendency of our approach to over- or underestimate biomass. RMSE was reported both in $\mathrm{kg}$ and as a percentage of the mean of the sample to provide a clear comparison to other methods of non-destructive biomass estimation using terrestrial LiDAR. The RMSE difference in biomass estimates between the two density values were determined for the whole tree and each component. The density effects are reported as total difference in RMSE, both in $\mathrm{kg}$ and as a percentage.

\subsection{Results}

\subsubsection{Diameter and Height}

The convex-hull peeling approach produced an accurate (Figure 2.5a) and unbiased (bias $=0.0 \mathrm{~cm}$ ) estimate of trunk diameter when compared to the field measurements (Table 2.2). The circle-fitting RMSE threshold of $10 \mathrm{~cm}$ was exceeded $63 \%$ of the time, requiring the convex-hull peeling approach. Tree height estimates were also very accurate (Figure 2.5b; Table 2.2). Height estimates were negatively biased by $30 \mathrm{~cm}$ due to a single outlier in the live tree subsample (Figure 2.5b). Excluding this outlier reduced bias to $-16 \mathrm{~cm}$ and RMSE to $0.3 \mathrm{~m}$ for all trees. Across all samples, diameter and height error was low, but living trees had higher error than dead trees. The dead samples were unbiased.

\subsubsection{Parameter Optimization}

A single tree was used to parameterize the OHM algorithm. The number of peeling iterations were negatively correlated with biomass estimates, while buffering distance was positively correlated (Figure 2.6). In order to achieve low error trunk 

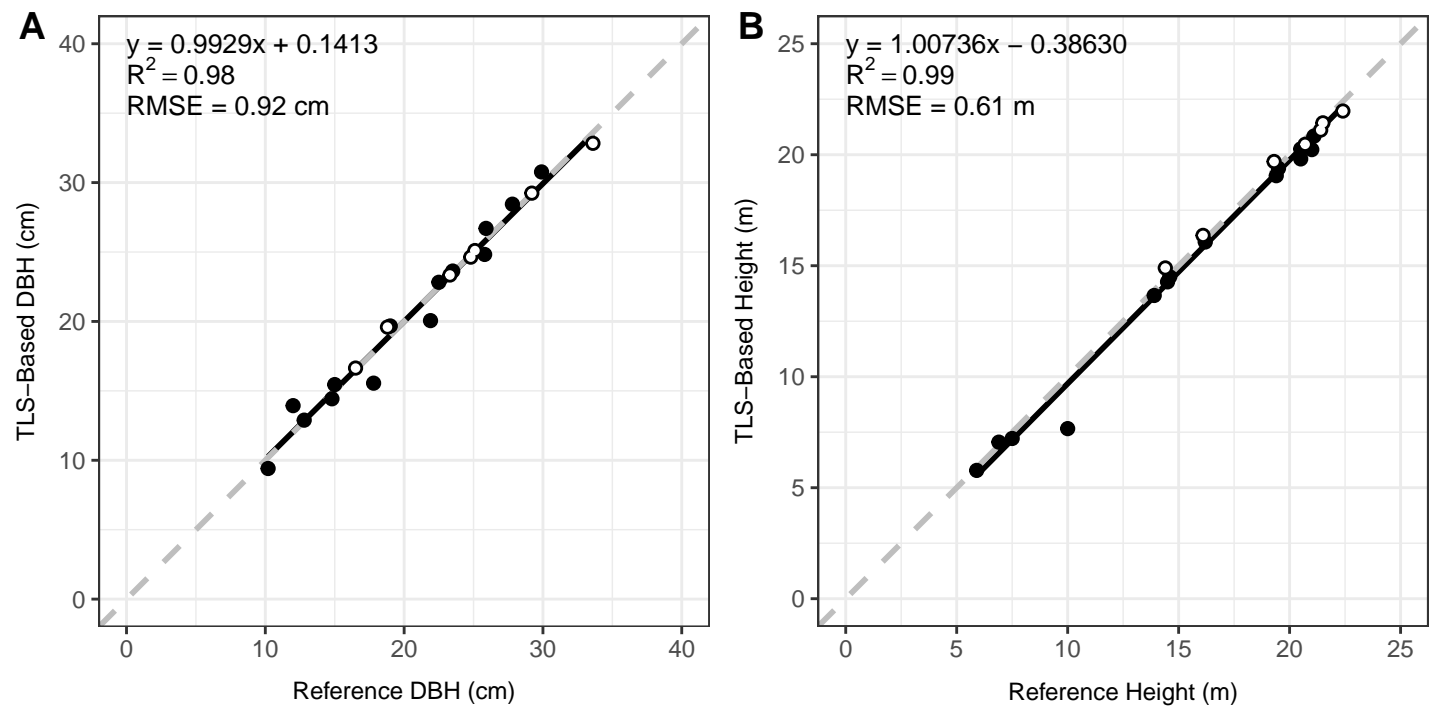

Figure 2.5: Comparison of field measured and TLS-based estimates of (a) diameter at breast height and (b) height for living (black) and dead (hollow circles) trees.

biomass estimates, the percentage of points removed through peeling must increase as buffering distance increases; in essence, buffering increases the number of points that must be removed through the peeling operation. The peeling percentage stabilized near $60 \%$ above $50 \mathrm{~cm}$ of buffering. The relationship between buffering distance and peeling iterations was best described using a power function $\left(\mathrm{R}^{2}=0.99\right)$. The computation time of this process was roughly exponential, increasing with buffering distance. The $20 \mathrm{~cm}$ buffer used for the trunk modeling procedure required five seconds to 53 seconds for processing, averaging 25 seconds per tree and increasing linearly with the number of trunk points in the point cloud.

\subsubsection{Validation of Non-destructive Biomass Estimates}

Whole-tree aboveground biomass estimates derived from the terrestrial LiDAR scans strongly agreed with the destructive sampling estimates (Figure 2.7; Table 2.2). The OHM method performed well when estimating tree trunk biomass (8.4\%), but RMSEs (\%) were higher for branch (49.4\%) and needle (38.9\%) biomass (Figure 2.8). When branches and needles were combined as a single component, the model performed slightly better (Table 2.2). LiDAR estimates of biomass 

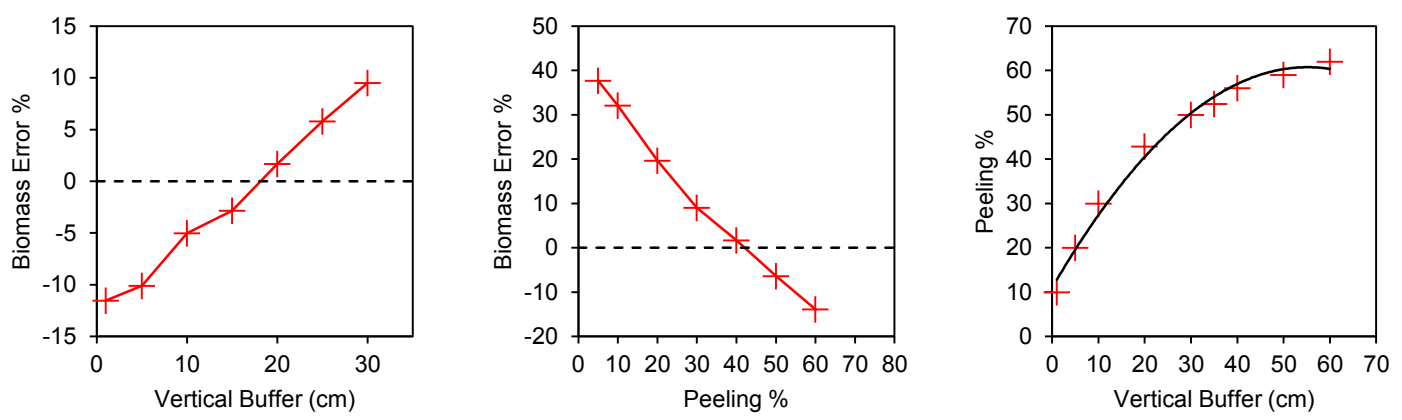

Figure 2.6: Parameter optimization process used in the convex hull peeling algorithm. Biomass error was compared to the primary input parameters for each volume estimation approach. Red lines approximate the effect of varying input parameters on biomass error for the single tree used for calibration. The final column (right) shows the final optimized model based on the training subsample used to automate parameter selection.

were more accurate for dead trees than live trees for whole tree $(3.3 \%)$, woody $(3.2 \%)$, and trunk (7.5\%) biomass (Table 2.2$)$. All biomass estimates were nearly unbiased except branch and branch with needle biomass, which consistently underestimated. All dead trees had lost their needles before sampling, so the needle component could not be compared between live and dead trees. The average pertree density reduced the trunk RMSE to $7.3 \%$, with dead trees averaging $3.0 \%$. The application of per-tree density tended to only slightly improve RMSE for other components.

\subsection{Discussion}

The approach presented in this study combined voxelization and the OHM to accurately and non-destructively estimate the aboveground biomass of needleleaf trees using terrestrial LiDAR with minimal user input. We derived nearly unbiased and highly accurate diameter and height measurements from the LiDAR data using an automated method. Biomass estimation required manual segmentation of the central bole, but this preprocessing time was minor relative to the time and cost associated with destructive sampling. Once the central bole was segmented, the convex hull peeling approach was used to accurately recreate its unique exterior 


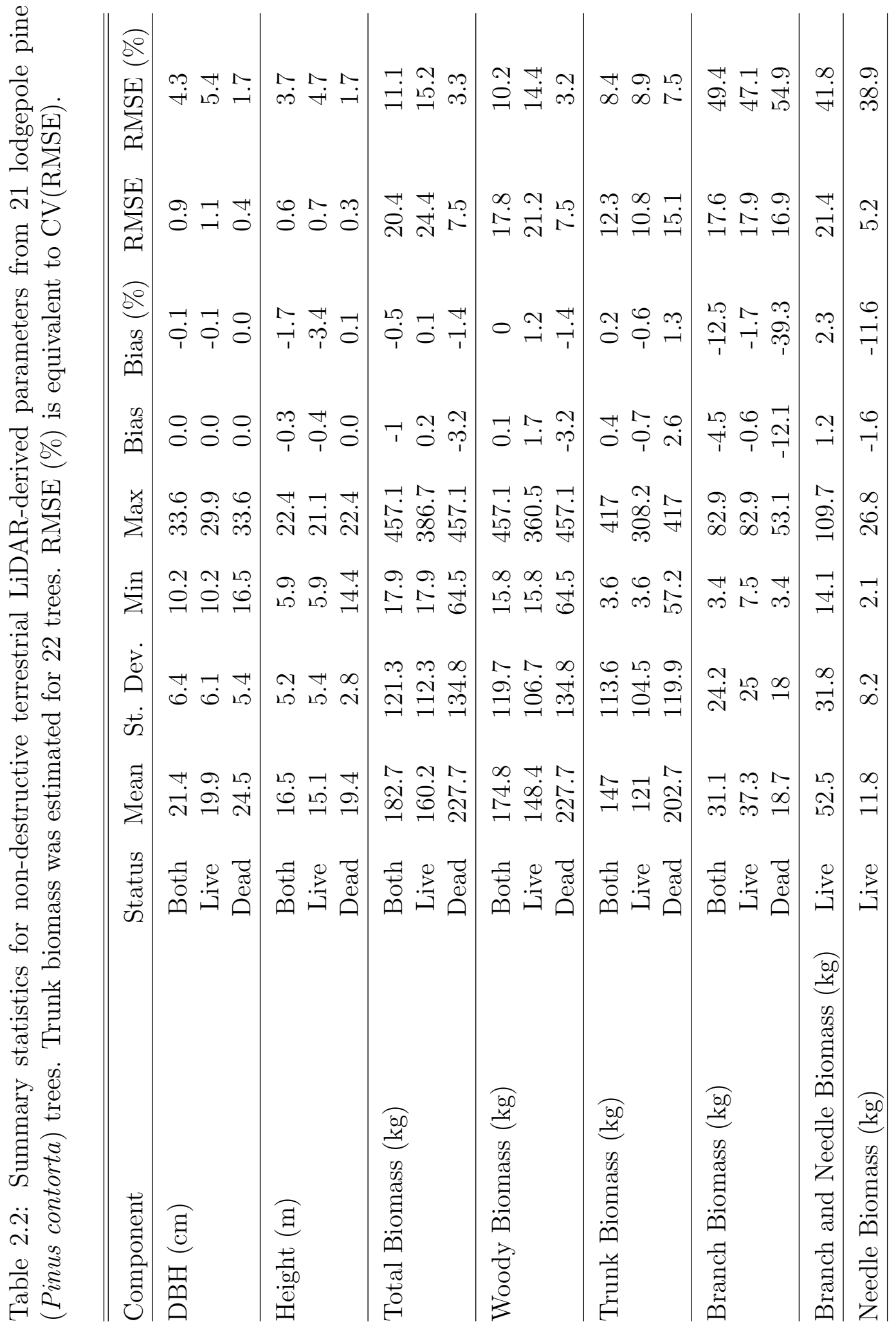



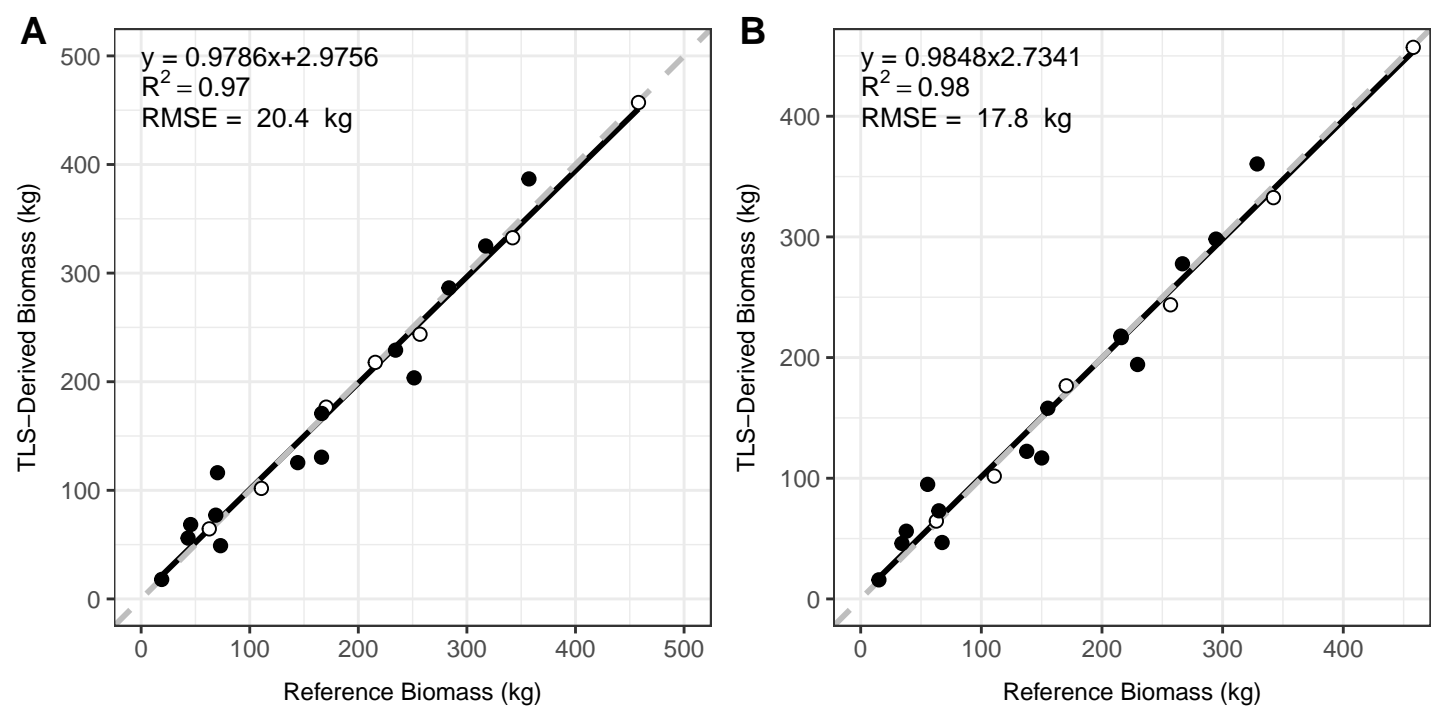

Figure 2.7: Non-destructive (a) whole-tree and (b) woody aboveground biomass estimates with terrestrial LiDAR compared to destructively sampled reference biomass for living (black) and dead (open-circles) trees.

structure - ideal in circumstances of non-cylindrical tree structure. Needle and branch biomass estimates were derived using a voxelization subroutine. Branch biomass estimates had higher RMSE, but were relatively unbiased considering significant inner crown occlusion and noise present in the raw point cloud. Error of every component was lower overall for dead trees. Density was not a major control over biomass variability, but inclusion of individual tree density measurements did reduce error.

\subsubsection{Basic Forestry Measurements}

Diameter at breast height was accurately estimated using the same convex hull peeling algorithm that forms the basis for the OHM. The major sources of diameter measurement error using terrestrial LiDAR, and phase-based scanners in particular, were exhibited in this dataset: low return density, high noise, and high occlusion. At breast height, point cloud density was high and occlusion was low in larger diameter trees, allowing for accurate diameter measurement. Alternatively, the smallest trees had the greatest error since foliage was dense at breast height 

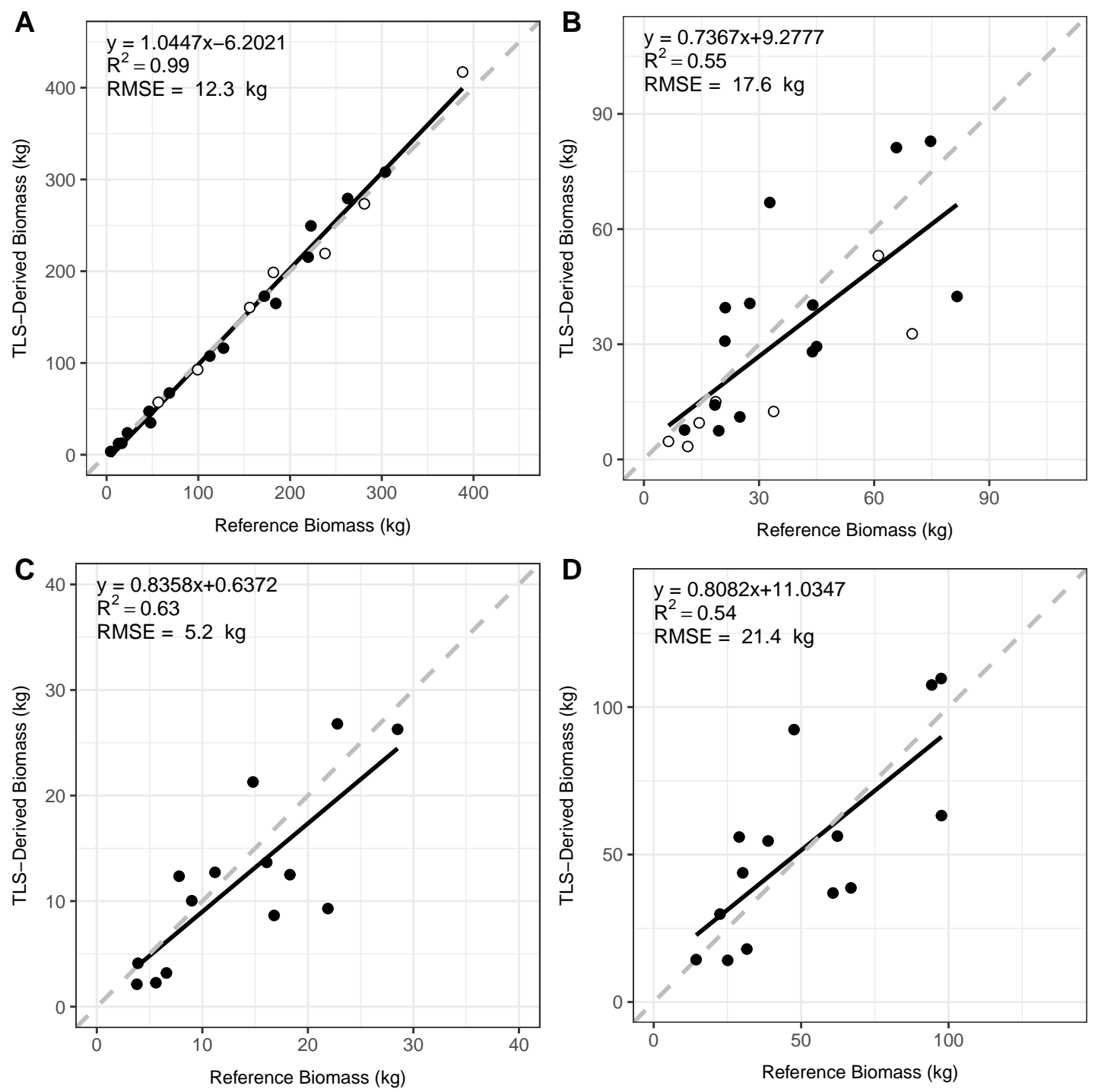

Figure 2.8: Comparison of destructively-sampled and non-destructive TLS-based estimates of (a) trunk, (b) branches, (c) needles, and (d) branches with needles for living (black) and dead (open-circles) trees. 
(Figure 2.5), reducing point density and increasing occlusion at the trunk. Despite these issues, the error in diameter estimation is exceptionally low and unbiased.

The percentile approach successfully retrieved height from the TLS point cloud, with the exception of one outlier. This underestimation had no returns present at the correct height, suggesting too many points were filtered during preprocessing. Our method outperformed common manual (Hopkinson et al., 2004) and automatic (Calders et al., 2015) approaches of estimating height with TLS and were also more accurate than tree height estimates collected with airborne LiDAR in conifer stands (Falkowski et al., 2006), albeit airborne LiDAR spans a much broader area. The strong performance of our height estimation may be due to the relatively low height of the stand sampled, selecting trees with a clear line-of-sight, and scanning the trees from multiple directions. We anticipate multiple return instruments would have even lower error. The use of TLS for height measurement should be preferable to manual methods of validating tree height at the plot level using airborne or spaceborne LiDAR.

\subsubsection{Non-destructive Biomass Estimation}

Several recent publications have successfully non-destructively estimated biomass with cylinder-fitting (Calders et al., 2015; Hackenberg et al., 2015a; Yu et al., 2013), but the same algorithm applied to our dataset produced undesirable results as representations of tree structure were not realistic or reliable (Hackenberg et al., 2015b). We developed an alternative approach that uses minimal user input to accurately estimate biomass of individual tree components. Trunk form was retained with the OHM algorithm as it relies on the exterior returns to create a solid volumetric model. This unique form is lost with cylinder-fitting approaches, potentially becoming a major source of error in some species or individuals with non-cylindrical trunks. Our OHM approach is effective and accurate under conditions of noisy scan data, where cylinder fitting is often unpredictable. Trunk estimates were even reliable in the canopy, where trunk occlusion is high (Figure 2.9). We cannot yet evaluate the accuracy of the OHM algorithm with respect to broadleaf species, but anticipate success under leaf-off conditions. The primary drawback of our algorithm in the current form is the inability to differentiate be- 
Table 2.3: Comparison of the RMSE (\%) of the Outer Hull Model algorithm to other algorithms validated with destructive samples. Only trunk biomass RMSE $\%$ was compared to minimize bias from higher uncertainty foliage estimates.

\begin{tabular}{lcc}
\hline \hline Publication & RMSE (\%) & Tree Type \\
\hline Calders et al. 2015 & 16.1 & Broadleaf \\
Hackenberg et al. 2015a & 15.3 & Broadleaf \\
Yu et al. 2013 & 12.5 & Needleleaf \\
OHM Algorithm & $\mathbf{1 0 . 2}$ & Needleleaf \\
\hline
\end{tabular}

tween branches and trunk, requiring manual segmentation for accurate biomass estimates. This could become a cumbersome task for trees with a high branching order. However, this is a minor issue for trees that have a central bole where manual segmentation is a simple and efficient process.

The OHM algorithm is one of the most accurate methods of estimating stem volume and biomass. Our method performs better than other studies that validate biomass estimates from LiDAR with destructive sampling data (Table 2.3). The low RMSE of our algorithm can partially be attributed to the greatest contribution of biomass in our samples existing in the trunk, reducing the complexity and potential for error when modeling this component. Broadleaf and higher order branching trees tend be more difficult to model accurately, ultimately increasing model uncertainty (Calders et al., 2015; Hackenberg et al., 2015a). Further reductions in error were obtained when the OHM algorithm was used in concert with measured wood density of the trunk component; RMSE for the trunk decreased to $7.3 \%$. Error was further reduced to $3.0 \%$ for dead trees . To our knowledge, this is the first attempt to validate branch and needle biomass estimates from TLS with coincident destructive sampling on the whole-tree scale. Other work has related point cloud return density to destructive measurements of juvenile trees, but did not attempt a direct estimate of individual components (Seidel et al., 2011). A voxelization approach has been used to estimate wood volume and leaf area density, but these measurements were not extensively validated with destructive sampling and thus cannot be directly compared to our approach (Hosoi et al., 2013; Omasa et al., 2006). While the raw point cloud data was greatly influenced 


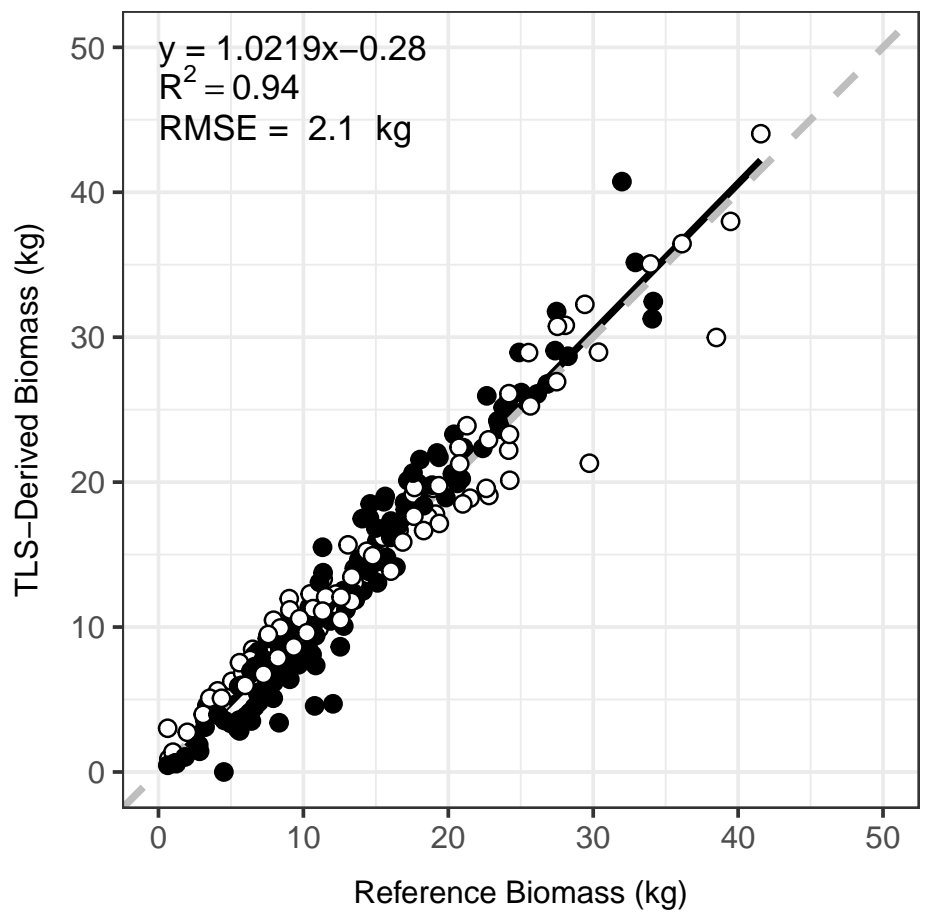

Figure 2.9: Comparison of destructively sampled and non-destructive TLS-based estimates of trunk section biomass $(n=232)$. Black circles are live tree samples and open circle are dead tree samples.

by inner crown occlusion, our method of modeling the branch size distribution using information from the trunk allowed crown biomass to be estimated without the need for empirically derived calibration. This method of branch biomass estimation in needleleaf trees is a clear improvement over QSM methods in high noise scenarios where biomass can be extremely overestimated. Branches and needles contained an average of approximately $20 \%$ of the whole tree biomass, but this contribution decreased with increasing tree size, which may reduce overall biomass uncertainty when modeling larger trees since our trunk estimates were accurate.

The OHM can potentially be applied to most trunk structures without further calibration for accurate biomass estimation. Parameter selection and calibration was based on a single destructively-harvested tree. Since the OHM is modeling a variably occluded stem structure, the OHM parameters may not change significantly for other tree species and growth forms. Our final calibrated algorithm required the buffering extent as the only user defined parameter when model- 
ing biomass. The component voxelization procedure only required height specific trunk volume to accurately estimate biomass, but needs further validation with other needleleaf species. Species specific variation in the relationship between trunk volume and inner branch radius needs to be captured for this method to be applied universally across a range of needleleaf tree structures. Future work will focus on fine tuning the voxelization approach to other species for more precise biomass estimation.

We included seven dead trees as an attempt to emulate leaf-off conditions for a needleleaf species and reduce uncertainty in woody biomass estimates. As expected, whole tree and woody biomass estimates had lower error in dead trees. Root mean squared error decreased by nearly $8 \%$ when dead samples were used. The occlusion effects at the trunk were lower in the dead trees as needles were not present to block the majority of returns. Our findings suggest that dead trees may be useful for non-destructively studying the structural allocation of biomass with terrestrial LiDAR.

Wood density is widely known to cause significant variation in the accuracy of biomass estimation especially across continental scales (Mitchard et al., 2014). Wood density contributed to approximately $1.3 \%$ of the trunk biomass estimation uncertainty. While this increase in accuracy is significant, coincident fieldmeasured density subsamples may not provide these same improvements in estimation error; within tree density variability can contribute as much or more to biomass uncertainty. Several subsample-based density estimation approaches using tree cores have been proposed, but are only useful with a priori knowledge of the tree species characteristics (Williamson \& Wiemann, 2010). Moreover, although trunk RMSE improved when applying the tree specific density, whole tree biomass uncertainty increased slightly, suggesting density differences among components of the tree. As such, we suggest that species-specific wood density estimates are adequate when coincident field measurements are not feasible.

This approach to non-destructive estimation of aboveground biomass of trees can potentially reduce uncertainty within current allometric equations by allowing for more efficient sampling of single-tree biomass. The logistical and financial limitations surrounding destructive sampling are reduced substantially with the method introduced in this work. We approximate that biomass estimation us- 
ing the method in this study is 16 times faster than destructive sampling. These time savings increase substantially with larger trees. Moreover, one of the primary issues surrounding allometric equation development is a high coefficient of variation caused by insufficient sample size (Chave et al., 2004); non-destructive sampling with terrestrial LiDAR can significantly increase sample size, in turn reducing uncertainty. Currently, national-scale allometric equations are widely applied, but local applications of these relationships may result in significant errors (TerMikaelian \& Korzukhin, 1997; Jenkins et al., 2003; Chave et al., 2005; Chojnacky et al., 2014), which are exacerbated when applied to trees outside of the measured diameter, height, and geographic range. The development of high sample size non-destructive allometric equations that include trees that span the realistic range of diameters found in forests have far-reaching implications. Application of these relationships to identified high-uncertainty regions may be one of the most efficient methods of improving confidence in global estimates of aboveground biomass.

\subsection{Conclusion}

Our approach to non-destructively estimate biomass using terrestrial LiDAR with voxelization and the OHM algorithm was nearly unbiased and had exceptionally low error. Density controlled variability across the samples in this study and reduced estimation error, but applying an average species-specific density still produced low error biomass estimates. Accurate and efficient non-destructive biomass estimation has the potential to reduce uncertainty in local allometric equations by greatly increasing sample size over large areas with minimal cost, relative to traditional destructive harvesting. As unrepresentative allometric relationships contribute a substantial proportion of uncertainty in landscape-scale biomass and carbon distributions, this approach aids in improving these estimates for more effective global forest carbon management. 


\subsection{Appendix}

\section{Model Parameters}

Table 2.4 shows the regression parameters used in the models to estimate foliage and wood mass of individual branches. Table 2.5 shows calibration parameters for $O H M_{\mathrm{P}}$.

Table 2.4: Regression parameter values (standard error) for estimating foliage (Equation 1; $\mathrm{n}=90$ ) and wood (Equation 2; $\mathrm{n}=89$ ) wet mass for branches.

\begin{tabular}{lccc}
\hline \hline Equation & $\beta_{0}$ & $\beta_{1}$ & $\beta_{2}$ \\
\hline 1 & $3.9583(0.1181)$ & $1.9426(0.1377)$ & \\
\hline 2 & $4.1965(0.0699)$ & $1.9184(0.1031)$ & $0.7696(0.1418)$ \\
\hline
\end{tabular}

Table 2.5: Calibration parameter values (standard error) for the OHM algorithm $\left(O H M_{\mathrm{P}}\right)$.

\begin{tabular}{lccc}
\hline \hline Model & Parameter & Coefficient & $\mathrm{p}$ \\
\hline$O H M_{\mathrm{P}}$ & $\mathrm{a}$ & $10.06(1.04)$ & $<0.001$ \\
& $\mathrm{~b}$ & $0.46(0.01)$ & $<0.001$ \\
\hline
\end{tabular}

\section{Preprocessing of TLS Data}

Phase-based TLS units suffer from stray points in complex vegetation caused by ambiguous distance measurements in the return signal that make noiseless scan data difficult to achieve without filtering. Scans that contain fine branches or needles are particularly vulnerable to this type of noise. We filtered the tree scans using a custom stray point filter and statistical outlier removal (Figure 2.10). The stray point filter relies on three parameters: allocation threshold, distance threshold, and grid size (Newnham et al., 2012). The grid size defines the area to include in the filtering procedure. If a percentage of points, given by the allocation threshold fall outside the distance threshold, they are removed from the scan data. A statistical outlier removal filter was used that is based on an algorithm provided in the open source Point Cloud Library (Rusu \& Cousins, 2011). This algorithm 


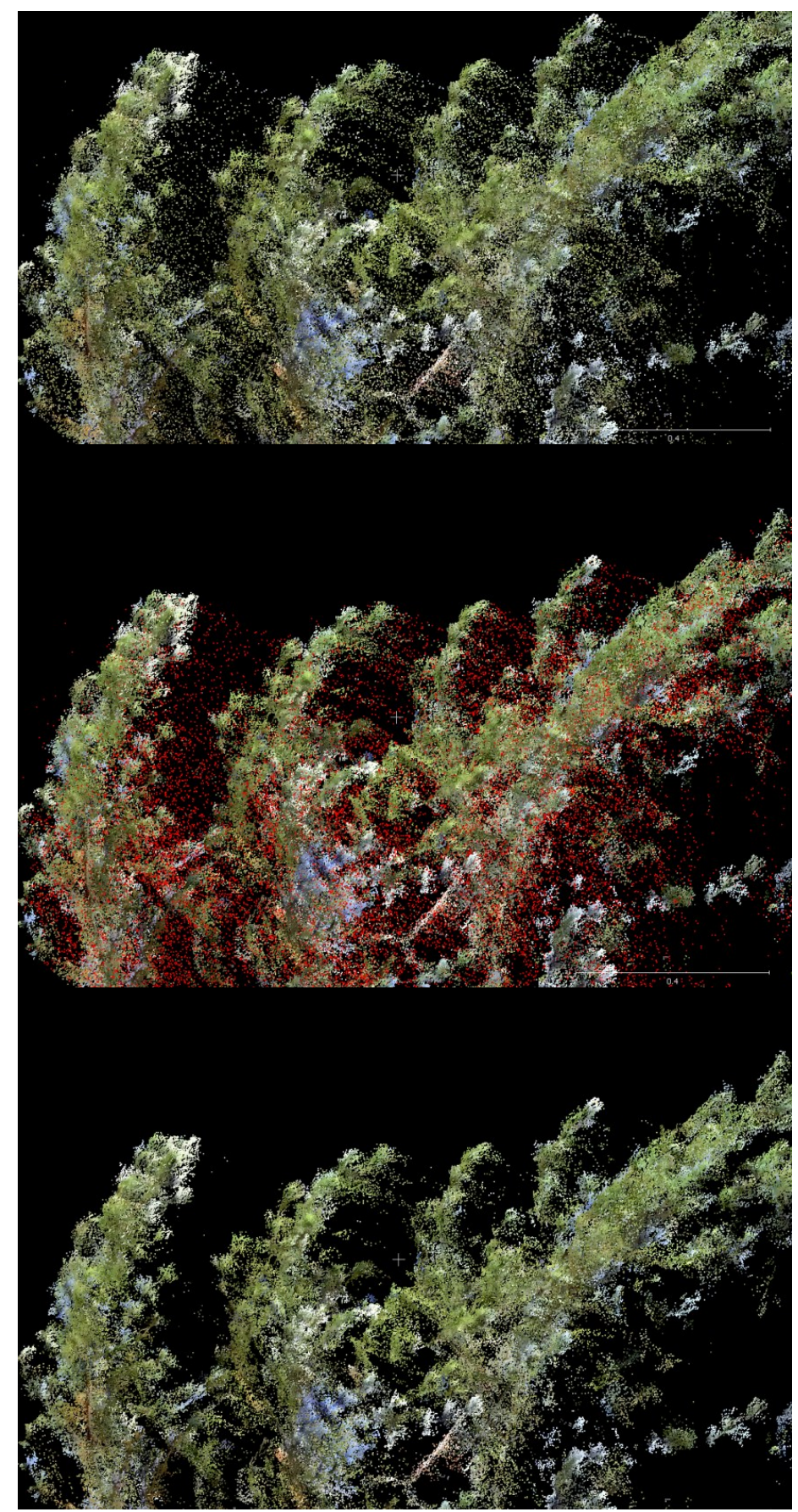

Figure 2.10: Example of noise removal procedure. The raw point cloud (top) is denoised with stay point removal and statistical outlier removal. Noise points (middle, in red) are removed for a clean point cloud (bottom). 
functions by analyzing the neighborhood around an individual scan point for the mean distance to all surrounding points, comparing this distribution to a Gaussian distribution, with a mean and standard deviation, and removing all points whose mean distances deem them as outliers.

The use of a phase-based scanner resulted in significant noise near the central bole, so the point cloud was manually segmented the point cloud, rather than using automated methods, to differentiate between the trunk and branch points. The segment tool was used in CloudCompare to remove all branch points and expose the trunk. Often times, large portions of the central trunk were lost from the scan data due to occlusion effects. In situations such as this, the point cloud was roughly segmented leaving as many points attributed to the central bole as possible. In the case of trunk bifrication or complex branching structure on larger trees, the main large branches underwent the same segmentation process. Each tree took 10-20 minutes to preprocess. As this is a manual process, it is dependent on user skill, but the segmentation of branch from trunk points is an intuitive and relatively simple step. Finally, each component was exported in ASCII format in order to be used in the volumetric modeling process.

\section{Vertical Buffer for Reducing Occlusion}

Terrestrial LiDAR datasets that have incomplete coverage of returns from the trunk section due to occlusion effects require an alternative method to help reduce gaps in data. Since this algorithm uses a convex hull method, the point set will always be enclosed, regardless of the shape of the sliced trunk in the point cloud. This function is of great benefit because area, and thus volume, can be computed at any trunk section, assuming it contains 3 or more points. However, in situations where point coverage is incomplete and large sections of the trunk are unmeasured there can be significant volume underestimation, because the polygon created from the convex hull process will simply connect the next closest and outermost point, which may not be representative of the reality of the trunk shape. A convex hull method of gap-filling has been used in a modeling algorithm applied to mangrove trees (Olagoke et al., 2016), however the significant gaps present from occluded trunk points in our data would have produced unacceptable volume errors. 
In order to reduce this source of error we implement a buffering procedure at each successive slice that the convex hull was computed for (Figure 2.11). The buffer functions by including all points above and below the slice being modeled, increasing the likelihood of the outer trunk points being included in the final model. The distance of the buffer can be varied according to the coverage of LiDAR returns and level of occlusion present on the individual tree. Increasing the buffer produces a smoothing effect on the final model that is ideal in circumstances of occlusion, but may alter the accuracy of the representation of trunk shape. This buffering process has the added benefit of increasing the likelihood that the modeling procedure will complete at each section because sections with less than 3 points will likely increase in point density. The option for buffering at each successive trunk slice is essential for providing the most complete and representative volumetric model of the central bole of the tree.

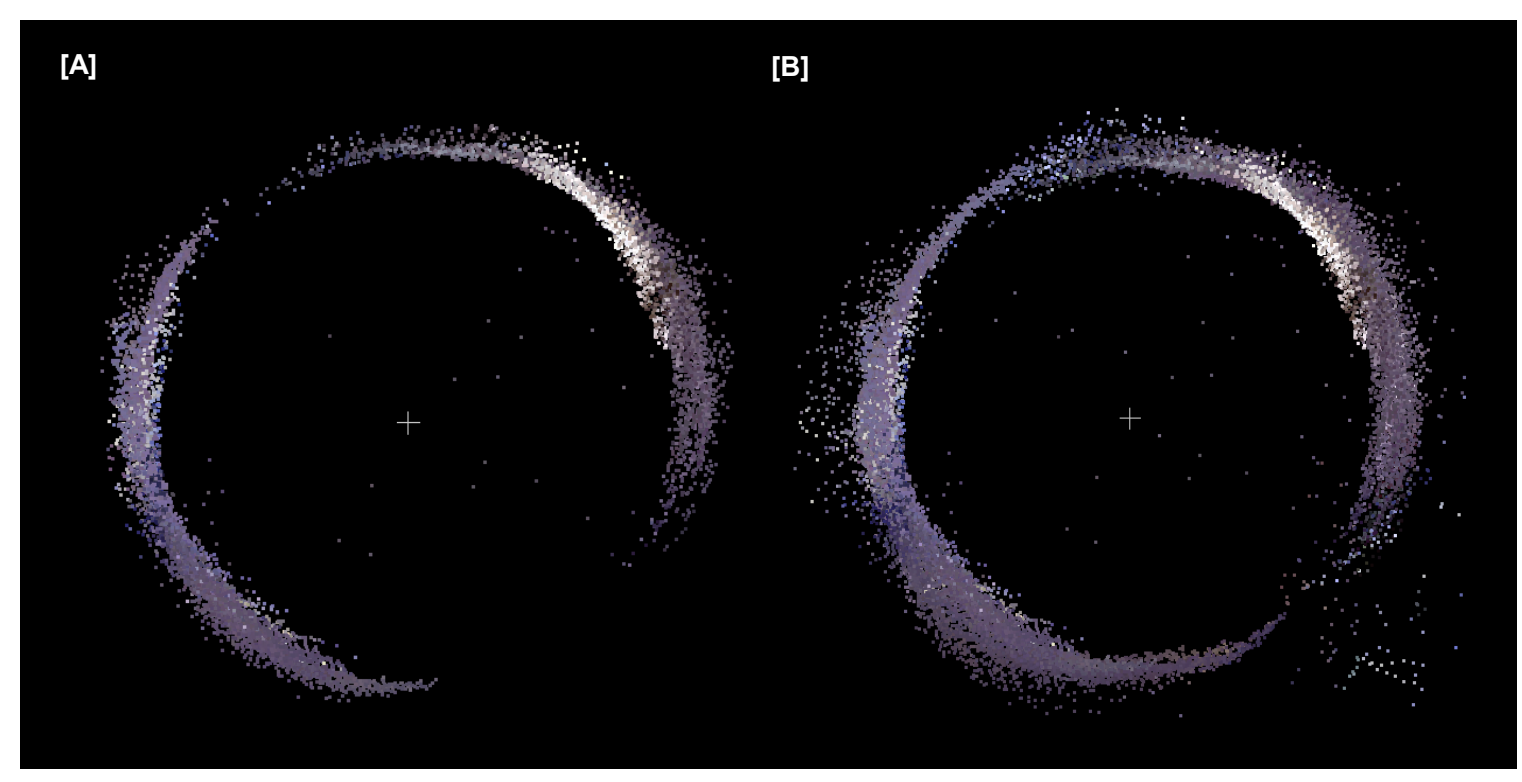

Figure 2.11: Comparison of $[\mathrm{A}]$ trunk point cloud slice and [B] trunk point cloud slice with vertical buffer. Prominent gaps in the lower right of the point cloud due to occlusion are filled using this method. 


\section{Convex Hull Peeling}

The final modeled fit of the convex hull peeling operation outperformed circle fitting (Figure 2.12). RMSE increases when voxelization is used to reduce computational time, but the OHM algorithm still performs well (Figure 2.13).

A
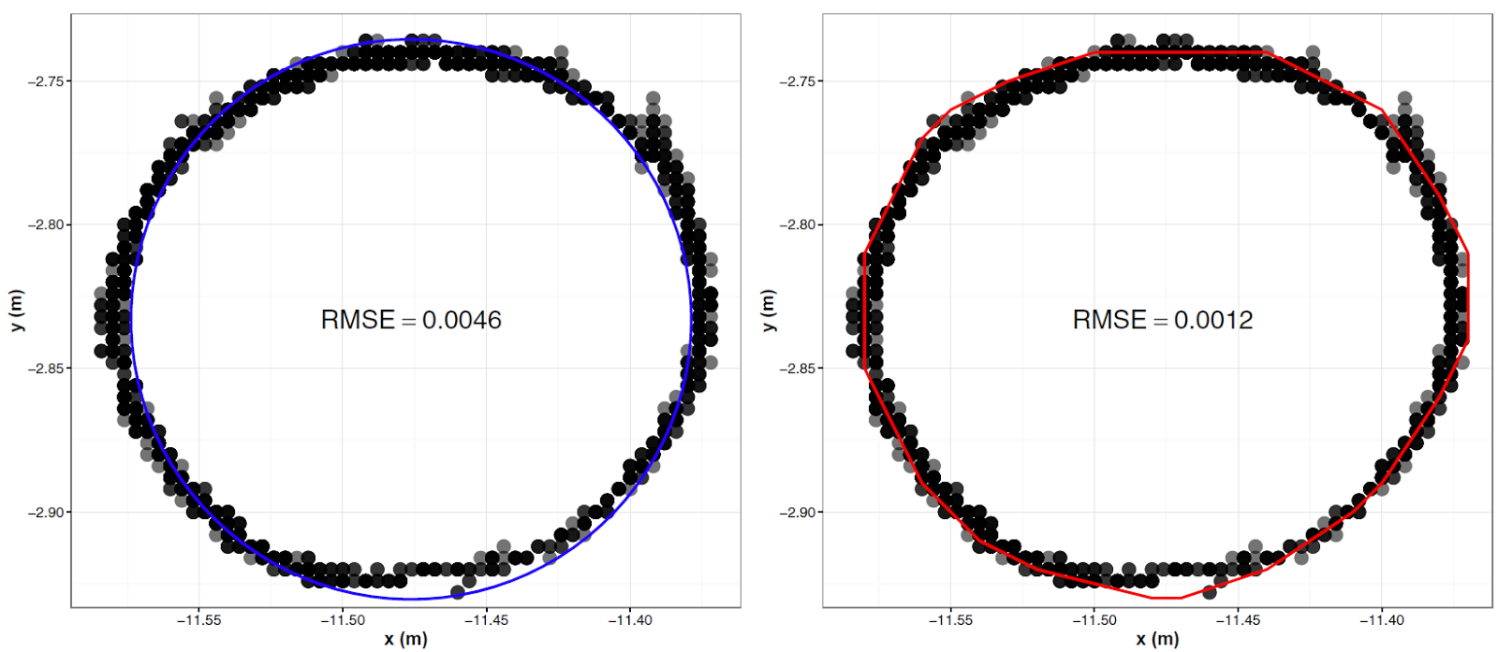

Figure 2.12: Comparison of $[\mathrm{A}]$ least squares circle fit to [B] convex hull peeling as implemented in the OHM algorithm. The reduction in RMSE (m) is substantial on this low noise trunk slice. Points are slightly transparent in order to show locations of high return density (darker). 
A

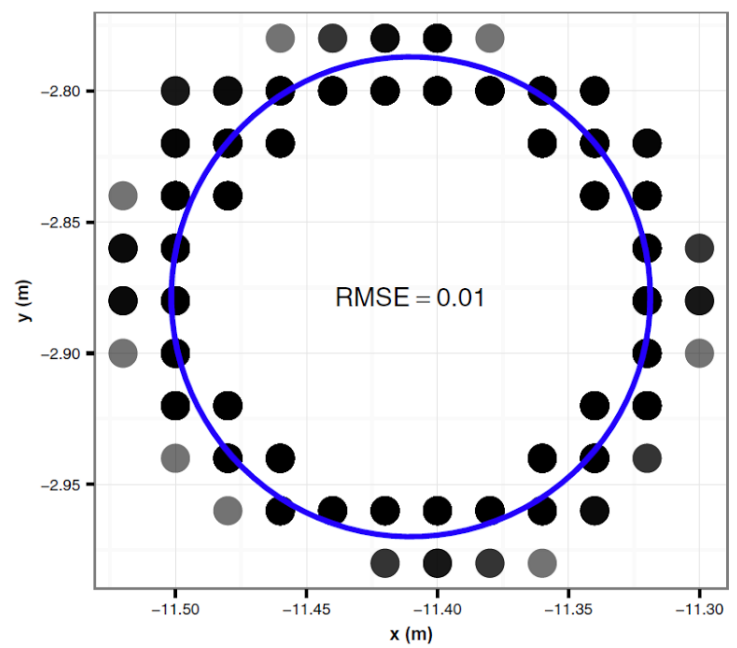

B

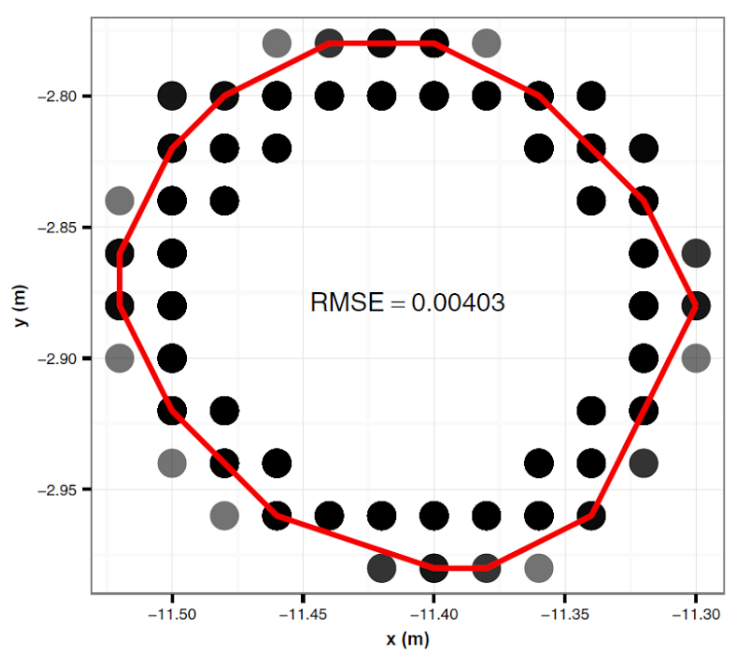

Figure 2.13: Comparison of [A] least squares circle fit to [B] convex hull peeling as implemented in the OHM algorithm. This example includes the voxelization procedure for reduced computation time. Note: RMSE $(\mathrm{m})$ is higher when the point cloud has been voxelized. Points are slightly transparent in order to show locations of high return density (darker). 
Table 2.6: Comparison of absolute difference in RMSE (\%) with an average density value (0.38) versus measured average individual tree density. Negative differences indicate improvement in RMSE.

\begin{tabular}{|c|c|c|c|c|}
\hline Component & Status & $\begin{array}{c}\text { Average Density } \\
\text { RMSE (\%) }\end{array}$ & $\begin{array}{c}\text { Measured Density } \\
\text { RMSE }(\%)\end{array}$ & RMSE $_{\text {MEAS }}-\mathrm{RMSE}_{\mathrm{AVE}}(\%)$ \\
\hline \multirow{3}{*}{ Total Biomass } & Whole & 11.1 & 12.9 & 1.8 \\
\hline & Live & 15.2 & 16.6 & 1.4 \\
\hline & Dead & 3.3 & 6.5 & 3.2 \\
\hline \multirow[t]{3}{*}{ Woody Biomass } & Whole & 10.2 & 12.1 & 1.9 \\
\hline & Live & 14.4 & 16.1 & 1.7 \\
\hline & Dead & 3.2 & 6.4 & 3.2 \\
\hline \multirow[t]{3}{*}{ Trunk Biomass } & Whole & 8.4 & 7.0 & -1.4 \\
\hline & Live & 8.9 & 9.5 & 0.6 \\
\hline & Dead & 7.5 & 3.3 & -4.2 \\
\hline \multirow[t]{3}{*}{ Branch Biomass } & Whole & 49.4 & 49.4 & 0.0 \\
\hline & Live & 47.1 & 46.5 & -0.6 \\
\hline & Dead & 54.9 & 56.7 & 1.8 \\
\hline Branch and Needle Biomass & Live & 41.8 & 41.6 & -0.2 \\
\hline Needle Biomass & Live & 38.9 & 39.0 & 0.1 \\
\hline
\end{tabular}

\section{Density Effects}

We evaluated the effect of density on our biomass estimates. Trunk biomass estimates were improved when using measured biomass, while most other component estimates showed no improvement.(Table 2.6; Figure 2.14).

\section{Comparison to QSMs}

Quantitative surface models (QSMs) do not perform well with the high noise data common to phase-shift TLS when scanning needleleaf trees. We estimated the volume and biomass of the trees in this study using a QSM approach implemented in the SimpleTree plugin within Computree (Hackenberg et al., 2015b). We found many low noise, dead samples performed well, but higher noise samples significantly overestimated biomass (Figure 2.15). In one case, the QSM approach overestimated biomass by more than $250 \%$. Low noise samples are clearly modeled well, but large cylinders become common in the high noise crown, leading to unrealistic biomass estimates. One other finding from using the QSM method in 


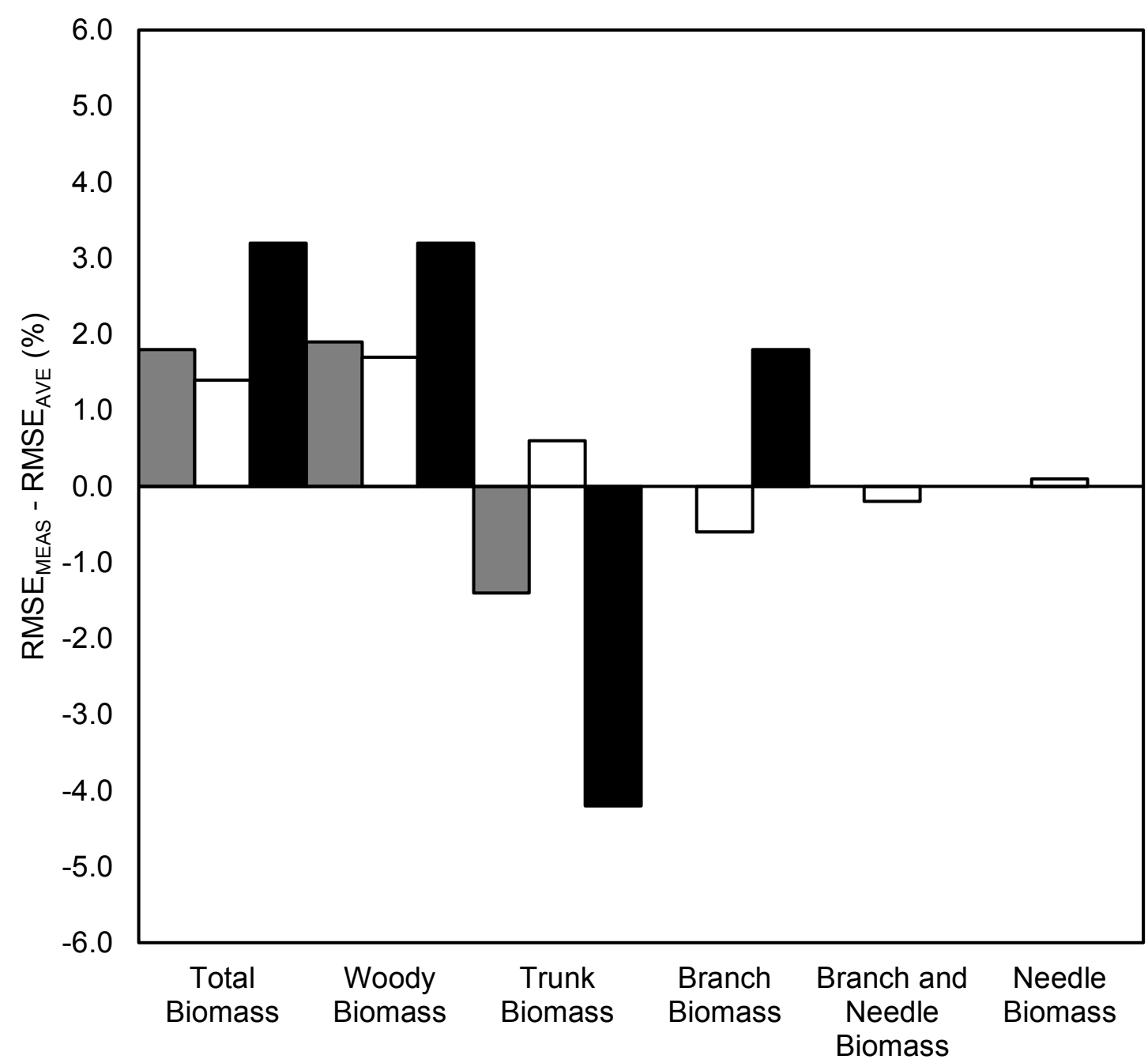

Figure 2.14: Comparison of absolute difference in RMSE (\%) with an average density value (0.38) versus measured average individual tree density for live and dead (grey), live (white), and dead (black). Negative differences indicate improvement in RMSE. 
comparison to OHM and branch voxelization was the increase in computational time to 7.5 hours (21 minutes per tree on average). We modeled the isolated trunk point cloud to evaluate the location of the main errors in biomass estimation. Trunk biomass was estimated with accuracy approaching that of the OHM algorithm when the QSMs were run on the trunk point cloud (Figure 2.16). Our method of estimating branch and needle biomass could easily be integrated into a QSM approach by isolating the trunk point cloud and feeding the trunk volume information into the branch estimation algorithm. Future developments of the branch biomass algorithm will be capable of using QSM input data for needleleaf trees. 


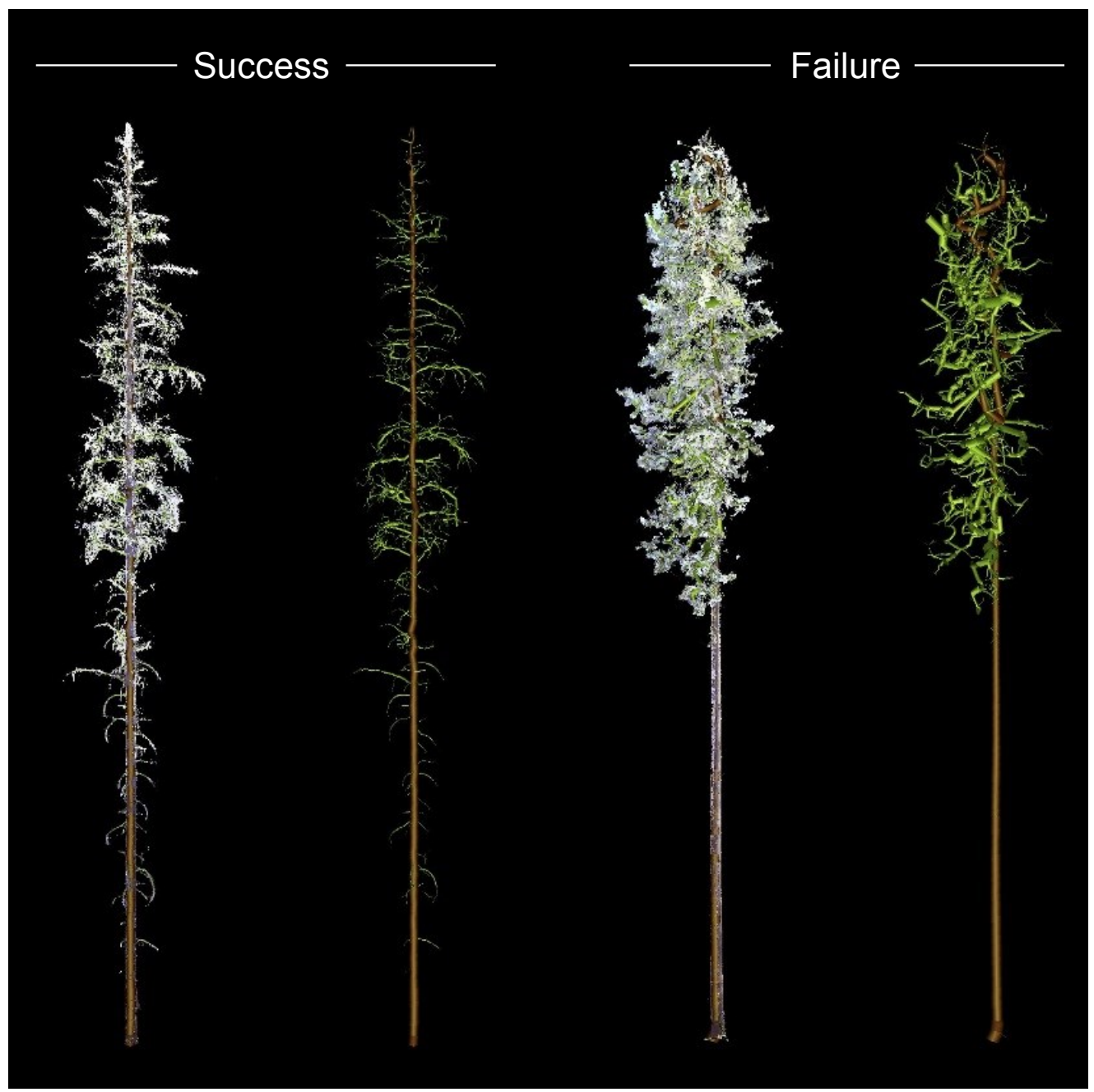

Figure 2.15: Example of successful and unsuccessful cylinder fitting of two different tree point clouds. The successful scenario (left) was completed on a dead individual with low noise and branches are clearly accurately represented. The unsuccessful scenario (right) was completed on a living tree with dense foliage and high point cloud noise, resulting in unrealistic cylinder sizes withing the crown. 

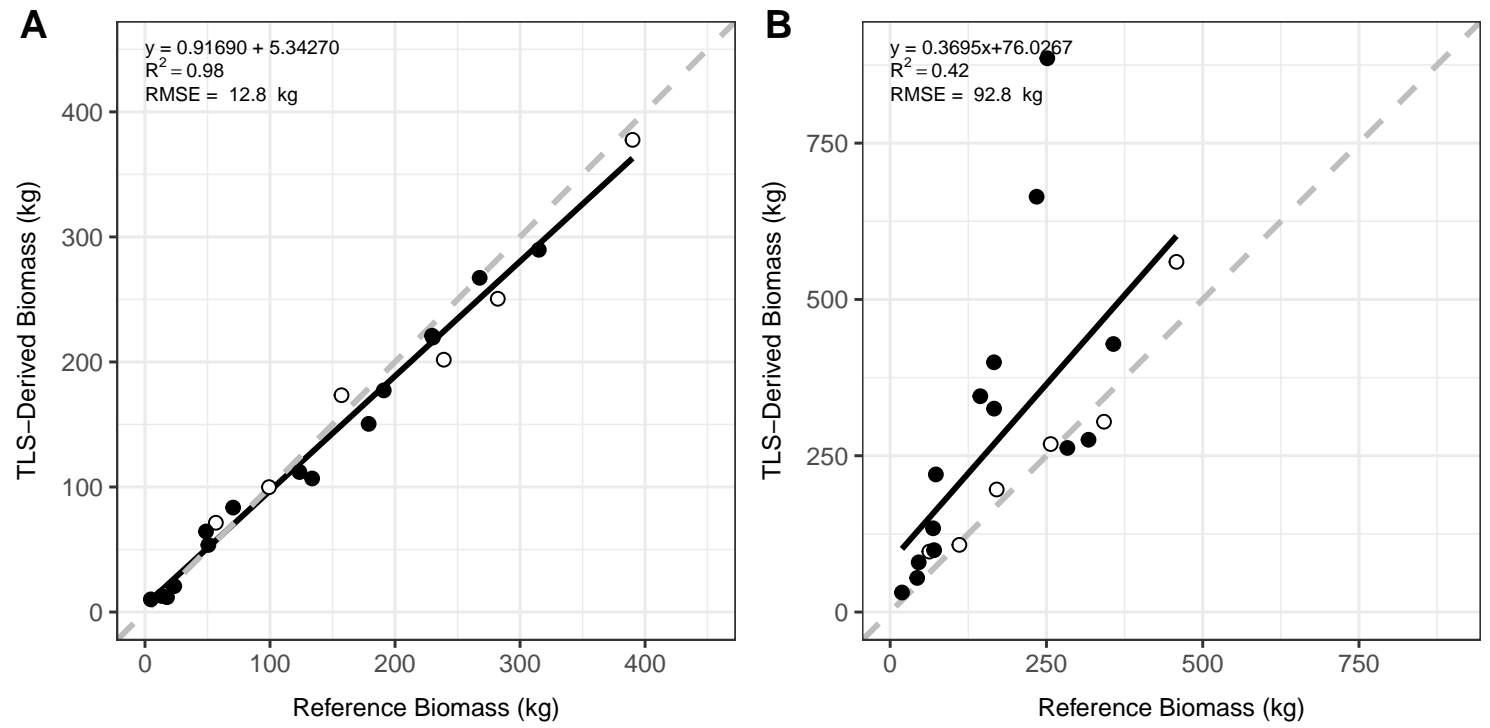

Figure 2.16: Non-destructive estimates of biomass using a QSM approach on the (a) segmented trunk point cloud and (b) the entire unsegmented point cloud for living (black) and dead (open-circles) trees. 


\section{References}

Baccini, A., Goetz, SJ, Walker, WS, Laporte, NT, Sun, M, Sulla-Menashe, D, Hackler, J, Beck, PSA, Dubayah, R, Friedl, MA, \& others. 2012. Estimated carbon dioxide emissions from tropical deforestation improved by carbon-density maps. Nature Climate Change, 2(3), 182-185.

Bienert, A., Scheller, S., Keane, E., Mullooly, G., \& Mohan, F. 2006. Application of terrestrial laser scanners for the determination of forest inventory parameters. International Archives of Photogrammetry, Remote Sensing and Spatial Information Sciences, 36(Part 5).

Bienert, A., Hess, C., Maas, H.-G., \& von Oheimb, G. 2014. A voxel-based technique to estimate the volume of trees from terrestrial laser scanner data. ISPRS - International Archives of the Photogrammetry, Remote Sensing and Spatial Information Sciences, XL-5(June), 101-106.

Brown, S. 1997. Estimating biomass and biomass change of tropical forests. A primer.

Calders, Kim, Newnham, Glenn, Burt, Andrew, Murphy, Simon, Raumonen, Pasi, Herold, Martin, Culvenor, Darius, Avitabile, Valerio, Disney, Mathias, Armston, John, \& Kaasalainen, Mikko. 2015. Nondestructive estimates of above-ground biomass using terrestrial laser scanning. Methods in Ecology and Evolution, 6(2), 198-208.

Chave, J., Andalo, C., Brown, S., Cairns, M. A., Chambers, J. Q., Eamus, D., Flster, H., Fromard, F., Higuchi, N., Kira, T., Lescure, J.-P., Nelson, B. W., Ogawa, H., Puig, H., Rira, B., \& Yamakura, T. 2005. Tree allometry and 
improved estimation of carbon stocks and balance in tropical forests. Oecologia, 145(1), 87-99.

Chave, Jerome, Condit, Richard, Aguilar, Salomon, Hernandez, Andres, Lao, Suzanne, \& Perez, Rolando. 2004. Error propagation and scaling for tropical forest biomass estimates. Philosophical Transactions of the Royal Society B: Biological Sciences, 359(1443), 409-420.

Chazelle, B. 1985. On the convex layers of a planar set. IEEE Transactions on Information Theory, 31(4), 509-517.

Chojnacky, D. C., Heath, L. S., \& Jenkins, Jennifer C. 2014. Updated generalized biomass equations for North American tree species. Forestry, 87(1), 129-151.

CloudCompare. 2015. GPL Software.

Coope, I.D. 1993. Circle fitting by linear and nonlinear least squares. Journal of Optimization Theory and Applications, 76(2), 381-388.

Côté, Jean-Franois, Widlowski, Jean-Luc, Fournier, Richard A., \& Verstraete, Michel M. 2009. The structural and radiative consistency of three-dimensional tree reconstructions from terrestrial lidar. Remote Sensing of Environment, 113(5), 1067-1081.

Côté, Jean-Franois, Fournier, Richard A., \& Egli, Richard. 2011. An architectural model of trees to estimate forest structural attributes using terrestrial LiDAR. Environmental Modelling \& Software, 26(6), 761-777.

Danson, F. Mark, Hetherington, David, Morsdorf, Felix, Koetz, Benjamin, \& Allgower, Britta. 2007. Forest Canopy Gap Fraction From Terrestrial Laser Scanning. IEEE Geoscience and Remote Sensing Letters, 4(1), 157-160.

Dassot, Mathieu, Constant, Thiry, \& Fournier, Meriem. 2011. The use of terrestrial LiDAR technology in forest science: application fields, benefits and challenges. Annals of Forest Science, 68(5), 959-974. 
de Miguel, Sergio, Pukkala, Timo, Assaf, Nabil, \& Shater, Zuheir. 2014. Intraspecific differences in allometric equations for aboveground biomass of eastern Mediterranean Pinus brutia. Annals of Forest Science, 71(1), 101-112.

Eddy, William F. 1977. A New Convex Hull Algorithm for Planar Sets. ACM Trans. Math. Softw., 3(4), 398-403.

Falkowski, Michael J., Smith, Alistair MS, Hudak, Andrew T., Gessler, Paul E., Vierling, Lee A., \& Crookston, Nicholas L. 2006. Automated estimation of individual conifer tree height and crown diameter via two-dimensional spatial wavelet analysis of lidar data. Canadian Journal of Remote Sensing, 32(2), $153-161$.

Feldpausch, T. R., Banin, L., Phillips, O. L., Baker, T. R., Lewis, S. L., Quesada, C. A., Affum-Baffoe, K., Arets, E. J. M. M., Berry, N. J., Bird, M., Brondizio, E. S., de Camargo, P., Chave, J., Djagbletey, G., Domingues, T. F., Drescher, M., Fearnside, P. M., Franca, M. B., Fyllas, N. M., Lopez-Gonzalez, G., Hladik, A., Higuchi, N., Hunter, M. O., Iida, Y., Salim, K. A., Kassim, A. R., Keller, M., Kemp, J., King, D. A., Lovett, J. C., Marimon, B. S., Marimon-Junior, B. H., Lenza, E., Marshall, A. R., Metcalfe, D. J., Mitchard, E. T. A., Moran, E. F., Nelson, B. W., Nilus, R., Nogueira, E. M., Palace, M., Patino, S., Peh, K. S.-H., Raventos, M. T., Reitsma, J. M., Saiz, G., Schrodt, F., Sonke, B., Taedoumg, H. E., Tan, S., White, L., Woell, H., \& Lloyd, J. 2011. Height-diameter allometry of tropical forest trees. Biogeosciences, 8(5), 1081-1106.

Griebel, Anne, Bennett, Lauren T., Culvenor, Darius S., Newnham, Glenn J., \& Arndt, Stefan K. 2015. Reliability and limitations of a novel terrestrial laser scanner for daily monitoring of forest canopy dynamics. Remote Sensing of Environment, 166(Sept.), 205-213.

Hackenberg, Jan, Morhart, Christopher, Sheppard, Jonathan, Spiecker, Heinrich, \& Disney, Mathias. 2014. Highly Accurate Tree Models Derived from Terrestrial Laser Scan Data: A Method Description. Forests, 5(5), 1069-1105.

Hackenberg, Jan, Wassenberg, Marc, Spiecker, Heinrich, \& Sun, Dongjing. 2015a. 
Non destructive method for biomass prediction combining TLS derived tree volume and wood density. Forests, 6(4), 1274-1300.

Hackenberg, Jan, Spiecker, Heinrich, Calders, Kim, Disney, Mathias, \& Raumonen, Pasi. 2015b. SimpleTree-An Efficient Open Source Tool to Build Tree Models from TLS Clouds. Forests, 6(11), 4245-4294.

Henning, Jason G., \& Radtke, Philip J. 2006. Detailed stem measurements of standing trees from ground-based scanning lidar. Forest Science, 52(1), 67-80.

Hopkinson, Chris, Chasmer, Laura, Young-Pow, Colin, \& Treitz, Paul. 2004. Assessing forest metrics with a ground-based scanning lidar. Canadian Journal of Forest Research, 34(3), 573-583.

Hosoi, Fumiki, Nakai, Yohei, \& Omasa, Kenji. 2013. 3-D voxel-based solid modeling of a broad-leaved tree for accurate volume estimation using portable scanning lidar. ISPRS Journal of Photogrammetry and Remote Sensing, 82(Aug.), 41-48.

Houghton, R. A., Hall, Forrest, \& Goetz, Scott J. 2009. Importance of biomass in the global carbon cycle. Journal of Geophysical Research-Biogeosciences, 114(Sept.), G00E03.

Jenkins, J. C., Chojnacky, David C., Heath, Linda S., \& Birdsey, Richard A. 2003. National-scale biomass estimators for United States tree species. Forest Science, 49(1), 12-35.

Jupp, David L. B., Culvenor, D.S., Lovell, J.L., Newnham, G.J., Strahler, A.H., \& Woodcock, C.E. 2008. Estimating forest LAI profiles and structural parameters using a ground-based laser called Echidna. Tree Physiology, 29(2), 171-181.

Kershaw, J. A., \& Maguire, D. A. 1995. Crown structure in Western hemlock, Douglas-fir, and grand fir in western Washington: trends in branch-level mass and leaf area. Can J For Res, 25.

Lefsky, Michael, \& McHale, Melissa. 2008. Volume estimates of trees with complex architecture from terrestrial laser scanning. Journal of Applied Remote Sensing, 2, 023521 . 
Liang, Xinlian, Kankare, Ville, Hyypp, Juha, Wang, Yunsheng, Kukko, Antero, Haggrn, Henrik, Yu, Xiaowei, Kaartinen, Harri, Jaakkola, Anttoni, Guan, Fengying, Holopainen, Markus, \& Vastaranta, Mikko. 2016. Terrestrial laser scanning in forest inventories. ISPRS Journal of Photogrammetry and Remote Sensing, Feb.

Lovell, J. L., Jupp, D. L. B., Culvenor, D. S., \& Coops, N. C. 2003. Using airborne and ground-based ranging lidar to measure canopy structure in Australian forests. Canadian Journal of Remote Sensing, 29(5), 607-622.

Lovell, J. L., Jupp, D. L. B., Newnham, G. J., \& Culvenor, D. S. 2011. Measuring tree stem diameters using intensity profiles from ground-based scanning lidar from a fixed viewpoint. Isprs Journal of Photogrammetry and Remote Sensing, 66(1), 46-55.

Maas, H.G., Bienert, A., Scheller, S., \& Keane, E. 2008. Automatic forest inventory parameter determination from terrestrial laser scanner data. International Journal of Remote Sensing, 29(5), 1579-1593.

Mitchard, Edward T. A., Feldpausch, Ted R., Brienen, Roel J. W., LopezGonzalez, Gabriela, Monteagudo, Abel, Baker, Timothy R., Lewis, Simon L., Lloyd, Jon, Quesada, Carlos A., Gloor, Manuel, ter Steege, Hans, Meir, Patrick, Alvarez, Esteban, Araujo-Murakami, Alejandro, Aragao, Luiz E. O. C., Arroyo, Luzmila, Aymard, Gerardo, Banki, Olaf, Bonal, Damien, Brown, Sandra, Brown, Foster I., Ceron, Carlos E., Chama Moscoso, Victor, Chave, Jerome, Comiskey, James A., Cornejo, Fernando, Corrales Medina, Massiel, Da Costa, Lola, Costa, Flavia R. C., Di Fiore, Anthony, Domingues, Tomas F., Erwin, Terry L., Frederickson, Todd, Higuchi, Niro, Honorio Coronado, Euridice N., Killeen, Tim J., Laurance, William F., Levis, Carolina, Magnusson, William E., Marimon, Beatriz S., Marimon Junior, Ben Hur, Mendoza Polo, Irina, Mishra, Piyush, Nascimento, Marcelo T., Neill, David, Nunez Vargas, Mario P., Palacios, Walter A., Parada, Alexander, Pardo Molina, Guido, Pena-Claros, Marielos, Pitman, Nigel, Peres, Carlos A., Poorter, Lourens, Prieto, Adriana, RamirezAngulo, Hirma, Restrepo Correa, Zorayda, Roopsind, Anand, Roucoux, Katherine H., Rudas, Agustin, Salomao, Rafael P., Schietti, Juliana, Silveira, Mar- 
cos, de Souza, Priscila F., Steininger, Marc K., Stropp, Juliana, Terborgh, John, Thomas, Raquel, Toledo, Marisol, Torres-Lezama, Armando, van Andel, Tinde R., van der Heijden, Geertje M. F., Vieira, Ima C. G., Vieira, Simone, Vilanova-Torre, Emilio, Vos, Vincent A., Wang, Ophelia, Zartman, Charles E., Malhi, Yadvinder, \& Phillips, Oliver L. 2014. Markedly divergent estimates of Amazon forest carbon density from ground plots and satellites. Global Ecology and Biogeography, 23(8), 935-946.

Newnham, Glenn, Armston, John, Muir, Jasmine, Goodwin, Nicholas, Tindall, D., Culvenor, D., Pschel, P., Nystrm, M., \& Johansen, K. 2012. Evaluation of terrestrial laser scanners for measuring vegetation structure. Australia: CSIRO.

Olagoke, Adewole, Proisy, Christophe, Fret, Jean-Baptiste, Blanchard, Elodie, Fromard, Franois, Mehlig, Ulf, de Menezes, Moirah Machado, dos Santos, Valdenira Ferreira, \& Berger, Uta. 2016. Extended biomass allometric equations for large mangrove trees from terrestrial LiDAR data. Trees, 30(3), 935-947.

Omasa, Kenji, Hosoi, Fumiki, \& Konishi, A. 2006. 3D lidar imaging for detecting and understanding plant responses and canopy structure. Journal of Experimental Botany, 58(4), 881-898.

Picard, Nicolas, Saint-Andre, Laurent, \& Henry, Matieu. 2012. Manual for building tree volume and biomass allometric equations: from field measurement to prediction. Food and Agricultural Organization of the United Nations and Centre de Coopration Internationale en Recherche Agronomique pour le Dveloppement.

Poudel, Krishna P., Temesgen, Hailemariam, \& Gray, Andrew N. 2015. Evaluation of sampling strategies to estimate crown biomass. Forest Ecosystems, 2(1), 1.

Raumonen, Pasi, Kaasalainen, Mikko, kerblom, Markku, Kaasalainen, Sanna, Kaartinen, Harri, Vastaranta, Mikko, Holopainen, Markus, Disney, Mathias, \& Lewis, Philip. 2013. Fast Automatic Precision Tree Models from Terrestrial Laser Scanner Data. Remote Sensing, 5(2), 491-520.

Rusu, Radu Bogdan, \& Cousins, Steve. 2011. 3d is here: Point cloud library (pcl). Pages 1-4 of: Robotics and Automation (ICRA), 2011 IEEE International Conference on. IEEE. 
Saatchi, Sassan, Harris, Nancy L., Brown, Sandra, Lefsky, Michael, Mitchard, Edward T. A., Salas, William, Zutta, Brian R., Buermann, Wolfgang, Lewis, Simon L., Hagen, Stephen, Petrova, Silvia, White, Lee, Silman, Miles, \& Morel, Alexandra. 2011. Benchmark map of forest carbon stocks in tropical regions across three continents. Proceedings of the National Academy of Sciences of the United States of America, 108(24), 9899-9904.

SCENE (version 5.4.4.41689). 2015. FARO Technologies Inc.

Seidel, Dominik, Beyer, Friderike, Hertel, Dietrich, Fleck, Stefan, \& Leuschner, Christoph. 2011. 3D-laser scanning: A non-destructive method for studying above- ground biomass and growth of juvenile trees. Agricultural and Forest Meteorology, 151(10), 1305 - 1311.

Tansey, K., Selmes, N., Anstee, A., Tate, N. J., \& Denniss, A. 2009. Estimating tree and stand variables in a Corsican Pine woodland from terrestrial laser scanner data. International Journal of Remote Sensing, 30(19), 5195-5209.

Temesgen, Hailemariam, Monleon, Vicente, Weiskittel, Aaron, \& Wilson, Duncan. 2011. Sampling strategies for efficient estimation of tree foliage biomass. Forest Science, 57(2), 153-163.

TerMikaelian, M. T., \& Korzukhin, M. D. 1997. Biomass equations for sixty-five North American tree species. Forest Ecology and Management, 97(1), 1-24.

USDA Forest Service. 2013. Forest Insect and Disease Aerial Survey Data, Rocky Mountain Region. Tech. rept.

West, Geoffrey B., Brown, James H., \& Enquist, Brian J. 1999. A general model for the structure and allometry of plant vascular systems. Nature, 400(6745), $664-667$.

Williamson, G. B., \& Wiemann, M. C. 2010. Measuring wood specific gravity...Correctly. American Journal of Botany, 97(3), 519-524.

Yao, Tian, Yang, Xiaoyuan, Zhao, Feng, Wang, Zhuosen, Zhang, Qingling, Jupp, David, Lovell, Jenny, Culvenor, Darius, Newnham, Glenn, Ni-Meister, Wenge, 
Schaaf, Crystal, Woodcock, Curtis, Wang, Jindi, Li, Xiaowen, \& Strahler, Alan. 2011. Measuring forest structure and biomass in New England forest stands using Echidna ground-based lidar. Remote Sensing of Environment, 115(11), 2965-2974.

Yu, Xiaowei, Liang, Xinlian, Hyyppa, Juha, Kankare, Ville, Vastaranta, Mikko, \& Holopainen, Markus. 2013. Stem biomass estimation based on stem reconstruction from terrestrial laser scanning point clouds. Remote Sensing Letters, 4(4), 344-353.

Zhao, Feng, Yang, Xiaoyuan, Schull, Mitchell A., Romn-Coln, Miguel O., Yao, Tian, Wang, Zhuosen, Zhang, Qingling, Jupp, David L. B., Lovell, Jenny L., Culvenor, Darius S., Newnham, Glenn J., Richardson, Andrew D., Ni-Meister, Wenge, Schaaf, Crystal L., Woodcock, Curtis E., \& Strahler, Alan H. 2011. Measuring effective leaf area index, foliage profile, and stand height in New England forest stands using a full-waveform ground-based lidar. Remote Sensing of Environment, 115(11), 2954 - 2964. 


\section{Chapter 3}

Implications of Applying Non-Destructive Local Allometry

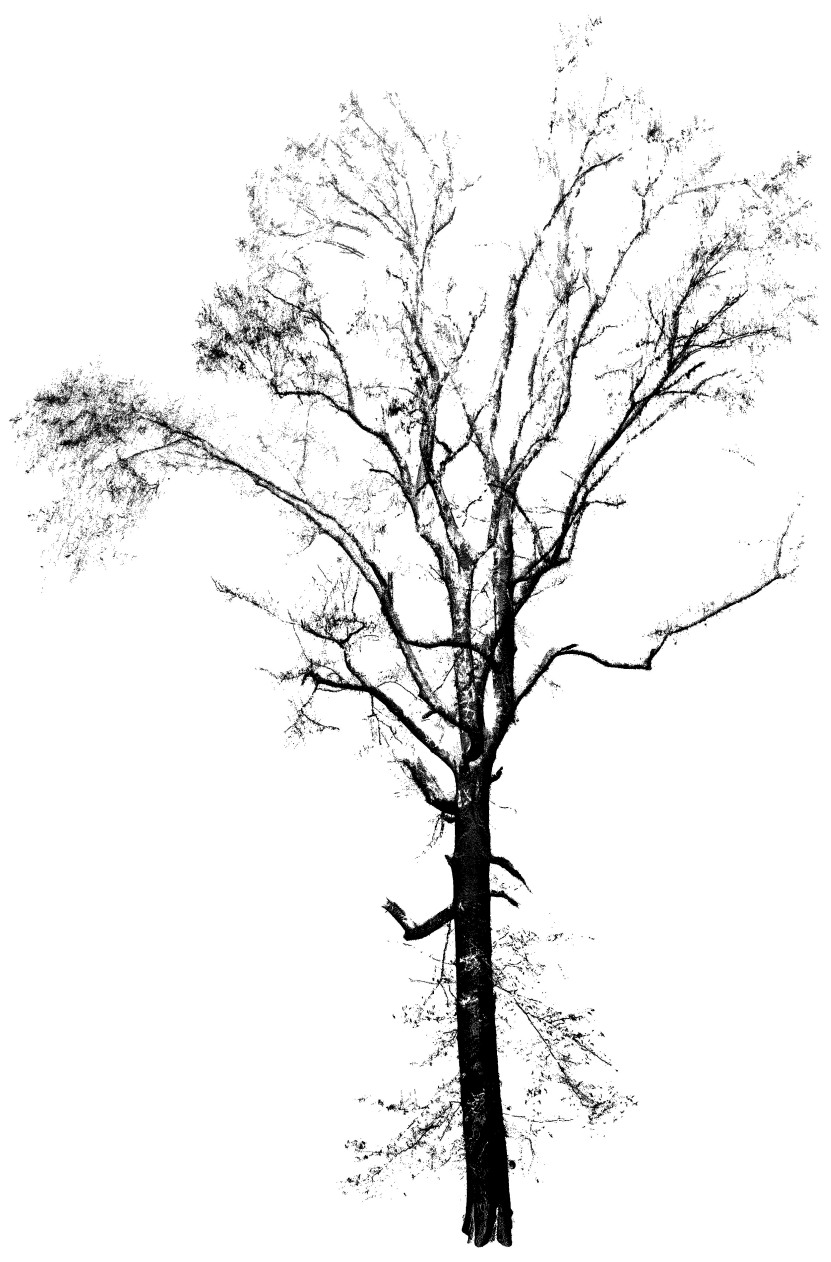




\section{Abstract}

Estimates of the quantity and spatial distribution of global forest carbon are built on the assumption that regional- or national-scale allometry accurately captures growth form across the wide spectrum of plant size. Allometry is painstaking to create: trees must be cut, dried, and weighed over the span of months. This bottleneck has left most equations low in sample size and without the largest trees, which can contain around $40 \%$ of forest carbon. Terrestrial laser scanning can potentially increase the range and sample size of allometric equations through non-destructive biomass estimation and must be evaluated in this context. We used TLS to virtually reconstruct 329 trees ranging from 4 to $123 \mathrm{~cm}$ diameter. Three-dimensional tree models were the basis for 34 local allometric relationships for comparison to the Jenkins et al. (2003) and Chojnacky et al. (2014) equations. Overall, TLS allometry had lower RMSE and predicted higher biomass compared to the equivalent national equations. We evaluated site-wide allometry for errors from insufficient sample-size and diameter range. We found allometric equations did not stabilize to a consistent set of parameters until 100-200 samples were reached, suggesting many current biomass equations may be inadequate. This work highlights TLS stem modeling as an appropriate method of non-destructive allometric equation development that can immediately impact the magnitude and reduce uncertainty in landscape-level biomass estimates. ${ }^{1}$

\footnotetext{
${ }^{1}$ This chapter is based on: Stovall, A.E.L., Texiera-Anderson, Kristina, Shugart, H.H., In Prep. Assessing terrestrial laser scanning for developing non-destructive biomass allometry. intended for Forest Ecology and Management.
} 


\subsection{Introduction}

Forests contain the vast majority of aboveground biomass and carbon (Houghton et al., 2009), but remain incredibly difficult to measure with a high degree of certainty (Mitchard et al., 2014). Currently, most estimates of forest biomass are inferred rather than measured (Saatchi et al., 2011; Baccini et al., 2012). The most commonly measured variable in studies of forest structure is diameter at breast height $(\mathrm{DBH})$, as this is indicative of several other biophysical variables of interest to forest ecologists and managers - namely biomass. DBH is converted to standing aboveground biomass with empirically derived scaling relationships, or allometric equations. Allometric relationships are created with extensive destructive sampling of trees (Picard et al., 2012) and are essential, as they form the backbone of all landscape-level biomass and carbon mapping (Jenkins et al., 2001). Any error or uncertainty within the relationships ultimately propagates to higher-level estimates (Chen et al., 2015), thus it is imperative they be representative of the trees being measured. For this reason, substantial efforts have been made to create speciesand regionally-specific allometric equations (TerMikaelian \& Korzukhin, 1997) for reducing uncertainty in biomass mapping.

Allometric equations require a high sample size to be dependable, but logistical limitations often limit the number of samples collected in the field (Chave et al., 2004). These limitations primarily stem from the significant cost and labor expense involved with destructive sampling. The destructive sampling process is fraught with difficulties that can lead to measurement errors, ultimately propagating to the final relationships and subsequently to stand-level estimates of biomass. Biomass estimates are even more uncertain in areas of cultural significance due to sampling being prohibited. All of these factors are worsened in areas of complex topography, undesirable climatic conditions, and political restriction, spatially biasing the creation of allometric relationships. The above mentioned issues are especially true in mature forests, where large trees drive the high biomass density of plot measurements (Brown, 1997). Sampling trees at the fringe of current allometry data will reduce uncertainty in stand-level biomass estimates, but the difficulty and complexity of destructive sampling poses a severe limitation.

Non-destructive sampling overcomes nearly every complicating factor associ- 
ated with destructive sampling and opens up avenues for more complex long term ecological research. Efficient non-destructive sampling offers a solution to the issue of insufficient sample size in allometric equations (Chave et al., 2004). The time consuming portion of destructive methods is two-fold: field work and drying. Non-destructive methods exclude this drying step. Moreover, sampling on private and protected land becomes more feasible when the forest is not at a higher risk of degradation from destructive sampling. A unique benefit also arises since measurements can be made over the entire lifespan of the tree, allowing investigation into fine-scale structural changes over time. Finally, increased sampling efficiency from non-destructive approaches may allow even more specific development of allometric equations that take into account environmental variation across the landscape that impacts the growth structure at the scale of the individual tree.

Terrestrial LiDAR, or terrestrial laser scanning (TLS), has become the most viable current option for low-cost non-destructive allometric equation development with increasing levels of accuracy (Calders et al., 2015). Several approaches have been employed for biomass estimation that generally fall into 5 categories (ordered with increasing levels of accuracy): [1] allometric estimation, [2] point structure estimation, [3] voxel derived-volume, [4] quantitative surface models (QSMs), and [5] outer hull models (OHMs). Similar to traditional plot-level approaches, allometric methods rely on measurements of the diameter of the tree with TLS and an allometric equation is used to convert diameter to biomass (Sheridan, 2011; Yao et al., 2011; Seidel et al., 2013). This approach has been applied in many forest types, but disregards information provided by the scanner on tree structure. Point structure estimation is inherited from analysis techniques of airborne LiDAR point clouds. Similar point cloud structure metrics such as total number of returns, height percentiles, and canopy volume are related to allometrically determined biomass (Kankare et al., 2013). This approach has been used to show temporal change in biomass in conifer forests (Srinivasan et al., 2014). However, it is rare that this method is used in concert with destructive samples, essentially negating the benefits of terrestrial LiDAR because the true tree structure is being used to estimate the allometrically derived biomass. Voxel derived methods, described extensively in Chapter 1 , use the structure of the raw point cloud to estimate volume (Hosoi et al., 2013). Infilling of the point cloud is necessary in 
large trees to avoid underestimation of biomass (Bienert et al., 2014). QSMs are the most commonly employed automated method that has a significant amount of validation data (Raumonen et al., 2013; Hackenberg et al., 2014; Calders et al., 2015). While automation is ideal, approximation of tree structure using cylinders is not always appropriate. OHMs attempt to improve upon the QSM method, by directly modeling the trunk surface. OHMs have the lowest documented RMSE\% of all of the aforementioned methods, but coincident validation data is sparse.

Our primary objective is to determine how locally derived terrestrial LiDAR allometry compares to more commonly used allometric relationships at the standlevel. We estimated biomass using QSMs and created several species-specific allometric equations using diameter and height. These equations were compared to the regional and national equivalents in order to assess the differences in slope and RMSE. The impact of sample size and the inclusion of large trees were evaluated for the equations.

\subsection{Methods}

329 individuals of the most dominant canopy species in the SIGEO forest were modeled with TLS data to estimate volume and biomass. These estimates of biomass were the basis of several species-specific allometric equations that we developed using DBH and height as predictor variables. The equations were directly compared to the coefficients and uncertainty of the national allometry. A sample size analysis was conducted to investigate the influence of sample size on coefficient and biomass estimation.

\subsubsection{Site Location and Dominant Species Selection}

The study site is the 25.6 ha (400 m x 600 m) Smithsonian Institute Global Earth Observatory (SIGEO) temperate Large Forest Dynamics Plot in the Smithsonian Conservation Biology Institute near Front Royal, VA (3853'36.6" N, 788'43.4" $\mathrm{W}$; Figure 3.1). We used a stem map at this site with data on all, approximately 40,000, woody stems greater than $1 \mathrm{~cm}$ diameter at breast height (DBH) (Bourg et al., 2013). Each individual stem contains information on the following: DBH, 


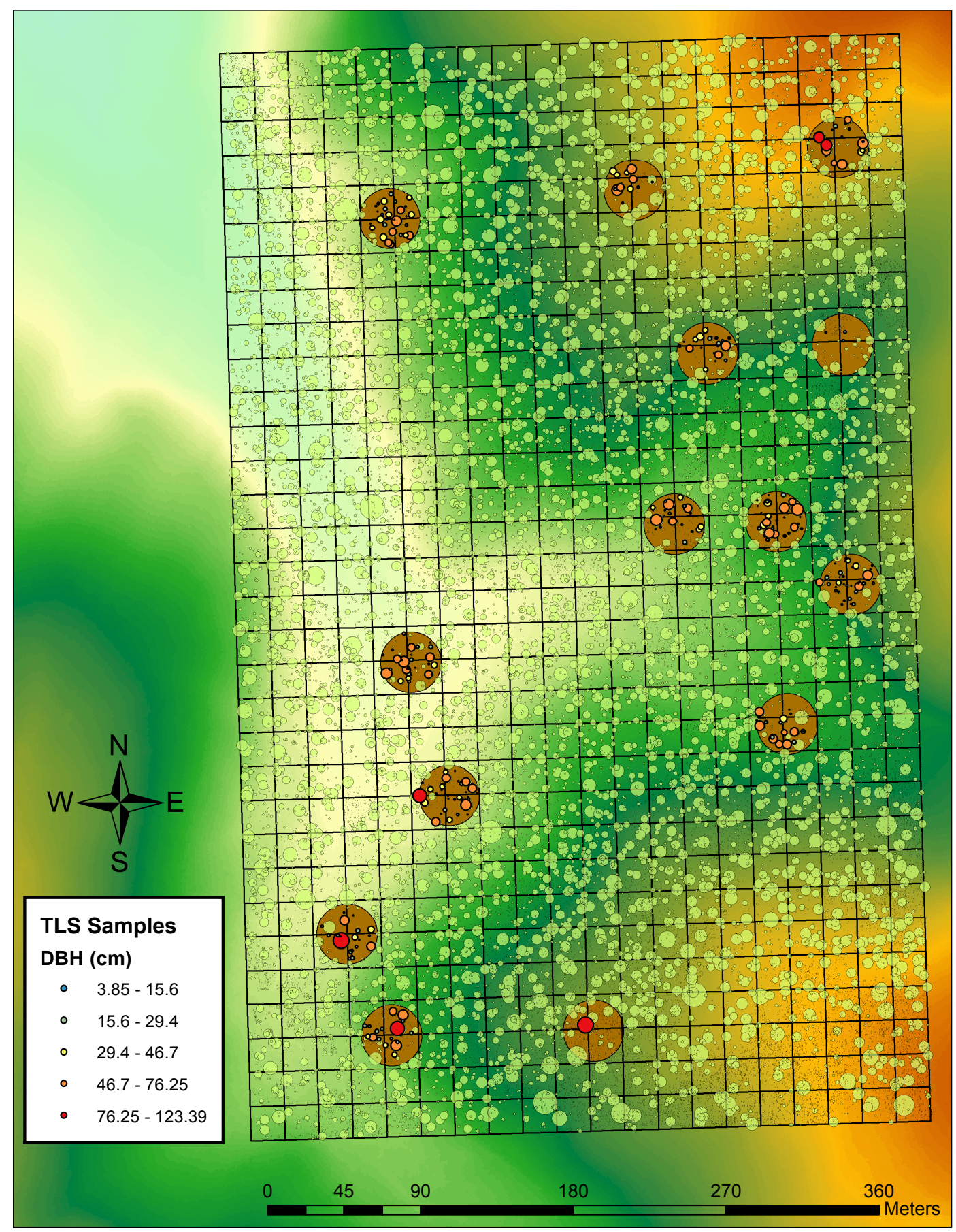

Figure 3.1: Smithsonian Conservation Biology Institute SIGEO forest with stem map (green points) and terrestrial LiDAR plot locations (brown circles). TLS samples are color coded according to diameter and proportionally sized. Elevation is represented in the background (low: cool, high: warm). 
species, x-y coordinates relative to the plot origin. Since this wealth of information is available a priori, determining the most important and influential species for biomass analysis is possible. For each species, an importance value - an index that takes into account the stem density and the dominance of a species - was calculated in order to determine the top 10 most important species across the SIGEO site. We calculated species-specific importance values using:

$$
I V=\frac{\sum_{i=1}^{n} B A_{s p p}+D_{s p p}}{2}
$$

where $B A_{s p p}$ is the basal area and $D_{s p p}$ is the stem density of an individual species in the forest stand. Ultimately, the ten species selected and associated average wood density values $\left(\mathrm{g} \mathrm{cm}^{-3}\right)$ were: Carya cordiformis (0.62), Carya glabra (0.62), Carya ovalis (0.62), Carya tomentosa (0.62), Fagus grandifolia (0.56), Fraxinus americana (0.55), Liriodendron tulipifera (0.40), Quercus alba (0.60), Quercus prinus (0.57), Quercus rubra (0.56) (Chojnacky et al., 2014). These species were used for local allometric equation development, as they were likely to contribute to the majority of biomass within the forest. We estimated biomass using the Chojnacky et al. (2014) equations and determined the top ten species contributed to approximately $80 \%$ of the total forest biomass (Figure 3.2).

\subsubsection{Terrestrial LiDAR Aquisition and Processing Terrestrial LiDAR Sampling}

The 14 1/10th ha circular plot locations were chosen at random using ArcGIS mapping software. Individual plots were located using a handheld Garmin eTrex unit and plot centers were found on the marked grid intersections of the $20 \mathrm{~m}$ SIGEO plot grid network. We reduced occlusion from the presence of high-density vegetation by scanning 5 times in a diamond pattern oriented at approximately each cardinal direction to provide sufficient coverage and a standardized sampling scheme. At times, an additional scan was required for full coverage. White polystyrene registration spheres were placed throughout each plot to aid in digital registration of individual scans, as described in the following section. Additional 

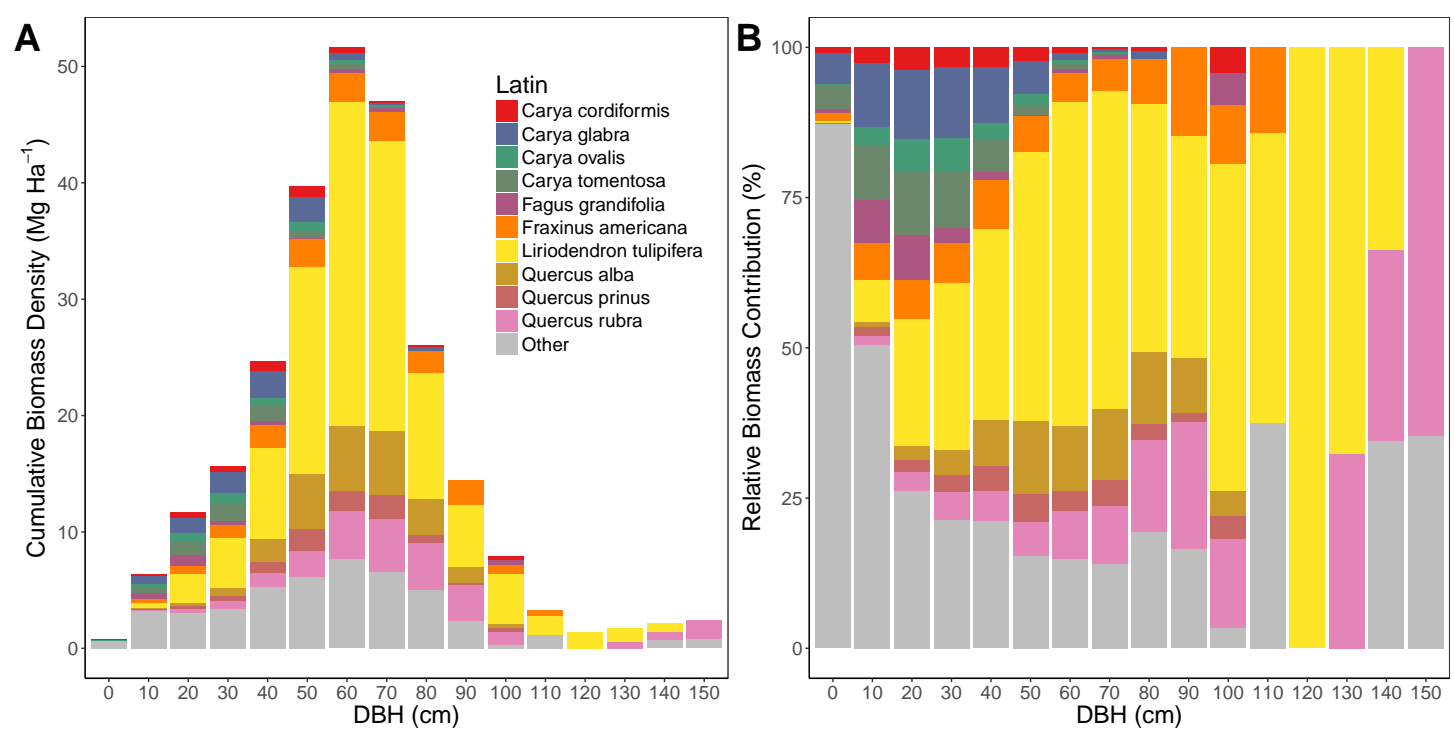

Figure 3.2: The top 10 dominant species contributing over $80 \%$ of biomass within the SIGEO forest as estimated using the Chojnacky et al. (2014) equations. [A] Absolute and $[\mathrm{B}]$ relative contributions to biomass.

tree height measurements were manually collected across a transect following an elevation gradient in the north-direction transect on the site to serve as independent validation of the height allometry created with TLS.

\section{Post Processing}

Individual scans were registered using the automatic registration algorithms included in Faros proprietary software package SCENE and overlapping redundant points were fused together to create a seamless 3D point cloud that could be used for analysis (SCENE 2015). The registration process relied on the spheres used on each field plot. Spheres were located within every scan and aligned with each corresponding sphere within other scans from the same plot. We evaluated the registration error within SCENE. The fully registered scan was then exported with intensity values, column and row numbers, which correspond to scan azimuth and angle, as well as intensity values. 


\section{Volume and Biomass Estimation}

We modeled individual trees using the CompuTree software (Othmani et al., 2011). The processing occured in four steps: (1) ground point classification and DTM creation, (2) stem identification, (3) tree segmentation, and (4) stem reconstruction with quantitative structure models (QSMs). The ground points were identified using a local minima ground estimation algorithm for DTM reconstruction. Stem identification was completed using a nearest neighbor and connected components at DBH height parallel to the DTM. Stem spacing facilitated the identification of unique trees and the point clusters identified using connected components became the initial seed point to initiate cylinder modeling using SimpleTree (Hackenberg et al., 2015). Trees were then automatically segmented from the point cloud with an iterative nearest neighbor approach, moving vertically from the initial seed point along the stem while expanding in area with increasing crown size. The segmented tree was then reconstructed through cylinder fitting with the SimpleTree QSM algorithm. The best fit cylinders were used for creating an allometric relationship between trunk size and branching order, guiding all low certainty cylinder measurements. Gaps in the tree model were filled using this allometric approach, replacing sections with likely cylinder sizes that correspond to branching order. SimpleTree was validated with a number of destructive samples and was found to estimate biomass with approximately 5-15\% error (Hackenberg et al., 2015). Considering our similar forest system in leaf-off conditions, we expected a comparable level of precision and accuracy.

The cylinder models (referred to as TLS models hereafter) provided an estimate of volume, which were converted to estimates of biomass using:

$$
\text { Biomass }=\rho_{s} V_{T L S}
$$

where $V_{T L S}$ is modeled volume $\left(\mathrm{m}^{3}\right)$ and $\rho_{s}$ is average species specific wood density $\left(\mathrm{g} \mathrm{cm}^{-3}\right)$. Biomass estimates were converted to $\mathrm{kg}$ for direct comparison to other allometric equations.. 


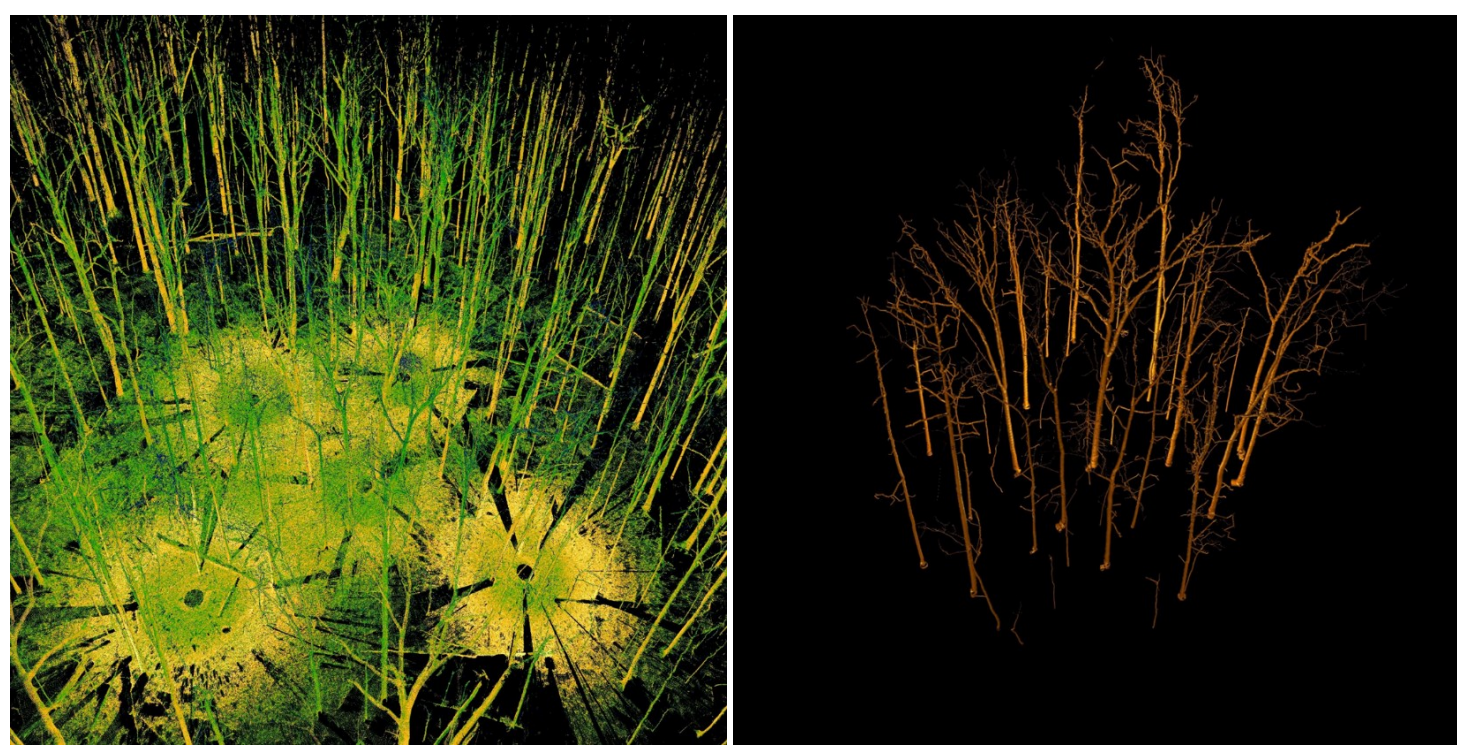

Figure 3.3: Models produced from the SimpleTree algorithm within Computree. Coregistered plot data [left] was used to produce plot-level models of tree structure [right].

\subsubsection{Allometric Development and Analysis}

\section{Allometric Equation Development}

Allometric equations were created for height and biomass estimated using TLS measurments. We used log-linear regression as it is the most common equation form used in national biomass allomery (Jenkins et al., 2003; Chojnacky et al., 2014). Species-specific height allometric relationships were created in the form:

$$
\text { Height }=\exp \left(\beta_{0}+\beta_{1} \ln (D B H)\right)
$$

where $\beta_{0}$ and $\beta_{1}$ are the model coefficients. Log-linear relationships with high RMSE tend to underestimate the predicted value when back-transformed from log-units, so a correction factor has been proposed to remove this bias (Sprugel, 1983). The correction factor uses the mean square error (MSE) and is calculated as:

$$
C F=e^{\frac{M S E}{2}}
$$


The application of this correction factor in small sample sizes has been found to bias equations incorrectly (Flewelling \& Pienaar, 1981), so was only implemented in cases of high equation sample size and high equation RMSE.

Species-specific biomass allometry was developed using three different equation forms with predictors: height, diameter, and diameter and height. Least-squares linear regression was used on the log transformed variables across all biomass equations to determine the scaling coefficient, $\beta_{1}$, and intercept, $\beta_{0}$ in the form:

$$
\begin{gathered}
\text { Biomass }=\exp \left(\beta_{0}+\beta_{1} \ln (\text { Height })\right) \\
\text { Biomass }=\exp \left(\beta_{0}+\beta_{1} \ln (D B H)\right) \\
\text { Biomass }=\exp \left(\beta_{0}+\beta_{1} \ln \left(D B H^{2} \text { Height }\right)\right)
\end{gathered}
$$

\section{Allometric Equation Evaluation}

Each allometric equation was evaluated in terms of the coefficient of determination $\left(\mathrm{R}^{2}\right)$ and uncertainty. Model uncertainty was expressed in terms of RMSE in the form:

$$
R M S E=\sqrt{\frac{\sum_{i=1}^{n}\left(y_{i}-\widehat{y}_{i}\right)^{2}}{n}}
$$

RMSE was calculated in log units for comparison to national equations, but relative RMSE (\%) was reported as the value is more representative of equation uncertainty. Considering RMSE is not constant, but increases with increasing diameter we estimated relative RMSE by calculating the mean relative RMSE at $10 \mathrm{~cm}$ intervals of tree diameter. Relative RMSE at each diameter interval was calculated as the RMSE divided by the mean biomass of that interval and tended to remain approximately constant across this range. The equation-level relative RMSE was reported as the average of relative RMSE estimates across all diameter increments. Our estimate of relative RMSE is more representative of equation error compared to the RMSE in log units typically reported because it can be directly applied to a biomass estimate and produce an estimate of uncertainty in units of biomass $(\mathrm{kg})$. 


\section{Sample Size Analysis}

The effect of equation sample size on the two $\beta$ coefficients was evaluated through iterative random sampling ranging from 5 to 329 samples. A random sample was selected at each sample size and a model fit in each of the forms described above. This process was repeated 1000 times at each successive sample size and the mean coefficient across all iterations determined. The effect of large trees on the model coefficients with respect to sample size was evaluated using the same process, but excluding those trees above $50 \mathrm{~cm} \mathrm{DBH}$. The coefficients of the final full sample size model were considered the benchmark of comparison. We used the coefficients to estimate the biomass of a tree with a $50 \mathrm{~cm}$ diameter and $35 \mathrm{~m}$ in height. These diameter and height values were based on our a priori analysis of site-level biomass estimates and are approximately representative of the average tree size contributing the most biomass to the forest (Figure 3.2). We compared three scenarios to evaluate equation error: 1) sample size-dependent error in the full model, sample size dependent error in the model excluding trees above $50 \mathrm{~cm}$, and error of the model excluding trees above $50 \mathrm{~cm}$ from the full model.

\section{Comparison to National Equations}

TLS allometry was directly compared to the most commonly applied national equations in the US (Jenkins et al., 2003; Chojnacky et al., 2014). A tree level comparison was used to evaluate national and TLS-based allometric equations to the TLS models. Absolute and relative RMSE and bias were used to evaluate all equations. Bias was calculated as:

$$
\text { Bias }=\frac{\sum_{i=1}^{n}\left(y_{i}-\widehat{y}_{i}\right)}{n}
$$

Species-specific equation RMSE in log-units was directly compared to the national equations. We were unable to include the Chojnacky et al. (2014) equations in this comparison because RMSE values were not reported. 
Table 3.1: Summary statistics for all TLS modeled trees and predicted biomass.

\begin{tabular}{|c|c|c|c|c|c|}
\hline Statistic & $\mathrm{N}$ & Mean & St. Dev. & Min & Max \\
\hline Field measured DBH $(\mathrm{cm})$ & 258 & 28.600 & 22.024 & 3.850 & 123.390 \\
\hline TLS measured DBH $(\mathrm{cm})$ & & 27.995 & 21.024 & 3.850 & 120.090 \\
\hline Height $(m)$ & & 20.700 & 8.550 & 7.080 & 36.7 \\
\hline Volume $\left(m^{3}\right)$ & & 1.940 & 3.110 & 0.013 & 25.200 \\
\hline Specific Gravity $\left(\mathrm{g} / \mathrm{cm}^{3}\right)$ & & 0.566 & 0.092 & 0.400 & 0.729 \\
\hline TLS 3D Model Biomass $(\mathrm{kg})$ & & 997 & 1,517 & 8 & 11,179 \\
\hline TLS Height Allometry Biomass $(\mathrm{kg})$ & & 1,047 & 1,110 & 10 & 4,227 \\
\hline TLS Diameter Biomass $(k g)$ & & 1,000 & 1,616 & 4 & 12,672 \\
\hline TLS Diameter-Height Biomass $(\mathrm{kg})$ & & 974 & 1,438 & 5 & 10,332 \\
\hline Chojnacky et al. (2014) ( $k g)$ & & 753 & 1,348 & 3 & 10,747 \\
\hline Jenkins et al. (2003) $(\mathrm{kg})$ & & 802 & 1,485 & 3 & 12,229 \\
\hline Jenkins et al. (2003) with CF $(\mathrm{kg})$ & & 928 & 1,729 & 3 & 14,645 \\
\hline
\end{tabular}

\subsection{Results}

\subsubsection{Allometry}

\section{Height Allometry}

The generalized TLS based height allometry was completed on 258 individual trees with height ranging from 7 to $36.7 \mathrm{~m}$, while tree diameter spanned from 4 to 124 $\mathrm{cm}$. Height was directly derived from the TLS models, which were most reliable in larger trees. For this reason, the minimum height was greater than would be expected in a study relying entirely on manual measurements. Height allometry had a high RMSE, leading us to apply a correction factor for the predictions from this equation. The generalized equation had a high sample size and, while exhibiting high variability, accurately predicted tree height $(\mathrm{RMSE}=18.9 \%)$ in our manual validation with little bias $(6 \%)$ post correction. Species specific height equations performed better than the general equation in the validation both in terms of of RMSE (13\%) and bias (-0.8\%). 
Table 3.2: General biomass allometry for 271 modeled trees.

\begin{tabular}{lcccccc}
\hline \hline & $\beta_{0}$ & $\beta_{0}[s e]$ & $\beta_{1}$ & $\beta_{1}[s e]$ & $R^{2}$ & RMSE (\%) \\
\hline $\mathrm{H}$ & -5.040 & 0.214 & 3.680 & 0.072 & 0.907 & 68.6 \\
$\mathrm{DBH}$ & -1.740 & 0.090 & 2.370 & 0.028 & 0.965 & 29.9 \\
$\mathrm{DBH}^{2} \mathrm{H}$ & -2.750 & 0.087 & 0.919 & 0.009 & 0.974 & 22.3 \\
$\mathrm{DBH}^{2} \mathrm{H} \rho$ & -2.470 & 0.073 & 0.948 & 0.008 & 0.980 & 20.4 \\
\hline
\end{tabular}

\section{Biomass Allometry}

A total of 30 different species specific allometric equations were created in three different forms for 10 species from 258 individual trees using diameter and height as predictive variables. An additional 4 site-wide general allometric equation were created with 271 modeled individuals (Table 3.2).

The species-specific equations ranged in sample size from 10 to 47 individuals (Table 3.3). Liriodendron tulipifera had the greatest range of diameter values (up to $120 \mathrm{~cm}$ ) for a single equation, followed by Carya glabra. The inclusion of height reduced RMSE in all species-specific allometric equations. Carya cordiformis had the highest RMSE of all species in the height-based equation but combining diameter with height improved this significantly (reduced from 53.5\% to $35.9 \%$ ). Fraxinus americana, Liriodendron tulipifera, and Quercus rubra all had the lowest RMSE of any diameter (15.8\%,12.2\%, and 12\% respectively) and diameter-height $(10.8 \%, 12 \%$, and $13.7 \%$ respectively) allometry. The Liriodendron tulipifera height-diameter equation retained low RMSE while having the highest sample size.

Four generalized allometric equations were created with 271 TLS models using diameter, height, and wood density (Table 3.2). Height allometry was the most uncertain (see Figure 3.8b), losing predictive power beyond approximately $30 \mathrm{~m}$ and $3000 \mathrm{~kg}$. Including height and wood density improved diameter allometry RMSE from $30 \%$ to $20 \%$. 

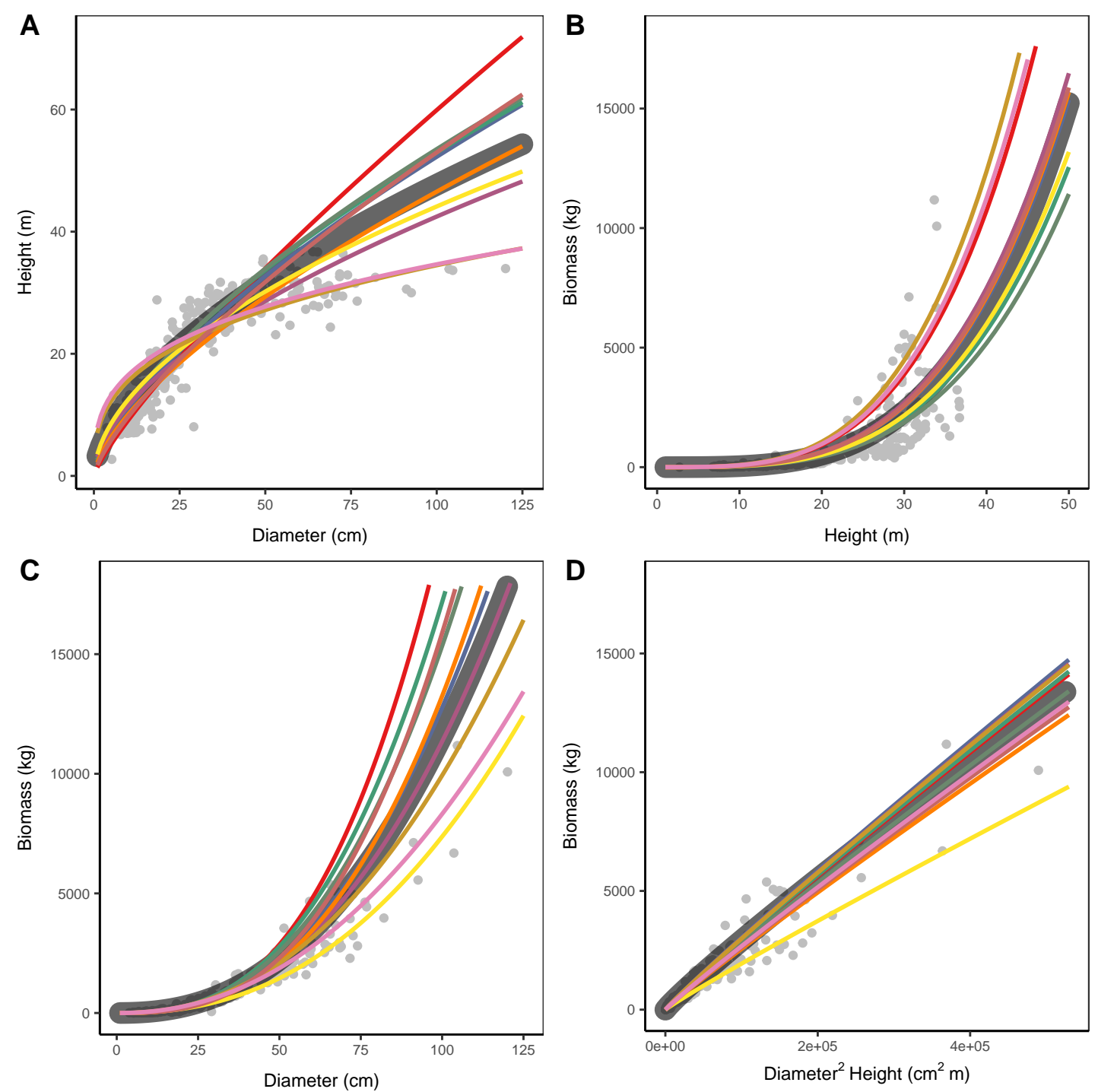

Figure 3.4: Species specific allometry for $[\mathrm{A}]$ tree height predicted from diameter and biomass allometry derived from TLS models with $[\mathrm{A}]$ diameter, $[\mathrm{B}]$ untransformed diameter-height, and $[\mathrm{C}] \log$ transformed diameter-height predictive models. Generalized allometry for each equation form is shown in dark grey. 
Table 3.3: Species-Specific allometry for the 10 most dominant species at the study site using height and diameter as predictors. Blank spaces indicate the same coefficient (top row in each section) was used across all species.

\begin{tabular}{|c|c|c|c|c|c|c|c|c|c|}
\hline & Species & $\mathrm{n}$ & range & $\beta_{0}$ & $\beta_{0}[\mathrm{se}]$ & $\beta_{1}$ & $\beta_{1}[\mathrm{se}]$ & $R^{2}$ & RMSE (\%) \\
\hline $\mathbf{H}$ & $\begin{array}{l}\text { Carya cordiformis } \\
\text { Carya glabra } \\
\text { Carya ovalis } \\
\text { Carya tomentosa } \\
\text { Fagus grandifolia } \\
\text { Fraxinus americana } \\
\text { Liriodendron tulipifera } \\
\text { Quercus alba } \\
\text { Quercus prinus } \\
\text { Quercus rubra }\end{array}$ & $\begin{array}{l}10 \\
41 \\
13 \\
22 \\
14 \\
14 \\
47 \\
25 \\
11 \\
17\end{array}$ & $\begin{array}{l}7-30 \mathrm{~m} \\
8-32 \mathrm{~m} \\
12-28 \mathrm{~m} \\
9-29 \mathrm{~m} \\
10-34 \mathrm{~m} \\
10-32 \mathrm{~m} \\
10-37 \mathrm{~m} \\
15-32 \mathrm{~m} \\
8-29 \mathrm{~m} \\
17-32 \mathrm{~m}\end{array}$ & $\begin{array}{l}-3.8569 \\
-4.0344 \\
-4.1608 \\
-4.3059 \\
-3.9979 \\
-3.9606 \\
-4.2275 \\
-3.4588 \\
-3.9593 \\
-3.726\end{array}$ & $\begin{array}{l}0.3573 \\
0.1833 \\
0.2198 \\
0.1975 \\
0.2151 \\
0.2144 \\
0.1889 \\
0.2013 \\
0.2293 \\
0.2132\end{array}$ & 3.3427 & 0.1134 & $\begin{array}{c}0.74 \\
0.78 \\
0.81 \\
0.84 \\
0.8 \\
0.94 \\
0.83 \\
0.68 \\
0.89 \\
0.7\end{array}$ & $\begin{array}{l}53.52 \\
28.46 \\
39.01 \\
49.89 \\
36.37 \\
31.38 \\
26.22 \\
32.38 \\
22.59 \\
30.92\end{array}$ \\
\hline DBH & $\begin{array}{l}\text { Carya cordiformis } \\
\text { Carya glabra } \\
\text { Carya ovalis } \\
\text { Carya tomentosa } \\
\text { Fagus grandifolia } \\
\text { Fraxinus americana } \\
\text { Liriodendron tulipifera } \\
\text { Quercus alba } \\
\text { Quercus prinus } \\
\text { Quercus rubra }\end{array}$ & $\begin{array}{l}10 \\
41 \\
13 \\
22 \\
14 \\
14 \\
47 \\
25 \\
11 \\
17\end{array}$ & $\begin{array}{l}12-40 \mathrm{~cm} \\
11-47 \mathrm{~cm} \\
11-46 \mathrm{~cm} \\
10-41 \mathrm{~cm} \\
13-105 \mathrm{~cm} \\
11-55 \mathrm{~cm} \\
13-120 \mathrm{~cm} \\
21-76 \mathrm{~cm} \\
11-50 \mathrm{~cm} \\
13-93 \mathrm{~cm}\end{array}$ & $\begin{array}{l}-2.8734 \\
-1.7925 \\
-1.4224 \\
-2.8017 \\
-2.1853 \\
-2.7828 \\
-1.9136 \\
-1.1891 \\
-3.1592 \\
-0.8702\end{array}$ & $\begin{array}{c}0.5908 \\
0.6769 \\
0.8474 \\
0.7125 \\
0.7428 \\
0.6962 \\
0.648 \\
0.8096 \\
0.868 \\
0.7284\end{array}$ & $\begin{array}{l}2.7603 \\
2.4404 \\
2.3466 \\
2.7712 \\
2.5399 \\
2.6764 \\
2.3513 \\
2.2568 \\
2.7866 \\
2.1491\end{array}$ & $\begin{array}{l}0.1927 \\
0.2198 \\
0.2697 \\
0.2364 \\
0.2381 \\
0.2259 \\
0.2055 \\
0.2399 \\
0.2651 \\
0.2229\end{array}$ & $\begin{array}{l}0.86 \\
0.93 \\
0.94 \\
0.93 \\
0.95 \\
0.99 \\
0.98 \\
0.91 \\
0.98 \\
0.98\end{array}$ & $\begin{array}{c}52.11 \\
22.61 \\
21.1 \\
40.07 \\
21.59 \\
15.8 \\
12.17 \\
25.76 \\
21.22 \\
11.95\end{array}$ \\
\hline $\mathrm{DBH}^{2} \mathbf{H}$ & $\begin{array}{l}\text { Carya cordiformis } \\
\text { Carya glabra } \\
\text { Carya ovalis } \\
\text { Carya tomentosa } \\
\text { Fagus grandifolia } \\
\text { Fraxinus americana } \\
\text { Liriodendron tulipifera } \\
\text { Quercus alba } \\
\text { Quercus prinus } \\
\text { Quercus rubra }\end{array}$ & $\begin{array}{l}10 \\
41 \\
13 \\
22 \\
14 \\
14 \\
47 \\
25 \\
11 \\
17\end{array}$ & $\begin{array}{l}12-40 \mathrm{~cm} ; 7-30 \mathrm{~m} \\
11-47 \mathrm{~cm} ; 8-32 \mathrm{~m} \\
11-46 \mathrm{~cm} ; 12-28 \mathrm{~m} \\
10-41 \mathrm{~cm} ; 9-29 \mathrm{~m} \\
13-105 \mathrm{~cm} ; 10-34 \mathrm{~m} \\
11-55 \mathrm{~cm} ; 10-32 \mathrm{~m} \\
13-120 \mathrm{~cm} ; 10-37 \mathrm{~m} \\
21-76 \mathrm{~cm} ; 15-32 \mathrm{~m} \\
11-50 \mathrm{~cm} ; 8-29 \mathrm{~m} \\
13-93 \mathrm{~cm} ; 17-32 \mathrm{~m}\end{array}$ & $\begin{array}{l}-2.9176 \\
-2.9001 \\
-2.8996 \\
-2.9834 \\
-2.9557 \\
-3.0639 \\
-3.3683 \\
-2.9251 \\
-3.0527 \\
-3.0373\end{array}$ & $\begin{array}{c}0.132 \\
0.0781 \\
0.0934 \\
0.0844 \\
0.0918 \\
0.0916 \\
0.0804 \\
0.087 \\
0.0978 \\
0.0915\end{array}$ & 0.9494 & 0.0126 & $\begin{array}{l}0.91 \\
0.94 \\
0.94 \\
0.96 \\
0.97 \\
0.99 \\
0.99 \\
0.93 \\
0.98 \\
0.98\end{array}$ & $\begin{array}{c}35.87 \\
17.68 \\
21.6 \\
27.89 \\
19.04 \\
10.78 \\
11.96 \\
23.42 \\
21.12 \\
13.69\end{array}$ \\
\hline
\end{tabular}

\subsubsection{Sample Size Analysis}

The sample size analysis had several consistent trends across all different equation types: (1) sharp deviation followed by a leveling off near the final coefficient with increasing sample size, (2) a clear difference in equation coefficient when large trees are removed from the training set, and (3) higher variability in the coefficients with a lower diameter range. Low sample size equation coefficients deviate from the benchmark coefficient in all equation forms. In the diameter-based equation this difference was greatest below 180 samples. The diameter-height equation stabilized near 100 samples, but had the lowest variability of any of the equations, regardless of sample size. Equations excluding trees above $50 \mathrm{~cm}$ tended to over- or underestimate the model coefficient and did not approach the benchmark coefficient created using the full set of tree models (Figure 3.6). The diameter- 

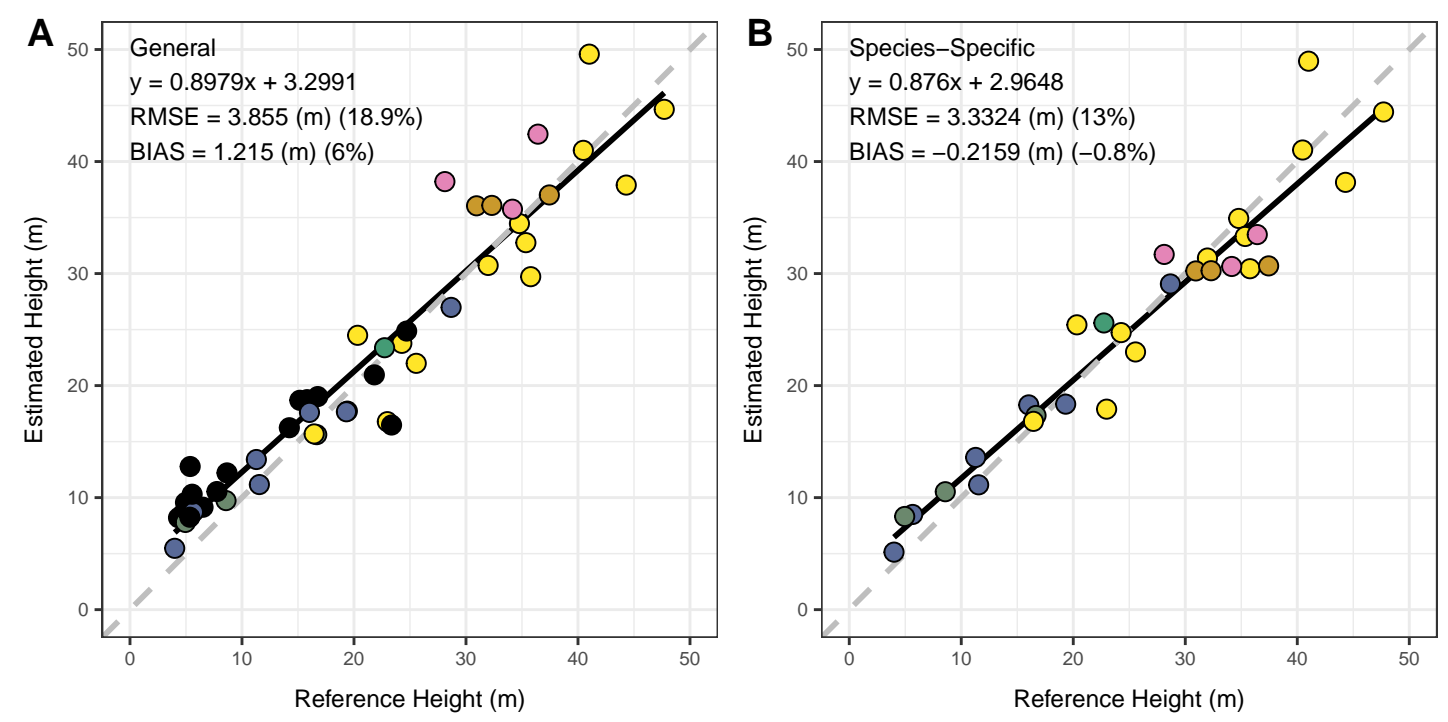

Figure 3.5: Height model validation with $[\mathrm{A}]$ general and $[\mathrm{B}]$ species specific equations. All trees used for validation were used to evaluate the generalized height allometry, while the species specific equation only compared species captured in the allometry. Species not represented in the general allometric model are black in color. Height estimates were made with a correction factor applied, as this equation had high sample size and high RMSE.

height coefficient reached the benchmark coefficient at a lower sample size than the equation created without trees above $50 \mathrm{~cm}$ (100 samples versus 200 samples). Higher sample size stability is apparent in both coefficients. Error in biomass estimates were highest in the height equation excluding large trees (approximately $30 \%$ ), leading to an overestimation. In contrast, the diameter equation excluding large trees underestimated biomass. The diameter-height equation had the most resilience to large tree removal.

\subsubsection{Comparison to National Equations}

The compiled biomass predictions using the national scale equations underestimated biomass on average in trees above $2500 \mathrm{~kg}$. Chojnacky et al. (2014) and Jenkins et al. (2003) underestimated tree-level biomass (-24.5\% and $-19.5 \%$ bias, respectively) and had comparable uncertainty (41.1\% and $44.7 \%$, respectively). A correction factor removed much of the bias in the Jenkins equations (-7\%), but led 

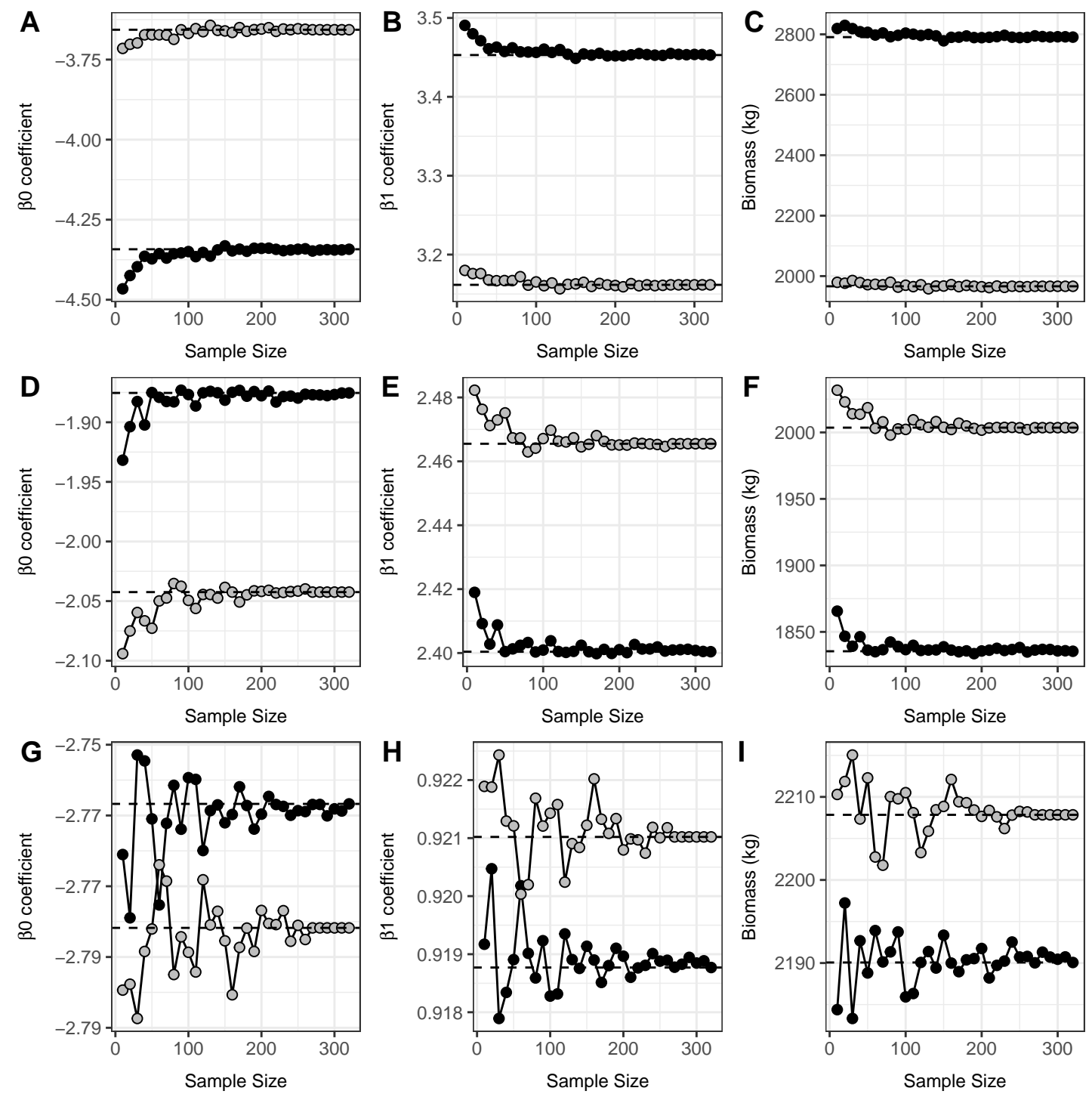

Figure 3.6: Sensitivity of generalized height [top], diameter [middle], and diameterheight [bottom] models of biomass allometry. [left] $\beta_{0}$ and [middle] $\beta_{1}$ coefficients stabilize at approximately 180 samples for the diameter based model and 100 for the diameter and height model. The equation coefficients for the model excluding large trees (black) never reaches the benchmark coefficients (dashed line) of models including large trees. 

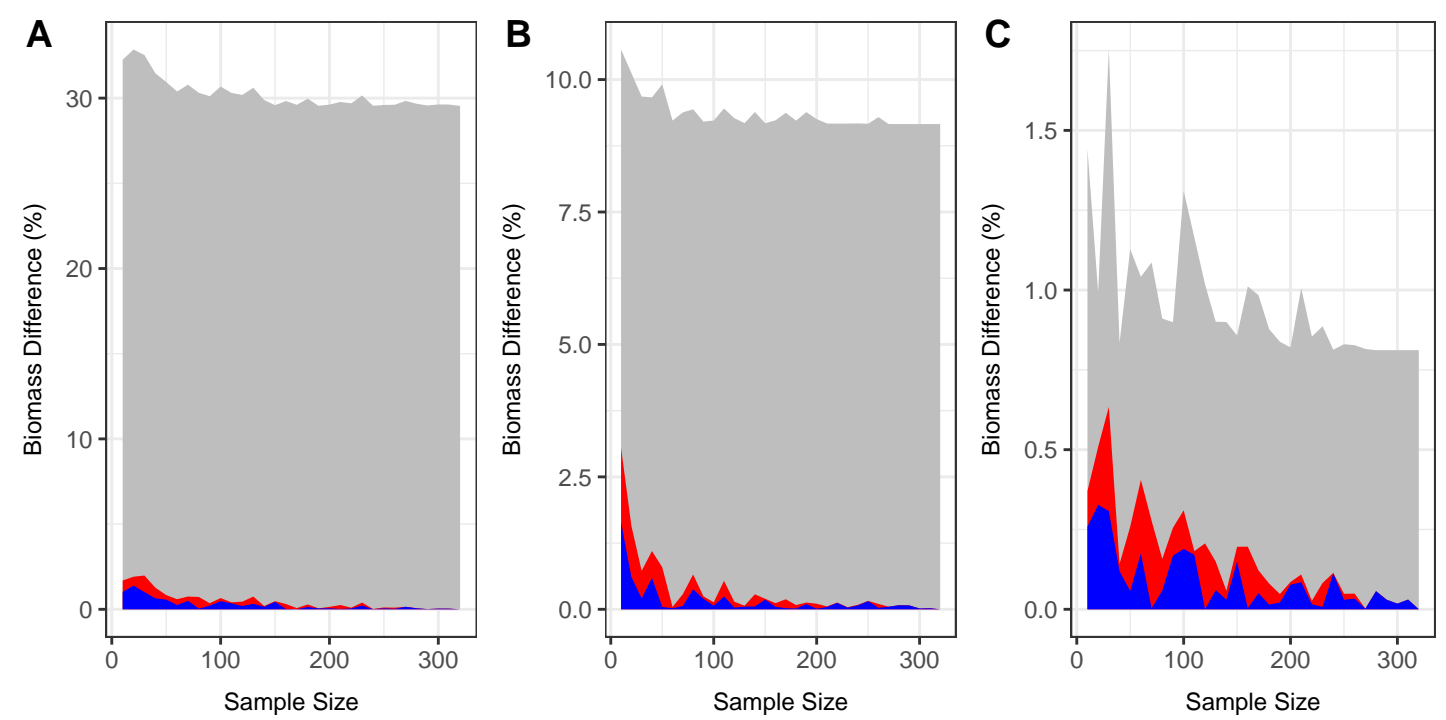

Figure 3.7: Comparison of percent error in biomass from the full allometric models using $[\mathrm{A}]$ height, $[\mathrm{B}]$ diameter, and height-diameter as predictors. Three scenarios were compared: 1) sample size-dependent error in the full model (blue), 2) sample size dependent error in the model excluding trees above $50 \mathrm{~cm}$ (red), and error of the model excluding trees above $50 \mathrm{~cm}$ from the full model (grey). The height equation excluding large trees deviated approximately 30\%, while diameter (10\%) and height-diameter (less than 1\%) were less affected by exclusion of large trees. The inclusion of large trees leads to less model error compared to increased sample size.

to increased uncertainty (55.3\%). TLS height allometry performed well in small trees, but was unable to accurately predict large tree biomass. TLS diameter and diameter-height allometry had lower uncertainty than the national equations (34.3\% and $27.6 \%$, respectively) and were nearly unbiased. Certain species agree more with the national equations (Liriodendron tulipifera, Fraxinus americana, and Fagus grandifolia) while others display a consistent underestimate of biomass. The mean RMSE of the TLS allometry is comparable to the error reported in the Jenkins equations (Figure 3.9), but the inclusion of height in the TLS allometry reduced RMSE below this value. The range of RMSE was also reduced upon including height in the TLS allometry. 
A

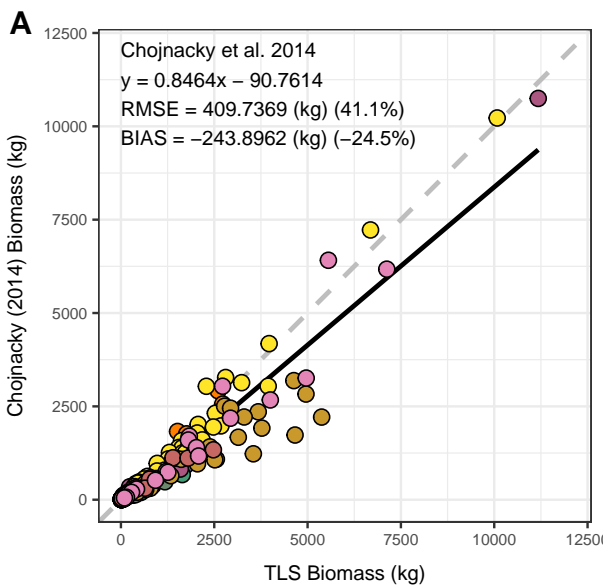

C
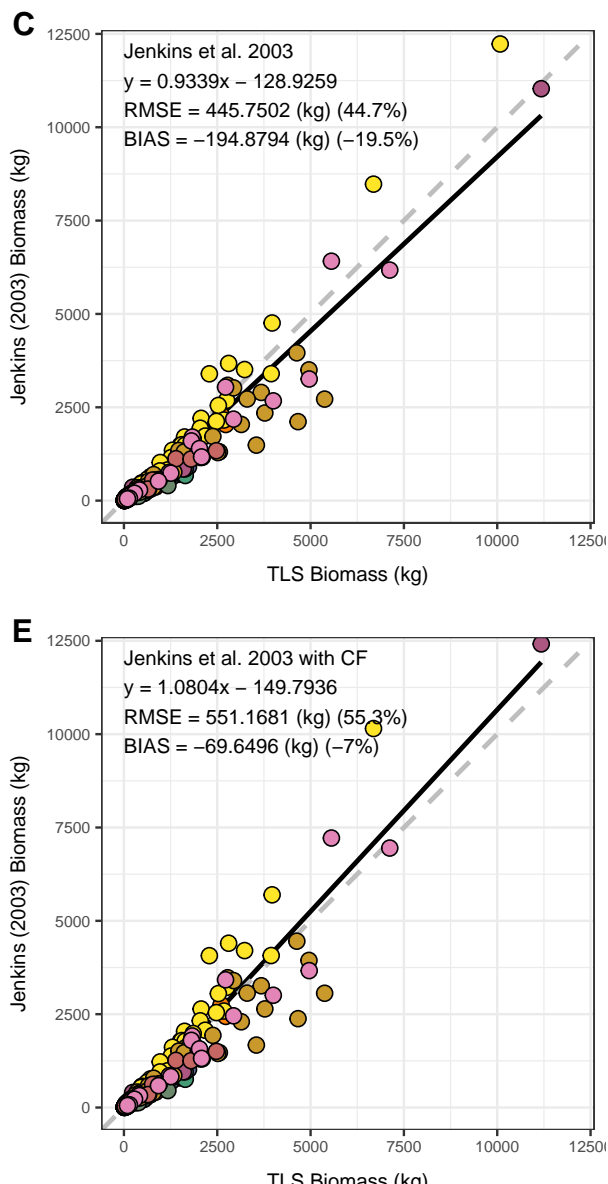
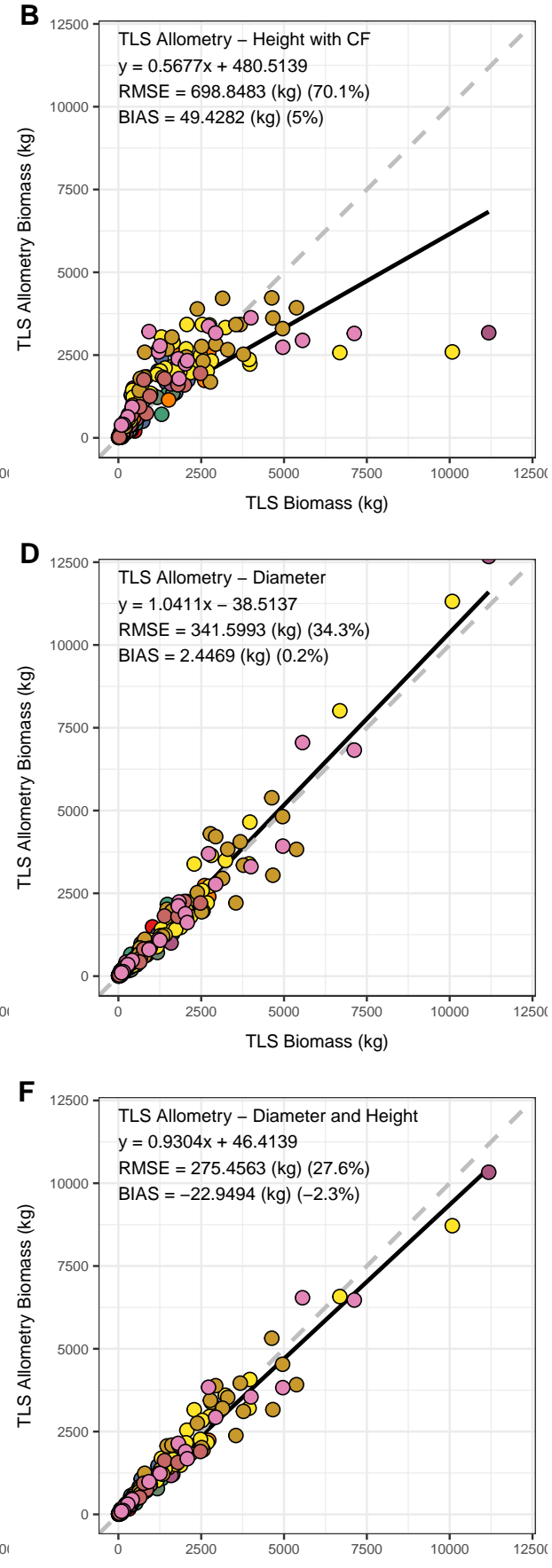

Figure 3.8: Comparison of $[\mathrm{Ag}]$ Chojnacky et al. 2014, [L [B] TLS height allometry [C] Jenkins et al. (2003), [D] TLS diameter allometry [E] corrected Jenkins et al. (2003), and [F] TLS diameter-height allometry biomass to TLS derived biomass for all tree models. 


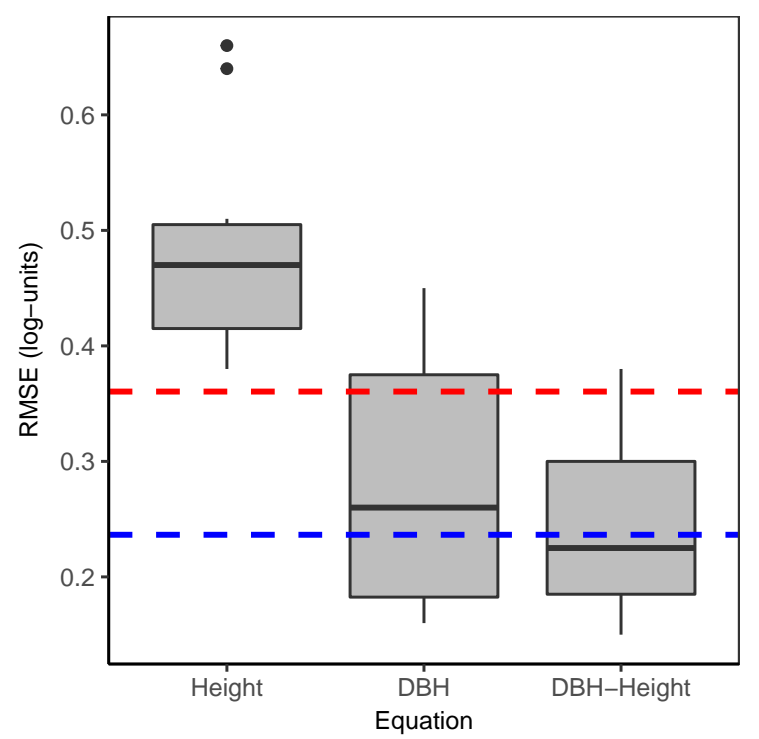

Figure 3.9: Box-plot comparing range of observed RMSE (log units) for three TLS equation forms to Jenkins et al. 2003 RMSE. The Hard maple / oak / hickory / beech equation (blue) and Mixed Hardwood (red) equation RMSEs are indicated with the dotted horizontal lines. *Note: Chojnacky et al. 2014 equation RMSE was not reported so values could not be compared.

\subsection{Discussion}

Terrestrial LiDAR is an effective tool for non-destructively estimating in situ individual tree biomass for developing allometry. The work presented highlights the efficiency and utility of implementing a standardized approach for large area TLS acquisition with the goal of creating high sample size allometric equations. Improvements in allometry are the fastest way to improve biomass mapping. As such, we present a framework for moving from acquisition to allometry that can be replicated in other deciduous forests. Moreover, we show that local allometric equations are not representative if large trees are not included and/or if sample size is insufficient. 


\subsubsection{Allometry}

\section{Height Allometry}

The independent validation showed species specific and generalized height models accurately estimated tree height. We found a consistent underestimation of height in the tallest trees in the validation data set, but attributed this almost entirely to the bias from log transformed regression, as our equations performed well post bias-correction. Given the presence of underestimation after the application of a correction factor, several other possibilities may have caused this underestimation. TLS has been reported to underestimate tree height in dense vegetation (Hopkinson et al., 2004). As such, the trend in underestimating height may be driven by upper canopy occlusion (Srinivasan et al., 2014). Occlusion can potentially be solved through improvements in sampling design (Wilkes et al., 2017) or through sampling with multiple return instruments. The main benefit garnered from using TLS for height estimates of trees is the potential to acquire less biased estimates than manual measurements. However, manually measured height has the potential to be unreliable in dense forest ecosystems (Calders et al., 2015), but, given leaf-off conditions, we expect our validation to be accurate and unbiased.

\section{Biomass Allometry}

In order to disentangle the effect of wood density on general trends in biomass we created species-specific biomass allometry. Grouping equations at the species level controls for average species wood density and the relationship is representative of the sheer wood volume, reflected in Table 3.5 (Appendix). The most apparent finding from the comparison of height-diameter biomass allometry was the convergence of all equations to a very similar slope coefficient. As trees tend to optimize growth strategies, this trend aligns closely with the expected physiological processes driving tree growth - filling three dimensional space in the canopy in order to maximize photosynthetic input and reduce competition. Moreover, since our predictive variables are a combination of diameter and height the resulting value closely resembles volume. As such, we would expect a close linear relationship, where any variation in equation coefficient is representative of changes in wood al- 
location with respect to an equivalent diameter and height. Even still, the inclusion of height did not completely remove species specific variation in wood allocation (Appendix, Table 3.5). Fagus grandifolia had the greatest amount of wood volume for a given diameter and height, but the equation was likely heavily influenced by a single high diameter and low height (102 cm and $33.7 \mathrm{~m}$, respectively) individual. The next most voluminous species was the canopy dominant Quercus alba, suggesting these species efficiently use three dimensional canopy space. In contrast, Carya tomentosa had less total wood allocation for a given diameter and height, a trend consistent with the species structure over the sampled diameter range, allocating the majority of the wood to the central leader. These types of trends are effectively captured since wood density is not a confounding factor in the estimation of wood volume as captured with TLS.

The separation of wood density from biomass has implications for improved national-scale allometric equation development. Considering trees are adaptable organisms responding to their growth environment a volume-based approach to equation development may provide greater flexibility of biomass estimation on larger spatial scales. Evidence for allometric equation coefficient variation across large spatial scales underscores the need for region-specific allometry (Duncanson et al., 2015b), but wood density variation must also be accounted for (Chave et al., 2006). The creation of species specific volume equations that capture structural variation and spatial distributions of average species-specific density would capture the primary factors contributing to biomass uncertainty in forest measurement. With TLS this can potentially be completed with high sample size regionand species-specific volume allometry with associated density estimates that are representative of the growth environment.

The clear benefit of using TLS for allometric development is emphasized in the biomass estimates above $50 \mathrm{~cm}$. The largest tree sampled (Liriodendron tulipifera) was well beyond the size of a typical destructively sampled tree with a diameter of $123 \mathrm{~cm}$ and weighing approximately 11,1779 kg. Equation coefficients are constrained when large trees are present, as is the case with the Quercus, Fagus grandifolia, and Liriodendron tulipifera equations. The coefficients observed for equations lacking large trees are potentially unrealistic when expanded to the larger diameter size. For example, though the species mentioned had large indi- 
viduals included in the final equations, the coefficients remained lower than other equations without large trees. This trend suggests, allometric equations with insufficient samples in large diameter classes will lead to an overestimate of biomass when applied to more massive trees, as discussed in the next section. Considering large trees can hold approximately $40 \%$ of stand-level biomass (Brown, 1997), increasing sample size for the larger diameter class in allometry could potentially drastically alter our understanding of forest-wide biomass allocation.

\subsubsection{Sample Size Analysis}

Sample size strongly impacted both equation coefficients for all equation forms. The inclusion of height reduced the number of samples required to create a strong and stable allometric equation. The diameter only equation did not stabilize until nearly 200 samples, but when including height, the point of stabilization reduced to around 100 samples (Figure 3.6). Overall, the sample sizes required for stable allometry are well beyond the typical average equation sample sizes of less than 100 . This finding is in line with others showing a consistent positive bias using a similar approach with millions of samples collected using airborne LiDAR (Duncanson et al., 2015a). We reached a similar conclusion at a lower sample size and at a smaller spatial scale, but, nonetheless, emphasize the importance of increasing sample size in allometric equations for biomass.

Inclusion of large trees in the sample size analysis of diameter and diameterheight allometry reinforced our findings concerning the tenancy of equations to overestimate biomass when excluding large trees. This trend was apparent when considering all trees, rather than on a species specific basis. At low sample sizes the equations lacking large trees were more prone to overestimate biomass. Furthermore, the rate at which the equation stabilized when large trees were excluded was substantially lower. Figure 3.6 D-I are a clear example of this phenomenon - without trees above $50 \mathrm{~cm}$ about 100 more samples are needed to reach a stable equation. Moreover, equations excluding large trees never actually reach the expected equation coefficients. In contrast, the loss of large trees in the height biomass equations led to a substantial underestimate of biomass, suggesting remote-sensing derived biomass from tree height measurements may be impacted substantially if 
equations neglect large trees. Large trees must be included in allometry in order for for them to be representative across the entire range of diameter and height. Our analysis underscores the importance of using TLS as a means of capturing these trends in the largest trees in the forest to more accurately estimate forest biomass.

Considering TLS provides an efficient non-destructive means to estimate biomass of trees, the logistical considerations of creating or using each equation type should be considered. A diameter-only equation potentially holds the greatest utility, as it can easily be applied to many readily available forestry measurements. Height measurements may not be available on all forest inventory plots and can be unreliable due to difficulty of field measurements. However, as this analysis has shown, the inclusion of height reduces equation variability and the number of samples required for a stable allometric equation. Moreover, if uncertainty in plot-level biomass is the ultimate goal, height should be included in allometry, as the reduction in residual equation error is substantial. TLS biomass acquisition reduces the traditional limitations of high sample size allometry, as it can measure biomass accurately and non-destructively. Our recommendation is for the future adoption of this TLS framework to create a range of equations using easily measurable parameters - from diameter and height in field campaigns to crown diameter using remote sensing (Jucker et al., 2017). A multifaceted approach allows the greatest potential benefits for reducing uncertainty as it will offer improvements regardless of the level of field data available.

\subsubsection{National Equation Comparison and Evaluation}

The Chojnacky et al., (2014) and Jenkins et al. (2003) equations are less representative at higher values of biomass. This observation underscores the primary limitation of applying these equations at the scale of the individual tree. Most of the national allometric equations were built with equations from multiple locations and are not meant to be representative at a small scale. Moreover, very few large diameter trees are included in these equations, so this is the range where they should be least representative. However, the sample of trees included in this analysis is not sufficient to strongly reinforce this conclusion. Though, the gen- 
eral trend in disagreement increases with increasing biomass, several high biomass trees have good agreement with the national scale allometry. Excluding these high biomass trees causes a stronger negative bias up to approximately $6000 \mathrm{~kg}$. There is no indication that this trend would continue given a higher number of samples in the upper range of biomass. Alternatively, the uncertainty in the national equations increases with increasing biomass, so our low sample size in larger trees may simply be excluding the lower range of expected biomass for a given diameter.

Much of the consistent negative bias found in the Jenkins et al. equations can be removed with the application of a correction factor (Figure 3.8E). Correction factors for log transformed regression equations are most necessary when uncertainty or RMSE is high, yet the application of this correction is not often made on a consistent basis. In cases of small sample size, correction factors can bias equations further and, considering the paucity of these equations in the literature, correction factors may be frequently applied incorrectly. If an unbiased estimate of biomass is the primary goal, TLS may help determine the forest types and associated existing allometric equations that are most representative and if a correction factor should be applied. Removing additional uncertainty in applying allometry and correctly estimating biomass with low bias will ultimately improve confidence in stand-level biomass assessments.

The TLS allometry deviated from the generalized national equations in both slope and error. The uncertainty of the national allometry encompassed the slope coefficients of nearly all of the TLS equations, with the exception of Quercus rubra (significantly lower) and Quercus prinus (significantly higher). On average, the error for species specific allometry was slightly higher than the national equations, with several equations above and below the mean RMSE, but the inclusion of height and wood density reduced both RMSE variability and mean RMSE below the national equations (Figure 3.9). The reductions in RMSE are an objective benefit of creating high sample size local allometry with TLS. However, is should be clear that lower uncertainty equations are not necessarily indicative of a better, more representative equation. Some lower sample size equations tend to have lower RMSE (e.g. Quercus prinus, $\mathrm{n}=11$, RMSE $=21 \%$ ), but this is more likely a product of insufficient sample size spread evenly over a wide diameter range. In contrast, higher sample size species-specific equations (e.g. Liriodendron tulipifera, 
$\mathrm{n}=48, \mathrm{RMSE}=12 \%$ ) have low RMSE, but more likely represent the range of variability in tree structure. The reported uncertainty of allometric equations with lower sample sizes may not be representative of the local population variability, reinforcing the need for increasing sample size allometry.

\subsection{Conclusion}

TLS is an effective tool for estimating biomass of individual trees and the creation of local allometric equations. We successfully and non-destructively created 40 different empirically derived species-specific relationships predicting height and biomass. The analysis of sample size showed allometry with less than 100 individuals may be unreliable and, if large trees are not included, unrepresentative. The national equations were representative of some species, but underestimated several others. The species specific allometry derived in this study had nearly equivalent or lower RMSE on average compared to the national allometry - a finding suggesting substantial benefits in areas without region- or species-specific equations. Future work should expand the framework used in this study to areas devoid of species specific allometry. Tropical allometry has the potential to be vastly improved, as the most widely applied equations are at the pan-tropical scale. Also, TLS may be one of the best methods for creating full three-dimensional plot reconstructions that can be used for airborne and satellite remote sensing. By adopting the framework described, site-specific allometric equations can be created and expanded to plots without TLS data for reduced uncertainty biomass mapping that could substantially improve estimates of regional and global terrestrial carbon. 


\subsection{Appendix}

Table 3.4: Species-Specific height allometry for the 10 most dominant species at the study site using diameter as the predictor. Blank spaces indicate the same coefficient was used across all species.

\begin{tabular}{llclccccc}
\hline \hline Species & $\mathrm{n}$ & range & $\beta_{0}$ & $\beta_{0}[\mathrm{se}]$ & $\beta_{1}$ & $\beta_{1}[\mathrm{se}]$ & $R^{2}$ & RMSE (\%) \\
\hline Carya cordiformis & 11 & $8-40 \mathrm{~cm}$ & 0.3254 & 0.2808 & 0.8181 & 0.094 & 0.76 & 23.84 \\
Carya glabra & 62 & $4-47 \mathrm{~cm}$ & 0.8369 & 0.2932 & & & 0.89 & 13.87 \\
Carya ovalis & 15 & $6-46 \mathrm{~cm}$ & 0.976 & 0.3652 & & & 0.89 & 12.88 \\
Carya tomentosa & 33 & $5-41 \mathrm{~cm}$ & 0.9557 & 0.3066 & & & 0.8 & 19.8 \\
Fagus grandifolia & 20 & $6-105 \mathrm{~cm}$ & 1.1279 & 0.3189 & & & 0.84 & 15.64 \\
Fraxinus americana & 16 & $8-55 \mathrm{~cm}$ & 0.7768 & 0.3368 & & 0.94 & 10.31 \\
Liriodendron tulipifera & 48 & $10-120 \mathrm{~cm}$ & 1.2637 & 0.3172 & & 0.82 & 7.96 \\
Quercus alba & 25 & $21-76 \mathrm{~cm}$ & 1.9495 & 0.4336 & & & 0.65 & 5.99 \\
Quercus prinus & 11 & $11-50 \mathrm{~cm}$ & 0.57 & 0.4722 & & & 0.87 & 6.35 \\
Quercus rubra & 17 & $13-93 \mathrm{~cm}$ & 2.0623 & 0.3789 & & & 0.79 & 3.66 \\
\hline
\end{tabular}

Table 3.5: Species-Specific volume allometry for the 10 most dominant species at the study site using diameter and height as predictors. Blank spaces indicate the same coefficient was used across all species. Note: the $\beta_{1}$ coefficient is identical to the biomass allometry coefficient.

\begin{tabular}{llllccc}
\hline \hline Species & $\beta_{0}$ & $\beta_{0}[\mathrm{se}]$ & $\beta_{1}$ & $\beta_{1}[\mathrm{se}]$ & $R^{2}$ & RMSE $(\%)$ \\
\hline Carya cordiformis & -9.3544 & 0.1218 & 0.9469 & 0.0108 & 0.93 & 46.34 \\
Carya glabra & -9.3118 & 0.0855 & & & 0.96 & 23.41 \\
Carya ovalis & -9.3461 & 0.1036 & & & 0.97 & 19.85 \\
Carya tomentosa & -9.4061 & 0.0913 & & & 0.97 & 29.57 \\
Fagus grandifolia & -9.2224 & 0.0979 & & & 0.96 & 32.34 \\
Fraxinus americana & -9.3638 & 0.1021 & & & 0.99 & 12.94 \\
Liriodendron tulipifera & -9.3244 & 0.0898 & & & 0.99 & 10.26 \\
Quercus alba & -9.2945 & 0.0978 & & 0.93 & 23.4 \\
Quercus prinus & -9.3732 & 0.1123 & & 0.98 & 21.13 \\
Quercus rubra & -9.3382 & 0.1036 & & & 0.98 & 13.68 \\
\hline
\end{tabular}




\section{References}

Baccini, A., Goetz, SJ, Walker, WS, Laporte, NT, Sun, M, Sulla-Menashe, D, Hackler, J, Beck, PSA, Dubayah, R, Friedl, MA, \& others. 2012. Estimated carbon dioxide emissions from tropical deforestation improved by carbon-density maps. Nature Climate Change, 2(3), 182-185.

Bienert, A., Hess, C., Maas, H.-G., \& von Oheimb, G. 2014. A voxel-based technique to estimate the volume of trees from terrestrial laser scanner data. ISPRS - International Archives of the Photogrammetry, Remote Sensing and Spatial Information Sciences, XL-5(June), 101-106.

Bourg, Norman A., McShea, William J., Thompson, Jonathan R., McGarvey, Jennifer C., \& Shen, Xiaoli. 2013. Initial census, woody seedling, seed rain, and stand structure data for the SCBI SIGEO Large Forest Dynamics Plot: Ecological Archives E094-195. Ecology, 94(9), 2111-2112.

Brown, S. 1997. Estimating biomass and biomass change of tropical forests. A primer.

Calders, Kim, Newnham, Glenn, Burt, Andrew, Murphy, Simon, Raumonen, Pasi, Herold, Martin, Culvenor, Darius, Avitabile, Valerio, Disney, Mathias, Armston, John, \& Kaasalainen, Mikko. 2015. Nondestructive estimates of above-ground biomass using terrestrial laser scanning. Methods in Ecology and Evolution, 6(2), 198-208.

Chave, Jerome, Condit, Richard, Aguilar, Salomon, Hernandez, Andres, Lao, Suzanne, \& Perez, Rolando. 2004. Error propagation and scaling for tropical forest biomass estimates. Philosophical Transactions of the Royal Society B: Biological Sciences, 359(1443), 409-420.

Chave, Jrme, Muller-Landau, Helene C., Baker, Timothy R., Easdale, Toms A., Steege, Hans ter, \& Webb, Campbell O. 2006. Regional and phylogenetic variation of wood density across 2456 neotropical tree species. Ecological applications, 16(6), 2356-2367. 
Chen, Qi, Vaglio Laurin, Gaia, \& Valentini, Riccardo. 2015. Uncertainty of remotely sensed aboveground biomass over an African tropical forest: Propagating errors from trees to plots to pixels. Remote Sensing of Environment, 160(Apr.), $134-143$.

Chojnacky, D. C., Heath, L. S., \& Jenkins, Jennifer C. 2014. Updated generalized biomass equations for North American tree species. Forestry, 87(1), 129-151.

Duncanson, L., Rourke, O., \& Dubayah, R. 2015a. Small Sample Sizes Yield Biased Allometric Equations in Temperate Forests. Scientific Reports, 5(Nov.), 17153.

Duncanson, Laura I., Dubayah, Ralph O., \& Enquist, Brian J. 2015b. Assessing the general patterns of forest structure: quantifying tree and forest allometric scaling relationships in the United States: Forest allometric variability in the United States. Global Ecology and Biogeography, 24(12), 1465-1475.

Flewelling, James W., \& Pienaar, L. V. 1981. Multiplicative Regression with Lognormal Errors. Forest Science, 27(2), 281-289.

Hackenberg, Jan, Morhart, Christopher, Sheppard, Jonathan, Spiecker, Heinrich, \& Disney, Mathias. 2014. Highly Accurate Tree Models Derived from Terrestrial Laser Scan Data: A Method Description. Forests, 5(5), 1069-1105.

Hackenberg, Jan, Wassenberg, Marc, Spiecker, Heinrich, \& Sun, Dongjing. 2015. Non destructive method for biomass prediction combining TLS derived tree volume and wood density. Forests, 6(4), 1274-1300.

Hopkinson, Chris, Chasmer, Laura, Young-Pow, Colin, \& Treitz, Paul. 2004. Assessing forest metrics with a ground-based scanning lidar. Canadian Journal of Forest Research, 34(3), 573-583.

Hosoi, Fumiki, Nakai, Yohei, \& Omasa, Kenji. 2013. 3-D voxel-based solid modeling of a broad-leaved tree for accurate volume estimation using portable scanning lidar. ISPRS Journal of Photogrammetry and Remote Sensing, 82(Aug.), 41-48.

Houghton, R. A., Hall, Forrest, \& Goetz, Scott J. 2009. Importance of biomass in the global carbon cycle. Journal of Geophysical Research-Biogeosciences, 114(Sept.), G00E03.

Jenkins, J. C., Chojnacky, David C., Heath, Linda S., \& Birdsey, Richard A. 2003. National-scale biomass estimators for United States tree species. Forest Science, 49(1), 12-35. 
Jenkins, Jennifer C., Birdsey, Richard A., \& Pan, Yude. 2001. Biomass and Npp Estimation for the Mid-Atlantic Region (usa) Using Plot-Level Forest Inventory Data. Ecological Applications, 11(4), 1174-1193.

Jucker, Tommaso, Caspersen, John, Chave, Jrme, Antin, Ccile, Barbier, Nicolas, Bongers, Frans, Dalponte, Michele, van Ewijk, Karin Y., Forrester, David I., Haeni, Matthias, Higgins, Steven I., Holdaway, Robert J., Iida, Yoshiko, Lorimer, Craig, Marshall, Peter L., Momo, Stphane, Moncrieff, Glenn R., Ploton, Pierre, Poorter, Lourens, Rahman, Kassim Abd, Schlund, Michael, Sonk, Bonaventure, Sterck, Frank J., Trugman, Anna T., Usoltsev, Vladimir A., Vanderwel, Mark C., Waldner, Peter, Wedeux, Beatrice M. M., Wirth, Christian, Wll, Hannsjrg, Woods, Murray, Xiang, Wenhua, Zimmermann, Niklaus E., \& Coomes, David A. 2017. Allometric equations for integrating remote sensing imagery into forest monitoring programmes. Global Change Biology, 23(1), 177190.

Kankare, Ville, Holopainen, Markus, Vastaranta, Mikko, Puttonen, Eetu, Yu, Xiaowei, Hyypp, Juha, Vaaja, Matti, Hyypp, Hannu, \& Alho, Petteri. 2013. Individual tree biomass estimation using terrestrial laser scanning. ISPRS Journal of Photogrammetry and Remote Sensing, 75(Jan.), 64-75.

Mitchard, Edward T. A., Feldpausch, Ted R., Brienen, Roel J. W., LopezGonzalez, Gabriela, Monteagudo, Abel, Baker, Timothy R., Lewis, Simon L., Lloyd, Jon, Quesada, Carlos A., Gloor, Manuel, ter Steege, Hans, Meir, Patrick, Alvarez, Esteban, Araujo-Murakami, Alejandro, Aragao, Luiz E. O. C., Arroyo, Luzmila, Aymard, Gerardo, Banki, Olaf, Bonal, Damien, Brown, Sandra, Brown, Foster I., Ceron, Carlos E., Chama Moscoso, Victor, Chave, Jerome, Comiskey, James A., Cornejo, Fernando, Corrales Medina, Massiel, Da Costa, Lola, Costa, Flavia R. C., Di Fiore, Anthony, Domingues, Tomas F., Erwin, Terry L., Frederickson, Todd, Higuchi, Niro, Honorio Coronado, Euridice N., Killeen, Tim J., Laurance, William F., Levis, Carolina, Magnusson, William E., Marimon, Beatriz S., Marimon Junior, Ben Hur, Mendoza Polo, Irina, Mishra, Piyush, Nascimento, Marcelo T., Neill, David, Nunez Vargas, Mario P., Palacios, Walter A., Parada, Alexander, Pardo Molina, Guido, Pena-Claros, Marielos, Pitman, Nigel, Peres, Carlos A., Poorter, Lourens, Prieto, Adriana, RamirezAngulo, Hirma, Restrepo Correa, Zorayda, Roopsind, Anand, Roucoux, Katherine H., Rudas, Agustin, Salomao, Rafael P., Schietti, Juliana, Silveira, Marcos, de Souza, Priscila F., Steininger, Marc K., Stropp, Juliana, Terborgh, John, Thomas, Raquel, Toledo, Marisol, Torres-Lezama, Armando, van Andel, Tinde R., van der Heijden, Geertje M. F., Vieira, Ima C. G., Vieira, Simone, Vilanova-Torre, Emilio, Vos, Vincent A., Wang, Ophelia, Zartman, Charles E., Malhi, Yadvinder, \& Phillips, Oliver L. 2014. Markedly divergent estimates of 
Amazon forest carbon density from ground plots and satellites. Global Ecology and Biogeography, 23(8), 935-946.

Othmani, Ahlem, Piboule, Alexandre, Krebs, M., Stolz, C., \& Voon, LFC Lew Yan. 2011. Towards automated and operational forest inventories with T-Lidar. In: 11th International Conference on LiDAR Applications for Assessing Forest Ecosystems (SilviLaser 2011).

Picard, Nicolas, Saint-Andre, Laurent, \& Henry, Matieu. 2012. Manual for building tree volume and biomass allometric equations: from field measurement to prediction. Food and Agricultural Organization of the United Nations and Centre de Coopration Internationale en Recherche Agronomique pour le Dveloppement.

Raumonen, Pasi, Kaasalainen, Mikko, kerblom, Markku, Kaasalainen, Sanna, Kaartinen, Harri, Vastaranta, Mikko, Holopainen, Markus, Disney, Mathias, \& Lewis, Philip. 2013. Fast Automatic Precision Tree Models from Terrestrial Laser Scanner Data. Remote Sensing, 5(2), 491-520.

Saatchi, Sassan, Harris, Nancy L., Brown, Sandra, Lefsky, Michael, Mitchard, Edward T. A., Salas, William, Zutta, Brian R., Buermann, Wolfgang, Lewis, Simon L., Hagen, Stephen, Petrova, Silvia, White, Lee, Silman, Miles, \& Morel, Alexandra. 2011. Benchmark map of forest carbon stocks in tropical regions across three continents. Proceedings of the National Academy of Sciences of the United States of America, 108(24), 9899-9904.

Seidel, Dominik, Albert, Katja, Ammer, Christian, Fehrmann, Lutz, \& Kleinn, Christoph. 2013. Using terrestrial laser scanning to support biomass estimation in densely stocked young tree plantations. International Journal of Remote Sensing, 34(24), 8699-8709.

Sheridan, Ryan D. 2011. Modeling Plot-Level Biomass and Volume Using Airborne and Terrestrial Lidar Measurements. Master's thesis, Texas A\&M University. Available electronically from http://hdl. handle. net/1969, 1.

Sprugel, D. G. 1983. Correcting for Bias in Log-Transformed Allometric Equations. Ecology, 64(1), 209-210.

Srinivasan, Shruthi, Popescu, Sorin C., Eriksson, Marian, Sheridan, Ryan D., \& $\mathrm{Ku}$, Nian-Wei. 2014. Multi-temporal terrestrial laser scanning for modeling tree biomass change. Forest Ecology and Management, 318(Apr.), 304-317.

TerMikaelian, M. T., \& Korzukhin, M. D. 1997. Biomass equations for sixty-five North American tree species. Forest Ecology and Management, 97(1), 1-24. 
Wilkes, Phil, Lau, Alvaro, Disney, Mathias, Calders, Kim, Burt, Andrew, Gonzalez de?Tanago, Jose, Bartholomeus, Harm, Brede, Benjamin, \& Herold, Martin. 2017. Data acquisition considerations for Terrestrial Laser Scanning of forest plots. Remote Sensing of Environment, 196(July), 140-153.

Yao, Tian, Yang, Xiaoyuan, Zhao, Feng, Wang, Zhuosen, Zhang, Qingling, Jupp, David, Lovell, Jenny, Culvenor, Darius, Newnham, Glenn, Ni-Meister, Wenge, Schaaf, Crystal, Woodcock, Curtis, Wang, Jindi, Li, Xiaowen, \& Strahler, Alan. 2011. Measuring forest structure and biomass in New England forest stands using Echidna ground-based lidar. Remote Sensing of Environment, 115(11), 2965-2974. 


\section{Chapter 4}

Improved Biomass Calibration with Terrestrial LiDAR

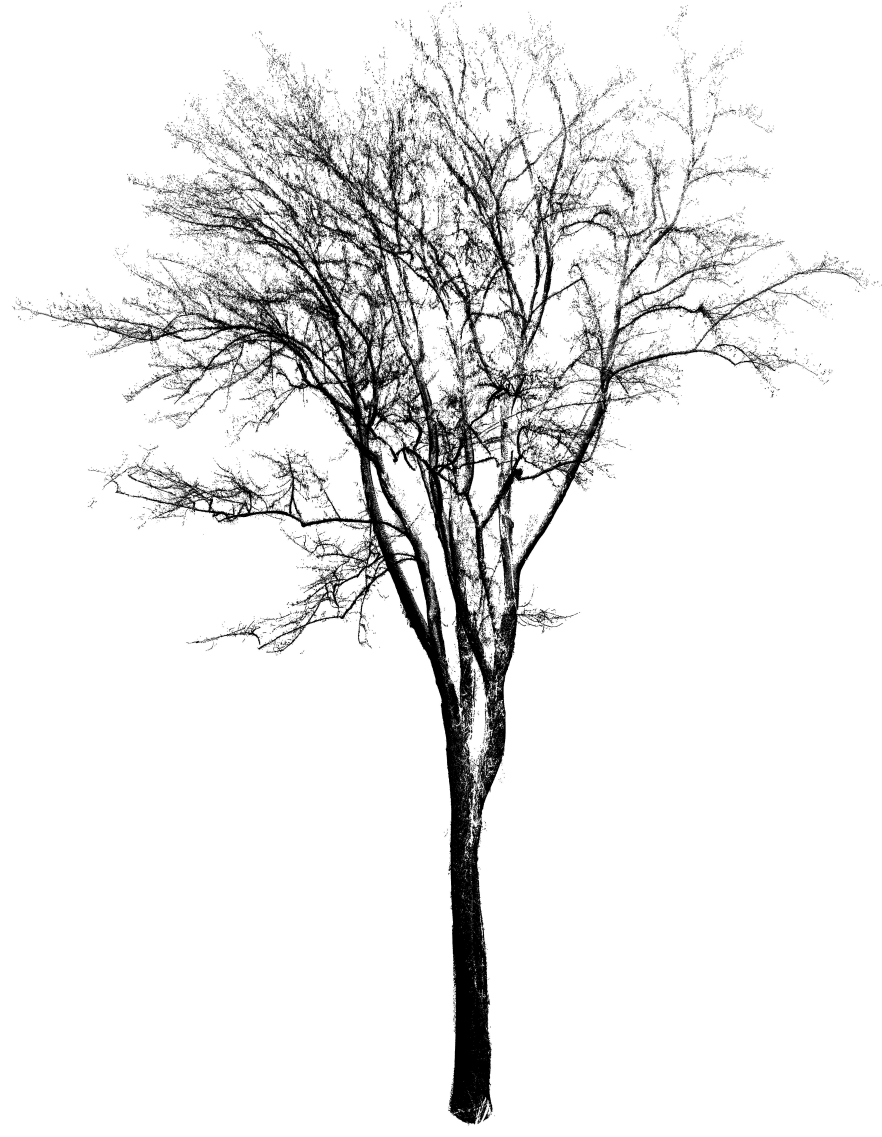




\section{Abstract}

Future NASA and ESA satellite missions plan to better quantify global carbon stocks through detailed observations of forest structure, but ultimately rely on uncertain ground measurement approaches for calibration and validation. A significant amount of this uncertainty in estimating plot-level biomass can be attributed to inadequate and unrepresentative allometric relationships used to convert plotlevel tree measurements to estimates of aboveground biomass. These allometric equations are known to have high errors and biases, particularly in carbon rich forests, because they were calibrated with small and often biased samples of destructively harvested trees. To overcome this issue, a non-destructive methodology for estimating tree and plot-level biomass has been proposed through the use of Terrestrial Laser Scanning (TLS). We investigated the potential for using TLS as a ground validation approach in LiDAR-based biomass mapping though virtual plot-level tree volume reconstruction and biomass estimation. Plot-level biomass estimates were compared in the Virginia-based Smithsonian Conservation Biology Institute's Large Forest Dynamics Plot (LFDP) with full 3D reconstruction, TLS allometry, and Jenkins et al. (2003) allometry. On average, full 3D reconstruction ultimately provided the lowest uncertainty estimate of plot-level biomass (14.4\%), followed by TLS allometry (17.9\%) and the national equations (18.5\%). TLS reduced error in the airborne LiDAR empirical models by over $10 \%$. Our findings suggest TLS plot acquisitions and non-destructive allometry can play a vital role in reducing uncertainty of calibration and validation data for biomass mapping in the upcoming NASA and ESA missions. ${ }^{1}$

\footnotetext{
${ }^{1}$ This chapter is based on: Stovall, A.E.L., Shugart, H.H., In Review. Improved Biomass Calibration and Validation with Terrestrial LiDAR: Implications for Future LiDAR and SAR Missions. intended for IEEE JSTARS.
} 


\subsection{Introduction}

Forests provide essential ecosystem services and hold the vast majority of terrestrial carbon, but remain the most uncertain components of the carbon cycle (Houghton et al., 2009). Efforts to quantify the massive and dynamic storage of global carbon with a higher degree of certainty have revealed discrepancies arising from differing approaches (Saatchi et al., 2011; Baccini et al., 2012). Disagreement in the magnitude of these distributions emphasizes the weak link in carbon mapping stems from individual tree and aggregated plot-level biomass estimates that are not representative (Mitchard et al., 2014). Biomass estimates are derived from equations built from laborious destructive sampling of individual trees (Picard et al., 2012). The inherent difficulty surrounding destructive harvesting of trees leads to insufficient sample sizes that produce unrepresentative, spatially biased equations, rarely including large trees (Chave et al., 2004). Before global carbon mapping can be improved, substantial progress must be made in creating more representative allometry that will improve the accuracy of plot level estimates of biomass.

Three-dimensional forest structure quantification is required for effective ecosystem service management and understanding current and future global carbon dynamics (Houghton et al., 2009). Global datasets capturing cover at coarse (MODIS) and fine (Landsat) spatial scales have allowed us to monitor forest status, but global information on 3D structure is insufficient for fine scale management of essential ecosystem services (Hall et al., 2011). Accurate mapping of more complex ecosystem characteristics such as biomass requires higher spatial resolution and information on forest structure (Wickland et al., 2014). Globally-distributed highresolution three-dimensional data will provide the necessary information for managing ecosystems and constraining future climate projections (Goetz \& Dubayah, 2011).

Airborne sensors are often used to calibrate spaceborne sensors, but accuracy is dependent on quality plot-level biomass estimates. NASAs Land, Vegetation, and Ice Sensor (LVIS) is capable of capturing fine-scale three-dimensional vegetation structure that can simulate GEDI (Global Ecosystem Dynamics Investigation) and ICESAT-2 (Ice, Cloud, and land Elevation Satellite) data, allowing investigation of 
the instrument-specific response in a range of forest ecosystems prior to mission launch. Similarly, the synthetic aperture radar (SAR) used on UAVSAR can inform calibration of spaceborne pol-InSAR, such as NI-SAR (NASA-ISRO SAR) or the European Space Agencys BIOMASS mission. The success of these missions is directly dependent on accurate plot-level estimates of biomass that will ultimately improve confidence in landscape scale mapping. Precise and unbiased estimates of biomass at the plot level are an essential component to significantly improving carbon estimation with all future missions estimating global carbon. However, errors in plot-level biomass from unrepresentative allometry are unavoidable when relying on traditional field measurements for calibration and validation.

The empirical relationships used to relate tree diameter and height to biomass, or allometry, are known to be spatially variable (Duncanson et al., 2015c) and not representative at low sample sizes (Duncanson et al., 2015a; Chave et al., 2004). The solution to high variability in allometry has been the pooling of massive datasets globally to create stable equations that are not species specific (Chave et al., 2014). While this approach is acceptable for coarse estimates of forest biomass it is insufficient for calibration and validation plot-level data for GEDI and other missions as they must have high accuracy and precision because non species specific equations can have greater than 350\% error (McRoberts et al., 2016), emphasizing the importance of equation selection (Zhao et al., 2012). Improvement in allometric equations is one way to significantly improve calibration and validation plot data since it does not require resampling and can be applied across large areas, but the inherent difficulties surrounding destructive sampling limits the feasibility of this approach. Efficient, automated, and non-destructive methods of estimating aboveground biomass of single trees, such as terrestrial LiDAR, offers a solution to the problems of unrepresentative allometry.

Terrestrial Laser Scanning (TLS) or terrestrial LiDAR is the best current method of non-destructively estimating single tree and plot-level biomass in a range of ecosystems (Calders et al., 2015; Stovall et al., 2017). TLS recreates plot level structure with millimeter detail, allowing for three-dimensional reconstruction of tree stems with geometric modeling (Raumonen et al., 2013). TLS has been deployed in most forest ecosystem types, successfully estimating tree volume and biomass with lower error than allometric equations (Calders et al., 
2015; Hackenberg et al., 2015). Calibration and validation plot data can be significantly improved by applying TLS as a tool for estimating plot-level biomass with higher accuracy than allometric equations. Moreover, non-destructive tree modeling with TLS offers the potential for unbiased and high sample size allometry that can include trees of any size with minimal effort. The greatest improvements to calibration and validation data are surely to come in tropical forests where regionspecific and species-specific allometries are extremely rare. While deploying TLS for direct plot-level biomass estimation and the creation of area-specific allometry will likely reduce uncertainty in plot level biomass estimates used for calibration and validation it is still unclear whether or not this will result in major differences at the level of sensor calibration.

This study presents terrestrial laser scanning as a potential approach to improving airborne and satellite-based biomass calibration and validation through direct structural measurements and improved allometric equation development (Figure 4.1). We investigate TLS in this context by (1) directly modeling plot-level biomass and developing high sample size non-destructive local biomass allometry for the dominant species groupings in a broadleaf deciduous hardwood forest, (2) using both direct and allometrically-derived TLS biomass estimates for calibrating an empirical plot biomass model with LiDAR, and (3) comparing the uncertainty of the biomass models to an equivalent approach relying on the national-scale Jenkins et al. (Jenkins et al., 2003) biomass allometry.

\subsection{Methods}

\subsubsection{Study Area and Species Selection}

The study site is the 25.6 ha (400 m x 600 m) Smithsonian Institute Global Earth Observatory (SIGEO) temperate Large Forest Dynamics Plot in the Smithsonian Conservation Biology Institute near Front Royal, VA (3853'36.6” N, 788'43.4" W; Fig 1). This mixed deciduous hardwood forest is nearly 100 years old and representative of many on the east coast experiencing post-agricultural regrowth. Elevation ranges from 273-338 $\mathrm{m}$ and mean annual temperature and precipitation for the area is $12.7 \mathrm{C}^{\circ}$ and $970 \mathrm{~mm}$ respectively. This SIGEO site was inten- 
(A)

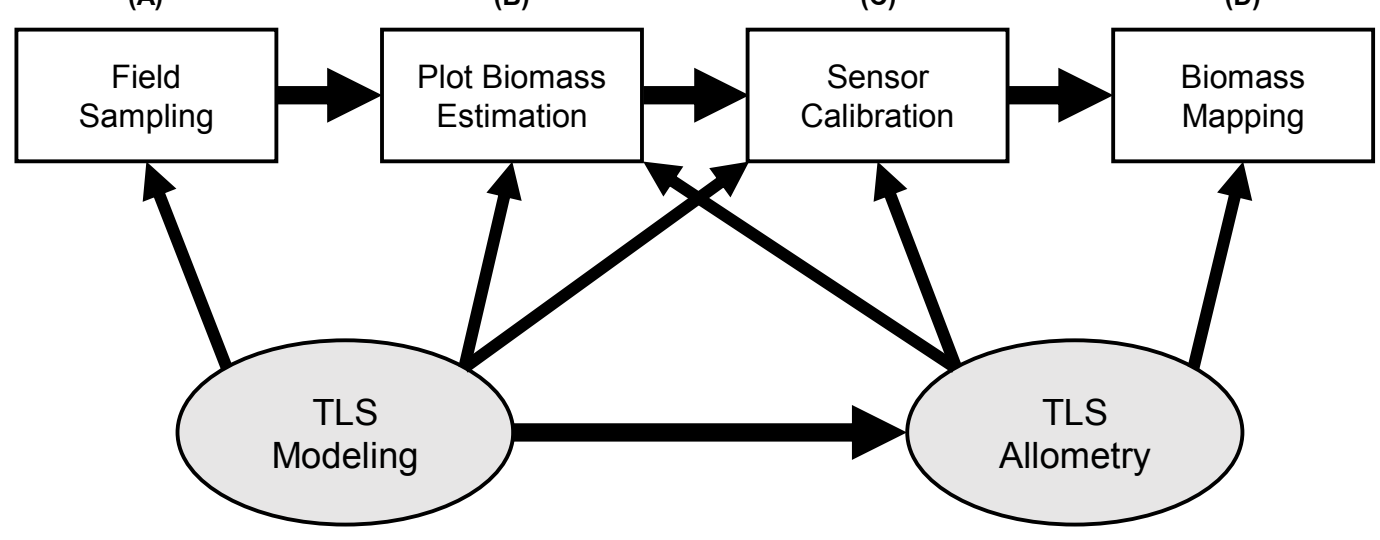

Figure 4.1: Framework for reducing uncertainty with targeted TLS acquisition through modeling and improved allometry. At each step of biomass mapping TLS can potentially improve the current methodology. (A) At the stage of field sampling TLS is an efficient and relatively unbiased method of collecting standard forest structure information (e.g. DBH, height, basal area, vegetation area index). (B) Plot-level biomass estimation can be improved through direct stem modeling with TLS or non-destructive local allometry with TLS. (C) TLS can potentially improve sensor calibration and validation through full plot-level 3D reconstruction. Reduced uncertainty biomass allometry can be applied regionally, further improving sensor calibration. (D) Low uncertainty sensor calibration will provide the greatest potential for reducing uncertainty in biomass mapping.

sively sampled in 2010 for species, diameter, and stem location from a data set of over 56,000 individuals greater than $1 \mathrm{~cm}$ diameter at breast height (DBH) (Bourg et al., 2013). We used the a priori forest structure information to determine the most important species for biomass analysis based on available allometry. The dominant species contributing biomass to the forest with average wood density values $\left(\mathrm{g} / \mathrm{cm}^{3}\right)$ were: Liriodendron tulipifera (0.40), Carya cordiformis (0.62), Carya glabra (0.62), Carya ovalis (0.62), Carya tomentosa (0.62), Fagus grandifolia (0.56), Fraxinus americana (0.55), Nyssa sylvatica (0.46), Quercus alba (0.60), Quercus prinus (0.57), Quercus rubra (0.56), and Quercus velutina (0.56) (Chojnacky et al., 2014). These species were used for non-destructive TLS-based local allometric equation development described in the subsequent sections, as they were 
estimated to contribute over $80 \%$ of aboveground forest biomass.

\subsubsection{TLS Allometry}

\section{TLS Sampling and Post Processing}

Within the SIGEO forest, we chose $141 / 10$ th ha circular (radius $\approx 17.8 \mathrm{~m}$ ) plot sampling locations at random using ArcGIS mapping software. TLS sampling took place over 4 days in March and April of 2015 during leaf-off conditions. Individual plots were located using a handheld Garmin eTrex GPS unit and plot centers were found on the marked $20 \mathrm{~m}$ grid intersections of the SIGEO plot grid network. The Faro Focus 120 3D phase-shift TLS was set to medium resolution and quality (1/5 resolution and $4 \mathrm{x}$ quality) for a total of 28.2 million pulses per scan. Time elapsed per scan was approximately 3 minutes. We reduced occlusion from the presence of high-density vegetation by scanning 5 times in a diamond pattern oriented at approximately each cardinal direction to provide sufficient coverage and a standardized sampling scheme. At times, an additional scan was required for full coverage. To aid in post-processing scan registration we placed 6-inch diameter polystyrene spheres atop fiberglass stakes throughout the plot. Multiple scans were digitally registered using the registration points, as described in the following section.

\section{Post Processing}

Individual scans were registered using the automatic registration algorithms included in Faro SCENE (SCENE (version 5.4.4.41689), 2015) and overlapping redundant points were filtered to create a single 3D point cloud used for modeling. The registration process relied on the spheres used on each field plot. Spheres were located in every scan and aligned with each corresponding sphere in other scans from the same plot. We evaluated the registration error within SCENE (typical registration error did not exceed $1 \mathrm{~cm}$ ). Once the registration was completed with an acceptably low placement error, the data was filtered removing all returns below the intensity threshold of 400. A stray point filter included in the Faro software was then used to remove ambiguous points at the edges of vegetation. The fully 


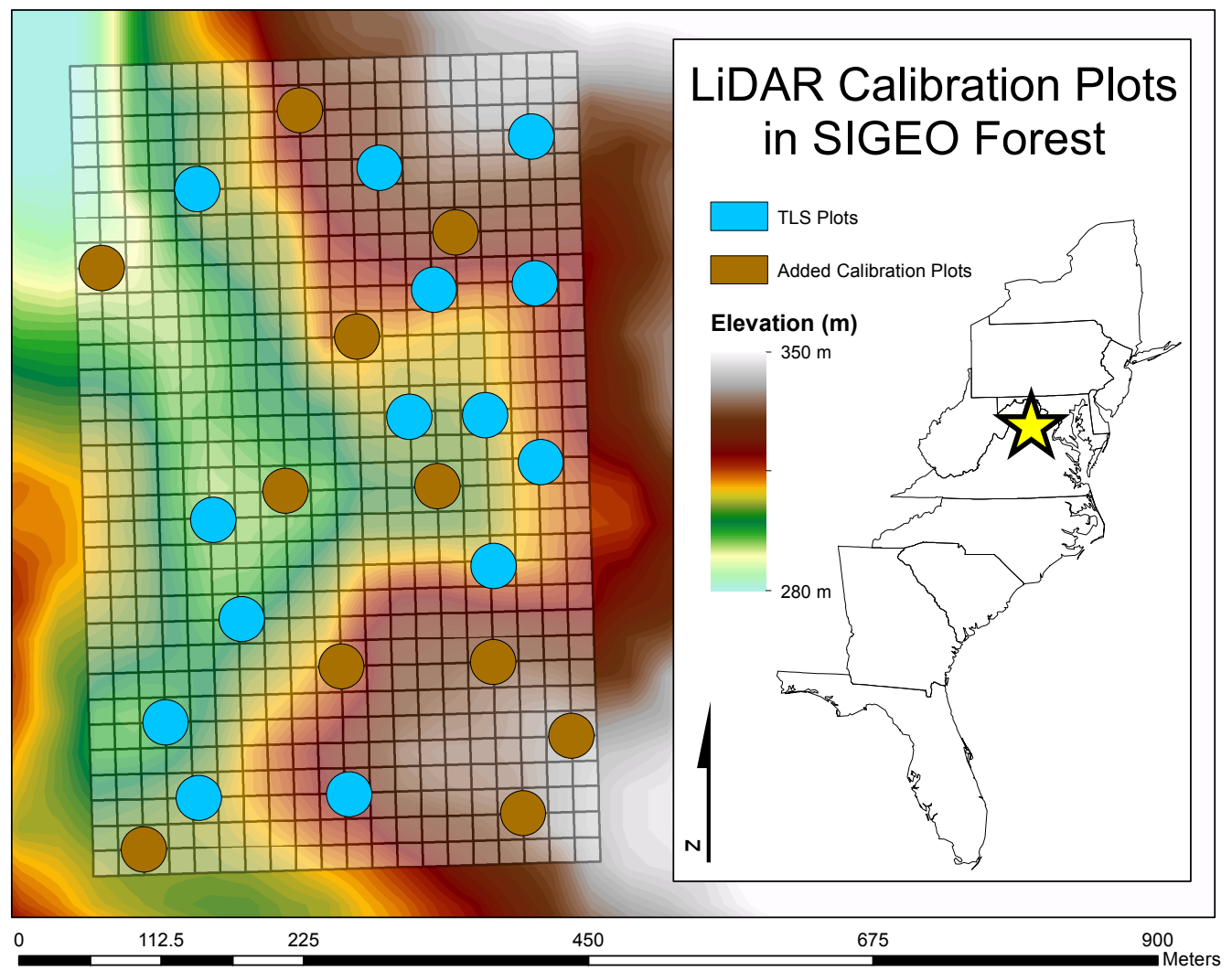

Figure 4.2: Overview map of SIGEO forest at the Smithsonian Conservation Biology Institute in Front Royal,VA ( $\star$ ). 14 randomly distributed 1/10th ha circular plots were located on the $20 \mathrm{~m}$ grid intersections and scanned with TLS (blue). 11 additional plots were included for LiDAR calibration (brown). An elevation model derived from the 2011 LiDAR acquisition shows the topography across the site. Note: TLS plots are not always centered over grid intersections as the TLS point cloud was georeferenced with the airborne LiDAR.

registered point cloud data was then exported with intensity values, column and row numbers, which correspond to scan azimuth and angle, as well as intensity values ranging from 0 to 2100. Registered plot-level point clouds typically had approximately 100 million returns in closed canopy forest. 


\section{Volume and Biomass Estimation}

We separated and modeled individual trees on each plot using an automated work flow within the CompuTree software (Othmani et al., 2011). The processing took place in four steps: (1) ground point classification and DTM creation, (2) stem identification, (3) tree segmentation, and (4) stem reconstruction with quantitative structure models (QSMs). The ground points were classified with a local minima ground estimation algorithm, which reconstructs the DTM, while excluding aboveground points associated with vegetation. Stems were identified using a nearest neighbor and connected components approach on a small slice in the point cloud made parallel to the DTM. Stems spaced apart from one another were identified as a unique object and this portion of the point serves are the initial seed point to initiate the cylinder modeling algorithm implemented in SimpleTree (Hackenberg et al., 2015). The tree was then automatically segmented from the point cloud using an iterative nearest neighbor approach, starting at the initial seed point on the stem and moving vertically while expanding in area with the expanding tree crown. The segmented tree was then reconstructed through cylinder fitting with the QSM algorithm. The best fit cylinders were used as a guide for creating an allometric relationship between trunk size and branching order - an adaptation of the pipe model concept or scaling theory (West et al., 1999). This single-tree relationship guided all low certainty cylinder measurements, filling gaps in the tree model with likely cylinder sizes that correspond to the expected branching order. This approach as implemented in SimpleTree was validated with a number of destructive samples and accurately estimated biomass with approximately 10-15\% RMSE (Hackenberg et al., 2015). Based on the previous success of these algorithms in similar forest systems in leaf-off conditions, we anticipated this application in our forest to be comparable in accuracy.

The cylinder models (referred to as TLS models hereafter) provided an estimate of volume, which were converted to estimates of biomass using:

$$
\text { Biomass }=\rho_{s} V_{T L S}
$$

where $V_{T L S}$ is modeled volume $\left(\mathrm{m}^{3}\right)$ and $\rho_{s}$ is average species specific wood density $\left(\mathrm{g} / \mathrm{cm}^{3}\right)$. Biomass estimates were converted to $\mathrm{kg}$ for direct comparison 
to other allometric equations. While this approach is unable to directly estimate wood density, small scale biomass mapping is unlikely to show wide variation in species-specific wood density. Moreover, the disentanglement of density effects may be beneficial to future national-scale biomass estimation, allowing densitydependent allometry to be used across large areas.

\section{Allometric Equation Development}

Allometric relationships were developed from the TLS biomass estimates with a log-linear regression method taking the form:

$$
\hat{B}_{\text {tree }}=\exp \left(\beta_{0}+\beta_{1} * \ln (D B H)\right)+\epsilon_{\text {tree }}
$$

where $\beta_{0}$ and $\beta_{1}$ are the model coefficients and $\epsilon_{\text {tree }}$ is the residual error from the allometric equation. Modeled trees were grouped into 5 different equations: Liriodendron, Carya, Quercus, Mixed Hardwood, and Maple/Oak/Hickory/Beech (Table 4.2). The groups were based on the dominant forest species (Poplar, Oak, and Hickory) and two general equations similar to groupings in Jenkins et al. that could describe the remaining abundant species. TLS models of Carya species had a maximum diameter of $47 \mathrm{~cm}$, so the Maple/Oak/Hickory/Beech equation was used for individuals that exceeded the Carya allometry diameter range. The high biomass contribution of the 10 species included in these allometric models increases the likelihood for changes to allometrically-derived forest biomass under differing equations.

Several equation forms were evaluated in terms of coefficient of determination $\left(\mathrm{R}^{2}\right)$, Akakai Information Criterion (AIC) weights, and RMSE. While diameter did not explain as much biomass variability as equations also including height, the diameter only equation provided a clear comparison to the Jenkins et al. equations. Model uncertainty of each allometric equation was reported in terms of RMSE in log units in order to reflect increasing uncertainty with increasing biomass and to directly compare to the Jenkins et al. equations reported error. We performed a 5 -fold cross validation on each allometric equation to independently estimate uncertainty. 


\section{Biomass Estimation}

We applied our non-destructive allometry and Jenkins et al. allometry to the diameter measurements of the trees located within each TLS plot. For those individuals that were not selected for updated allometry, the national equations were used in order to provide a biomass contribution in our assessment. We created biomass maps from (1) TLS models with TLS allometry, (2) TLS allometry, and (3) Jenkins et al. allometry. The plot-level biomass estimates derived from the direct three-dimensional tree models were supplemented with TLS allometry or national scale allometry if the individual was not successfully modeled, as was the case with many small trees in each plot. The national scale equations were substituted if the species was not one of the ten species included in the TLS allometry to ensure every individual contributed biomass regardless of the equation used. Aboveground biomass density $\left(\mathrm{Mg} \mathrm{ha}^{-1}\right)$ was estimated by aggregating tree estimates to the plot-level using:

$$
\hat{B}_{\text {plot }}=\sum_{i=1}^{n_{\text {tree }, \text { plot }}} \hat{B}_{\text {tree }, i} / s
$$

where $n_{\text {tree,plot }}$ is the number of trees on a plot and $s$ is the area of the plot in hectares. We propagate uncertainty due to allometry to the plot level with methods outlined in a previous study (Chen et al., 2015) using:

$$
\sigma_{\text {plot }}=\sqrt{\sum_{i=1}^{n_{\text {tree } p l o t}} \sigma_{\text {tree }, i^{2}} / s}
$$

where $\sigma_{\text {tree }, i}$ is the biomass error from allometric equation residuals.

\subsubsection{LiDAR Biomass Calibration and Validation}

\section{Airborne LiDAR Data Sources}

Airborne LiDAR data was acquired with the Optech 3100 instrument at approximately $1371 \mathrm{~m}$ above ground level over an area overlapping the SIGEO forest from March 1-9, 2011 and extended nearly 2,500 km² beyond our site. Nominal point spacing was $1 \mathrm{~m}$ and up to 4 returns were recorded. The primary purpose 
of the LiDAR acquisition was high resolution DEM creation, so it was completed in leaf-off conditions at this lower point spacing. Average horizontal error was 37 $\mathrm{cm}$.

\section{LiDAR Canopy Metrics}

Canopy height and LiDAR intensity were used as two analogous variables to the upcoming missions attempting to characterize global forest structure. Canopy height will be captured with LiDAR (GEDI and ICE-SAT II) and PolInSAR or TomoSAR (BIOMASS and NISAR), thus making this structural variable essential for estimating biomass and quantifying the potential for uncertainty reduction with application of TLS. While LiDAR intensity is not a direct representation of radar backscatter, both metrics describe signal intensity and are derived from the same basic principal as defined in the radar equation (Wagner et al., 2006). As such, intensity may provide supplementary structural information not captured with height metrics alone in the case of airborne LiDAR, while providing insight for future applications to PolSAR and TomoSAR. The combination of the height and LiDAR intensity may provide the best approximation of forest biomass as structure and top-of-canopy reflectance or texture are both characterized in a single empirical model of biomass.

We used the Fusion LiDAR Data Toolkit (LDK) to calculate mean canopy height $(\mathrm{MCH})$ and LiDAR intensity (I) at a $30 \mathrm{~m}$ resolution. The processing was accomplished using the CloudMetrics algorithm. Mean canopy height was calculated by first creating a $5 \mathrm{~m}$ resolution digital earth model (DEM) using all LiDAR ground returns. The LiDAR data was normalized to the DEM, subtracting the corresponding DEM elevation from the point cloud. Ground returns were removed with a simple $1 \mathrm{~m}$ height threshold above the DEM. Mean canopy height was calculated as the mean height above the DEM of all returns above ground. The $99^{\text {th }}$ percentile of intensity was selected as the maximum intensity metric is sensitive to abnormally high energy returns. 


\section{LiDAR Empirical Models}

Three empirical model forms were used to estimate plot biomass relying on single linear regression for the individual LiDAR metrics - MCH and intensity - and multiple linear regression for the combination of the two metrics. The three models were created for each set of biomass estimation methods: (1) TLS models, (2) TLS allometry, and (3) Jenkins et al. allometry. The combination compared the three LiDAR-based metrics and three methods of estimating biomass, for a total of 9 empirical models. For each simple linear model, biomass $\left(\hat{B}_{\text {plot }}\right)$ was estimated using:

$$
\hat{B}_{p l o t}=\beta_{0}+\beta_{1}(M C H, I)+\epsilon_{p l o t}
$$

where $\beta_{0}$ and $\beta_{1}$ are the model coefficients describing the relationship of LiDAR metrics (MCH or intensity) to biomass. The error term $\epsilon_{\text {plot }}$ represents the residual error of the empirical biomass model. In the case of multiple regression the model takes the form:

$$
\hat{B}_{\text {plot }}=\beta_{0}+\beta_{1}(M C H)+\beta_{2}(I)+\epsilon_{\text {plot }}
$$

where $\beta_{1}$ and $\beta_{2}$ are coefficients for $\mathrm{MCH}$ and intensity respectively. $\epsilon_{\text {plot }}$ is entirely derived from residual error, however, given these formulations, plot biomass uncertainty is not accounted for. Any variation in $\epsilon_{\text {plot }}$ under differing allometric relationships will be due to changes in mean biomass density, rather than propagated allometric uncertainty. We quantify LiDAR model prediction uncertainty including allometric error with:

$$
\sigma_{\text {pred }}^{2}=\sigma_{\epsilon, \text { plot }}^{2}+\sigma_{\epsilon, \hat{B}_{\text {plot }}}^{2}
$$

where $\sigma_{\text {pred }}$ is total uncertainty from the empirical biomass model, $\sigma_{\epsilon, p l o t}$ is uncertainty due to LiDAR model residuals, and $\sigma_{\epsilon, \hat{B}_{p l o t}}$ is uncertainty propagated from allometric equations to the plot level. The uncertainty from the sources described were found to substantially contribute to biomass prediction uncertainty in a similar analysis that quantified most sources of uncertainty in biomass mapping (Chen et al., 2015). 
Table 4.1: Summary statistics for all trees modeled for TLS allometry. $\rho$ is based on the average wood density and not independently derived.

\begin{tabular}{lccccc}
\hline \hline Statistic & N & Mean & St. Dev. & Min & Max \\
\hline$\rho$ & 243 & 0.548 & 0.083 & 0.400 & 0.620 \\
DBH $(\mathrm{cm})$ & & 34.6 & 20.4 & 10.1 & 120.0 \\
Height $(\mathrm{m})$ & 22.80 & 7.56 & 7.11 & 36.70 \\
Volume $\left(\mathrm{m}^{3}\right)$ & 2.33 & 3.21 & 0.03 & 25.20 \\
Biomass $(\mathrm{kg})$ & 1,207 & 1,584 & 18 & 11,179 \\
\hline
\end{tabular}

\subsection{Results}

\subsubsection{TLS Allometry}

TLS successfully created 5 different allometric equations using 243 3D tree models (Table I). Small stems were difficult to accurately model so most were excluded in this study, but considering our interest in estimating biomass we measured TLS modeling success by total basal area captured per plot. On average, TLS modeled $70.6 \%$ of plot basal area. A single plot was a strong outlier with extremely dense understory vegetation and only three trees successfully modeled with TLS. Removing this outlier increased total plot basal area modeled with TLS to $92.9 \%$. From the TLS models, three equations were created according to the dominant species across the site: Liriodendron tulipifera, Quercus, and Carya. Two additional equations encompassed the species included in the Jenkins et al. 2003 mixed hardwood (MH) and Hard maple/Oak/Hickory/Beech (MO) equations using a high number of TLS models. The additional equations were used for any individuals that were beyond the more specific equations diameter range in order to reduce bias in larger trees.

The grouping used in this study was based on the evaluation of multiple equation forms (data not shown). The diameter-based equation selected had the lowest AIC and highest AIC weight of all model forms tested. The inclusion of height reduced RMSE across all equations, but height was not measured across the site and thus not used in our analysis. Initially, individual species equations were evalu- 
Table 4.2: General allometry including the 10 species modeled with TLS. Equations were created for Liriodendron tulipifera $(\mathbf{L t})$, Quercus $(\mathbf{Q})$, and Carya $(\mathbf{C})$. The mixed hardwood equation (MH) includes Fraxinus americana, Liriodendron tulipifera, and Nyssa sylvatica. The Hard maple/Oak/Hickory/Beech equation (MO) includes Quercus, Carya, and Fagus grandifolia. RMSE is in log units and based on 5 -fold cross validation.

\begin{tabular}{llccccccc}
\hline \hline Eq. & Range $(\mathrm{cm})$ & $\mathrm{n}$ & $\beta_{0}$ & $\beta_{0}[\mathrm{se}]$ & $\beta_{1}$ & $\beta_{1}[\mathrm{se}]$ & $r^{2}$ & RMSE $(\mathrm{CV})$ \\
\hline Lt & $13-120$ & 47 & -1.9136 & 0.17 & 2.3513 & 0.04 & 0.98 & 0.16 \\
Q & $11-93$ & 66 & -1.5091 & 0.25 & 2.3237 & 0.07 & 0.95 & 0.24 \\
C & $10-47$ & 86 & -2.2249 & 0.25 & 2.5765 & 0.08 & 0.92 & 0.31 \\
MH & $11-120$ & 77 & -2.2647 & 0.16 & 2.4503 & 0.05 & 0.97 & 0.26 \\
MO & $10-105$ & 166 & -1.6637 & 0.14 & 2.3787 & 0.04 & 0.95 & 0.29 \\
\hline
\end{tabular}

ated, but no significant differences in slopes were found across multiple species. All Carya species were statistically similar. Only Quercus velutina was significantly different in the Quercus equation grouping. The Liriodendron tulipifera equation was prioritized because of the presence of this species across the site.

The Liriodendron tulipifera equation had the lowest uncertainty (RMSE $=$ $0.16)$ of all TLS allometry and the lowest equation sample size $(\mathrm{n}=47$; Table 2). The Carya equation had the highest uncertainty (RMSE $=0.31$ ) but the highest sample size for a single species equation $(\mathrm{n}=86)$. The MO equation had a high diameter range $(10-105 \mathrm{~cm})$ and the highest sample size $(\mathrm{n}=166)$. The MH equation had the highest diameter range $(11-120 \mathrm{~cm})$ of all equations and the slope coefficient $\left(\beta_{1}\right)$ was similar to the Jenkins et al. MH equation (2.4503 vs 2.4835, respectively), but RMSE was significantly lower (0.26 vs. 0.36 , respectively).

\subsubsection{Biomass Estimation and Uncertainty Analysis}

Tree biomass estimations from TLS models with TLS allometry, TLS allometry, and Jenkins et al. allometry were aggregated to the plot level to be used for the LiDAR empirical model. A total of 1,818 trees were included on the TLS plots (blue, Figure 4.1), but including the additional 11 plots for LiDAR calibration increased the sample to 3,947 trees. TLS models had the highest biomass estimates 

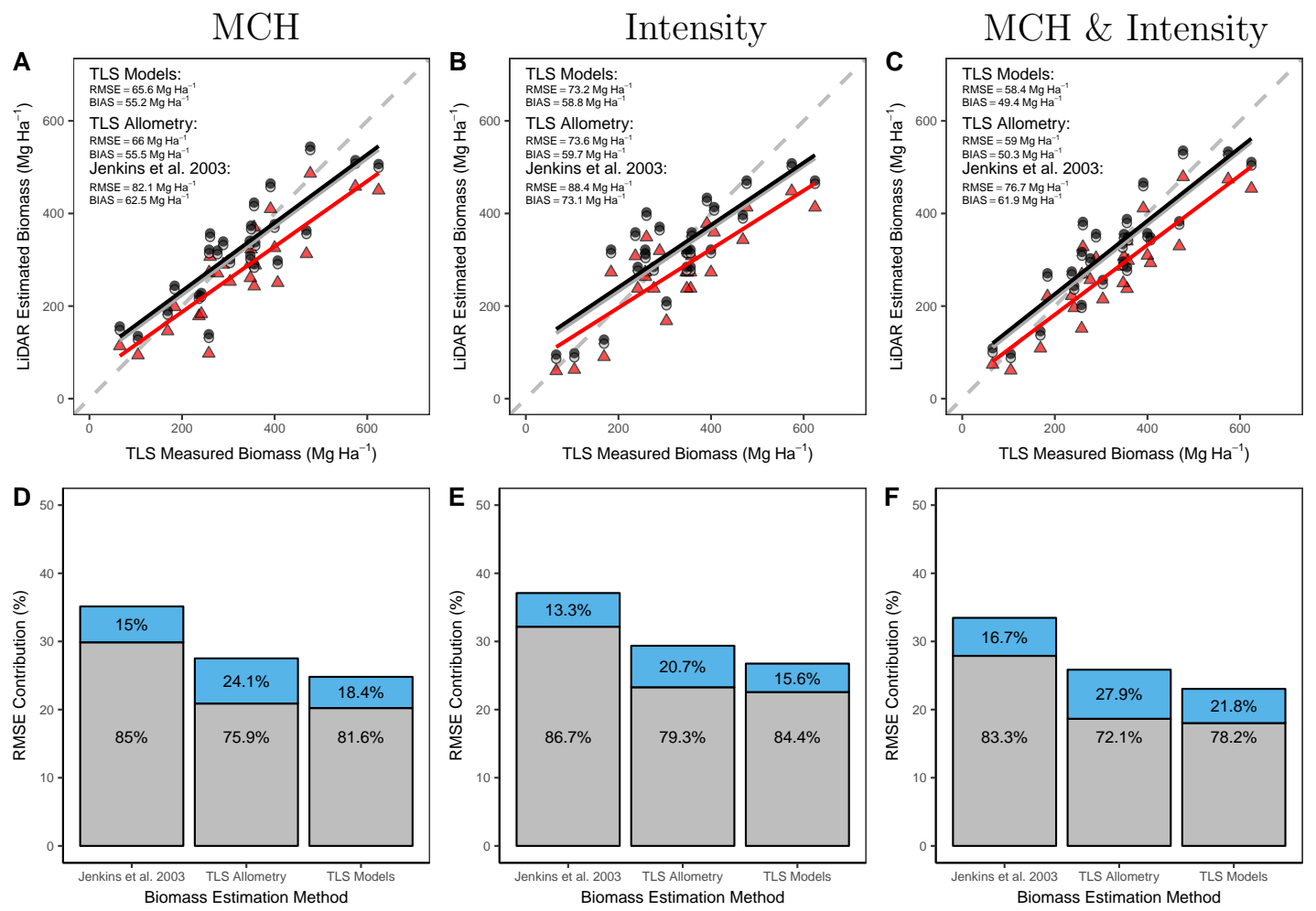

Figure 4.3: Comparison of LiDAR biomass calibration models (top row) and propagated uncertainty of biomass estimates (bottom row). Mean canopy height was used as a comparable canopy height metric collected using spaceborne LiDAR (GEDI or ICE-Sat II) (A \& D). LiDAR intensity was used as a comparable metric to radar backscatter, as collected using spaceborne PolSAR (B \& E). MCH and intensity were combined in the final model to emulate PolInSAR or TomoSAR (BIOMASS or NISAR) and potential cross-sensor fusion (C \& F). The TLS models (black) provided the lowest uncertainty estimates, followed by TLS allometry (grey), and Jenkins et al. 2003 allometry (red). TLS reduced the total propagated uncertainty of LiDAR calibration by over $10 \%$ (over $30 \%$ relative), with improvements in both plot-level and LiDAR calibration RMSE. Percentages within stacked bars indicate the relative contribution of uncertainty from allometry (blue) versus the LiDAR-derived empirical model (grey).

on average $\left(324 \pm 131 \mathrm{Mg} \mathrm{ha}^{-1}\right)$, followed by TLS allometry $\left(316 \pm 129 \mathrm{Mg} \mathrm{ha}^{-1}\right)$, and Jenkins et al. allometry $\left(275 \pm 126 \mathrm{Mg} \mathrm{ha}^{-1}\right)$. Relative plot uncertainty was lowest with TLS models (14.4\%), followed by TLS allometry (17.9\%), and Jenkins et al. allometry $(18.5 \%)$.

For the empirically derived LiDAR models, mean canopy height outperformed 
Table 4.3: Uncertainty of nine empirical biomass models based on calibration data from TLS models, TLS allometry, and Jenkins et al. 2003 equations. Three models are compared using mean canopy height $(\mathrm{MCH})$ and LiDAR intensity, as well as a multiple regression using both metrics as predictors. Uncertainty from model residuals $\left(\sigma_{\epsilon, \text { plot }}\right)$ and propagated uncertainty $\left(\sigma_{\epsilon, \text { pred }}\right)$ are reported in $\mathrm{Mg} \mathrm{ha}^{-1}$ and as a percentage of the mean (equivalent to relative RMSE).

\begin{tabular}{llcccc}
\hline \hline Model & Method & $\sigma_{\epsilon, \text { plot }}$ & $\sigma_{\epsilon, \text { plot }}(\%)$ & $\sigma_{\text {pred }}$ & $\sigma_{\text {pred }}(\%)$ \\
\hline$\beta_{0}+\beta_{1}(M C H)$ & TLS Models & 65.6 & 20.2 & 80.4 & 24.8 \\
& TLS Allometry & 66.0 & 20.9 & 87.0 & 27.5 \\
& Jenkins & 82.1 & 29.9 & 96.6 & 35.1 \\
\hline$\beta_{0}+\beta_{1}(I)$ & TLS Models & 73.2 & 22.6 & 86.7 & 26.7 \\
& TLS Allometry & 73.6 & 23.3 & 92.8 & 29.4 \\
& Jenkins & 88.4 & 32.2 & 102.0 & 37.1 \\
\hline$\beta_{0}+\beta_{1}(M C H)+\beta_{2}(I)$ & TLS Models & 58.4 & 18.0 & 74.7 & 23.0 \\
& TLS Allometry & 59.0 & 18.7 & 81.7 & 25.9 \\
& Jenkins & 76.7 & 27.9 & 92.0 & 33.5 \\
\hline
\end{tabular}

intensity as a predictor variable, and the combination offered the lowest uncertainty and bias (Table 4.3). Propagating allometric uncertainty to the LiDAR models increased model uncertainty in all cases. Use of TLS models over the Jenkins equations for LiDAR model calibration reduced model uncertainty through reduced plot-level uncertainty and lower residual error. For example, TLS models reduced the residual error by $9.7 \%$ and the propagated error by $10.3 \%$ in the $\mathrm{MCH}$ LiDAR model. TLS reduced uncertainty in all LiDAR models with the greatest reduction in the multiple regression model $(10.5 \%)$. The relative contribution of error from allometry increased with improved LiDAR models (Figure 4.3). The most uncertain model (Jenkins et al. equations with intensity) had the lowest relative contribution of uncertainty from allometry (13.3\%), while the least uncertain empirical model (TLS models with $\mathrm{MCH}$ and intensity) had a higher relative contribution to uncertainty from allometry. 


\subsection{Discussion}

The framework presented in this study uses TLS to reduce uncertainty at multiple levels of biomass estimation. We compared plot-level 3D stem reconstruction, TLS allometry, and the Jenkins et al. 2003 equations and the uncertainty associated with each approach. The five TLS-derived allometric equations created using 3D stem models had low uncertainty and high sample size, making them suitable for application across other plots within the site. Plot uncertainty was estimated from the RMSE in the allometric equations and propagated to the empirical plot biomass relationship from LiDAR metrics. The source of the majority of biomass estimation uncertainty was the residual error in the LiDAR model, but allometry contributed to $15-24.1 \%$ of the total uncertainty. A combined set of metrics using mean canopy height and return intensity produced the best model with low uncertainty. In all cases, TLS reduced uncertainty in biomass estimation.

\subsubsection{TLS for Local Non-destructive Allometry}

The findings from this study suggest TLS is an efficient means of creating nondestructive local allometry for estimating biomass. We scanned over the span of four days, averaging three $1 / 10^{\text {th }}$ ha plots per day. The relatively short time expenditure for field work resulted in 329 3D tree models (only 242 were used in this study). Other work has emphasized the cost and labor saved by using TLS in place of destructive sampling and we see similar benefits in this approach (Stovall et al., 2017).

Another clear benefit from TLS was the ability to estimate the biomass of several large trees above $70 \mathrm{~cm}$ that would otherwise be too costly or simply unfeasible to sample. The largest trees included in the national equations were $73 \mathrm{~cm}$, as opposed to $120 \mathrm{~cm}$ in our TLS allometry. Across all 3D modeled trees, TLS allometry estimated trees greater than $70 \mathrm{~cm}$ to have an average of $769 \mathrm{~kg}$ more biomass per tree than the Jenkins et al. equation estimates. The larger trees included in our allometry constrain estimates of biomass, thus reducing uncertainty in the upper diameter range. Moreover, direct modeling of large trees reduced plot uncertainty substantially, as these individuals have the highest potential for 
error. Further reductions in error were found when using height as an additional predictor variable, but inclusion of this variable was considered outside the scope of this study, as our primary interest was direct comparison to national allometry.

TLS allometry outperformed the Jenkins et al. 2003 equations in terms of RMSE. Given the scale of the Jenkins allometry, we expected this finding, as these equations encompass a much broader range of growing conditions than are found at our single site. As such, one of the major benefits and likely sources of error reduction in TLS allometry was the ability to create high sample size equations on a small spatial scale, limiting the impact of environmental variation in the equation. We decided to group species based on a preliminary allometric analysis by creating two genus-grouped equations with species as a categorical variable. We found species within the genus of Quercus and Carya to be statistically indistinguishable. The grouping of these individuals allowed us to increase equation sample size and reduce the number of species-specific equations needed - ultimately leading to a more reliable single equation. We did not include any assumptions about uncertainty in TLS models that would propagate to allometric equation error, but other work has shown QSM's to vary in RMSE from 5-15\% (Calders et al., 2015; Hackenberg et al., 2015). In the future we intend on quantifying this source of error and propagating it to higher levels to determine any impacts on biomass uncertainty. However, a similar, but more expansive uncertainty analysis elucidated small measurement errors in tree attributes at lower levels do not have a major impact on error when scaled to the landscape (Chen et al., 2015).

While TLS provided low uncertainty allometry in this study, there are still currently major limitations to the technology that impede widespread deployment. We collected the TLS data in low wind leaf-off conditions in order to clearly model the woody portion of the tree stem, but this scenario can only be achieved in certain areas. Deciduous forest ecosystems have the potential to benefit greatly from TLS, while dense tropical forests present significant challenges. A dense understory in any forest will substantially reduce visibility and increase occlusion. Standardized systematic sampling schemes have been suggested (Wilkes et al., 2017), but a thorough analysis of occlusion in the context of QSM's has yet to be completed. Rain and wind are also detrimental to the quality of TLS data and must be avoided if reliable volume estimates are required. However, the litany of potential prob- 
lems can often be overcome with appropriate planning and adjustments to field acquisition strategies (e.g. increasing the number of scans in a plot).

Total plot biomass was higher and uncertainty was markedly reduced at the plot level in both TLS scenarios compared to the Jenkins et al. 2003 equations. Given the reduction in uncertainty across allometric equations, we anticipated lower error at the plot level, but plot uncertainty only reduced by $4.1 \%$ with TLS models and $0.6 \%$ with TLS allometry. However, TLS model-based plot biomass was $49 \mathrm{Mg}$ $\mathrm{ha}^{-1}$ higher on average than the Jenkins equations, suggesting the improvements to biomass uncertainty may be realized at the calibration and validation stage through higher accuracy plot-level biomass values rather than increased precision. The higher estimates of biomass density using TLS suggests (1) this approach is sensitive to higher biomass in larger trees and (2) national-scale allometry may be underestimating the biomass of large trees in analogous forest types. Direct 3D modeling captures biomass density more accurately because biomass is not estimated with allometry. Since allometry estimates the mean value of biomass for a specific diameter extreme, examples of high biomass are unlikely to be estimated accurately. Allometry has been shown to systematically under- or overestimate biomass, depending on forest type (Duncanson et al., 2015a), highlighting the need for targeted TLS acquisitions for improved allometry or to directly estimate large tree biomass.

\subsubsection{Reducing Uncertainty in LiDAR Models}

Uncertainty is rarely propagated in analyses of biomass estimation with remote sensing methods (Chen et al., 2015) and no studies to our knowledge have quantified reductions to LiDAR biomass model uncertainty using TLS. Our approach uses TLS to reduce uncertainty from allometry and LiDAR calibration error. LiDAR calibration error is impacted primarily by the representative nature of the metric used to predict biomass ( $\mathrm{MCH}$ or intensity, in this study) and the accuracy of the field biomass estimates. We emphasize that nearly every remote sensing calibration and validation method for biomass estimation relies on estimates derived from empirical models rather than known values, except rarely, when destructive sampling of trees are used after data acquisition; in essence, an allometric model 
is used to calibrate metrics for a remote sensing model. TLS potentially provides a means to reduce the reliance on generalized allometric models for the calibration stage, thus reducing uncertainty. Since plot-level biomass can be estimated using a range of techniques, the chosen method can potentially have a dramatic impact on the final sensor calibration (Zhao et al., 2012). By quantifying improvements in empirical model calibration and propagating uncertainty we can make more informed decisions concerning sensor calibration plot data moving forward in estimating global forest biomass.

While there is disagreement in the literature over the appropriate plot size for biomass estimation, temperate plots are typically smaller (less than 1 ha) (Duncanson et al., 2015b) and tropical plots are larger (approximately 1 ha) (Chave et al., 2004). Larger plot sizes have been proposed in both systems as being superior for remote sensing calibration (Frazer et al., 2011), but we saw strong agreement with relatively small plots. TLS can reduce geolocation error when paired with airborne LiDAR, mitigating some of the uncertainty in the calibration stage (Hauglin et al., 2014). Moreover, ensuring tree crowns are not clipped on the plot edges, but rather included in the calculation of LiDAR metrics will improve residual error in the empirical model (Mascaro et al., 2011). Further investigations of plot size, geolocation errors, and edge effects are necessary to determine the role of TLS in reducing uncertainty from these sources and improving biomass model calibration.

Canopy height is almost certainly the most ubiquitous metric used to estimate biomass across an extensive range of forested ecosystems (Asner, 2009; Saatchi et al., 2011; Baccini et al., 2012; Fatoyinbo \& Simard, 2013; McRoberts et al., 2016). We used mean canopy height as an analogous metric to many of the upcoming satellite missions for estimating biomass. While our methods are directly applicable to the GEDI and Ice-SAT II missions because of the similar technology, the same findings apply to multi-sensor synthetic aperture radar configurations used to estimate forest height. The strong relationship between height and biomass found in this study confirms the findings of the overwhelming body of publications on the subject. TLS provided the most accurate and precise estimates of plot biomass and thus reduced the residual error in the LiDAR empirical model. The Jenkins et al. allometry systematically underestimated biomass den- 
sity and was less sensitive to high biomass plots. The strong agreement between TLS plot biomass estimates and LiDAR-derived mean canopy height is explained by TLS plot estimates being derived from the direct sensing of standing biomass rather than the indirect Jenkins et al. allometry. The strength of the LiDAR relationships were not anticipated given the low point cloud density (approximately 1 point $\mathrm{m}^{-2}$ ) of the airborne LiDAR acquisition, emphasizing the explanatory power of $\mathrm{MCH}$.

LiDAR intensity was nearly as successful as $\mathrm{MCH}$ for predicting plot biomass $\left(\mathrm{R}^{2}=64-70 \%\right)$, but the combination of the two variables provides the best model $\left(\mathrm{R}^{2}=80 \%\right)$. Past work has shown the utility of LiDAR intensity metrics for predicting forest biophysical parameters (Hudak et al., 2006; Zhao et al., 2012). The similarity of intensity to radar backscatter in the context of the current study's goals underscores the potential for TLS to reduce uncertainty in SAR biomass estimates for future satellite missions. The greatest benefits are realized with the combination of height and intensity metrics. In the combined model MCH contributed $43.7 \%$ of the model variability, versus the $37.7 \%$ explained by LiDAR intensity. The high contribution of intensity to predicting biomass in the combined model underscores not only the necessity for fusion of multiple metrics from individual sensors, but fusion of multiple sensors for improving biomass predictive capability on a global scale.

\subsection{Conclusion}

TLS is an effective and efficient means of reducing uncertainty in calibration of remote sensing missions estimating biomass. We determined TLS can reduce uncertainty in the future by:

(1) Providing a direct estimate of standing biomass by sensing woody volume, thus reducing the reliance on potentially unrepresentative allometry.

(2) Allowing the development of more representative non-destructive local allometric equations that can be applied to currently available plot data.

(3) Reducing residual variability in the LiDAR empirical modeling stage. 
TLS is unlikely to replace manual forest inventory completely, but adaptive methods may significantly improve plot-level biomass estimates for calibration and validation. Certain ecosystems like dense tropical forest present challenges for automated TLS inventory because occlusion reduces confidence in relatively simple measurements, but high quality single-tree biomass estimation in these systems can supplement limited allometry. Ultimately, the greatest future improvements to biomass mapping will be found with targeted TLS acquisitions in areas with non-existent local allometric equations and high biomass density. 


\section{References}

Asner, Gregory P. 2009. Tropical forest carbon assessment: integrating satellite and airborne mapping approaches. Environmental Research Letters, 4(3), 034009 .

Baccini, A., Goetz, SJ, Walker, WS, Laporte, NT, Sun, M, Sulla-Menashe, D, Hackler, J, Beck, PSA, Dubayah, R, Friedl, MA, \& others. 2012. Estimated carbon dioxide emissions from tropical deforestation improved by carbon-density maps. Nature Climate Change, 2(3), 182-185.

Bourg, Norman A., McShea, William J., Thompson, Jonathan R., McGarvey, Jennifer C., \& Shen, Xiaoli. 2013. Initial census, woody seedling, seed rain, and stand structure data for the SCBI SIGEO Large Forest Dynamics Plot: Ecological Archives E094-195. Ecology, 94(9), 2111-2112.

Calders, Kim, Newnham, Glenn, Burt, Andrew, Murphy, Simon, Raumonen, Pasi, Herold, Martin, Culvenor, Darius, Avitabile, Valerio, Disney, Mathias, Armston, John, \& Kaasalainen, Mikko. 2015. Nondestructive estimates of above-ground biomass using terrestrial laser scanning. Methods in Ecology and Evolution, 6(2), $198-208$.

Chave, Jerome, Condit, Richard, Aguilar, Salomon, Hernandez, Andres, Lao, Suzanne, \& Perez, Rolando. 2004. Error propagation and scaling for tropical forest biomass estimates. Philosophical Transactions of the Royal Society B: Biological Sciences, 359(1443), 409-420.

Chave, Jrme, Rjou-Mchain, Maxime, Brquez, Alberto, Chidumayo, Emmanuel, Colgan, Matthew S, Delitti, Welington BC, Duque, Alvaro, Eid, Tron, Fearnside, Philip M, Goodman, Rosa C, \& others. 2014. Improved allometric models to estimate the aboveground biomass of tropical trees. Global change biology, 20(10), 3177-3190.

Chen, Qi, Vaglio Laurin, Gaia, \& Valentini, Riccardo. 2015. Uncertainty of remotely sensed aboveground biomass over an African tropical forest: Propagating errors from trees to plots to pixels. Remote Sensing of Environment, 160(Apr.), 134-143. 
Chojnacky, D. C., Heath, L. S., \& Jenkins, Jennifer C. 2014. Updated generalized biomass equations for North American tree species. Forestry, 87(1), 129-151.

Duncanson, L., Rourke, O., \& Dubayah, R. 2015a. Small Sample Sizes Yield Biased Allometric Equations in Temperate Forests. Scientific Reports, 5(Nov.), 17153.

Duncanson, L. I., Dubayah, R. O., Cook, B. D., Rosette, J., \& Parker, G. 2015b. The importance of spatial detail: Assessing the utility of individual crown information and scaling approaches for lidar-based biomass density estimation. Remote Sensing of Environment, 168, 102 - 112.

Duncanson, Laura I., Dubayah, Ralph O., \& Enquist, Brian J. 2015c. Assessing the general patterns of forest structure: quantifying tree and forest allometric scaling relationships in the United States: Forest allometric variability in the United States. Global Ecology and Biogeography, 24(12), 1465-1475.

Fatoyinbo, Temilola E., \& Simard, Marc. 2013. Height and biomass of mangroves in Africa from ICESat/GLAS and SRTM. International Journal of Remote Sensing, 34(2), 668-681.

Frazer, G.W., Magnussen, S., Wulder, M.A., \& Niemann, K.O. 2011. Simulated impact of sample plot size and co-registration error on the accuracy and uncertainty of LiDAR-derived estimates of forest stand biomass. Remote Sensing of Environment, 115(2), 636-649.

Goetz, Scott, \& Dubayah, Ralph. 2011. Advances in remote sensing technology and implications for measuring and monitoring forest carbon stocks and change. Carbon Management, 2(3), 231-244.

Hackenberg, Jan, Wassenberg, Marc, Spiecker, Heinrich, \& Sun, Dongjing. 2015. Non destructive method for biomass prediction combining TLS derived tree volume and wood density. Forests, 6(4), 1274-1300.

Hall, Forrest G., Bergen, Kathleen, Blair, James B., Dubayah, Ralph, Houghton, Richard, Hurtt, George, Kellndorfer, Josef, Lefsky, Michael, Ranson, Jon, Saatchi, Sasan, Shugart, H. H., \& Wickland, Diane. 2011. Characterizing 3D vegetation structure from space: Mission requirements. Remote Sensing of Environment, 115(11), 2753 - 2775 .

Hauglin, Marius, Lien, Vegard, Nsset, Erik, \& Gobakken, Terje. 2014. Georeferencing forest field plots by co-registration of terrestrial and airborne laser scanning data. International Journal of Remote Sensing, 35(9), 3135-3149. 
Houghton, R. A., Hall, Forrest, \& Goetz, Scott J. 2009. Importance of biomass in the global carbon cycle. Journal of Geophysical Research-Biogeosciences, 114(Sept.), G00E03.

Hudak, Andrew T., Crookston, Nicholas L., Evans, Jeffrey S., Falkowski, Michael J., Smith, Alistair MS, Gessler, Paul E., \& Morgan, Penelope. 2006. Regression modeling and mapping of coniferous forest basal area and tree density from discrete-return lidar and multispectral satellite data. Canadian Journal of Remote Sensing, 32(2), 126-138.

Jenkins, J. C., Chojnacky, David C., Heath, Linda S., \& Birdsey, Richard A. 2003. National-scale biomass estimators for United States tree species. Forest Science, 49(1), 12-35.

Mascaro, Joseph, Detto, Matteo, Asner, Gregory P., \& Muller-Landau, Helene C. 2011. Evaluating uncertainty in mapping forest carbon with airborne LiDAR. Remote Sensing of Environment, 115(12), 3770-3774.

McRoberts, Ronald E., Chen, Qi, Domke, Grant M., Sthl, Gran, Saarela, Svetlana, \& Westfall, James A. 2016. Hybrid estimators for mean aboveground carbon per unit area. Forest Ecology and Management, 378(Oct.), 44-56.

Mitchard, Edward T. A., Feldpausch, Ted R., Brienen, Roel J. W., LopezGonzalez, Gabriela, Monteagudo, Abel, Baker, Timothy R., Lewis, Simon L., Lloyd, Jon, Quesada, Carlos A., Gloor, Manuel, ter Steege, Hans, Meir, Patrick, Alvarez, Esteban, Araujo-Murakami, Alejandro, Aragao, Luiz E. O. C., Arroyo, Luzmila, Aymard, Gerardo, Banki, Olaf, Bonal, Damien, Brown, Sandra, Brown, Foster I., Ceron, Carlos E., Chama Moscoso, Victor, Chave, Jerome, Comiskey, James A., Cornejo, Fernando, Corrales Medina, Massiel, Da Costa, Lola, Costa, Flavia R. C., Di Fiore, Anthony, Domingues, Tomas F., Erwin, Terry L., Frederickson, Todd, Higuchi, Niro, Honorio Coronado, Euridice N., Killeen, Tim J., Laurance, William F., Levis, Carolina, Magnusson, William E., Marimon, Beatriz S., Marimon Junior, Ben Hur, Mendoza Polo, Irina, Mishra, Piyush, Nascimento, Marcelo T., Neill, David, Nunez Vargas, Mario P., Palacios, Walter A., Parada, Alexander, Pardo Molina, Guido, Pena-Claros, Marielos, Pitman, Nigel, Peres, Carlos A., Poorter, Lourens, Prieto, Adriana, RamirezAngulo, Hirma, Restrepo Correa, Zorayda, Roopsind, Anand, Roucoux, Katherine H., Rudas, Agustin, Salomao, Rafael P., Schietti, Juliana, Silveira, Marcos, de Souza, Priscila F., Steininger, Marc K., Stropp, Juliana, Terborgh, John, Thomas, Raquel, Toledo, Marisol, Torres-Lezama, Armando, van Andel, Tinde R., van der Heijden, Geertje M. F., Vieira, Ima C. G., Vieira, Simone, Vilanova-Torre, Emilio, Vos, Vincent A., Wang, Ophelia, Zartman, Charles E., 
Malhi, Yadvinder, \& Phillips, Oliver L. 2014. Markedly divergent estimates of Amazon forest carbon density from ground plots and satellites. Global Ecology and Biogeography, 23(8), 935-946.

Othmani, Ahlem, Piboule, Alexandre, Krebs, M., Stolz, C., \& Voon, LFC Lew Yan. 2011. Towards automated and operational forest inventories with T-Lidar. In: 11th International Conference on LiDAR Applications for Assessing Forest Ecosystems (SilviLaser 2011).

Picard, Nicolas, Saint-Andre, Laurent, \& Henry, Matieu. 2012. Manual for building tree volume and biomass allometric equations: from field measurement to prediction. Food and Agricultural Organization of the United Nations and Centre de Coopration Internationale en Recherche Agronomique pour le Dveloppement.

Raumonen, Pasi, Kaasalainen, Mikko, kerblom, Markku, Kaasalainen, Sanna, Kaartinen, Harri, Vastaranta, Mikko, Holopainen, Markus, Disney, Mathias, \& Lewis, Philip. 2013. Fast Automatic Precision Tree Models from Terrestrial Laser Scanner Data. Remote Sensing, 5(2), 491-520.

Saatchi, Sassan, Harris, Nancy L., Brown, Sandra, Lefsky, Michael, Mitchard, Edward T. A., Salas, William, Zutta, Brian R., Buermann, Wolfgang, Lewis, Simon L., Hagen, Stephen, Petrova, Silvia, White, Lee, Silman, Miles, \& Morel, Alexandra. 2011. Benchmark map of forest carbon stocks in tropical regions across three continents. Proceedings of the National Academy of Sciences of the United States of America, 108(24), 9899-9904.

SCENE (version 5.4.4.41689). 2015. FARO Technologies Inc.

Stovall, Atticus E.L., Vorster, Anthony G., Anderson, Ryan S., Evangelista, Paul H., \& Shugart, Herman H. 2017. Non-destructive aboveground biomass estimation of coniferous trees using terrestrial LiDAR. Remote Sensing of Environment, 200(Oct.), 31-42.

Wagner, Wolfgang, Ullrich, Andreas, Ducic, Vesna, Melzer, Thomas, \& Studnicka, Nick. 2006. Gaussian decomposition and calibration of a novel small-footprint full-waveform digitising airborne laser scanner. ISPRS Journal of Photogrammetry and Remote Sensing, 60(2), 100-112.

West, Geoffrey B., Brown, James H., \& Enquist, Brian J. 1999. A general model for the structure and allometry of plant vascular systems. Nature, 400(6745), 664-667. 
Wickland, D., Gobron, N., Moore, B., Nakajima, M., Sathyendranath, S., Plummer, S., Schmullius, C., \& Dubayah, R. 2014. CEOS Strategy for Carbon Observations from Space. In: 40th COSPAR Scientific Assembly. COSPAR Meeting, vol. 40 .

Wilkes, Phil, Lau, Alvaro, Disney, Mathias, Calders, Kim, Burt, Andrew, Gonzalez de Tanago, Jose, Bartholomeus, Harm, Brede, Benjamin, \& Herold, Martin. 2017. Data acquisition considerations for Terrestrial Laser Scanning of forest plots. Remote Sensing of Environment, 196(July), 140-153.

Zhao, Feng, Guo, Qinghua, \& Kelly, Maggi. 2012. Allometric equation choice impacts lidar-based forest biomass estimates: A case study from the Sierra National Forest, CA. Agricultural and Forest Meteorology, 165(Nov.), 64-72. 


\section{Chapter 5}

Moving Forward with Terrestrial LiDAR

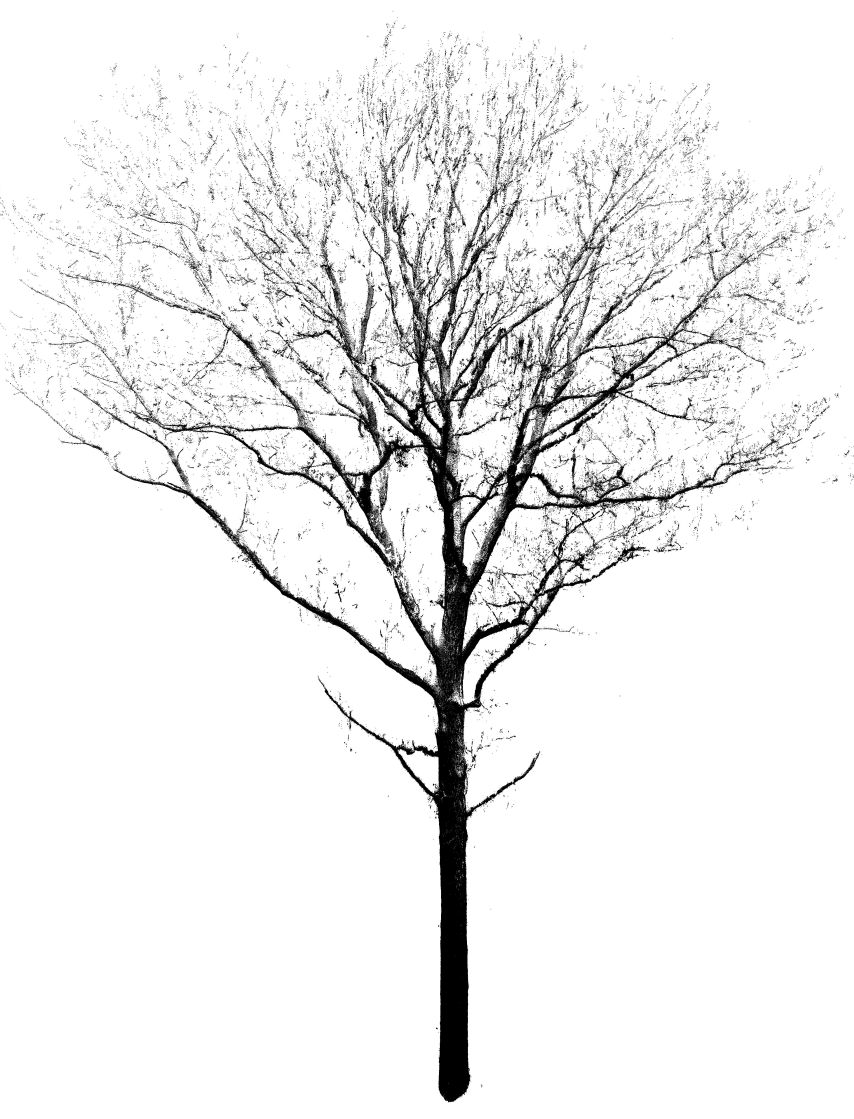




\section{Reducing Biomass Uncertainty with TLS and Next Steps}

The investigations presented in this work quantified the potential for terrestrial LiDAR to improve estimates of biomass and reduce uncertainty at multiple spatial scales. We asked three primary questions, aimed at assessing TLS for estimating non-destructive tree-level biomass, developing non-destructive allometry, and calibration of airborne and spaceborne sensors for landscape or global biomass estimation. The questions will briefly be discussed in context with the research presented, followed by the immediate next steps in each research area.

[1] Can terrestrial LiDAR be used to estimate biomass at the individual tree scale?

Terrestrial LiDAR is an effective tool for estimating individual tree biomass, but many of the current approaches only consider whole-tree biomass (Calders et al., 2015) and are not accurate with noisy point clouds (Hackenberg et al., 2015). We developed an algorithm that not only accurately estimated whole tree biomass, but was capable of estimating the contribution from individual components (e.g. trunk, branch, and foliage). The OHM algorithm utilized a novel convex hull peeling approach for trunk biomass estimation that was effective in the presence of high noise point clouds collected with lower-cost TLS technology. The convex hull peeling method was unique in that the exterior trunk structure was accurately recreated, rather than assuming a cylindrical shape. We quantified the uncertainty from applying measured versus average species-specific wood density using the OHM method and, while measured wood density reduced error, it was only reliable for the trunk component. The needle and branch algorithm relied on the low error trunk estimates to estimate inner branch diameter, facilitating modeling of heightspecific branch and needle distributions. The branch and needle algorithm had higher uncertainty than the trunk estimates, but, given the presence of substantial noise in the point cloud, provided relatively unbiased, spatially explicit estimates of these two components.

The OHM algorithm can be improved in the future through further testing. The highest initial priority in development will be automation of trunk segmentation. The processing framework currently implemented in the OHM algorithm will facilitate these improvements. We are testing automatic segmentation through 
convex hull peeling on unsegmented point clouds. Essentially, convex hull peeling will initiate at the outermost branch and peel away the exterior points, revealing the innermost trunk structure. At the very least, this approach would provide a rough estimate of the trunk area, reducing the time expenditure for manual segmentation. Additional species must me tested to determine how accuracy and precision depends on tree structures different from the 21 lodgepole pine samples. The destructive sampling data from Chapter 2 is part of a larger project with an additional 20 trees of ponderosa pine and douglas fir. The remaining destructive samples will be tested using the OHM algorithm to guide future developments. With further testing and automation the OMH method may become a viable alternative to cylinder fitting algorithms in noisy TLS data.

[2] How does locally derived terrestrial LiDAR allometry influence estimates of biomass compared to the more commonly used allometric relationships?

National allometry built from destructive samples across broad spatial areas has become the the standard for biomass estimation (Chave et al., 2005, 2014). Pooling of large datasets is necessary in order to increase the predictive power of relationships, but species-specific trends are masked, reducing sensitivity to spatial patterns at the mapping stage (Mitchard et al., 2014). Terrestrial laser scanning can accurately estimate single-tree biomass, but minimal work has focused on the potential for non-destructive allometric equation development (Olagoke et al., 2016; Momo Takoudjou et al., 2017). We modeled 329 trees across 14 plot locations and developed 30 species-specific allometric equations. The TLS allometry had lower uncertainty than the national equations, but a wider range of equation error. The tree models validated Jenkins et al. (2003) and Chojnacky et al. (2014) allometry, revealing a consistent underestimation in both equations. We evaluated the effect of sample size and large trees on predictive accuracy of allometry. Diameter allometry required approximately 180 samples before equation parameters were consistent, while the diameter-height equation only needed 100 samples. Large trees are essential for accurate biomass estimates, especially if diameter is the only predictive variable. Including height in allometry reduced the sensitivity to the removal of large trees, so height should be measured whenever possible. 
Expansion to other areas is necessary to determine the broad applicability of non-destructive allometric equation development. While the highest quality TLS models will be built in leaf-off conditions, many deciduous forests have been extensively studied and improvements to allometry may be minimal. Targeted TLS acquisitions in deciduous forests with limited species-specific allometry can immediately improve biomass estimates through non-destructive high sample size allometry. The greatest challenge remains in broadleaf evergreen or tropical forests. Substantial testing of TLS in these ecosystems is needed before 3D models are used for allometric equation development, primarily due to uncertainty due to occlusion from dense vegetation. Some systems with open understory may be good initial candidates for TLS deployment in tropical systems. In Gabon, the tallest mangrove forest on Earth reaches approximately $60 \mathrm{~m}$ and has an open understory with minimal mid-story vegetation. Similar systems may allow measurement of extremely large trees with relatively full coverage, increasing the number of samples in and spatial coverage of tropical allometry.

[3] Can terrestrial LiDAR directly reduce uncertainty in sensor calibration, validation, and biomass mapping through plot-level 3D modeling and non-destructive allometric equations?

Global biomass estimates are driven by uncertain tree-level allometric relationships (Saatchi et al., 2011; Baccini et al., 2012). With the rapid development of terrestrial LiDAR for non-destructive biomass estimates through three-dimensional modeling, uncertainty at the plot level can be reduced for improved sensor calibration. We quantified uncertainty reduction with TLS through three-dimensional reconstruction and non-destructive allometry compared to national-scale allometry. Terrestrial LiDAR reduces uncertainty at the plot level, but also at the calibration stage. We determined TLS can potentially reduce sensor calibration uncertainty by over $10 \%$. Allometric uncertainty is of greater relative importance when sensor calibration is more precise. TLS was also more sensitive to higher biomass plot locations, indicating potential in areas of high biomass and high uncertainty that are difficult to estimate with allometry. The framework outlined provides a methodology that can immediately be applied in similar forests for reduced uncertainty sensor calibration and biomass mapping. 


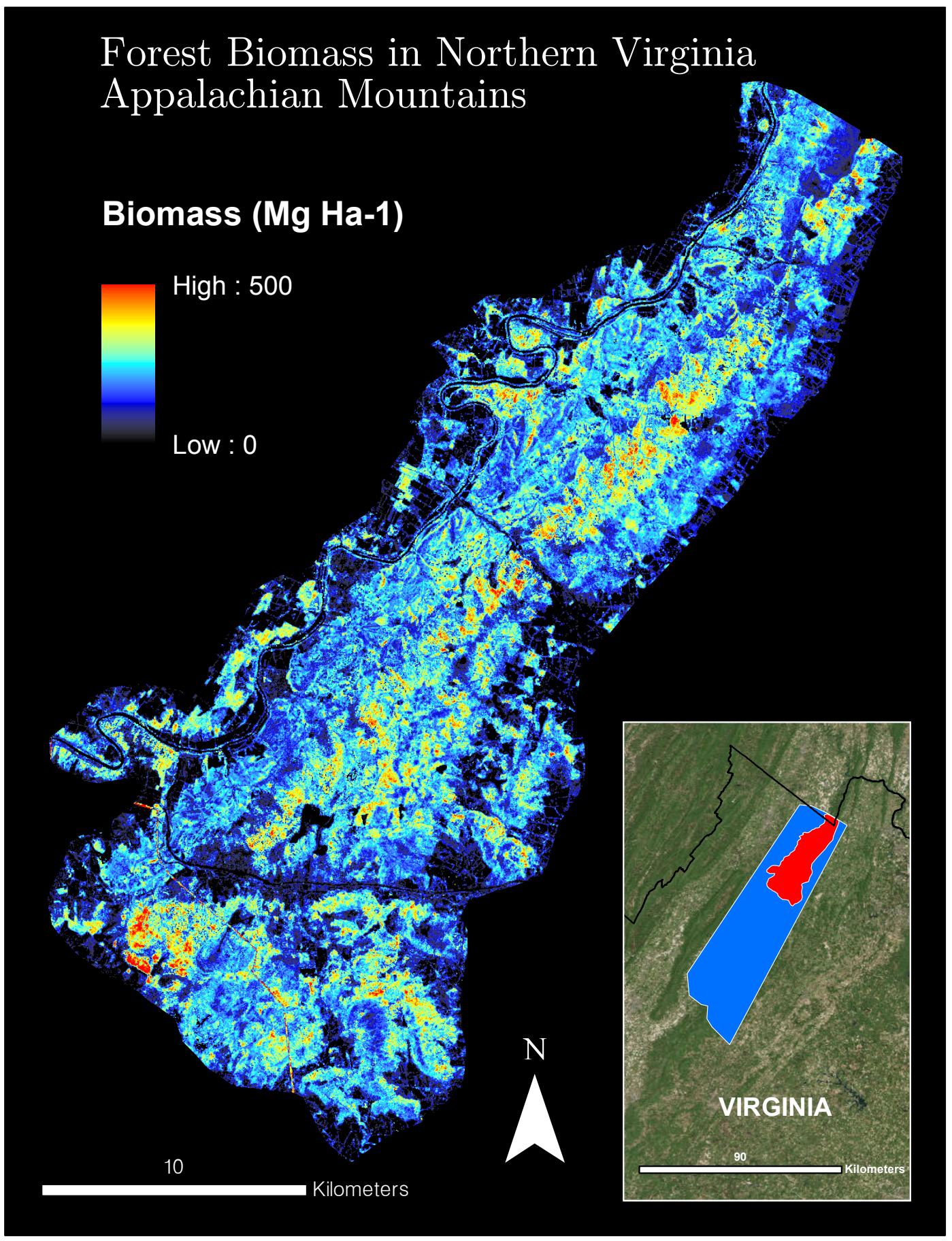

Figure 5.1: Area-wide LiDAR-derived biomass estimates, based on mean forest canopy height and TLS calibration. The processed area (red) is part of a larger acquisition in Virginia (blue). 
While we assessed TLS in the context of sensor calibration, variation in the spatial distribution of biomass under different plot-level data must be considered. The airborne LiDAR data used for the analysis in Chapter 4 is part of a much larger acquisition. Initial processing of the LiDAR from this 50,831 ha area reveals an average biomass density of $195 \mathrm{Mg} \mathrm{ha}^{-1}$ and over $50 \mathrm{Tg}$ of biomass, half of which is carbon (Figure 5.1). The landscape-level estimates are built from the 25 calibration plots at SCBI, but additional plot data will be necessary to improve confidence in this biomass map. FIA data is available throughout the LiDAR acquisition area, allowing improved calibration and expansion to other LiDAR datasets in Virginia. The baseline biomass distributions from the Jenkins et al. (2003) spatial distribution and estimates must be compared to the TLS derived map. We anticipate landscape-level differences in magnitude between the two maps, with Jenkins equations underestimating area wide biomass. The CMS United States biomass product (Hagen et al., 2016) estimates an average of 105 $\mathrm{Mg} \mathrm{ha}{ }^{-1}$ over the same area, suggesting a need for comparison of several estimates to TLS-derived values.

Another essential future goal in moving the proposed TLS framework forward is testing in new ecosystems. The allometric equations in the United States are more robust than many other locations and are thus likely to see the least relative improvement in uncertainty, unless improved species-specific regional equations are developed (McRoberts et al., 2016). Deployment of TLS in areas with stark disagreement or high uncertainty in LiDAR calibration models may see substantial improvements from improved allometry and/or direct three-dimensional reconstruction. The Pacific Northwest is estimated to have the highest aboveground biomass density of any forest on Earth and airborne LiDAR has been unable to capture this with height metrics (Duncanson et al., 2017). The uncertainty of plot estimates may be attributed to biased allometric models or lack of explanatory power of LiDAR height. Given our findings, we anticipate improvements to allometry in these high biomass density systems that may reduce LiDAR calibration error. 


\section{Moving Forward}

The clear finding from the work presented in this dissertation is that terrestrial LiDAR has a major role to play in reducing uncertainty in future forest measurement and monitoring. Beyond these initial investigations, the current challenges, potential for operational deployment, and future questions to be answered with TLS must be considered. True benefits from TLS will come when the technology becomes accessible, standardized, and consistent. Continued progress in TLS applications will provide necessary information that will inform the next generation of ecological questions.

\section{Current Challenges for Operational Deployment}

Much like early development of airborne LiDAR, TLS is on a path towards standardization and operational deployment. The body of literature and work presented in this dissertation has underscored the potential for TLS to be incorporated as a standard forestry measurement tool (Liang et al., 2016), but there are still major limitations and developments needed before TLS can be operationally deployed. One major hurdle in TLS is initial cost. The highest quality instruments, in terms of durability and scan quality, are cost prohibitive for most institutions, so they have primarily been reserved as research tools for experimental use. Commercial instrument costs have continued on a steady downward trend, but few new instruments have been tested in a forest context. If non-destructive biomass estimation is the goal, new, less expensive sensors will need to be tested with destructive harvesting data in order to ensure instrument dependent error and noise does not bias tree models. This is particularly relevant in the context of our work, as we sought to apply our methodology with a more portable, lower cost instrument, but did so with the trade-off of increased noise. As TLS technology becomes more affordable, low-noise instruments will be more attainable, but, until then, trade-offs in quality associated with entry cost is a major consideration for widespread adoption of TLS in forestry.

TLS is limited substantially by field conditions - many instruments are currently heavy and sensitive to temperature and moisture. Tropical forests are an example where both conditions are at an extreme. Recent developments have im- 
proved water resistance, but extremely high moisture environments are detrimental to scan quality, as TLS laser wavelengths often reside in the water absorption range of the electromagnetic spectrum. Tropical forests with high density understory cause occlusion and the only current solution is to increase scan positions (Wilkes et al., 2017), emphasizing the importance for quick instrument scan times, so acquisition does not become prohibitively time consuming. Standardization of acquisition strategies will be required for future interoperability, but the complexity of forest environments makes standardized sampling difficult to achieve. A consistent sampling approach, optimized for forest type, will be essential as TLS is more widely used and consistent data products are required. Mobile applications, as well as in situ monitoring of scan coverage, may provide a solution to the sampling problem, ensuring sufficient and consistent coverage. Improvements to portability, resistance to environmental conditions, and instrument technology will eventually allow deployment of TLS in most forest settings.

The wide array of algorithms used for non-destructive biomass estimation with TLS data will need to be synthesized and optimized according to forest type before the technology is broadly applicable. QSMs appear to be the most widely used of the volume estimation algorithms (Raumonen et al., 2013), but a range of other approaches, namely the OHM approach developed in Chapter 2, have been optimized to accurately perform in situations where QSMs fail. Other major issues, such as the influence of occlusion on automated volume estimates, must be addressed in simulation space, in order to optimize algorithms for several forest types. Once algorithms have been sufficiently tested in a range of real or simulated ecosystems, processing accessibility needs to be the highest priority. CompuTree is an excellent example of an early stage open source TLS processing application (Othmani et al., 2011). Consistent development and community involvement will be necessary for efficient optimization of many of the automated algorithms included in end user programs. As more options for processing become available, TLS will be easier to adopt and use in an operational setting, thus providing unprecedented and widespread capacity to address ecological and management questions relevant to forest ecosystems. 


\section{Future Questions and Applications}

Terrestrial LiDAR is currently the best method of reconstructing ecosystems with fine-scale three-dimensional detail, but the potential of this tool has yet to fully be realized in the context of ecosystem science. With focused research and development, TLS can help answer previously unattainable ecosystem-level questions.

Future measurement and monitoring of carbon stocks can be improved with terrestrial LiDAR. The work presented in this dissertation has shown the potential for widespread application of terrestrial LiDAR for non-destructive biomass estimation and allometric equation development, but as sensors become less expensive, this technology can potentially be regularly incorporated into forest inventory for reduced uncertainty carbon measurement and monitoring. With an increasing number of TLS models, allometry can be stratified according to environmental conditions (e.g. slope, aspect, elevation, solar radiation, soil moisture, nutrients, etc.), not only further improving biomass estimates, but also the understanding of those environmental factors most influencing biomass allocation. Unmanned stationary TLS sensors measuring vegetation structure have already been developed and tested for monitoring change over time (Culvenor et al., 2014), giving a glimpse of one potential avenue on this front. At the plot and stand scale, TLS can easily detect change in three dimensional structure (Srinivasan et al., 2014), quantifying movement from above- to below-ground carbon pools. The non-destructive nature of TLS modeling allows for longitudinal studies of tree growth, giving insight to the yearly changes in biomass and those factors influencing the rate of accumulation.

The capability of TLS as a tool for quantifying three-dimensional structure makes it ideal for characterizing wildlife habitat. TLS has been successfully used to characterize 3D space for monitoring bat activity (Yang et al., 2013). For bird species, subjective estimates of canopy complexity or simple measurements of cover are often used to assess habitat suitability. Airborne LiDAR has been used to map bird habitat successfully, but with high uncertainty (Goetz et al., 2010). The uncertainty in these mapping estimates may be due to inadequate characterization in the three-dimensional distribution of vegetation - an easily retrievable parameter with TLS. Moreover, TLS may provide additional structural information that can 


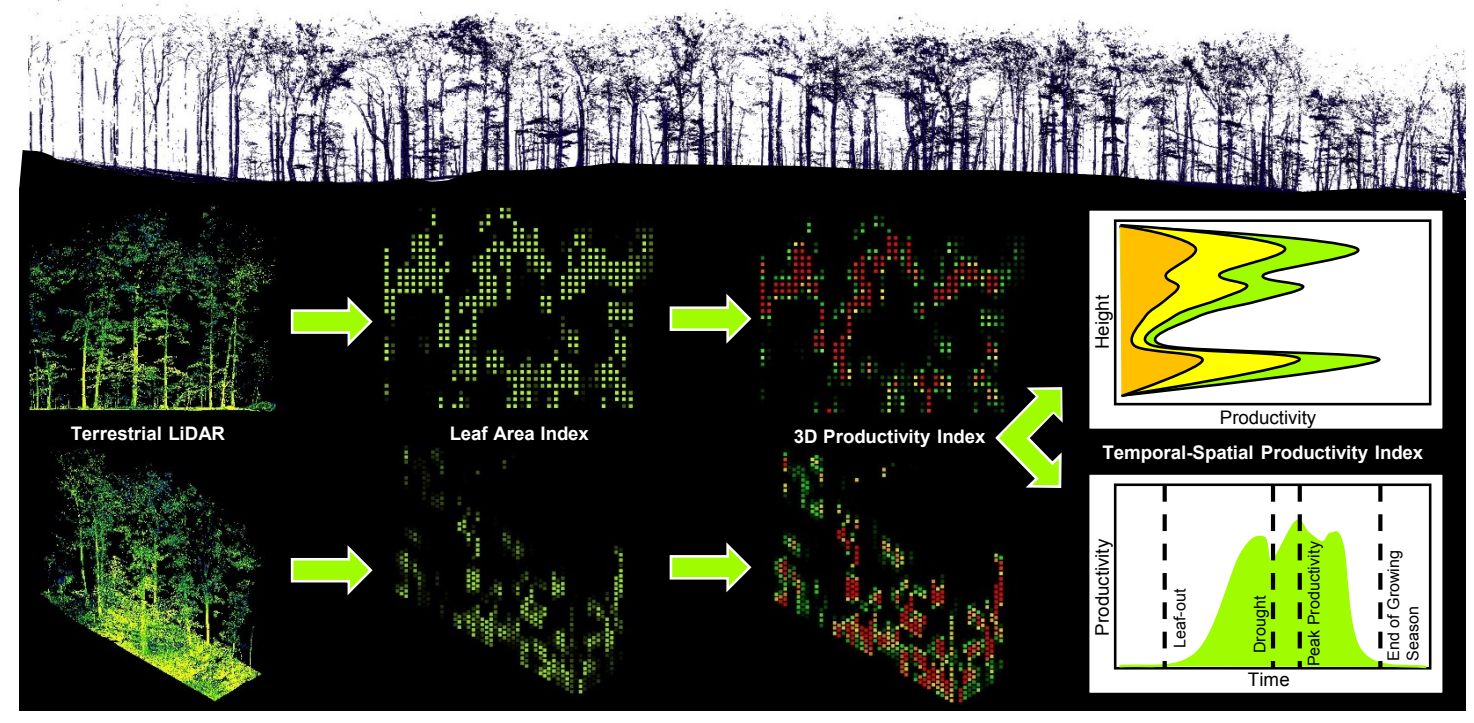

Figure 5.2: Potential TLS sensor fusion approach, allowing temporal and spatial monitoring of ecosystem status over large area acquisitions.

be used to validate airborne methods (Hancock et al., 2017) for improved habitat characterization and mapping. Incorporating TLS measurements of ecosystem structure as a tool for relatively objective wildlife habitat assessment has significant implications for management decisions if this tool can provide higher quality estimates of habitat range and quality.

The potential for sensor fusion with TLS is substantial. TLS can bridge the gap between leaf-level and ecosystem-level measurements. Canopy leaf area arrangement impacts ecosystem productivity and TLS is capable of providing high resolution three-dimensional leaf area distribution (Bland et al., 2014; Jin et al., 2016). Solar induced florescence can provide quality estimates of productivity (Yang et al., 2015). Together, the two measurements could help disentangle the effects of leaf distribution and leaf-level production efficiency. Over time, the fusion of structure and estimates of productivity could improve monitoring of forest health and uncover impacts of ecosystem stressors (Figure 5.2). Large TLS acquisitions over 1 ha are becoming more common (Calders et al., 2016) and are ideal for this type of analysis, providing fine scale vegetation distribution across the landscape. Coupled with flux tower measurements of $\mathrm{CO}_{2}$ exchange, TLS data fusion can begin to accurately quantify ecosystem structure-production relationships. 
Many of the findings from TLS studies can be directly applied to individualbased forest gap models (Shugart et al., 2015). Starting with parameterization, TLS can improve the vertical distribution of biomass and leaf area index - essential for modeling competitive interactions. Leaf area distributions within model frameworks are built from crown architecture assumptions, with little empirical data. TLS can validate model-derived leaf area distributions or provide parameterization information on 3D leaf area. Using space-for-time substitution, TLS can characterize, in detail, the three-dimensional structure of forests at different stages of succession (Cuni-Sanchez et al., 2016), producing more realistic model output. Moreover, as TLS models are created more readily, they can be incorporated into modeling environments for realistic plot representations or interactive competitive processes. Spatially explicit competitive interactions in real forests can be quantified using TLS through crown reconstruction and potentially used to inform forest models. The volume of detailed information on tree and stand structure available in TLS data is capable of significantly improving forest models in the future.

\section{Conclusion}

Terrestrial laser scanning is capable of enhancing vegetation measurements, impacting understanding and management of forest ecosystems at a global scale. The interconnected nature of up-scaling approaches with global remote sensing missions points to the need for unbiased plot-level measurements. TLS is capable of being immediately deployed as an operational tool for allometric equation development in needleleaf evergreen, broadleaf deciduous, and, with sufficient quality control, tropical forests. Substantial improvements to biomass allometry using TLS will become a staple of the benchmark carbon maps created using the upcoming GEDI, NISAR, and BIOMASS missions. With improved global carbon estimates, human impacts on forests, such as deforestation and degradation, can be quantified more accurately, improving national-scale carbon budgets. The framework presented in this work aims to address these primary issues. With targeted deployment in the coming years, TLS will reduce uncertainty in global biomass estimates, while providing unprecedented measures of three-dimensional structure that stand to reshape the capacity for complex ecosystem-level investigations. 


\section{References}

Baccini, A., Goetz, SJ, Walker, WS, Laporte, NT, Sun, M, Sulla-Menashe, D, Hackler, J, Beck, PSA, Dubayah, R, Friedl, MA, \& others. 2012. Estimated carbon dioxide emissions from tropical deforestation improved by carbon-density maps. Nature Climate Change, 2(3), 182-185.

Bland, Martin, Baldocchi, Dennis D., Widlowski, Jean-Luc, Fournier, Richard A., \& Verstraete, Michel M. 2014. On seeing the wood from the leaves and the role of voxel size in determining leaf area distribution of forests with terrestrial LiDAR. Agricultural and Forest Meteorology, 184(Jan.), 82-97.

Calders, Kim, Newnham, Glenn, Burt, Andrew, Murphy, Simon, Raumonen, Pasi, Herold, Martin, Culvenor, Darius, Avitabile, Valerio, Disney, Mathias, Armston, John, \& Kaasalainen, Mikko. 2015. Nondestructive estimates of above-ground biomass using terrestrial laser scanning. Methods in Ecology and Evolution, 6(2), $198-208$.

Calders, Kim, Burt, Andrew, Origo, Niall, Disney, Mathias, Nightingale, J, Raumonen, Pasi, \& Lewis, Philip. 2016. Large-area virtual forests from terrestrial laser scanning data. Pages 1765-1767 of: Geoscience and Remote Sensing Symposium (IGARSS), 2016 IEEE International. IEEE.

Chave, J., Andalo, C., Brown, S., Cairns, M. A., Chambers, J. Q., Eamus, D., Flster, H., Fromard, F., Higuchi, N., Kira, T., Lescure, J.-P., Nelson, B. W., Ogawa, H., Puig, H., Rira, B., \& Yamakura, T. 2005. Tree allometry and improved estimation of carbon stocks and balance in tropical forests. Oecologia, 145(1), 87-99.

Chave, Jrme, Rjou-Mchain, Maxime, Brquez, Alberto, Chidumayo, Emmanuel, Colgan, Matthew S, Delitti, Welington BC, Duque, Alvaro, Eid, Tron, Fearnside, Philip M, Goodman, Rosa C, \& others. 2014. Improved allometric models to estimate the aboveground biomass of tropical trees. Global change biology, 20(10), 3177-3190. 
Culvenor, Darius, Newnham, Glenn, Mellor, Andrew, Sims, Neil, \& Haywood, Andrew. 2014. Automated In-Situ Laser Scanner for Monitoring Forest Leaf Area Index. Sensors, 14(8), 14994-15008.

Cuni-Sanchez, Aida, White, Lee JT, Calders, Kim, Jeffery, Kathryn J., Abernethy, Katharine, Burt, Andrew, Disney, Mathias, Gilpin, Martin, Gomez-Dans, Jose L., \& Lewis, Simon L. 2016. African savanna-forest boundary dynamics: A 20-year study. PloS one, 11(6), e0156934.

Duncanson, Laura, Huang, Wenli, Johnson, Kristofer, Swatantran, Anu, McRoberts, Ronald E., \& Dubayah, Ralph. 2017. Implications of allometric model selection for county-level biomass mapping. Carbon Balance and Management, 12(1), 18.

Goetz, Scott J., Steinberg, Daniel, Betts, Matthew G., Holmes, Richard T., Doran, Patrick J., Dubayah, Ralph, \& Hofton, Michelle. 2010. Lidar remote sensing variables predict breeding habitat of a Neotropical migrant bird. Ecology, 91(6), $1569-1576$.

Hackenberg, Jan, Wassenberg, Marc, Spiecker, Heinrich, \& Sun, Dongjing. 2015. Non destructive method for biomass prediction combining TLS derived tree volume and wood density. Forests, 6(4), 1274-1300.

Hagen, S., Harris, N., Saatchi, S.S., Pearson, T., Woodall, C.W., Ganguly, S., Domke, G.M., Braswell, B.H., Walters, B.F., Jenkins, J.C., Brown, S., Salas, W.A., Fore, A., Yu, Y., Nemani, R.R., Ipsan, C., \& Brown, K.R. 2016. CMS: Forest Carbon Stocks, Emissions, and Net Flux for the Conterminous US: 20052010. ORNL Distributed Active Archive Center.

Hancock, Steven, Anderson, Karen, Disney, Mathias, \& Gaston, Kevin J. 2017. Measurement of fine-spatial-resolution 3D vegetation structure with airborne waveform lidar: Calibration and validation with voxelised terrestrial lidar. Remote Sensing of Environment, 188(Jan.), 37-50.

Jin, Shengye, Tamura, Masayuki, \& Susaki, Junichi. 2016. A new approach to retrieve leaf normal distribution using terrestrial laser scanners. JOURNAL OF FORESTRY RESEARCH, 27(3), 631-638.

Liang, Xinlian, Kankare, Ville, Hyypp, Juha, Wang, Yunsheng, Kukko, Antero, Haggrn, Henrik, Yu, Xiaowei, Kaartinen, Harri, Jaakkola, Anttoni, Guan, Fengying, Holopainen, Markus, \& Vastaranta, Mikko. 2016. Terrestrial laser scanning in forest inventories. ISPRS Journal of Photogrammetry and Remote Sensing, Feb. 
McRoberts, Ronald E., Chen, Qi, Domke, Grant M., Sthl, Gran, Saarela, Svetlana, \& Westfall, James A. 2016. Hybrid estimators for mean aboveground carbon per unit area. Forest Ecology and Management, 378(Oct.), 44-56.

Mitchard, Edward T. A., Feldpausch, Ted R., Brienen, Roel J. W., LopezGonzalez, Gabriela, Monteagudo, Abel, Baker, Timothy R., Lewis, Simon L., Lloyd, Jon, Quesada, Carlos A., Gloor, Manuel, ter Steege, Hans, Meir, Patrick, Alvarez, Esteban, Araujo-Murakami, Alejandro, Aragao, Luiz E. O. C., Arroyo, Luzmila, Aymard, Gerardo, Banki, Olaf, Bonal, Damien, Brown, Sandra, Brown, Foster I., Ceron, Carlos E., Chama Moscoso, Victor, Chave, Jerome, Comiskey, James A., Cornejo, Fernando, Corrales Medina, Massiel, Da Costa, Lola, Costa, Flavia R. C., Di Fiore, Anthony, Domingues, Tomas F., Erwin, Terry L., Frederickson, Todd, Higuchi, Niro, Honorio Coronado, Euridice N., Killeen, Tim J., Laurance, William F., Levis, Carolina, Magnusson, William E., Marimon, Beatriz S., Marimon Junior, Ben Hur, Mendoza Polo, Irina, Mishra, Piyush, Nascimento, Marcelo T., Neill, David, Nunez Vargas, Mario P., Palacios, Walter A., Parada, Alexander, Pardo Molina, Guido, Pena-Claros, Marielos, Pitman, Nigel, Peres, Carlos A., Poorter, Lourens, Prieto, Adriana, RamirezAngulo, Hirma, Restrepo Correa, Zorayda, Roopsind, Anand, Roucoux, Katherine H., Rudas, Agustin, Salomao, Rafael P., Schietti, Juliana, Silveira, Marcos, de Souza, Priscila F., Steininger, Marc K., Stropp, Juliana, Terborgh, John, Thomas, Raquel, Toledo, Marisol, Torres-Lezama, Armando, van Andel, Tinde R., van der Heijden, Geertje M. F., Vieira, Ima C. G., Vieira, Simone, Vilanova-Torre, Emilio, Vos, Vincent A., Wang, Ophelia, Zartman, Charles E., Malhi, Yadvinder, \& Phillips, Oliver L. 2014. Markedly divergent estimates of Amazon forest carbon density from ground plots and satellites. Global Ecology and Biogeography, 23(8), 935-946.

Momo Takoudjou, Stphane, Ploton, Pierre, Sonk, Bonaventure, Hackenberg, Jan, Griffon, Sbastien, de Coligny, Francois, Kamdem, Narcisse Guy, Libalah, Moses, Mofack, Gislain II, Le Mogudec, Gilles, Plissier, Raphal, \& Barbier, Nicolas. 2017. Using Terrestrial Laser Scanning data to estimate large tropical trees biomass and calibrate allometric models: a comparison with traditional destructive approach. Methods in Ecology and Evolution.

Olagoke, Adewole, Proisy, Christophe, Fret, Jean-Baptiste, Blanchard, Elodie, Fromard, Franois, Mehlig, Ulf, de Menezes, Moirah Machado, dos Santos, Valdenira Ferreira, \& Berger, Uta. 2016. Extended biomass allometric equations for large mangrove trees from terrestrial LiDAR data. Trees, 30(3), 935-947.

Othmani, Ahlem, Piboule, Alexandre, Krebs, M., Stolz, C., \& Voon, LFC Lew Yan. 2011. Towards automated and operational forest inventories with T-Lidar. 
In: 11th International Conference on LiDAR Applications for Assessing Forest Ecosystems (SilviLaser 2011).

Raumonen, Pasi, Kaasalainen, Mikko, kerblom, Markku, Kaasalainen, Sanna, Kaartinen, Harri, Vastaranta, Mikko, Holopainen, Markus, Disney, Mathias, \& Lewis, Philip. 2013. Fast Automatic Precision Tree Models from Terrestrial Laser Scanner Data. Remote Sensing, 5(2), 491-520.

Saatchi, Sassan, Harris, Nancy L., Brown, Sandra, Lefsky, Michael, Mitchard, Edward T. A., Salas, William, Zutta, Brian R., Buermann, Wolfgang, Lewis, Simon L., Hagen, Stephen, Petrova, Silvia, White, Lee, Silman, Miles, \& Morel, Alexandra. 2011. Benchmark map of forest carbon stocks in tropical regions across three continents. Proceedings of the National Academy of Sciences of the United States of America, 108(24), 9899-9904.

Shugart, Herman H, Asner, Gregory P, Fischer, Rico, Huth, Andreas, Knapp, Nikolai, Le Toan, Thuy, \& Shuman, Jacquelyn K. 2015. Computer and remotesensing infrastructure to enhance large-scale testing of individual-based forest models. Frontiers in Ecology and the Environment, 13(9), 503-511.

Srinivasan, Shruthi, Popescu, Sorin C., Eriksson, Marian, Sheridan, Ryan D., \& $\mathrm{Ku}$, Nian-Wei. 2014. Multi-temporal terrestrial laser scanning for modeling tree biomass change. Forest Ecology and Management, 318(Apr.), 304-317.

Wilkes, Phil, Lau, Alvaro, Disney, Mathias, Calders, Kim, Burt, Andrew, Gonzalez de?Tanago, Jose, Bartholomeus, Harm, Brede, Benjamin, \& Herold, Martin. 2017. Data acquisition considerations for Terrestrial Laser Scanning of forest plots. Remote Sensing of Environment, 196(July), 140-153.

Yang, Xi, Tang, Jianwu, Mustard, John F., Lee, Jung-Eun, Rossini, Micol, Joiner, Joanna, Munger, J. William, Kornfeld, Ari, \& Richardson, Andrew D. 2015. Solar-induced chlorophyll fluorescence that correlates with canopy photosynthesis on diurnal and seasonal scales in a temperate deciduous forest: Fluorescence and photosynthesis. Geophysical Research Letters, 42(8), 2977-2987.

Yang, Xiaoyuan, Schaaf, Crystal, Strahler, Alan, Kunz, Thomas, Fuller, Nathan, Betke, Margrit, Wu, Zheng, Wang, Zhuosen, Theriault, Diane, \& Culvenor, Darius. 2013. Study of bat flight behavior by combining thermal image analysis with a LiDAR forest reconstruction. Canadian Journal of Remote Sensing, 39(s1), S112-S125. 
Supplementary Images

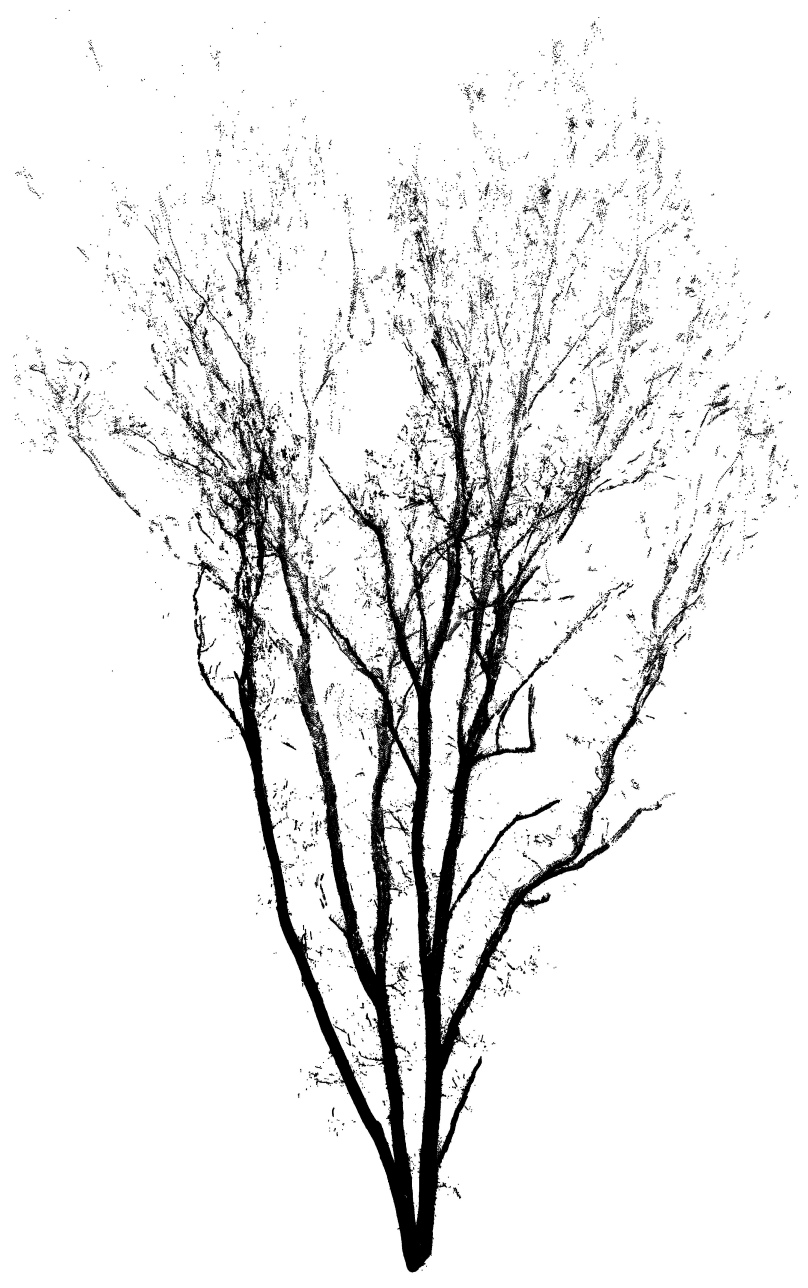




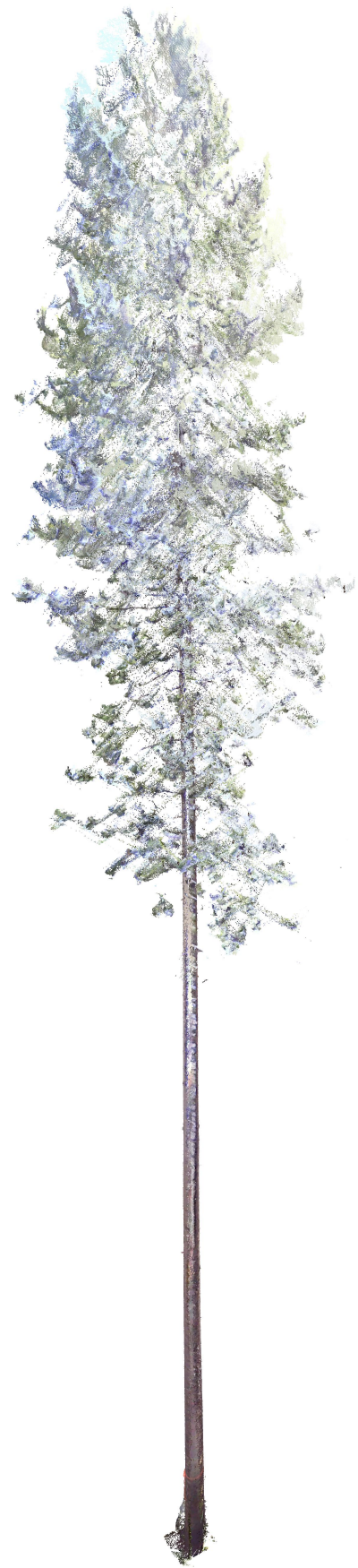

TLS scan of a lodgepole pine tree from Colorado State Forest selected for destructive sampling in Chapter 2. 


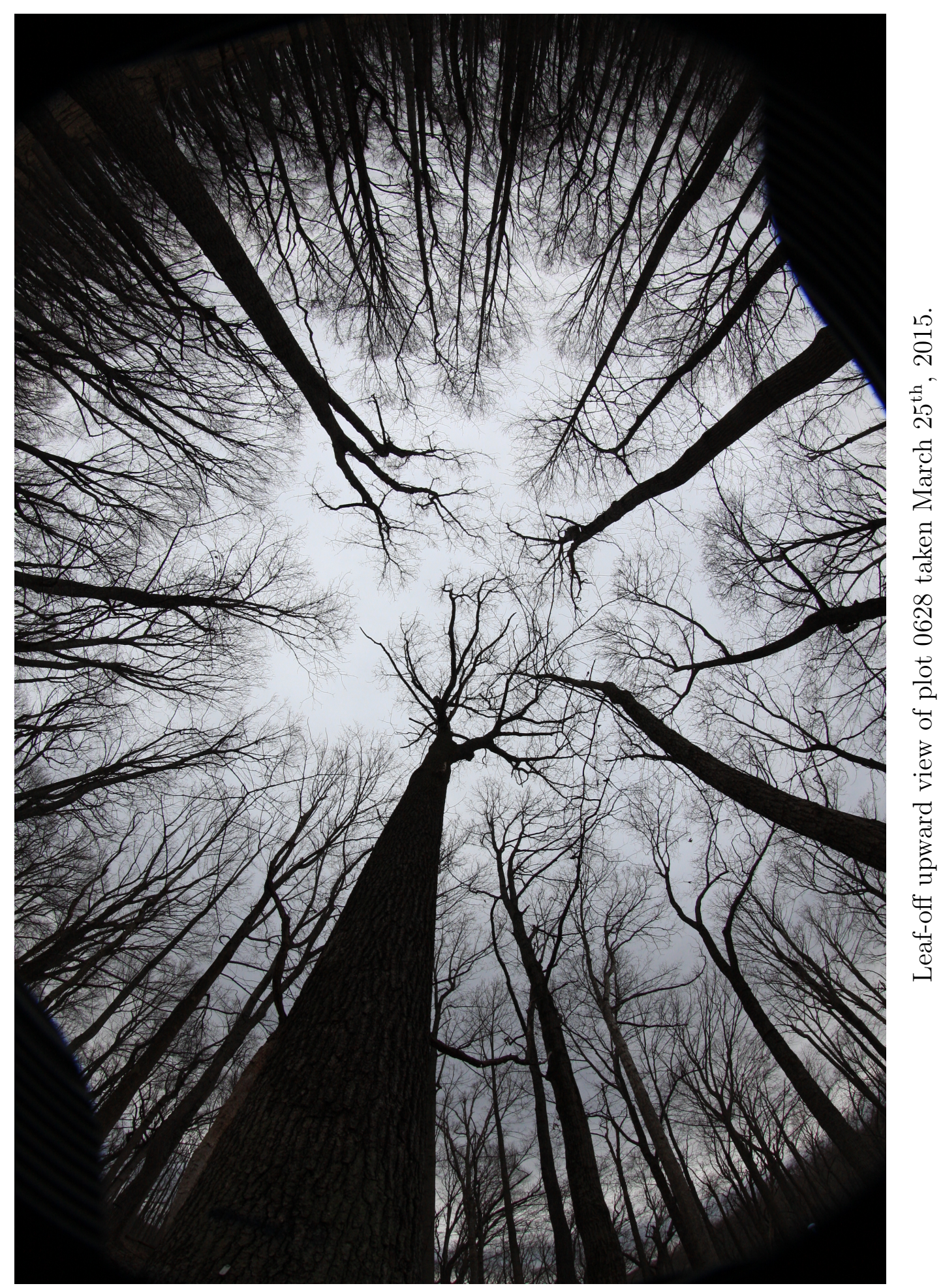




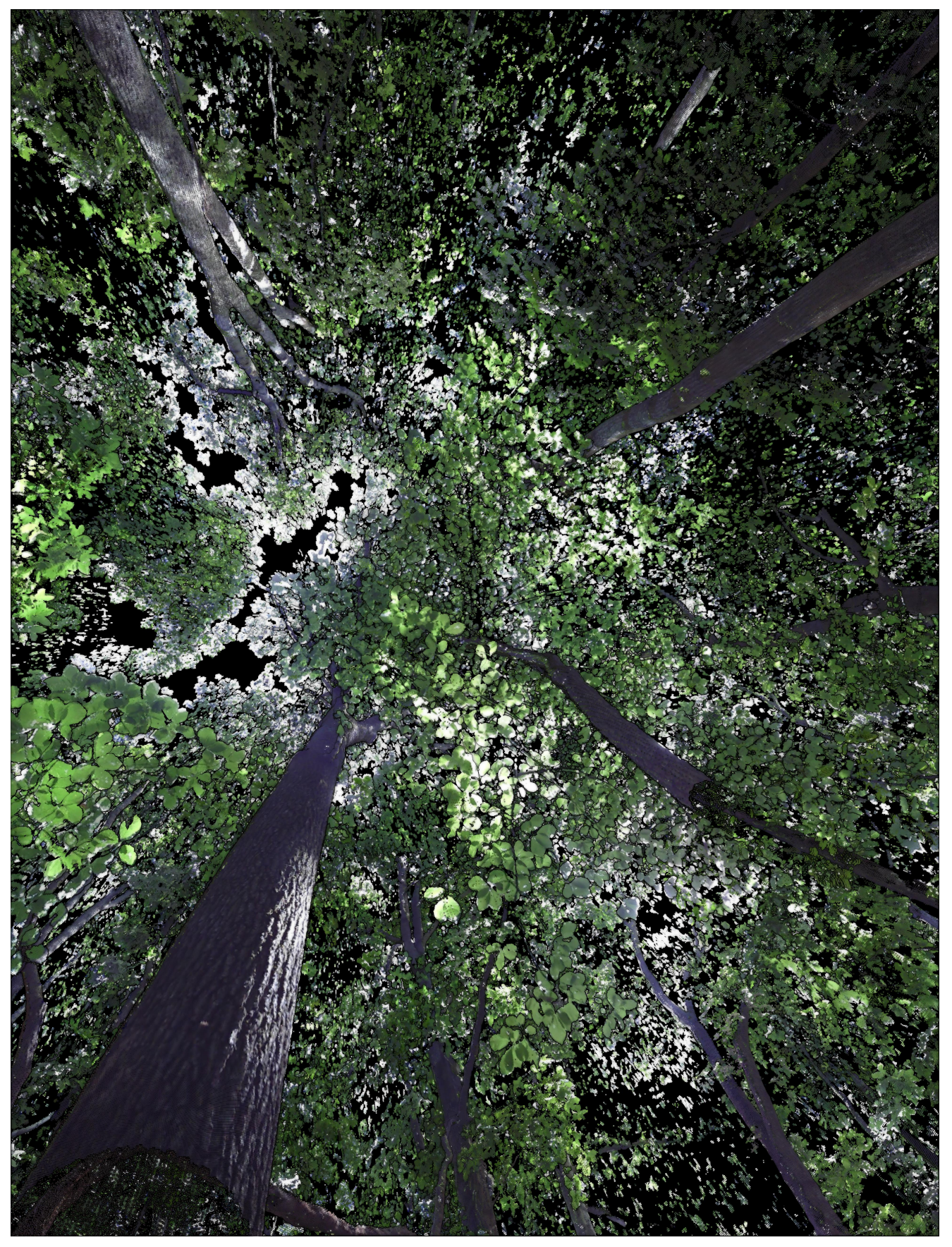

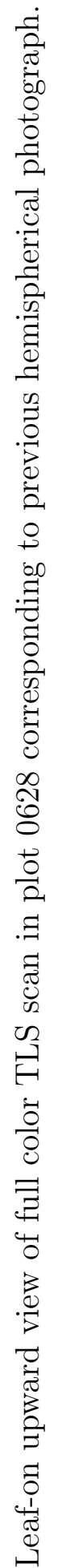




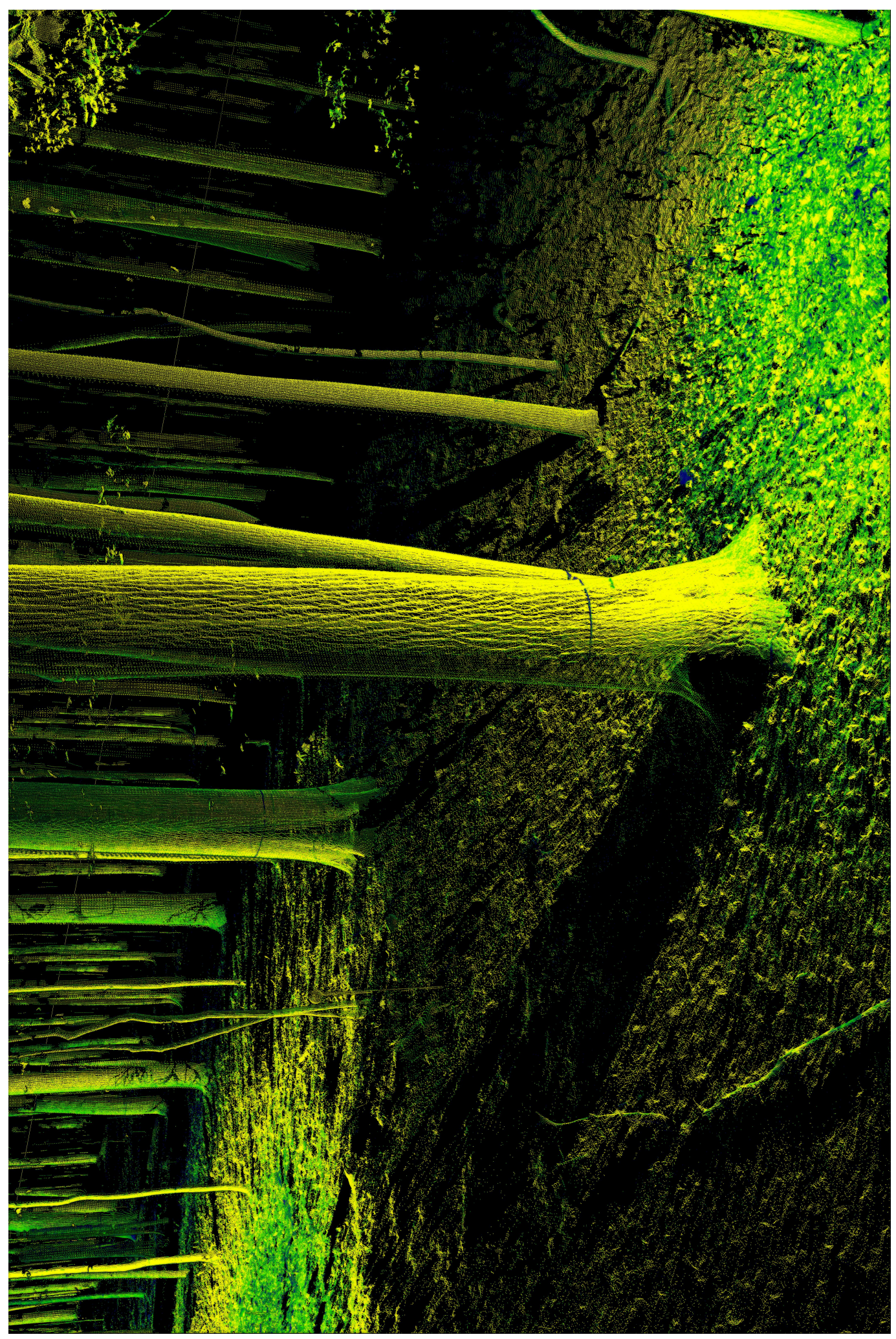

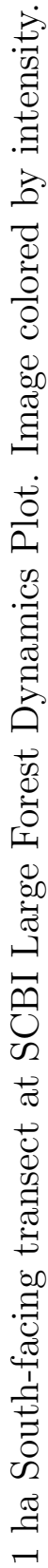




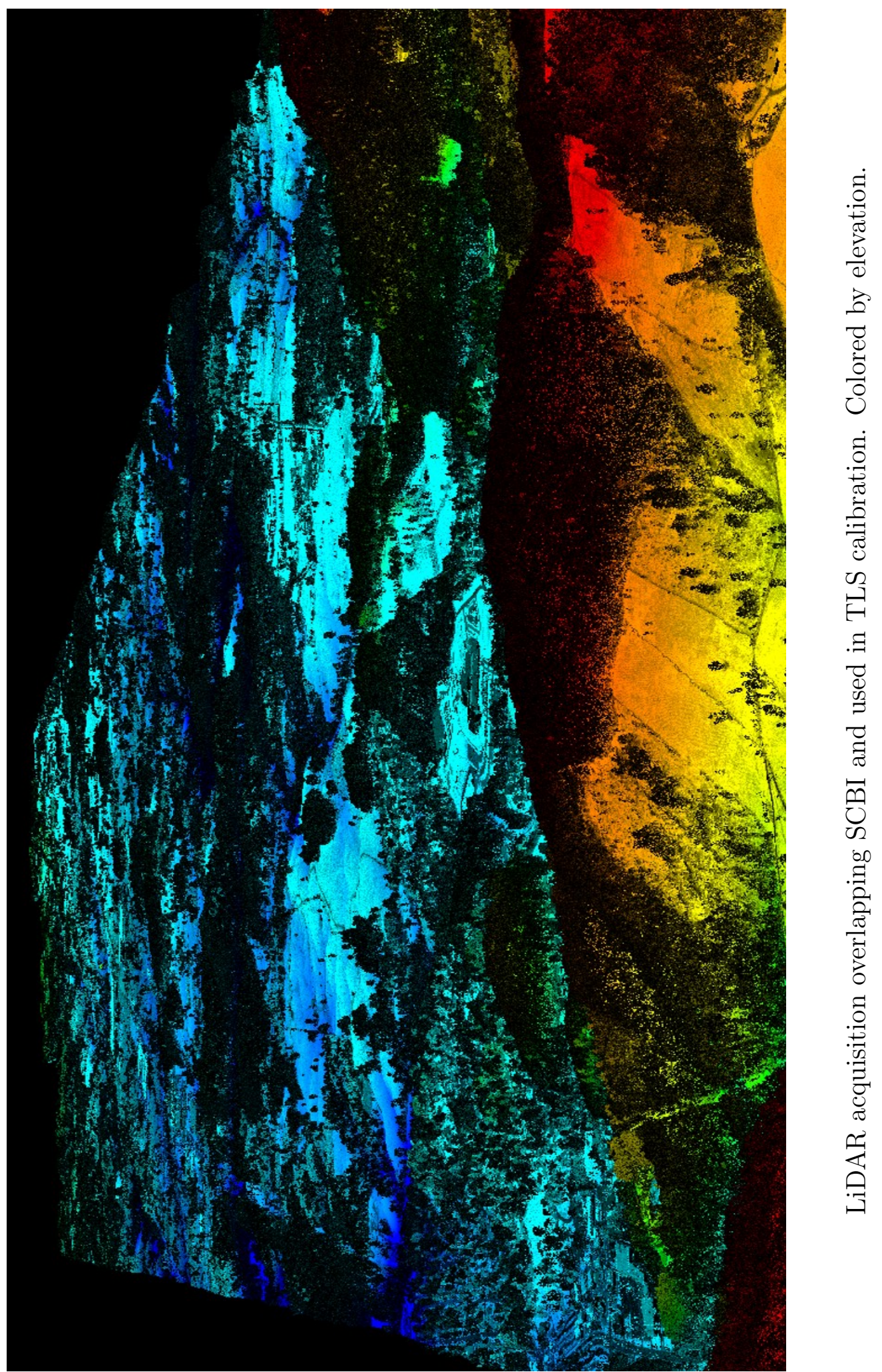


This dissertation was typeset using $\mathrm{AT}_{\mathrm{E}} \mathrm{X}$ 


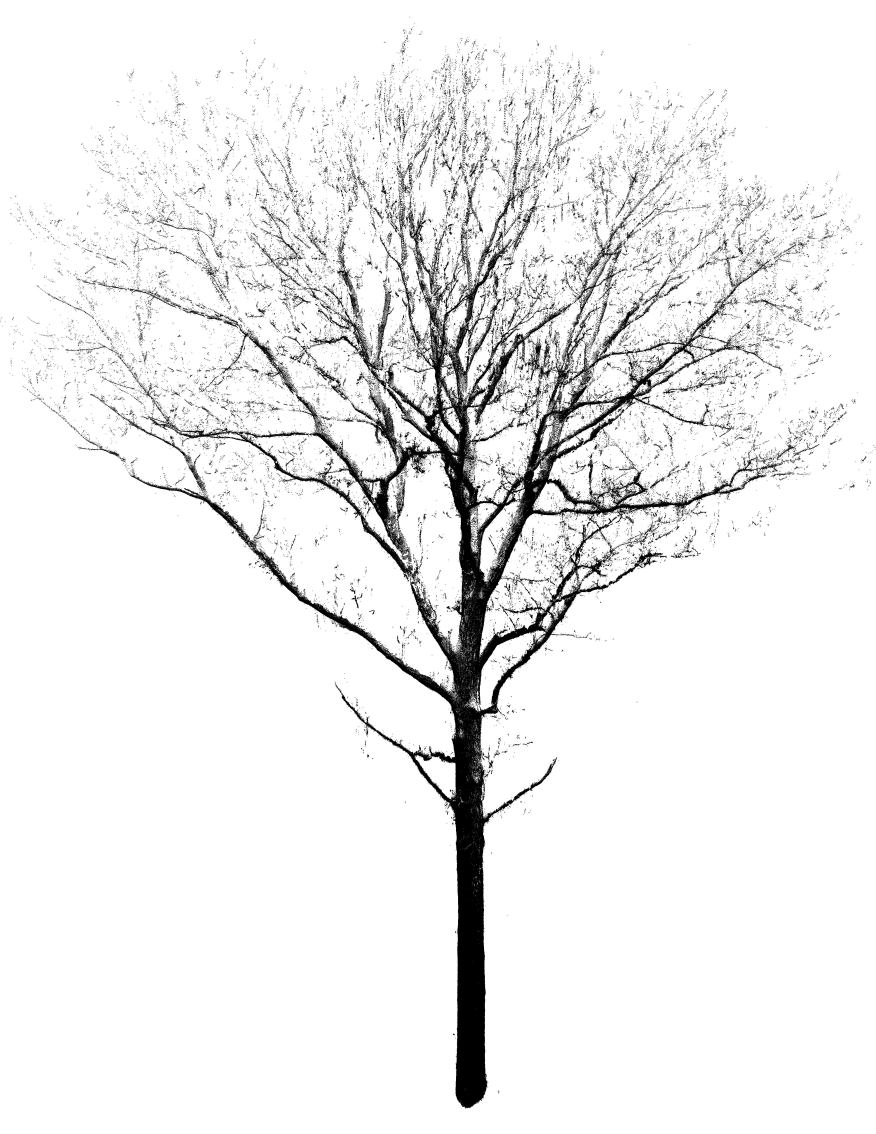

(c) Copyright by Atticus E. L. Stovall 2017

All Rights Reserved 